\title{
Sulfonylimidates as Nucleophiles in Catalytic Addition Reactions
}

\author{
Ryosuku Matsubara, Florian Berthiol, and Shū Kobayashi*
}

Department of Chemistry, School of Science, and Graduate School of Pharmaceutical Sciences, The University of Tokyo. The HFRE Division, ERATO, Japan Science Technology Agency (JST).

Hongo, Bunkyo-ku, Tokyo 113-0033, Japan.

\section{Supporting Information}

Experimental details and physical data of the products.

General. Melting points are uncorrected. ${ }^{1} \mathrm{H}$ and ${ }^{13} \mathrm{C}$ NMR spectra were recorded on a JEOL JNM-ECX-400, JNM-ECX-500 and JNM-ECX-600 spectrometer in $\mathrm{CDCl}_{3}$ or $\mathrm{C}_{6} \mathrm{D}_{6}$ unless otherwise noted. Tetramethylsilane (TMS) served as internal standard $(\delta=0)$ for ${ }^{1} \mathrm{H} \mathrm{NMR}$, and $\mathrm{CDCl}_{3}(\delta=77.0)$ and $\mathrm{C}_{6} \mathrm{D}_{6}(\delta=128.0)$ were used as internal standard for ${ }^{13} \mathrm{C}$ NMR. IR spectra were measured on a JASCO FT/IR-610 spectrometer. Column chromatography was conducted on Silica gel 60 (Merck) and preparative thin-layer chromatography was carried out using Wakogel B-5F. X-ray diffraction analysis was performed on a Rigaku-RAXIS-RAPID diffractometer. All reactions were carried out under argon atmosphere in dried glassware. All solvents were dried and distilled by standard procedures. N-alkoxycarbonyl imines $\mathbf{1 a}, \mathbf{1 b}$, $\mathbf{1 d - k}, \mathbf{1 0},{ }^{1} \mathrm{~N}$-sulfonylimines $\mathbf{1 c}, \mathbf{1 l - n}, \mathbf{1 p - q},{ }^{2}$ and $\mathrm{N}$-arylimino ester $\mathbf{1 r}^{3}$ were prepared according to the reported method.

\footnotetext{
${ }^{1}$ Kanazawa, A. M.; Denis, J.-N.; Greene, A. E. J. Am. Chem. Soc. 1994, 59, 1238.

${ }^{2}$ (a) Vishwakarma, L. C.; Stringer, O. D.; Davis, F. A. Org. Synth. 1987, 66, 203. (b) Love, B. E.; Raje, P. S.; Williams, T. C., II. Synlett 1994, 493. (c) Hodous, B. L.; Fu, G. C. J. Am. Chem. Soc. 2002, 124, 1578. and the references cited therein.

${ }^{3}$ Hagiwara, E.; Fujii, A.; Sodeoka, M. J. Am. Chem. Soc. 1998, 120, 2474. and the references cited therein.
} 
<smiles>CCOC(=O)/N=C/c1ccc(OC)cc1</smiles><smiles>[R]C=NC(=O)OCc1ccccc1</smiles>

1b: $\mathrm{R}=\mathrm{Ph}$

1d: $\mathrm{R}=p-\mathrm{MeOC}_{6} \mathrm{H}_{4}$

1e: $\mathrm{R}=p-\mathrm{FC}_{6} \mathrm{H}_{4}$

1f: $\mathrm{R}=m-\mathrm{MeC}_{6} \mathrm{H}_{4}$

1g: $\mathrm{R}=0-\mathrm{MeC}_{6} \mathrm{H}_{4}$

1h: $\mathrm{R}=m$-vinyl- $\mathrm{C}_{6} \mathrm{H}_{4}$

1i: $R=2$-furyl

1j: $R=2$-thienyl

1k: $R=2$-pyridyl

10: $\mathrm{R}=$ cyclopropyl
$\overbrace{}^{-T s}$

1c: $\mathrm{R}=\mathrm{Ph}$

1I: $\mathrm{R}=\mathrm{PhCH}=\mathrm{CH}$

$1 \mathrm{~m}: \mathrm{R}=\mathrm{tBu}$

1n: $R=$ cyclopropyl

1p: $\mathrm{R}=c-\mathrm{C}_{6} \mathrm{H}_{11}$

1q: $\mathrm{R}=\mathrm{Pr}$

Preparation of sulfonylimine $2{ }^{4}$ To a mixture of acetophenone $(3 \mathrm{ml}, 25.7 \mathrm{mmol})$, tosyl amide (4.403 g, $25.7 \mathrm{mmol}$ ) and DCE (dichloroethane, $40 \mathrm{ml}$ ) were added a solution of $\mathrm{TiCl}_{4}(4.879 \mathrm{~g}, 25.7 \mathrm{mmol})$ in DCE (10 ml) and $\mathrm{Et}_{3} \mathrm{~N}(7.89 \mathrm{ml}, 56.6$ mmol), successively at $0{ }^{\circ} \mathrm{C}$. The mixture was stirred at room temperature (RT) for $18 \mathrm{~h}$, and poured into saturated $\mathrm{NaHCO}_{3}$ aqueous solution at $0{ }^{\circ} \mathrm{C}$. After filtration, the filtrate was extracted by $\mathrm{CH}_{2} \mathrm{Cl}_{2}$ twice, and the organics were dried over anhydrous $\mathrm{Na}_{2} \mathrm{SO}_{4}$. Filtration and evaporation of the solvents afforded the crude product, which was purified by column chromatography on neutral $\mathrm{SiO}_{2}$ and recrystallization (hexane/AcOEt) to give us sulfonylimine 2 ( $1 \mathrm{~g}, 15 \%)$. The reaction condition has not been optimized.

$\begin{array}{ll}N_{\mathrm{Me}}{ }_{\mathrm{Ph}} & \text { 1-phenyl- } \mathrm{N} \text {-tosylethanimine (2): } \mathrm{Mp} .89-90{ }^{\circ} \mathrm{C} ;{ }^{1} \mathrm{H} \mathrm{NMR}\left(\mathrm{CDCl}_{3}\right) \delta= \\ & 7.86-95(\mathrm{~m}, 4 \mathrm{H}), 7.47-7.54(\mathrm{~m}, 1 \mathrm{H}), 7.37-7.43(\mathrm{~m}, 2 \mathrm{H}), 7.33(\mathrm{~d}, 2 \mathrm{H}, J\end{array}$ $=7.9 \mathrm{~Hz}), 2.98(\mathrm{~s}, 3 \mathrm{H}), 2.43(\mathrm{~s}, 3 \mathrm{H}) ;{ }^{13} \mathrm{C} \mathrm{NMR}\left(\mathrm{CDCl}_{3}\right) \delta=179.7,143.5,138.7$, 137.5, 133.1, 129.4, 128.6, 128.2, 127.0, 21.5, 21.1; IR (neat) 1698, 1600, 1590, 1570, 1448, 1373, 1308, 1286, 1181, 1153, 1083, 1017, 984, 819, 806, 768, 724, 687, 611, $582,546,531 \mathrm{~cm}^{-1}$; HRMS (FAB); Exact mass calcd for $\mathrm{C}_{15} \mathrm{H}_{16} \mathrm{NO}_{2} \mathrm{~S}[\mathrm{M}+\mathrm{H}]^{+}$, 274.0901. Found 274.0899.

Preparation of sulfonylimidates. Sulfonylimidates 6a-6h were synthesized by using the similar method to the reported one. ${ }^{5}$ General procedure is as follows (eqations 4 and 5).

Imidate $\mathrm{HCl}$ salt formation.(eq. 4) $\mathrm{HCl}$ gas was bubbled into a mixture of nitrile (400 mmol) and alcohol (400 mmol) for 10-20 min (exothermic). In some cases

\footnotetext{
${ }^{4}$ Matsubara, R.; Doko, T.; Uetake, R.; Kobayashi, S. Angew. Chem. Int. Ed. 2007, 46, 3047.

${ }^{5}$ Kupfer, R.; Nagel, M.; Würthwein, E.-U.; Allmann, R. Chem. Ber. 1985, 118, 3089.
} 
(especially when $\mathrm{iPrOH}$ was used as an alcohol), temperature higher than room temperature was necessary for the reaction, that is why the reaction was not cooled during the exothermic reaction. After completion of bubbling of $\mathrm{HCl}$, the mixture was left for 3-10 h under Ar atmosphere. Removal of all the volatiles by evaporation gave us almost pure imidate $\mathrm{HCl}$ salt in $40-80 \%$ yield (moderate yield may be caused by evaporation of the volatile starting materials during the course of the exothermic reactions), which can be used in the next reaction without further purification. Further purification is possible by washing the solid with dry $\mathrm{Et}_{2} \mathrm{O}$. Imidate $\mathrm{HCl}$ salts are hygroscopic, but can be kept under inert gas atmosphere in the refrigerator for at least 1 year.

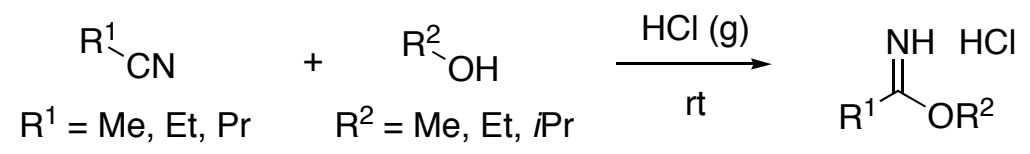

Representative procedure for the syntheses of sulfonylimidates. (eq. 5) To a solution of imidate $\mathrm{HCl}$ salt $\mathbf{A}(3.01 \mathrm{~g}, 19.85 \mathrm{mmol})$ in dichloromethane (DCM, 50 $\mathrm{mL})$ was added $\mathrm{Et}_{3} \mathrm{~N}(8.3 \mathrm{~mL}, 59.55 \mathrm{mmol})$ dropwise at $\mathrm{rt}$. To the resultant suspension was added $\mathrm{TsCl}(3.785 \mathrm{~g}, 19.85 \mathrm{mmol})$ and DMAP (242.5 mg, 1.985 mmol). The reaction mixture was stirred until $\mathrm{TsCl}$ was consumed $(40 \mathrm{~h})$. The mixture was poured into water, and extracted with DCM. The organics were dried over anhydrous $\mathrm{Na}_{2} \mathrm{SO}_{4}$. Filtration and evaporation of the solvents afforded the crude product, which was purified by column chromatography on $\mathrm{SiO}_{2}$ to give us a pure sulfonylimidate $\mathbf{6 g}$ ( $4.565 \mathrm{~g}, 85 \%$ yield).
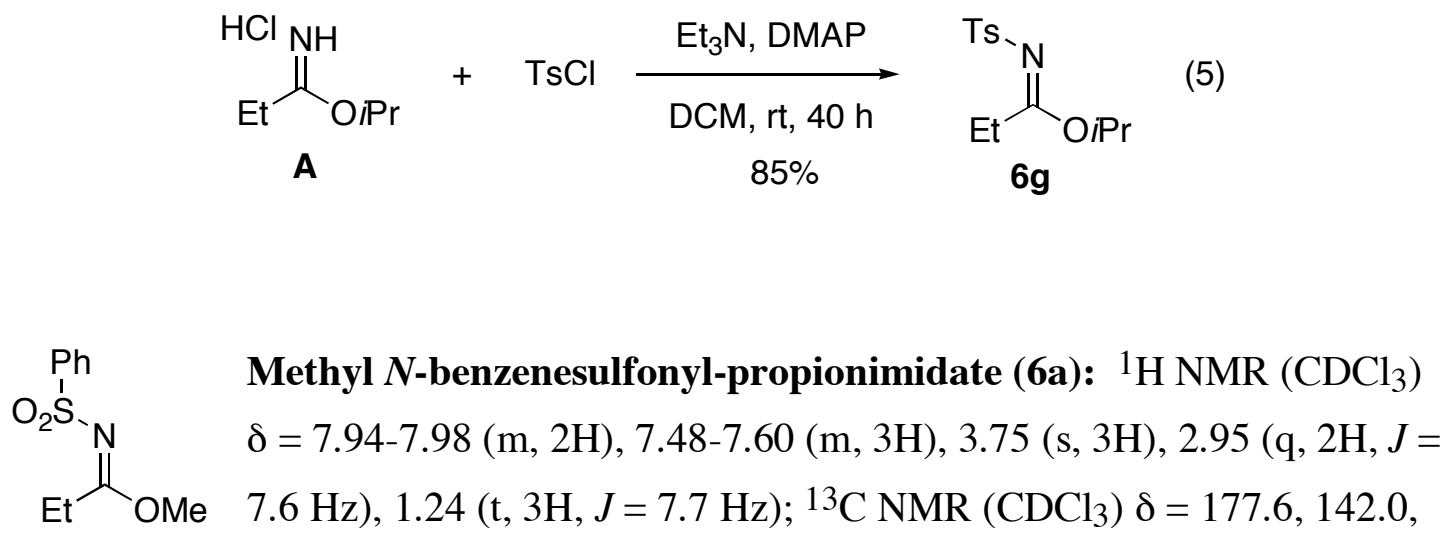
132.4, 128.7, 126.5, 55.4, 27.5, 10.0; IR (neat) 3063, 2985, 2950, 2887, 1602, 1541, 1507, 1446, 1377, 1305, 1235, 1155, 1091, 1023, 945, 798, 758, 733, 690, 621, 585, $536 \mathrm{~cm}^{-1}$; HRMS (FAB); Exact mass calcd for $\mathrm{C}_{10} \mathrm{H}_{14} \mathrm{NO}_{3} \mathrm{~S}[\mathrm{M}+\mathrm{H}]^{+}, 228.0694$. Found 228.0697. 


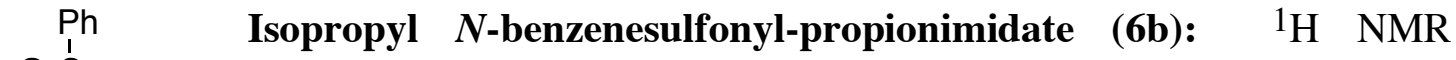
$\mathrm{O}_{2} \mathrm{~S}_{\mathrm{N}} \quad\left(\mathrm{CDCl}_{3}\right) ; \delta=8.06-8.09(\mathrm{~m}, 2 \mathrm{H}), 7.60-7.64(\mathrm{~m}, 3 \mathrm{H}), 4.70$ (sept, $1 \mathrm{H}, J=$ Et $\left.\mathrm{O}_{\mathrm{Pr}} 6.3 \mathrm{~Hz}\right), 2.88(\mathrm{qd}, 2 \mathrm{H}, J=7.3,1.7 \mathrm{~Hz}), 1.05(\mathrm{td}, 3 \mathrm{H}, J=7.3,1.2 \mathrm{~Hz})$, $0.83(\mathrm{~d}, 6 \mathrm{H}, J=6.3 \mathrm{~Hz}) ;{ }^{13} \mathrm{C} \mathrm{NMR}\left(\mathrm{CDCl}_{3}\right) \delta=176.0,143.6,132.0,128.8,126.9$, 71.6, 28.2, 20.9, 10.4; IR (neat) 3055, 2988, 1506, 1448, 1308, 1265, 1157, 1093, 896, 740, 705, 634, 459, 445, $413 \mathrm{~cm}^{-1}$; HRMS (FAB); Exact mass calcd for $\mathrm{C}_{12} \mathrm{H}_{18} \mathrm{NO}_{3} \mathrm{~S}$ $[\mathrm{M}+\mathrm{H}]^{+}, 256.1007$. Found 256.1010.

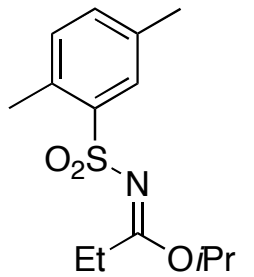

Isopropyl $N$-(2,5-xylylsulfonyl)-propionimidate (6c): Mp. 32$33{ }^{\circ} \mathrm{C} ;{ }^{1} \mathrm{H} \mathrm{NMR}\left(\mathrm{CDCl}_{3}\right) ; \delta=8.20(\mathrm{~s}, 1 \mathrm{H}), 6.90(\mathrm{~d}, 1 \mathrm{H}, J=7.4 \mathrm{~Hz})$, $6.84(\mathrm{dd}, 1 \mathrm{H}, J=7.4,1.4 \mathrm{~Hz}), 4.74(\mathrm{sept}, 1 \mathrm{H}, J=6.3 \mathrm{~Hz}), 2.91(\mathrm{q}$, $2 \mathrm{H}, J=7.4 \mathrm{~Hz}), 2.77(\mathrm{~s}, 3 \mathrm{H}), 1.94(\mathrm{~s}, 3 \mathrm{H}), 1.07(\mathrm{t}, 3 \mathrm{H}, J=7.4 \mathrm{~Hz})$, $0.83(\mathrm{~d}, 6 \mathrm{H}, J=6.3 \mathrm{~Hz}) ;{ }^{13} \mathrm{C} \mathrm{NMR}\left(\mathrm{CDCl}_{3}\right) \delta=176.2,141.2,135.9$, $134.4,133.0,132.2,128.9,71.2,28.4,21.1,20.6,20.2,10.4$; IR (neat) 3055, 2988, 1590, 1458, 1308, 1265, 1154, 1092, 1066, 896, 740, 705, 642, 459, $413 \mathrm{~cm}^{-1}$; HRMS (ESI); Exact mass calcd for $\mathrm{C}_{14} \mathrm{H}_{22} \mathrm{NO}_{3} \mathrm{~S}[\mathrm{M}+\mathrm{H}]^{+}, 284.1320$. Found 284.1323.

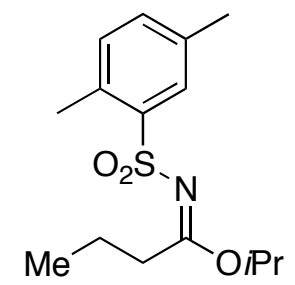

Isopropyl $N$-(2,5-xylylsulfonyl)-butyrimidate (6d): $\quad$ Mp. 35$36{ }^{\circ} \mathrm{C} ;{ }^{1} \mathrm{H} \mathrm{NMR}\left(\mathrm{CDCl}_{3}\right) ; \delta=8.18(\mathrm{~s}, 1 \mathrm{H}), 6.92(\mathrm{~d}, 1 \mathrm{H}, J=8.0 \mathrm{~Hz})$, $6.84(\mathrm{~d}, 1 \mathrm{H}, J=8.0 \mathrm{~Hz}), 4.77$ (sept, $1 \mathrm{H}, J=6.2 \mathrm{~Hz}), 2.87-2.93(\mathrm{~m}$, 2H), 2.77 (s, 3H), 1.95 (s, 3H), $1.65(\mathrm{sext}, 2 \mathrm{H}, J=7.4 \mathrm{~Hz}), 0.87$ (d, $6 \mathrm{H}, J=6.2 \mathrm{~Hz}), 0.82(\mathrm{t}, 3 \mathrm{H}, J=7.4 \mathrm{~Hz}) ;{ }^{13} \mathrm{C} \mathrm{NMR}\left(\mathrm{CDCl}_{3}\right) \delta=$ $175.3,141.2$, 135.9, 134.4, 133.0, 132.2, 128.3, 71.2, 36.5, 21.1, 20.6, 20.2, 20.0, 13.8; IR (neat) 3055, 2987, 2933, 2878, 1590, 1457, 1373, 1303, 1219, 1154, 1102 , 1067, 918, 896, 739, 706, 641, 459, $412 \mathrm{~cm}^{-1}$; HRMS (FAB); Exact mass calcd for $\mathrm{C}_{15} \mathrm{H}_{24} \mathrm{NO}_{3} \mathrm{~S}[\mathrm{M}+\mathrm{H}]^{+}, 298.1477$. Found 298.1465.

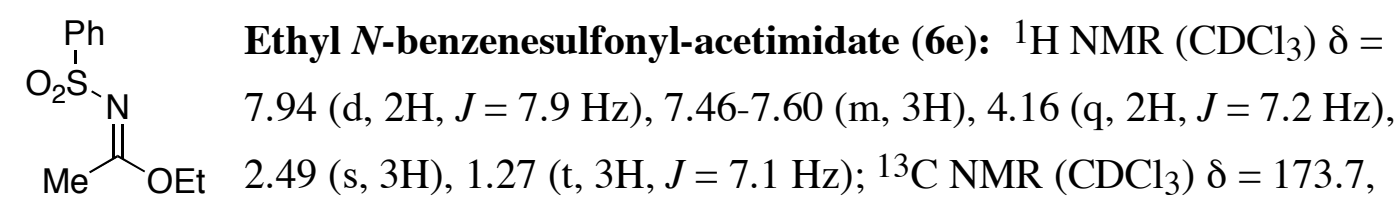
141.7, 132.3, 128.6, 126.5, 64.6, 20.5, 13.5; IR (neat) 3066, 2986, 2940, 2904, 1748, 1606, 1474, 1447, 1396, 1376, 1306, 1158, 1118, 1093, 1046, 1121, 998, 869, 803, $735,689,633,588,567,525 \mathrm{~cm}^{-1}$; HRMS (FAB); Exact mass calcd for $\mathrm{C}_{10} \mathrm{H}_{14} \mathrm{NO}_{3} \mathrm{~S}$ $[\mathrm{M}+\mathrm{H}]^{+}, 228.0694$. Found 228.0695.

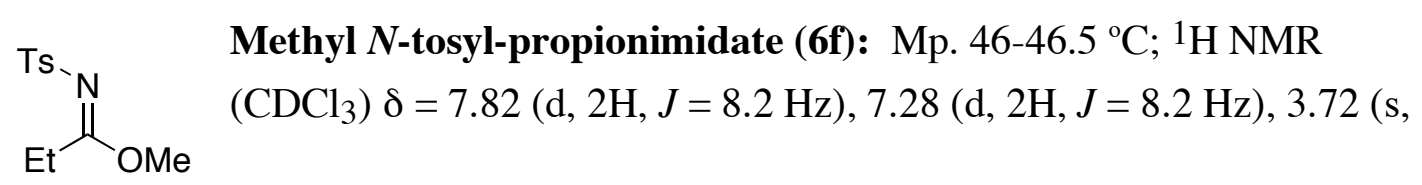


3H), $2.92(\mathrm{q}, 2 \mathrm{H}, J=7.6 \mathrm{~Hz}), 2.40(\mathrm{~s}, 3 \mathrm{H}), 1.22(\mathrm{t}, 3 \mathrm{H}, J=7.6 \mathrm{~Hz}) ;{ }^{13} \mathrm{C} \mathrm{NMR}$ $\left(\mathrm{CDCl}_{3}\right) \delta=177.4,143.1,139.1,129.3,126.6,55.4,27.4,21.5,10.0$; IR (neat) 2983, 2949, 1605, 1496, 1464, 1442, 1378, 1314, 1235, 1190, 1155 1092, 1030, 1018, 944.9, 815, 709, 687, 593, $555 \mathrm{~cm}^{-1}$; HRMS (FAB); Exact mass calcd for $\mathrm{C}_{11} \mathrm{H}_{16} \mathrm{NO}_{3} \mathrm{~S}$ $[\mathrm{M}+\mathrm{H}]^{+}, 242.0851$. Found 242.0856.

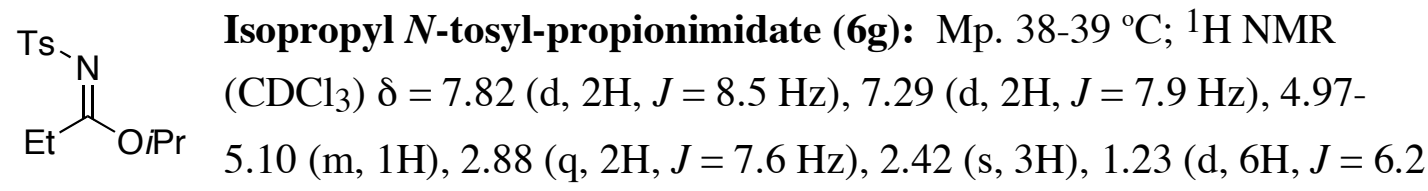
$\mathrm{Hz}), 1.21(\mathrm{t}, 3 \mathrm{H}, J=6.2 \mathrm{~Hz}) ;{ }^{13} \mathrm{C} \mathrm{NMR}\left(\mathrm{CDCl}_{3}\right) \delta=176.3,142.9,139.4,129.3$, 126.4, 71.9, 27.8, 21.5, 21.1, 10.1; IR (neat) 2983, 2942, 1597, 1496, 1465, 1383, 1356, 1312, 1235, 1183, 1157, 1093, 1032, 1017, 955, 909, 838, 814, 799, 692, 600, 555, $529 \mathrm{~cm}^{-1}$; HRMS (FAB); Exact mass calcd for $\mathrm{C}_{13} \mathrm{H}_{20} \mathrm{NO}_{3} \mathrm{~S}[\mathrm{M}+\mathrm{H}]^{+}, 270.1164$. Found 270.1167.<smiles>CCC(=NSc1cc(C)ccc1C)OC</smiles>

Methyl $N$-(2,5-xylylsulfonyl)-propionimidate (6h): Mp. $71-72{ }^{\circ} \mathrm{C}$; ${ }^{1} \mathrm{H}$ NMR $\left(\mathrm{C}_{6} \mathrm{D}_{6}\right) \delta=8.15-8.17(\mathrm{~m}, 1 \mathrm{H}), 7.16-7.17(\mathrm{~m}, 1 \mathrm{H}), 6.85-$ $6.94(\mathrm{~m}, 2 \mathrm{H}), 3.10(\mathrm{~s}, 3 \mathrm{H}), 2.87(\mathrm{q}, 2 \mathrm{H}, J=7.6 \mathrm{~Hz}), 2.72(\mathrm{~s}, 3 \mathrm{H})$, $1.95(\mathrm{~s}, 3 \mathrm{H}), 1.02(\mathrm{t}, 3 \mathrm{H}, J=7.4 \mathrm{~Hz}) ;{ }^{13} \mathrm{C} \mathrm{NMR}\left(\mathrm{C}_{6} \mathrm{D}_{6}\right) \delta=177.1$, $140.9,135.9,134.5,133.1,132.3,128.9,54.6,27.9,20.5,20.2,10.0$; IR (neat) 2982, 2949, 2884, 1602, 1491, 1461, 1442, 1378, 1307, 1234, 1191, 1154 , 1142, 1094, 1065, 1028, 944, 884, 821, 798, 747, 705, 630, 579, 548, $503 \mathrm{~cm}^{-1}$; HRMS (FAB); Exact mass calcd for $\mathrm{C}_{12} \mathrm{H}_{18} \mathrm{NO}_{3} \mathrm{~S}[\mathrm{M}+\mathrm{H}]^{+}, 256.1007$. Found 256.1000 .

General procedure of addition reactions of sulfonylimidates to imines. To MS 4A $(50 \mathrm{mg})$ was added a solution of imine $(0.45 \mathrm{mmol})$ in $\mathrm{DMF}(0.5 \mathrm{ml})$ and sulfonylimidate $(0.3 \mathrm{mmol})$. The mixture was cooled to $0{ }^{\circ} \mathrm{C}$ and a solution of DBU (1,8-diazabicyclo[5.4.0]undec-7-ene, $5 \mathrm{~mol} \%)$ in DMF (100 $\mu \mathrm{l})$ was added. The mixture was stirred for $24 \mathrm{~h}$ and then diluted by addition of $\mathrm{Et}_{2} \mathrm{O}$. The mixture obtained after filtration (for removal of MS 4A) was washed with water 3 times, then dried over anhydrous $\mathrm{Na}_{2} \mathrm{SO}_{4}$. Filtration and removal of solvents afforded the crude product. Diastereo ratio was determined by ${ }^{1} \mathrm{H}$ NMR spectroscopy analysis of the crude product. Purification of the crude product was conducted by chromatography on $\mathrm{SiO}_{2}$, to afford the desired product 7. When it was difficult to determine the 
deastereo ratio at the crude product stage, the ratio was determined by the weight of the separated diastereomers after purification.

Procedure of addition reaction of sulfonylimidate 6e to $\mathrm{N}$-Boc imine 1a (Table 2, entry 3). To MS 4A (50 mg) was added a solution of imine 1a (53.2 mg, $0.3 \mathrm{mmol})$ in DMF $(0.5 \mathrm{ml})$. The mixture was cooled to $0{ }^{\circ} \mathrm{C}$, then sulfonylimidate $6 \mathrm{e}(340.9 \mathrm{mg}$, $1.5 \mathrm{mmol})$ and a solution of DBU in DMF (10 mol\%, 100 $\mu \mathrm{l})$ were added successively. The mixture was stirred for $30 \mathrm{~min}$ at $0{ }^{\circ} \mathrm{C}$, and then diluted by addition of $\mathrm{Et}_{2} \mathrm{O}$. The mixture obtained after filtration (for removal of MS 4A) was washed with water 3 times, then dried over anhydrous $\mathrm{Na}_{2} \mathrm{SO}_{4}$. Filtration and removal of solvents afforded the crude product. Purification of the crude product was conducted by chromatography on $\mathrm{SiO}_{2}$, to afford the desired product $7 \mathbf{f}(95.8 \mathrm{mg}, 79 \%)$ and the recovered sulfonylimidate $\mathbf{6 e}(275 \mathrm{mg}, 1.21 \mathrm{mmol})$.

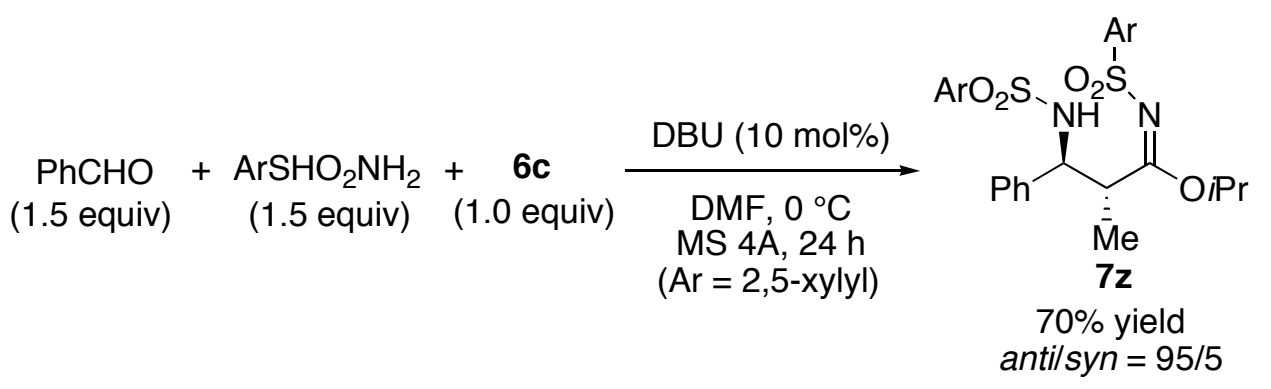

Procedure of 3 component reaction between benzaldehyde, sulfonamide, and sulfonylimidate 6c (equation 6). To a suspension of MS 4A (100 mg) in DMF (0.5 $\mathrm{ml})$ were added benzaldehyde $(45.7 \mu \mathrm{l}, 0.45 \mathrm{mmol})$ and 2,5-xylylsulfonylamide $(83.4$ $\mathrm{mg}, 0.45 \mathrm{mmol}$ ). The mixture was stirred at RT for $20 \mathrm{~min}$, and then cooled to $0{ }^{\circ} \mathrm{C}$. To the suspension were added sulfonylimidate $\mathbf{6 c}(85.0 \mathrm{mg}, 0.3 \mathrm{mmol})$ and a solution of DBU in DMF (10 mol\%, $100 \mu \mathrm{l})$ successively. The mixture was stirred for $23 \mathrm{~h}$ at $0{ }^{\circ} \mathrm{C}$, and then diluted by addition of $\mathrm{Et}_{2} \mathrm{O}$. The mixture obtained after filtration (for removal of MS 4A) was washed with water 3 times, then dried over anhydrous $\mathrm{Na}_{2} \mathrm{SO}_{4}$. Filtration and removal of solvents afforded the crude product. Diastereo ratio was determined by ${ }^{1} \mathrm{H}$ NMR spectroscopy analysis of the crude product (anti/syn $=95 / 5$ ). Purification of the crude product was conducted by chromatography on $\mathrm{SiO}_{2}$, to afford the desired product $\mathbf{7 z}(116.8 \mathrm{mg}, 70 \%$ yield).<smiles>CCOC(=O)N[C@H](c1ccccc1)C(C)/C(=N\Sc1ccccc1)OC</smiles>

Methyl anti- $N$-(benzenesulfonyl)-3-(ethoxycarbonylamino)2-methyl-3-phenylpropionimidate (anti-7a): ${ }^{1} \mathrm{H}$ NMR $\left(\mathrm{C}_{6} \mathrm{D}_{6}\right) \delta=8.07-8.10(\mathrm{~m}, 2 \mathrm{H}), 7.38(\mathrm{~d}, 2 \mathrm{H}, J=7.6 \mathrm{~Hz}), 7.04-$ $7.08(\mathrm{~m}, 2 \mathrm{H}), 6.95-7.03(\mathrm{~m}, 4 \mathrm{H}), 6.74(\mathrm{~d}, 1 \mathrm{H}, J=9.6 \mathrm{~Hz}), 5.08$ $(\mathrm{t}, 1 \mathrm{H}, J=10.3), 4.38-4.45(\mathrm{~m}, 1 \mathrm{H}), 3.88-3.98(\mathrm{~m}, 2 \mathrm{H}), 3.20(\mathrm{~s}, 3 \mathrm{H}), 0.92(\mathrm{t}, 3 \mathrm{H}, J=$ 
$6.9 \mathrm{~Hz}), 0.88(\mathrm{t}, 3 \mathrm{H}, J=7.2 \mathrm{~Hz}) ;{ }^{13} \mathrm{C} \mathrm{NMR}\left(\mathrm{C}_{6} \mathrm{D}_{6}\right) \delta=14.6,14.9,45.6,55.2,59.4$, $60.8,127.0,127.6,128.8,129.1,132.4,140.9,142.6,155.8,177.9$; IR (neat) 3375, 3064, 3030, 2982, 2951, 1722, 1602, 1518, 1447, 1369, 1303, 1245, 1222, 1152, 1090, 1059, 1025, 999, 950, 907, 842, 772, 758, 734, 701, 689, 623, 525, $426 \mathrm{~cm}^{-1}$; HRMS (FAB); Exact mass calcd for $\mathrm{C}_{20} \mathrm{H}_{25} \mathrm{~N}_{2} \mathrm{O}_{5} \mathrm{~S}[\mathrm{M}+\mathrm{H}]^{+}, 405.1484$. Found 405.1494.<smiles>CCOC(=O)N[C@H](c1ccccc1)C(C)/C(=N\Sc1ccccc1)OC</smiles>

Methyl syn- $N$-(benzenesulfonyl)-3-(ethoxycarbonylamino)2-methyl-3-phenylpropionimidate (syn-7a): Mp. $130-131{ }^{\circ} \mathrm{C}$; ${ }^{1} \mathrm{H}$ NMR $\left(\mathrm{C}_{6} \mathrm{D}_{6}\right) \delta=7.93-7.96(\mathrm{~m}, 2 \mathrm{H}), 7.48(\mathrm{~d}, 2 \mathrm{H}, J=6.9$ $\mathrm{Hz}), 7.13-7.18(\mathrm{~m}, 3 \mathrm{H}), 7.02(\mathrm{t}, 1 \mathrm{H}, J=7.2 \mathrm{~Hz}), 6.90-6.95(\mathrm{~m}$, $3 \mathrm{H}), 5.38(\mathrm{t}, 1 \mathrm{H}, J=10 \mathrm{~Hz}), 4.76(\mathrm{~d}, 1 \mathrm{H}, J=9.6 \mathrm{~Hz}), 4.30-4.39(\mathrm{~m}, 1 \mathrm{H}), 3.88-4.06$ $(\mathrm{m}, 2 \mathrm{H}), 2.88(\mathrm{~s}, 3 \mathrm{H}), 1.47(\mathrm{~d}, 3 \mathrm{H}, J=6.9 \mathrm{~Hz}), 0.94(\mathrm{t}, 3 \mathrm{H}, J=6.9 \mathrm{~Hz}) ;{ }^{13} \mathrm{C} \mathrm{NMR}$ $\left(\mathrm{C}_{6} \mathrm{D}_{6}\right) \delta=176.4,156.3,143.0,141.2,132.1,128.8,128.7,126.9,60.8,60.1,57.3$, 54.6, 44.5, 20.5, 15.5, 14.6, 14.2; IR (neat) 3343, 3063, 2924, 2853, 1715, 1697, 1607, 1532, 1448, 1375, 1305, 1287, 1261, 1237, 1155, 1084, 1035, 951, 927, 905, 733, 701, 689, 622, $586 \mathrm{~cm}^{-1}$; HRMS (FAB); Exact mass calcd for $\mathrm{C}_{20} \mathrm{H}_{25} \mathrm{~N}_{2} \mathrm{O}_{5} \mathrm{~S}[\mathrm{M}+\mathrm{H}]^{+}$, 405.1484. Found 405.1502.<smiles>CCOC(=O)N[C@H](c1ccccc1)C(C)/C(=N\Sc1ccccc1)OCC</smiles>

\section{iso-Propyl}

anti- $N$-(benzenesulfonyl)-3(ethoxycarbonylamino)- 2-methyl-3-phenylpropionimidate (anti-7b): Mp. $78{ }^{\circ} \mathrm{C} ;{ }^{1} \mathrm{H} \mathrm{NMR}\left(\mathrm{CDCl}_{3}\right) ; \delta=8.07-8.13(\mathrm{~m}, 2 \mathrm{H})$, $7.40(\mathrm{~d}, 2 \mathrm{H}, J=6.9 \mathrm{~Hz}), 7.07(\mathrm{t}, 2 \mathrm{H}, J=6.9 \mathrm{~Hz}), 6.98-7.04(\mathrm{~m}$, $4 \mathrm{H}), 6.77(\mathrm{~d}, 1 \mathrm{H}, J=9.6 \mathrm{~Hz}), 5.09(\mathrm{t}, 1 \mathrm{H}, J=11.0 \mathrm{~Hz}$ ), 4.83 (quint, $1 \mathrm{H}, J=6.2 \mathrm{~Hz}$ ), $4.41(\mathrm{qd}, 1 \mathrm{H}, J=11.0,6.9 \mathrm{~Hz}), 3.85-4.02(\mathrm{~m}, 2 \mathrm{H}), 1.08(\mathrm{~d}, 3 \mathrm{H}, J=6.2 \mathrm{~Hz}), 0.98(\mathrm{~d}$, $3 \mathrm{H}, J=6.2 \mathrm{~Hz}), 0.88-94(\mathrm{~m}, 6 \mathrm{H}) ;{ }^{13} \mathrm{C} \mathrm{NMR}\left(\mathrm{CDCl}_{3}\right) \delta=176.8,155.7,143.0,141.1$, 132.4, 129.0, 128.9, 128.2, 127.7, 126.9, 72.5, 60.7, 59.2, 45.7, 20.8, 20.4, 14.9, 14.6; IR (neat) 3063, 3033, 2983, 2938, 2880, 1724, 1593, 1520, 1481, 1456, 1448, 1385, 1374, 1361, 1302, 1245, 1222, 1154, 1091, 1056, 1026, 1000, 974, 929, 909, 852, 829, 769, 758, 738, 702, 689, 671, 636, 597, 566, 537, 504, 470, $418 \mathrm{~cm}^{-1}$; HRMS (FAB); Exact mass calcd for $\mathrm{C}_{22} \mathrm{H}_{29} \mathrm{~N}_{2} \mathrm{O}_{5} \mathrm{~S}[\mathrm{M}+\mathrm{H}]^{+}, 433.1797$. Found 433.1803.

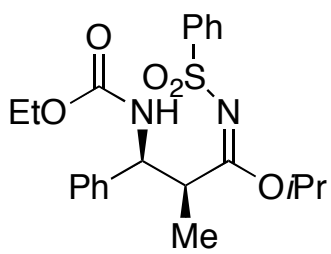

iso-Propyl

syn- $N$-(benzenesulfonyl)-3(ethoxycarbonylamino)-2-methyl-3-phenylpropionimidate (syn-7b): Mp. $145-147{ }^{\circ} \mathrm{C} ;{ }^{1} \mathrm{H} \mathrm{NMR}\left(\mathrm{CDCl}_{3}\right) ; \delta=7.97-8.02$ $(\mathrm{m}, 2 \mathrm{H}), 7.54(\mathrm{~d}, 2 \mathrm{H}, J=6.9 \mathrm{~Hz}), 7.13-7.18(\mathrm{~m}, 3 \mathrm{H}), 7.01(\mathrm{t}$, $1 \mathrm{H}, J=7.5 \mathrm{~Hz}), 6.93-6.98(\mathrm{~m}, 2 \mathrm{H}), 5.29(\mathrm{t}, 1 \mathrm{H}, J=9.7 \mathrm{~Hz}), 4.52(\mathrm{~d}, 1 \mathrm{H}, J=9.7 \mathrm{~Hz})$, 4.46 (quint, $1 \mathrm{H}, J=6.2 \mathrm{~Hz}$ ), 4.34 (dq, $1 \mathrm{H}, J=9.7,7.7 \mathrm{~Hz}$ ), 3.86-4.04 (m, 2H), 1.54 (d, 
$3 \mathrm{H}, J=6.9 \mathrm{~Hz}), 0.95(\mathrm{t}, 3 \mathrm{H}, J=6.9 \mathrm{~Hz}), 0.71(\mathrm{~d}, 3 \mathrm{H}, J=6.2 \mathrm{~Hz}), 0.45(\mathrm{~d}, 3 \mathrm{H}, J=5.5$ $\mathrm{Hz}) ;{ }^{13} \mathrm{C} \mathrm{NMR}\left(\mathrm{CDCl}_{3}\right) \delta=175.2,156.3,143.4,141.4,132.0,128.8,128.7,128.3$, 126.8, 71.8, 60.8, 57.5, 44.2, 20.6, 20.0, 16.0, 14.5; IR (neat) 3063, 3033, 2982, 2936, 2876, 1698, 1596, 1532, 1481, 1447, 1448, 1382, 1355, 1305, 1286, 1262, 1236, 1157, 1093, 1082, 1034, 1001, 980, 929, 908, 844, 766, 737, 702, 689, 638, 587, 540, 418 $\mathrm{cm}^{-1}$; HRMS (FAB); Exact mass calcd for $\mathrm{C}_{22} \mathrm{H}_{29} \mathrm{~N}_{2} \mathrm{O}_{5} \mathrm{~S}[\mathrm{M}+\mathrm{H}]^{+}, 433.1797$. Found 433.1779.

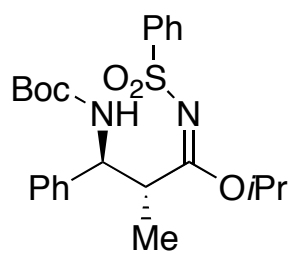

iso-Propyl anti- $N$-(benzenesulfonyl)-3-(tertbutoxycarbonylamino)-2-methyl-3-phenylpropionimidate (anti-7c): ${ }^{1} \mathrm{H} \mathrm{NMR}\left(\mathrm{C}_{6} \mathrm{D}_{6}\right) \delta=8.14(\mathrm{~d}, 2 \mathrm{H}, J=6.8 \mathrm{~Hz}), 7.38(\mathrm{~d}$, $2 \mathrm{H}, J=7.4 \mathrm{~Hz}), 6.95-7.07(\mathrm{~m}, 6 \mathrm{H}), 6.66(\mathrm{~d}, 1 \mathrm{H}, J=9.6 \mathrm{~Hz}), 5.06$ $(\mathrm{t}, 1 \mathrm{H}, J=10.5 \mathrm{~Hz}), 4.80-4.86(\mathrm{~m}, 1 \mathrm{H}), 4.32-4.43(\mathrm{~m}, 1 \mathrm{H}), 1.34(\mathrm{~s}, 9 \mathrm{H}), 1.12(\mathrm{~d}, 3 \mathrm{H}$, $J=6.2 \mathrm{~Hz}), 0.95(\mathrm{~d}, 3 \mathrm{H}, J=6.8 \mathrm{~Hz}), 0.91(\mathrm{~d}, 3 \mathrm{H}, J=6.2 \mathrm{~Hz}) ;{ }^{13} \mathrm{C} \mathrm{NMR}\left(\mathrm{C}_{6} \mathrm{D}_{6}\right) \delta=$ 17.67, 154.9, 143.2, 132.3, 129.0, 128.8, 127.6, 126.9, 78.7, 72.5, 58.8, 45.8, 28.4, 20.8, 20.6, 14.9; IR (neat) 3387, 2979, 2938, 1717, 1592, 1508, 1455, 1386, 1364, 1302, 1247, 1155, 1090, 1054, 1002, 973, 909, 851, 757, 737, 700, 688, 635, 590, 538 $\mathrm{cm}^{-1}$; HRMS (FAB); Exact mass calcd for $\mathrm{C}_{24} \mathrm{H}_{33} \mathrm{~N}_{2} \mathrm{O}_{5} \mathrm{~S}[\mathrm{M}+\mathrm{H}]^{+}, 461.2110$. Found 461.2099.

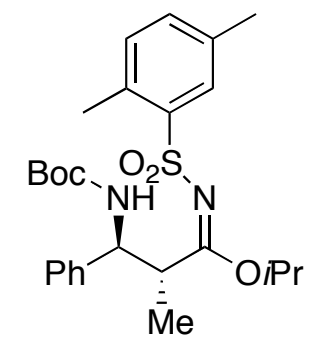

\section{iso-Propyl anti-3-(tert-butoxycarbonylamino)-2-methyl-3-} phenyl- $N$-(2,5-xylylsulfonyl)propionimidate (anti-7d): $\mathrm{Mp}$. $122-123{ }^{\circ} \mathrm{C} ;{ }^{1} \mathrm{H}$ NMR $\left(\mathrm{CDCl}_{3}\right) ; \delta=8.19(\mathrm{~s}, 1 \mathrm{H}), 7.36(\mathrm{~d}, 2 \mathrm{H}, J=$ $6.8 \mathrm{~Hz}), 6.93-7.05(\mathrm{~m}, 4 \mathrm{H}), 6.89(\mathrm{~d}, 1 \mathrm{H}, J=7.3 \mathrm{~Hz}), 6.62(\mathrm{~d}, 1 \mathrm{H}, J$ $=9.6 \mathrm{~Hz}$ ), $5.03(\mathrm{t}, 1 \mathrm{H}, J=10.5 \mathrm{~Hz}), 4.87$ (quint, $1 \mathrm{H}, J=6.2 \mathrm{~Hz}$ ), $4.48(\mathrm{sext}, 1 \mathrm{H}, J=5.9 \mathrm{~Hz}), 2.86(\mathrm{~s}, 3 \mathrm{H}), 1.96(\mathrm{~s}, 3 \mathrm{H}), 1.34(\mathrm{~s}, 9 \mathrm{H})$, $1.15(\mathrm{~d}, 3 \mathrm{H}, J=5.7 \mathrm{~Hz}), 0.98(\mathrm{~d}, 3 \mathrm{H}, J=6.2 \mathrm{~Hz}), 0.91(\mathrm{~d}, 3 \mathrm{H}, J=6.2 \mathrm{~Hz}) ;{ }^{13} \mathrm{C} \mathrm{NMR}$ $\left(\mathrm{CDCl}_{3}\right) \delta=177.5,154.7,141.4,140.5,136.1,134.5,133.4,132.4,128.9,128.3$, 127.9, 127.7, 78.6, 72.1, 58.9, 46.0, 28.4, 21.0, 21.0, 20.6, 20.6, 14.9; IR (neat) 3060, 3032, 2841, 2936, 2881, 1715, 1588, 1513, 1495, 1456, 1389, 1365, 1299, 1267, 1248, 1225, 1155, 1102, 1066, 1053, 1004, 973, 930, 910, 884, 851, 824, 738, 707, 644, 599, 553, 499, $465 \mathrm{~cm}^{-1}$; HRMS (ESI); Exact mass calcd for $\mathrm{C}_{26} \mathrm{H}_{37} \mathrm{~N}_{2} \mathrm{O}_{5} \mathrm{~S}[\mathrm{M}+\mathrm{H}]^{+}$, 489.2423. Found 489.2417. 


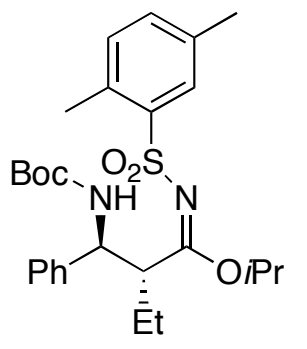

iso-Propyl anti-3-(tert-butoxycarbonylamino)-2-ethyl-3-phenyl$N$-(2,5-xylylsulfonyl)propionimidate (anti-7e): Mp. $95-96{ }^{\circ} \mathrm{C}$; ${ }^{1} \mathrm{H}$ NMR $\left(\mathrm{CDCl}_{3}\right) ; \delta=8.18(\mathrm{~s}, 1 \mathrm{H}), 7.44(\mathrm{~d}, 2 \mathrm{H}, J=7.4 \mathrm{~Hz}), 6.93$ $7.08(\mathrm{~m}, 4 \mathrm{H}), 6.80-6.92(\mathrm{~m}, 2 \mathrm{H}), 5.08(\mathrm{t}, 1 \mathrm{H}, J=10.5 \mathrm{~Hz}), 4.93$ (quint, $1 \mathrm{H}, J=6.2 \mathrm{~Hz}), 4.32(\mathrm{td}, 1 \mathrm{H}, J=11.4,4.0 \mathrm{~Hz}), 2.87(\mathrm{~s}$, $3 \mathrm{H}), 1.95(\mathrm{~s}, 3 \mathrm{H}), 1.59-1.65(\mathrm{~m}, 1 \mathrm{H}), 1.34(\mathrm{~s}, 9 \mathrm{H}), 1.20-1.40(\mathrm{~m}$, $1 \mathrm{H}), 1.16(\mathrm{~d}, 3 \mathrm{H}, J=6.2 \mathrm{~Hz}), 0.88-0.95(\mathrm{~m}, 6 \mathrm{H}) ;{ }^{13} \mathrm{C} \mathrm{NMR}\left(\mathrm{CDCl}_{3}\right) \delta=176.9,153.8$, $141.9,140.6,136.1,134.5,133.4,53.7,58.4,132.4,129.0,128.9,128.3,127.7,78.6$, 72.0, 28.4, 23.9, 21.1, 21.0, 20.7, 20.6, 11.8; IR (neat) 3062, 3031, 2978, 2934, 2878, $2717,1717,1586,1509,1496,1458,1390,1365,1297,1251,1202,1154,1100,1065$, $1011,991,915,885,865,837,822,774,747,707,642,599,553,532,499,464,437$, $419 \mathrm{~cm}^{-1}$; HRMS (FAB); Exact mass calcd for $\mathrm{C}_{27} \mathrm{H}_{39} \mathrm{~N}_{2} \mathrm{O}_{5} \mathrm{~S}[\mathrm{M}+\mathrm{H}]^{+}, 503.2580$. Found 503.2554.

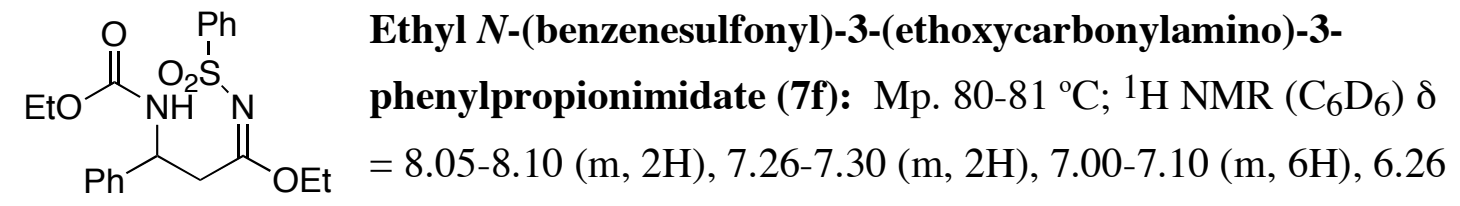
$(\mathrm{d}, 1 \mathrm{H}, J=9.1 \mathrm{~Hz}), 5.47-5.57(\mathrm{~m}, 1 \mathrm{H}), 3.92-4.03(\mathrm{~m}, 2 \mathrm{H}), 3.59-3.81(\mathrm{~m}, 3 \mathrm{H}), 2.95$ $(\mathrm{dd}, 1 \mathrm{H}, J=5.1,14.2 \mathrm{~Hz}), 0.95(\mathrm{t}, 3 \mathrm{H}, J=7.1 \mathrm{~Hz}), 0.82(\mathrm{t}, 3 \mathrm{H}, J=7.1 \mathrm{~Hz}) ;{ }^{13} \mathrm{C}$ NMR $\left(\mathrm{C}_{6} \mathrm{D}_{6}\right) \delta=172.8,155.9,142.7,142.0,132.4,129.0,128.8,127.0,126.6,64.8$, 60.8, 53.3, 41.1, 14.6, 13.2; IR (neat) 3360, 3065, 2982, 1719, 1599, 1523, 1473, 1446, 1397, 1374, 1305, 1243, 1155, 1091, 1041, 888, 755, 734, 700, 689, $631 \mathrm{~cm}^{-1}$; HRMS (FAB); Exact mass calcd for $\mathrm{C}_{20} \mathrm{H}_{25} \mathrm{~N}_{2} \mathrm{O}_{5} \mathrm{~S}[\mathrm{M}+\mathrm{H}]^{+}$, 405.1484. Found 405.1487 .

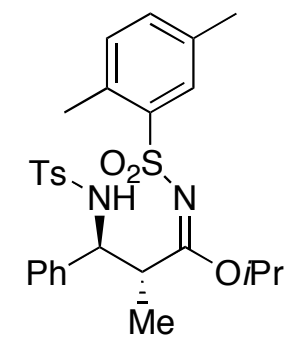

iso-Propyl anti-2-methyl-3-phenyl-3-(p-toluenesulfonylamino)$N$-(2,5-xylylsulfonyl)propionimidate (anti-7g): Mp. $140-142{ }^{\circ} \mathrm{C}$; ${ }^{1} \mathrm{H} \mathrm{NMR}\left(\mathrm{CDCl}_{3}\right) ; \delta=8.21(\mathrm{~s}, 1 \mathrm{H}), 7.34(\mathrm{~d}, 2 \mathrm{H}, J=7.9 \mathrm{~Hz}), 6.98$ $(\mathrm{d}, 1 \mathrm{H}, J=7.9 \mathrm{~Hz}), 6.86-6.95(\mathrm{~m}, 3 \mathrm{H}), 6.70-6.86(\mathrm{~m}, 4 \mathrm{H}), 6.48(\mathrm{~d}$, $2 \mathrm{H}, J=8.5 \mathrm{~Hz}$ ), 5.02 (quint, $1 \mathrm{H}, J=6.2 \mathrm{~Hz}$ ), 4.69 (t, $1 \mathrm{H}, J=10.2$ $\mathrm{Hz}), 4.30(\mathrm{qd}, 1 \mathrm{H}, J=10.7,6.8 \mathrm{~Hz}), 3.11(\mathrm{~s}, 3 \mathrm{H}), 1.97(\mathrm{~s}, 3 \mathrm{H}), 1.81$ $(\mathrm{s}, 3 \mathrm{H}), 1.40(\mathrm{~d}, 3 \mathrm{H}, J=5.2 \mathrm{~Hz}), 1.00$ (d, 3H, $J=6.2 \mathrm{~Hz}), 0.93(\mathrm{~d}, 3 \mathrm{H}, J=6.8 \mathrm{~Hz})$; ${ }^{13} \mathrm{C}$ NMR $\left(\mathrm{CDCl}_{3}\right) \delta=176.9,141.8,140.2,139.3,138.6,136.1,135.0,133.6,132.6$, 128.9, 128.8, 128.6, 127.6, 127.0, 73.0 , 62.1 , 46.7, 21.4, 21.1, 20.9, 20.6, 20.5, 14.6; IR (neat) 3056, 2986, 2938, 2880, 1589, 1494, 1457, 1438, 1423, 1386, 1355, 1333, 1305, 1291, 1266, 1162, 1101, 1092, 1063, 975, 910, 896, 856, 822, 814, 738, 706, 
668, 645, 581, 565, 549, 500, 463, $418 \mathrm{~cm}^{-1}$; HRMS (FAB); Exact mass calcd for $\mathrm{C}_{28} \mathrm{H}_{35} \mathrm{~N}_{2} \mathrm{O}_{5} \mathrm{~S}_{2}[\mathrm{M}+\mathrm{H}]^{+}$, 543.1987. Found 543.1990.

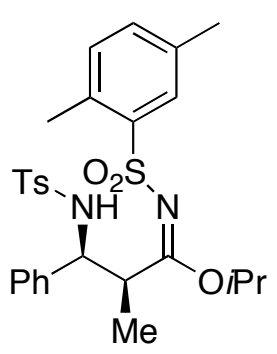

iso-Propyl syn-2-methyl-3-phenyl-3-(p-toluenesulfonylamino)$N$-(2,5-xylylsulfonyl)propionimidate (syn-7g): Mp. $160-161{ }^{\circ} \mathrm{C}$; ${ }^{1} \mathrm{H} \mathrm{NMR}\left(\mathrm{CDCl}_{3}\right) \delta=7.80(\mathrm{~s}, 1 \mathrm{H}), 7.46(\mathrm{~d}, 2 \mathrm{H}, J=8.5 \mathrm{~Hz}), 7.21$ $(\mathrm{d}, 1 \mathrm{H}, J=7.4 \mathrm{~Hz}), 7.11(\mathrm{~d}, 1 \mathrm{H}, J=7.9 \mathrm{~Hz}), 7.00-7.07(\mathrm{~m}, 7 \mathrm{H})$, $5.17(\mathrm{~d}, 1 \mathrm{H}, J=9.6 \mathrm{~Hz}), 4.68-4.77(\mathrm{~m}, 1 \mathrm{H}), 4.52(\mathrm{t}, 1 \mathrm{H}, J=9.4 \mathrm{~Hz})$, 4.10-4.17 (m, 1H), 2.39 (s, 3H), 2.35 (s, 3H), 2.31 (s, 3H), 1.44 (d, $3 \mathrm{H}, J=6.8 \mathrm{~Hz}), 1.14(\mathrm{~d}, 3 \mathrm{H}, J=6.2 \mathrm{~Hz}), 0.78(\mathrm{~d}, 3 \mathrm{H}, J=6.2 \mathrm{~Hz}) ;{ }^{13} \mathrm{C} \mathrm{NMR}\left(\mathrm{CDCl}_{3}\right)$ $\delta=17.46,142.9,139.5,138.5,137.8,135.6,134.2,133.1,132.0,129.2,128.1,128.0$, 127.6, 127.4, 127.0, 72.1, 60.3, 44.6, 21.4, 21.1, 20.8, 20.5, 19.6, 15.6; IR (neat) 3276, 3032, 2983, 2877, 1592, 1492, 1455, 1381, 1331, 1303, 1184, 1159, 1096, 1062, 981, 909, 813, 764, 733, 704, 669, 646, 607, 562, $511 \mathrm{~cm}^{-1}$; HRMS (FAB); Exact mass calcd for $\mathrm{C}_{28} \mathrm{H}_{35} \mathrm{~N}_{2} \mathrm{O}_{5} \mathrm{~S}_{2}[\mathrm{M}+\mathrm{H}]^{+}, 543.1987$. Found 543.1971.

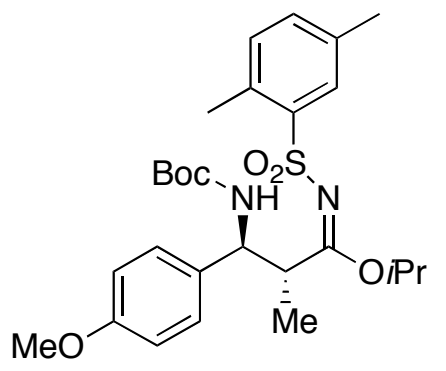

\section{iso-Propyl}

anti-3-(tert-butoxycarbonylamino)-3-( $p$ methoxyphenyl)-2-methyl- $N-(2,5-$ xylylsulfonyl)propionimidate (anti-7h): Mp. 142$145{ }^{\circ} \mathrm{C} ;{ }^{1} \mathrm{H}$ NMR $\left(\mathrm{CDCl}_{3}\right) ; \delta=8.20(\mathrm{~s}, 1 \mathrm{H}), 7.29(\mathrm{~d}, 2 \mathrm{H}, J$ $=9.1 \mathrm{~Hz}), 6.98(\mathrm{~d}, 1 \mathrm{H}, J=7.4 \mathrm{~Hz}), 6.89(\mathrm{~d}, 1 \mathrm{H}, J=7.4$ $\mathrm{Hz}), 6.55-6.65(\mathrm{~m}, 3 \mathrm{H}), 5.02(\mathrm{t}, 1 \mathrm{H}, J=9.5 \mathrm{~Hz}), 4.91$ (quint, $1 \mathrm{H}, J=6.2 \mathrm{~Hz}), 4.38(\mathrm{qd}, 1 \mathrm{H}, J=11.3,6.2 \mathrm{~Hz}), 3.27(\mathrm{~s}, 3 \mathrm{H}), 2.87(\mathrm{~s}, 3 \mathrm{H})$, $1.97(\mathrm{~s}, 3 \mathrm{H}), 1.36$ (s, 9H), 1.16 (d, 3H, $J=6.2 \mathrm{~Hz}), 1.04$ (d, 3H, $J=6.8 \mathrm{~Hz}), 0.93$ (d, $3 \mathrm{H}, J=6.2 \mathrm{~Hz}) ;{ }^{13} \mathrm{C} \mathrm{NMR}\left(\mathrm{CDCl}_{3}\right) \delta=177.7,159.6,154.7,140.6,136.1,134.6$, 133.4, 132.4, 131.8, 128.9, 128.7, 114.5, 78.6, 72.1, 58.4, 54.7, 46.2, 28.4, 21.0, 21.0, 20.6, 20.6, 15.0; IR (neat) 3055, 2984, 2937, 1713, 1586, 1513, 1457, 1422, 1386, 1365, 1299, 1265, 1155, 1101, 1054, 1036, 910, 832, 740, 706, 644, 609, $419 \mathrm{~cm}^{-1}$; HRMS (FAB); Exact mass calcd for $\mathrm{C}_{27} \mathrm{H}_{39} \mathrm{~N}_{2} \mathrm{O}_{6} \mathrm{~S}[\mathrm{M}+\mathrm{H}]^{+}, 519.2529$. Found 519.2512 .

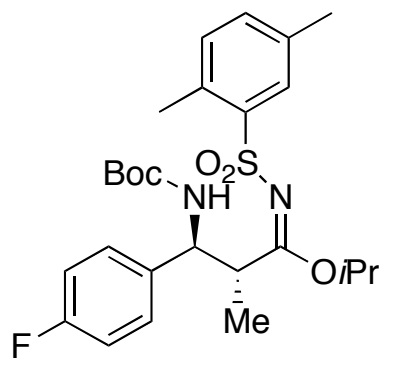

iso-Propyl

anti-3-(tert-butoxycarbonylamino)-3-( $p$ fluorophenyl)-2-methyl- $N-(2,5-$ xylylsulfonyl)propionimidate (anti-7i): Mp. $131-133{ }^{\circ} \mathrm{C}$; ${ }^{1} \mathrm{H} \mathrm{NMR}\left(\mathrm{CDCl}_{3}\right) ; \delta=8.19$ (s, 1H), 7.12-7.21 (m, 2H), 6.96 $(\mathrm{d}, 1 \mathrm{H}, J=7.4 \mathrm{~Hz}), 6.88(\mathrm{~d}, 1 \mathrm{H}, J=7.4 \mathrm{~Hz}), 6.59-6.69(\mathrm{~m}$, 2H), 4.95 (t, 1H, $J=10.2 \mathrm{~Hz}$ ), 4.87 (quint, $1 \mathrm{H}, J=6.0 \mathrm{~Hz}$ ), 
4.29 (sext, 1H, $J=6.4 \mathrm{~Hz}), 2.85$ (s, 3H), $1.95(\mathrm{~s}, 3 \mathrm{H}), 1.35$ (s, 9H), 1.14 (d, 3H, $J=$ $5.6 \mathrm{~Hz}), 0.95(\mathrm{~d}, 3 \mathrm{H}, J=6.2 \mathrm{~Hz}), 0.91(\mathrm{~d}, 3 \mathrm{H}, J=6.2 \mathrm{~Hz}) ;{ }^{13} \mathrm{C} \mathrm{NMR}\left(\mathrm{CDCl}_{3}\right) \delta=$ $177.2,160.6\left(\mathrm{~d}, J_{\mathrm{C}-\mathrm{F}}=244.3 \mathrm{~Hz}\right), 154.7,140.4,137.1,136.2,134.5,133.5,132.4$, $129.3\left(\mathrm{~d},{ }^{3} J_{\mathrm{C}-\mathrm{F}}=8.4 \mathrm{~Hz}\right), 128.9,115.7\left(\mathrm{~d},{ }^{2} J_{\mathrm{C}-\mathrm{F}}=21.5 \mathrm{~Hz}\right), 78.8,72.1,58.2,46.0,28.4$, 21.0, 21.0, 20.6, 14.8; IR (neat) 3056, 2984, 2933, 1714, 1588, 1542, 1457, 1366, 1298, 1266, 1226, 1156, 1096, 1055, 837, 741, 706, 644, 507, 527, $499 \mathrm{~cm}^{-1}$; HRMS (FAB); Exact mass calcd for $\mathrm{C}_{26} \mathrm{H}_{36} \mathrm{~N}_{2} \mathrm{O}_{5} \mathrm{SF}[\mathrm{M}+\mathrm{H}]^{+}$, 507.2329. Found 507.2326.

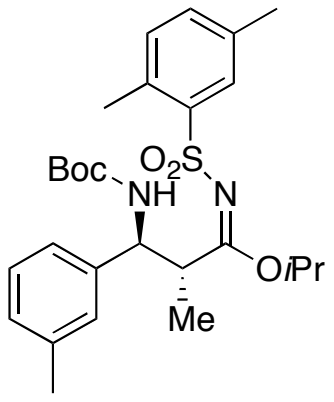

iso-Propyl

anti-3-(tert-butoxycarbonylamino)-3-(mmethylphenyl)-2-methyl- $N-(2,5-$

xylylsulfonyl)propionimidate (anti-7j): Mp. $110-112{ }^{\circ} \mathrm{C} ;{ }^{1} \mathrm{H}$ NMR $\left(\mathrm{CDCl}_{3}\right) ; \delta=8.18(\mathrm{~s}, 1 \mathrm{H}), 7.20-7.27(\mathrm{~m}, 2 \mathrm{H}), 6.98(\mathrm{dd}$, $2 \mathrm{H}, J=16.1,8.2 \mathrm{~Hz}), 6.90(\mathrm{~d}, 1 \mathrm{H}, J=6.8 \mathrm{~Hz}), 6.85(\mathrm{~d}, 1 \mathrm{H}, J=$ $7.4 \mathrm{~Hz}), 6.62(\mathrm{~d}, 1 \mathrm{H}, J=9.6 \mathrm{~Hz}), 5.04(\mathrm{t}, 1 \mathrm{H}, J=10.5 \mathrm{~Hz}), 4.90$ (quint, $1 \mathrm{H}, J=6.2 \mathrm{~Hz}), 4.40(\mathrm{qd}, 1 \mathrm{H}, J=11.1,6.2 \mathrm{~Hz}), 2.86(\mathrm{~s}$, $3 \mathrm{H}), 2.02(\mathrm{~s}, 3 \mathrm{H}), 1.98(\mathrm{~s}, 3 \mathrm{H}), 1.34(\mathrm{~s}, 9 \mathrm{H}), 1.17(\mathrm{~d}, 3 \mathrm{H}, J=6.2 \mathrm{~Hz}), 1.02$ (d, 3H, $J=$ $6.2 \mathrm{~Hz}), 0.93(\mathrm{~d}, 3 \mathrm{H}, J=6.2 \mathrm{~Hz}) ;{ }^{13} \mathrm{C} \mathrm{NMR}\left(\mathrm{CDCl}_{3}\right) \delta=177.6,154.7,141.4,138.6$, 136.1, 133.4, 132.4, 129.0, 128.4, 128.3, 124.7, 78.6, 72.1, 58.9, 46.1, 28.4, 28.3, 21.3, 21.1, 21.0, 20.6, 15.0; IR (neat) 3056, 2982, 2936, 2880, 1714, 1588, 1508, 1495, 1457, 1386, 1366, 1298, 1266, 1155, 1102, 1054, 1007, 974, 942, 908, 885, 846, 822, 791, 740, 708, 644, 606, 554, 499, 445, $419 \mathrm{~cm}^{-1}$; HRMS (FAB); Exact mass calcd for $\mathrm{C}_{27} \mathrm{H}_{39} \mathrm{~N}_{2} \mathrm{O}_{5} \mathrm{~S}[\mathrm{M}+\mathrm{H}]^{+}$, 503.2580. Found 503.2554.

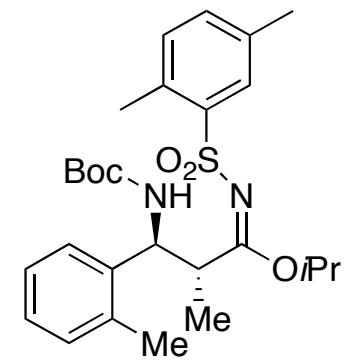

iso-Propyl

xylylsulfonyl)propionimidate (anti-7k): ${ }^{1} \mathrm{H}$ NMR $\left(\mathrm{CDCl}_{3}\right) ; \delta$ $=8.20(\mathrm{~s}, 1 \mathrm{H}), 7.58(\mathrm{~d}, 1 \mathrm{H}, J=7.3 \mathrm{~Hz}), 6.82-7.07(\mathrm{~m}, 5 \mathrm{H}), 6.64$ $(\mathrm{d}, 1 \mathrm{H}, J=9.1 \mathrm{~Hz}), 5.44(\mathrm{t}, 1 \mathrm{H}, J=10.5 \mathrm{~Hz}$ ), 4.90 (quint, $1 \mathrm{H}, J$ $=6.2 \mathrm{~Hz}), 4.46(\mathrm{qd}, 1 \mathrm{H}, J=11.3,6.8 \mathrm{~Hz}), 2.87(\mathrm{~s}, 3 \mathrm{H}), 2.52(\mathrm{~s}$, $3 \mathrm{H}), 1.97$ (s, 3H), 1.32 (s, 9H), 1.15 (d, 3H, $J=6.2 \mathrm{~Hz}), 1.00$ (d, 3H, $J=6.8 \mathrm{~Hz}), 0.90$ $(\mathrm{d}, 3 \mathrm{H}, J=6.2 \mathrm{~Hz}) ;{ }^{13} \mathrm{C} \mathrm{NMR}\left(\mathrm{CDCl}_{3}\right) \delta=177.5,154.9,140.6,140.3,136.5,136.1$, 134.5, 133.4, 132.4, 130.6, 128.9, 127.6, 127.4, 126.5, 78.6, 72.1, 46.9, 26.4, 21.1, 21.0, 20.6, 20.6, 19.9, 14.1; IR (neat) 3063, 2980, 2933, 2880, 1715, 1591, 1509, 1494, 1458, 1385, 1364, 1299, 1248, 1233, 1155, 1102, 1056, 1003, 971, 955, 910, 884, 850, 822, 793, 760, 748, 734, 708, 643, 601, 583, 556, 518, 500, 459, $412 \mathrm{~cm}^{-1}$; Exact mass calcd for $\mathrm{C}_{27} \mathrm{H}_{39} \mathrm{~N}_{2} \mathrm{O}_{5} \mathrm{~S}[\mathrm{M}+\mathrm{H}]^{+}$, 503.2580. Found 503.2554. 


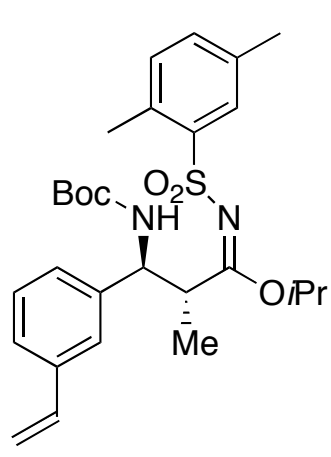

iso-Propyl anti-3-(tert-butoxycarbonylamino)-3-(mvinylphenyl)-2-methyl- $N$-(2,5-xylylsulfonyl)propionimidate (anti-7l): Mp. 87-91 ${ }^{\circ} \mathrm{C} ;{ }^{1} \mathrm{H} \mathrm{NMR}\left(\mathrm{CDCl}_{3}\right) ; \delta=8.19(\mathrm{~s}, 1 \mathrm{H})$, $7.52(\mathrm{~s}, 1 \mathrm{H}), 7.28(\mathrm{~d}, 1 \mathrm{H}, J=7.9 \mathrm{~Hz}), 7.10(\mathrm{~d}, 1 \mathrm{H}, J=7.4 \mathrm{~Hz})$, $6.98(\mathrm{t}, 1 \mathrm{H}, J=7.9 \mathrm{~Hz}), 6.96(\mathrm{~d}, 1 \mathrm{H}, J=7.4 \mathrm{~Hz}), 6.88(\mathrm{~d}, 1 \mathrm{H}, J=$ $7.9 \mathrm{~Hz}), 6.67(\mathrm{~d}, 1 \mathrm{H}, J=9.6 \mathrm{~Hz}), 6.46(\mathrm{dd}, 1 \mathrm{H}, J=17.6,10.7$ $\mathrm{Hz}), 5.57(\mathrm{~d}, 1 \mathrm{H}, J=17.6 \mathrm{~Hz}), 5.07(\mathrm{t}, 1 \mathrm{H}, J=10.2 \mathrm{~Hz}), 5.00(\mathrm{~d}$, $1 \mathrm{H}, J=10.7 \mathrm{~Hz}$ ), 4.88 (quint, $1 \mathrm{H}, J=6.2 \mathrm{~Hz}$ ), 4.41 (qd, $1 \mathrm{H}, J=$ 11.3, $6.8 \mathrm{~Hz}$ ), 2.86 (s, 3H), 1.96 (s, 3H), 1.34 (s, 9H), 1.16 (d, 3H, $J=6.2 \mathrm{~Hz}), 0.98$ (d, $3 \mathrm{H}, J=6.8 \mathrm{~Hz}), 0.92(\mathrm{~d}, 3 \mathrm{H}, J=6.2 \mathrm{~Hz}) ;{ }^{13} \mathrm{C} \mathrm{NMR}\left(\mathrm{CDCl}_{3}\right) \delta=177.4,154.7,141.8$, $140.5,138.4$, 136.9, 136.2, 134.5, 133.4, 132.4, 129.4, 128.9, 126.8, 126.1, 125.7, 114.1, 78.7, 72.1, 58.9, 46.1, 28.4, 21.0, 20.6, 20.6, 14.9; IR (neat) 3087, 3048, 2981, 2933, 2879, 1718, 1602, 1507, 1457, 1389, 1363, 1309, 1250, 1140, 1092, 1055, 1004, 973, 908, 857, 844, 804, 784, 748, 708, 682, 643, 600, 552, 503, 460, $412 \mathrm{~cm}^{-1}$; HRMS (FAB); Exact mass calcd for $\mathrm{C}_{28} \mathrm{H}_{39} \mathrm{~N}_{2} \mathrm{O}_{5} \mathrm{~S}[\mathrm{M}+\mathrm{H}]^{+}, 515.2580$. Found 515.2570 .

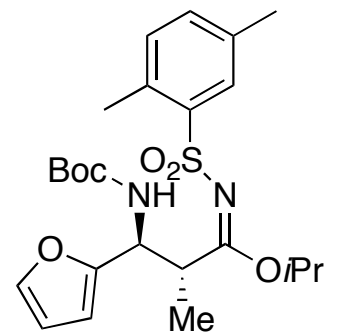

iso-Propyl anti-3-(tert-butoxycarbonylamino)-3-(2-furyl)-2methyl- $N$-(2,5-xylylsulfonyl)propionimidate (anti-7m): $\mathrm{Mp}$. $84{ }^{\circ} \mathrm{C} ;{ }^{1} \mathrm{H} \mathrm{NMR}\left(\mathrm{CDCl}_{3}\right) ; \delta=8.17(\mathrm{~s}, 1 \mathrm{H}), 6.90-6.97(\mathrm{~m}, 2 \mathrm{H})$, $6.87(\mathrm{~d}, 1 \mathrm{H}, J=7.4 \mathrm{~Hz}), 6.18(\mathrm{~d}, 1 \mathrm{H}, J=9.6 \mathrm{~Hz}), 6.09$ (d, 1H, $J$ $=2.7 \mathrm{~Hz}), 5.90-5.94(\mathrm{~m}, 1 \mathrm{H}), 5.21(\mathrm{t}, 1 \mathrm{H}, J=10.2 \mathrm{~Hz}), 4.87$ (quint, $1 \mathrm{H}, J=6.2 \mathrm{~Hz}), 4.59(\mathrm{qd}, 1 \mathrm{H}, J=11.3,6.8 \mathrm{~Hz}), 2.82(\mathrm{~s}$, $3 \mathrm{H}), 1.95(\mathrm{~s}, 3 \mathrm{H}), 1.34(\mathrm{~s}, 9 \mathrm{H}), 1.13(\mathrm{~d}, 3 \mathrm{H}, J=6.2 \mathrm{~Hz}), 1.06(\mathrm{~d}, 3 \mathrm{H}, J=6.7 \mathrm{~Hz}), 0.89$ $(\mathrm{d}, 3 \mathrm{H}, J=6.2 \mathrm{~Hz}) ;{ }^{13} \mathrm{C} \mathrm{NMR}\left(\mathrm{CDCl}_{3}\right) \delta=176.6,154.6,153.1,140.6,136.1,134.6$, 133.3, 132.4, 128.9, 110.3, 108.1, 78.8, 72.1, 52.1, 43.7, 28.3, 21.0, 21.0, 20.6, 20.5, 14.8; IR (neat) 3139, 3121, 3056, 2980, 2936, 2879, 1715, 1583, 1509, 1456, 1390, 1367, 1299, 1256, 1200, 1155, 1137, 1103, 1064, 1051, 1010, 971, 910, 884, 860, 848, 822, 798, 754, 731, 709, 680, 644, 592, 556, 498, $424 \mathrm{~cm}^{-1}$; HRMS (FAB); Exact mass calcd for $\mathrm{C}_{24} \mathrm{H}_{35} \mathrm{~N}_{2} \mathrm{O}_{6} \mathrm{~S}[\mathrm{M}+\mathrm{H}]^{+}$, 479.2216. Found 479.2225.

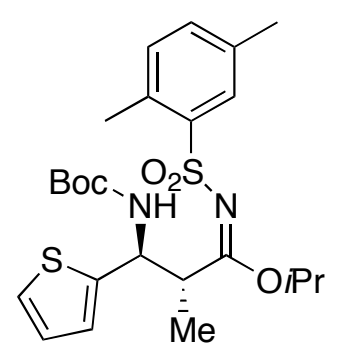

iso-Propyl anti-3-(tert-butoxycarbonylamino)-2-methyl-3-(2thienyl)- $N$-(2,5-xylylsulfonyl)propionimidate (anti-7n): $\mathrm{Mp}$. 104-107 ${ }^{\circ} \mathrm{C} ;{ }^{1} \mathrm{H}$ NMR $\left(\mathrm{CDCl}_{3}\right) ; \delta=8.17(\mathrm{~s}, 1 \mathrm{H}), 6.85-7.00(\mathrm{~m}$, $3 \mathrm{H}), 6.76(\mathrm{~d}, 1 \mathrm{H}, J=4.6 \mathrm{~Hz}), 6.61(\mathrm{dd}, 1 \mathrm{H}, J=5.1,3.4 \mathrm{~Hz})$, $6.32(\mathrm{~d}, 1 \mathrm{H}, J=9.1 \mathrm{~Hz}), 5.35(\mathrm{t}, 1 \mathrm{H}, J=10.5 \mathrm{~Hz}), 4.87$ (quint, $1 \mathrm{H}, J=6.2 \mathrm{~Hz}), 4.59(\mathrm{qd}, 1 \mathrm{H}, J=10.8,6.8 \mathrm{~Hz}), 2.81(\mathrm{~s}, 3 \mathrm{H})$, 
$1.96(\mathrm{~s}, 3 \mathrm{H}), 1.33(\mathrm{~s}, 9 \mathrm{H}), 1.10(\mathrm{~d}, 6 \mathrm{H}, J=6.8 \mathrm{~Hz}), 0.88(\mathrm{~d}, 3 \mathrm{H}, J=6.2 \mathrm{~Hz}) ;{ }^{13} \mathrm{C}$ $\operatorname{NMR}\left(\mathrm{CDCl}_{3}\right) \delta=176.7,154.6,144.7,140.5,136.1,134.5,133.4,132.4,128.9$, 127.1, 125.6, 124.8, 78.9, 72.1, 54.0, 46.3, 26.4, 21.0, 20.6, 20.5, 15.2; IR (neat) 3056, 2983, 2934, 2880, 1714, 1588, 1507, 1494, 1456, 1420, 1387, 1366, 1299, 1265, 1233 , 1155, 1101, 1054, 1003, 973, 909, 850, 822, 739, 707, 644, 607, 553, 503, $412 \mathrm{~cm}^{-1}$; HRMS (FAB); Exact mass calcd for $\mathrm{C}_{24} \mathrm{H}_{35} \mathrm{~N}_{2} \mathrm{O}_{5} \mathrm{~S}_{2}[\mathrm{M}+\mathrm{H}]^{+}, 495.1987$. Found 495.1980 .

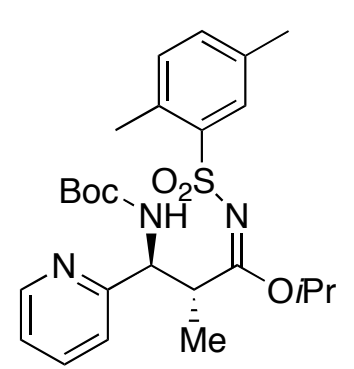

iso-Propyl anti-3-(tert-butoxycarbonylamino)-2-methyl-3-(2pyridyl)- $N$-(2,5-xylylsulfonyl)propionimidate (anti-7o): $\mathrm{Mp}$. $113-115{ }^{\circ} \mathrm{C} ;{ }^{1} \mathrm{H}$ NMR $\left(\mathrm{CDCl}_{3}\right) ; \delta=8.79(\mathrm{~s}, 1 \mathrm{H}), 8.35-8.45(\mathrm{~m}$, $1 \mathrm{H}), 8.17(\mathrm{~s}, 1 \mathrm{H}), 7.46(\mathrm{~d}, 1 \mathrm{H}, J=7.9 \mathrm{~Hz}), 6.96(\mathrm{~d}, 1 \mathrm{H}, J=7.4$ $\mathrm{Hz}), 6.87(\mathrm{~d}, 1 \mathrm{H}, J=7.4 \mathrm{~Hz}), 6.60-6.73(\mathrm{~m}, 2 \mathrm{H}), 5.00(\mathrm{t}, 1 \mathrm{H}, J$ $=9.9 \mathrm{~Hz}), 4.84$ (quint, $1 \mathrm{H}, J=6.2 \mathrm{~Hz}), 4.27(\mathrm{qd}, 1 \mathrm{H}, J=11.3$, $6.8 \mathrm{~Hz}), 2.83(\mathrm{~s}, 3 \mathrm{H}), 1.96(\mathrm{~s}, 3 \mathrm{H}), 1.33(\mathrm{~s}, 9 \mathrm{H}), 1.11(\mathrm{~d}, 3 \mathrm{H}, J=6.2 \mathrm{~Hz}), 0.88(\mathrm{~d}, 3 \mathrm{H}$, $J=6.2 \mathrm{~Hz}), 0.87(\mathrm{~d}, 3 \mathrm{H}, J=6.2 \mathrm{~Hz}) ;{ }^{13} \mathrm{C} \mathrm{NMR}\left(\mathrm{CDCl}_{3}\right) \delta=176.9,154.7,150.0$, $149.7,140.3,136.4,136.2,134.5,133.8,133.6,132.4,128.9,123.9,79.0,72.3,56.6$, 45.6, 28.4, 21.0, 20.9, 20.6, 14.7; IR (neat) 3059, 2980, 2934, 2880, 1715, 1591, 1507, 1495, 1456, 1429, 1390, 1366, 1298, 1255, 1154, 1101, 1054, 1027, 1005, 973, 910, 883, 852, 820, 779, 735, 708, 643, 602, 554, 499, 460, $412 \mathrm{~cm}^{-1}$; HRMS (FAB); Exact mass calcd for $\mathrm{C}_{25} \mathrm{H}_{36} \mathrm{~N}_{3} \mathrm{O}_{5} \mathrm{~S}[\mathrm{M}+\mathrm{H}]^{+}$, 490.2376. Found 490.2356.

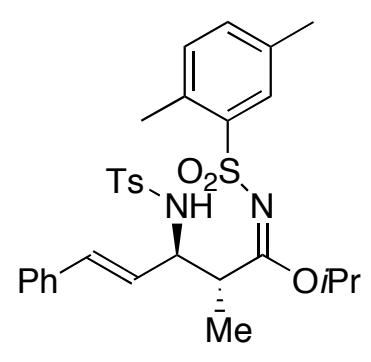

\section{iso-Propyl}

anti-2-methyl-5-phenyl-3- $(p-$ toluenesulfonylamino)- $N$-(2,5-xylylsulfonyl)pent-4enimidate (anti-7p): Mp. $125-128{ }^{\circ} \mathrm{C} ;{ }^{1} \mathrm{H} \mathrm{NMR}\left(\mathrm{CDCl}_{3}\right) ; \delta$ $=8.22(\mathrm{~s}, 1 \mathrm{H}), 7.67(\mathrm{~d}, 2 \mathrm{H}, J=7.9 \mathrm{~Hz}), 6.98-7.04(\mathrm{~m}, 3 \mathrm{H})$, $6.95(\mathrm{~d}, 1 \mathrm{H}, J=7.9 \mathrm{~Hz}), 6.88(\mathrm{dd}, 1 \mathrm{H}, J=7.4,1.1 \mathrm{~Hz}), 6.73$ $(\mathrm{dd}, 2 \mathrm{H}, J=7.6,2.0 \mathrm{~Hz}), 6.58(\mathrm{~d}, 2 \mathrm{H}, J=7.9 \mathrm{~Hz}), 6.37(\mathrm{~d}$,

$1 \mathrm{H}, J=9.1 \mathrm{~Hz}), 5.84(\mathrm{~d}, 1 \mathrm{H}, J=15.9 \mathrm{~Hz}), 5.24(\mathrm{dd}, 1 \mathrm{H}, J=15.9,9.1 \mathrm{~Hz}), 5.00$ (quint, $1 \mathrm{H}, J=6.2 \mathrm{~Hz}), 4.27(\mathrm{q}, 1 \mathrm{H}, J=9.8 \mathrm{~Hz}), 4.30(\mathrm{qd}, 1 \mathrm{H}, J=10.8,6.8 \mathrm{~Hz}), 2.97(\mathrm{~s}, 3 \mathrm{H})$, 1.97 (s, 3H), 1.70 (s, 3H), 1.37 (d, 3H, $J=6.2 \mathrm{~Hz}), 1.12$ (d, 3H, $J=6.2 \mathrm{~Hz}), 0.98$ (d, $3 \mathrm{H}, J=6.2 \mathrm{~Hz}) ;{ }^{13} \mathrm{C} \mathrm{NMR}\left(\mathrm{CDCl}_{3}\right) \delta=176.7,142.4,140.3,139.8,136.2,136.0$, 134.9, 134.3, 133.5, 132.5, 129.6, 129.3, 128.8, 128.3, 127.6, 126.8, 126.2 , 72.9, 60.7, 44.7, 21.4, 21.1, 20.9, 20.6, 20.6, 14.8; IR (neat) 3060, 3029, 2982, 2935, 2877, 1595, 1493, 1457, 1385, 1338, 1303, 1212, 1183, 1153, 1104, 1050, 971, 909, 887, 815, 752, 708, 668, 646, 625, 599, 573, 545, 517, 501, 465, 437, $419 \mathrm{~cm}^{-1}$; HRMS (FAB); Exact mass calcd for $\mathrm{C}_{30} \mathrm{H}_{37} \mathrm{~N}_{2} \mathrm{O}_{5} \mathrm{~S}_{2}[\mathrm{M}+\mathrm{H}]^{+}, 569.2144$. Found 569.2150. iso- 


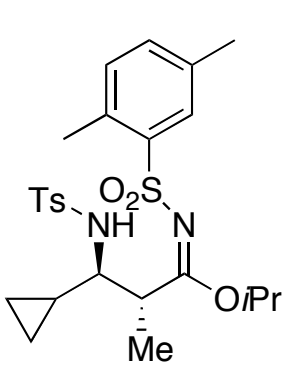

Propyl

anti-2-methyl-3-(cyclo-propyl)-3-(tertbutoxycarbonylamino $)-N-(2,5$-xylylsulfonyl)propionimidate (anti-7q): Mp. $97-100{ }^{\circ} \mathrm{C} ;{ }^{1} \mathrm{H} \mathrm{NMR}\left(\mathrm{CDCl}_{3}\right) ; \delta=8.14(\mathrm{~s}, 1 \mathrm{H})$, $7.76(\mathrm{~d}, 2 \mathrm{H}, J=8.5 \mathrm{~Hz}), 6.85(\mathrm{~d}, 1 \mathrm{H}, J=8.0 \mathrm{~Hz}), 6.79(\mathrm{~d}, 1 \mathrm{H}, J$ $=7.4 \mathrm{~Hz}), 6.73(\mathrm{~d}, 2 \mathrm{H}, J=8.5 \mathrm{~Hz}), 6.18(\mathrm{~d}, 1 \mathrm{H}, J=8.5 \mathrm{~Hz})$, 4.84 (quint, $1 \mathrm{H}, J=6.2 \mathrm{~Hz}$ ), $4.16(\mathrm{qd}, 1 \mathrm{H}, J=9.6,6.8 \mathrm{~Hz}), 3.25$ $(\mathrm{q}, 1 \mathrm{H}, J=9.0 \mathrm{~Hz}), 2.84(\mathrm{~s}, 3 \mathrm{H}), 1.97(\mathrm{~s}, 3 \mathrm{H}), 1.89(\mathrm{~s}, 3 \mathrm{H}), 1.18(\mathrm{~d}, 3 \mathrm{H}, J=6.2 \mathrm{~Hz})$, $1.16(\mathrm{~d}, 3 \mathrm{H}, J=6.2 \mathrm{~Hz}), 0.88(\mathrm{~d}, 3 \mathrm{H}, J=6.2 \mathrm{~Hz}), 0.27-0.37(\mathrm{~m}, 1 \mathrm{H}),(-) 0.10-0.10(\mathrm{~m}$, $3 \mathrm{H}),(-) 0.25-(-) 0.13(\mathrm{~m}, 1 \mathrm{H}) ;{ }^{13} \mathrm{C} \mathrm{NMR}\left(\mathrm{CDCl}_{3}\right) \delta=176.8,142.1,141.4,140.4,136.0$, 134.9, 133.4, 132.4, 129.3, 128.9, 127.1, 72.8, 61.4, 46.7, 21.4, 21.1, 21.0, 20.6, 15.9, 15.5, 4.8, 2.7; IR (neat) 3063, 2982, 2936, 2881, 1594, 1493, 1456, 1385, 1357, 1332, 1302, 1239, 1158, 1101, 1052, 1027, 981, 962, 909, 887, 816, 748, 709, 665, 645, 627, 601, 569, 548, 501, 464, 442,419 $\mathrm{cm}^{-1}$; HRMS (FAB); Exact mass calcd for $\mathrm{C}_{25} \mathrm{H}_{35} \mathrm{~N}_{2} \mathrm{O}_{5} \mathrm{~S}_{2}[\mathrm{M}+\mathrm{H}]^{+}$, 507.1987. Found 507.2003.

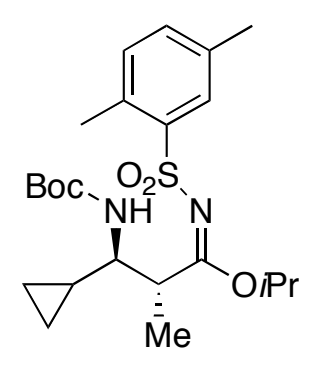

iso-Propyl anti-2-methyl-3-(cyclo-propyl)-3-(ptoluenesulfonylamino $)-N$-(2,5-xylylsulfonyl)propionimidate (anti-7r): ${ }^{1} \mathrm{H} \mathrm{NMR}\left(\mathrm{CDCl}_{3}\right) ; \delta=7.86(\mathrm{~s}, 1 \mathrm{H}), 7.27(\mathrm{~d}, 1 \mathrm{H}, J=$ $9.2 \mathrm{~Hz}), 7.20$ (d, 1H, $J=7.8 \mathrm{~Hz}), 5.28(\mathrm{~d}, 1 \mathrm{H}, J=10.1 \mathrm{~Hz}), 4.93-$ $5.09(\mathrm{~m}, 1 \mathrm{H}), 3.74(\mathrm{dq}, 1 \mathrm{H}, J=6.9,10.6 \mathrm{~Hz}), 3.44(\mathrm{dt}, 1 \mathrm{H}, J=$ 10.1, $10.1 \mathrm{~Hz}), 2.64(\mathrm{~s}, 3 \mathrm{H}), 2.38(\mathrm{~s}, 3 \mathrm{H}), 1.38(\mathrm{~s}, 9 \mathrm{H}), 1.34$ (d, $3 \mathrm{H}, J=6.9 \mathrm{~Hz}), 1.22(\mathrm{~d}, 3 \mathrm{H}, J=6.0 \mathrm{~Hz}), 1.19(\mathrm{~d}, 3 \mathrm{H}, J=6.4 \mathrm{~Hz}), 0.76-0.90(\mathrm{~m}, 1 \mathrm{H})$, 0.52-0.66 (m, 1H), 0.30-0.45 (m, 3H); ${ }^{13} \mathrm{C} \mathrm{NMR}\left(\mathrm{CDCl}_{3}\right) \delta=177.6,155.2,139.5$, 135.6, 134.1, 133.2, 132.1, 128.1, 76.8, 71.9, 56.3, 45.5, 28.2, 21.1, 20.8, 20.8, 20.0, 15.1, 15.0, 4.4, 0.0; IR (neat) 3386, 3082, 2979, 2935, 2882, 1715, 1590, 1513, 1457, 1390, 1365, 1299, 1252, 1229, 1156, 1103, 1057, 1023, 1001, 957, 747, 723, 707, 644, 600, 559, 533, $499 \mathrm{~cm}^{-1}$; HRMS (FAB); Exact mass calcd for $\mathrm{C}_{23} \mathrm{H}_{37} \mathrm{~N}_{2} \mathrm{O}_{5} \mathrm{~S}[\mathrm{M}+\mathrm{H}]^{+}$, 453.2423. Found 453.2434.

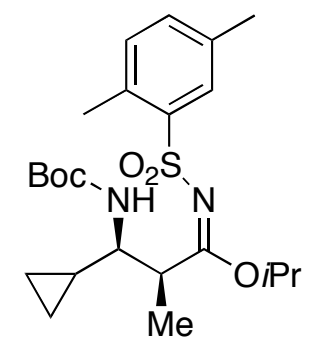

iso-Propyl

syn-2-methyl-3-(cyclo-propyl)-3-(ptoluenesulfonylamino)- $N$-(2,5-xylylsulfonyl)propionimidate (syn-7r): ${ }^{1} \mathrm{H} \mathrm{NMR}\left(\mathrm{CDCl}_{3}\right) ; \delta=7.85(\mathrm{~s}, 1 \mathrm{H}), 7.24(\mathrm{~d}, 1 \mathrm{H}, J=7.8$ $\mathrm{Hz}), 7.18(\mathrm{~d}, 1 \mathrm{H}, J=7.8 \mathrm{~Hz}), 4.98-5.10(\mathrm{~m}, 1 \mathrm{H}), 4.54(\mathrm{~d}, 1 \mathrm{H}, J=$ $9.6 \mathrm{~Hz}), 3.66-3.80(\mathrm{~m}, 1 \mathrm{H}), 3.29(\mathrm{q}, 1 \mathrm{H}, J=9.5 \mathrm{~Hz}), 2.62(\mathrm{~s}, 3 \mathrm{H})$, $2.37(\mathrm{~s}, 3 \mathrm{H}), 1.45$ (s, 9H), 1.26 (d, 3H, $J=6.0 \mathrm{~Hz}), 1.25$ (d, 3H, $J$ $=6.8 \mathrm{~Hz}), 1.21(\mathrm{~d}, 3 \mathrm{H}, J=6.4 \mathrm{~Hz}), 0.91-1.02(\mathrm{~m}, 1 \mathrm{H}), 0.26-0.56(\mathrm{~m}, 4 \mathrm{H}) ;{ }^{13} \mathrm{C} \mathrm{NMR}$ $\left(\mathrm{CDCl}_{3}\right) \delta=176.4,155.9,139.8,135.6,134.1,133.1,132.0,128.1,79.3,71.9,56.8$, $45.8,28.3,21.3,21.2,20.8,19.8,15.6,15.3,5.7,2.2$; IR (neat) 3366, 2979, 2933, 
1698, 1591, 1519, 1455, 1365, 1286, 1155, 1103, 1060, 1018, 956, 910, 824, 748, 706, 644, $500 \mathrm{~cm}^{-1}$; HRMS (FAB); Exact mass calcd for $\mathrm{C}_{23} \mathrm{H}_{37} \mathrm{~N}_{2} \mathrm{O}_{5} \mathrm{~S}[\mathrm{M}+\mathrm{H}]^{+}$, 453.2423. Found 453.2400.

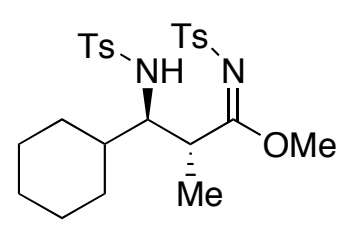

Methyl anti-2-methyl-3-(cyclo-hexyl)-3-( $p$ toluenesulfonylamino)- $N$-( $p$-toluenesulfonyl)propionimidate (anti-7s): ${ }^{1} \mathrm{H} \mathrm{NMR}\left(\mathrm{CDCl}_{3}\right) ; \delta=7.86$ (apparent $\mathrm{d}, 2 \mathrm{H}, J=8.5$ $\mathrm{Hz}), 7.70$ (d, 2H, $J=7.9 \mathrm{~Hz}), 7.32$ (d, 2H, $J=7.9 \mathrm{~Hz}), 7.19$ (d, $2 \mathrm{H}, J=7.9 \mathrm{~Hz}), 5.60(\mathrm{~d}, 1 \mathrm{H}, J=9.6 \mathrm{~Hz}), 3.87(\mathrm{dq}, 1 \mathrm{H}, J=6.2,10.2 \mathrm{~Hz}), 3.56(\mathrm{dt}, 1 \mathrm{H}$, $J=2.3,9.6 \mathrm{~Hz}), 3.50(\mathrm{~s}, 3 \mathrm{H}), 2.44(\mathrm{~s}, 3 \mathrm{H}), 2.37(\mathrm{~s}, 3 \mathrm{H}), 1.72-1.80(\mathrm{~m}, 1 \mathrm{H}), 1.54-1.68$ $(\mathrm{m}, 3 \mathrm{H}), 1.36-1.52(\mathrm{~m}, 2 \mathrm{H}), 1.20(\mathrm{~d}, 3 \mathrm{H}, J=6.8 \mathrm{~Hz}), 1.08-1.18(\mathrm{~m}, 2 \mathrm{H}), 0.84(\mathrm{~m}$, $3 \mathrm{H}) ;{ }^{13} \mathrm{C} \mathrm{NMR}\left(\mathrm{CDCl}_{3}\right) \delta=177.5,143.3,142.6,139.6,129.3,129.2,126.6,61.9$, 55.5, 41.7, 39.8, 30.9, 26.3, 26.1, 25.4, 21.5, 21.4, 15.5; IR (neat) 3306, 2928, 2854 , $1600,1495,1447,1380,1329,1303,1289,1182,1158,1091,1039,1011,951,913$, 887, 862, 814, 735, 706, 689, 596, 567, $547 \mathrm{~cm}^{-1}$; HRMS (FAB); Exact mass calcd for $\mathrm{C}_{25} \mathrm{H}_{35} \mathrm{~N}_{2} \mathrm{O}_{5} \mathrm{~S}_{2}[\mathrm{M}+\mathrm{H}]^{+}$, 507.1987. Found 507.1970.

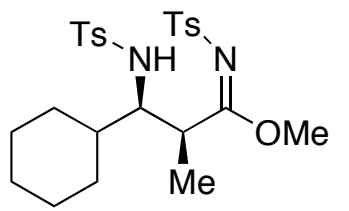

\section{Methyl syn-2-methyl-3-(cyclo-hexyl)-3-(p-} toluenesulfonylamino $)-N$-( $p$-toluenesulfonyl $)$ propionimidate (syn-7s): ${ }^{1} \mathrm{H} \mathrm{NMR}\left(\mathrm{CDCl}_{3}\right) ; \delta=7.85(\mathrm{~d}, 2 \mathrm{H}, J=8.2 \mathrm{~Hz}), 7.73$ $(\mathrm{d}, 2 \mathrm{H}, J=8.2 \mathrm{~Hz}), 7.30(\mathrm{~d}, 2 \mathrm{H}, J=8.2 \mathrm{~Hz}), 7.27(\mathrm{~d}, 2 \mathrm{H}, J=$ $8.2 \mathrm{~Hz}), 4.43(\mathrm{~d}, 1 \mathrm{H}, J=9.6 \mathrm{~Hz}), 3.79-3.85(\mathrm{~m}, 1 \mathrm{H}), 3.67-3.73(\mathrm{~m}, 4 \mathrm{H}), 2.43(\mathrm{~s}, 3 \mathrm{H})$, $2.41(\mathrm{~s}, 3 \mathrm{H}), 1.46-1.70(\mathrm{~m}, 5 \mathrm{H}), 0.93-1.20(\mathrm{~m}, 8 \mathrm{H}), 0.55-0.65(\mathrm{~m}, 1 \mathrm{H}) ;{ }^{13} \mathrm{C} \mathrm{NMR}$ $\left(\mathrm{CDCl}_{3}\right) \delta=176.6,143.1,143.1,139.2,138.7,129.4,129.3,127.0,126.6,60.3,55.7$, 41.2, 40.9, 30.3, 27.6, 26.2, 26.0, 25.9, 21.5, 21.5, 14.2, 13.5; IR (neat) 3293, 2928, 2854, 1599, 1496, 1448, 1319, 1300, 1185, 1157, 862, 814, 737, 687, 606, 570, 553 $\mathrm{cm}^{-1}$; HRMS (FAB); Exact mass calcd for $\mathrm{C}_{25} \mathrm{H}_{35} \mathrm{~N}_{2} \mathrm{O}_{5} \mathrm{~S}_{2}[\mathrm{M}+\mathrm{H}]^{+}, 507.1987$. Found 507.1970.

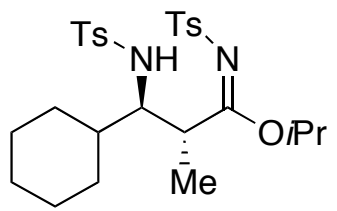

iso-Propyl anti-2-methyl-3-(cyclo-hexyl)-3-(ptoluenesulfonylamino)- $N$-(p-toluenesulfonyl)propionimidate (anti-7t): ${ }^{1} \mathrm{H} \mathrm{NMR}\left(\mathrm{CDCl}_{3}\right) ; \delta=7.85(\mathrm{~d}, 2 \mathrm{H}, J=8.2 \mathrm{~Hz}), 7,71$ $(\mathrm{d}, 2 \mathrm{H}, J=8.2 \mathrm{~Hz}), 7.30(\mathrm{~d}, 2 \mathrm{H}, J=8.2 \mathrm{~Hz}), 7.20(\mathrm{~d}, 2 \mathrm{H}, J=$ $7.8 \mathrm{~Hz}), 5.68(\mathrm{~d}, 1 \mathrm{H}, J=9.6 \mathrm{~Hz}), 4.76-4.88(\mathrm{~m}, 1 \mathrm{H}), 3.84(\mathrm{dq}, 1 \mathrm{H}, J=6.9,10.0 \mathrm{~Hz})$, $3.59(\mathrm{dt}, 1 \mathrm{H}, J=2.7,9.2 \mathrm{~Hz}), 2.43(\mathrm{~s}, 3 \mathrm{H}), 2.37(\mathrm{~s}, 3 \mathrm{H}), 0.75-1.75(\mathrm{~m}, 11 \mathrm{H}), 1.30(\mathrm{~d}$, $3 \mathrm{H}, J=6.4 \mathrm{~Hz}), 1.22(\mathrm{~d}, 3 \mathrm{H}, J=6.0 \mathrm{~Hz}), 1.17(\mathrm{~d}, 3 \mathrm{H}, J=6.4 \mathrm{~Hz}) ;{ }^{13} \mathrm{C} \mathrm{NMR}\left(\mathrm{CDCl}_{3}\right)$ $\delta=176.4,143.1,142.5,139.8,129.3,129.1,126.5,126.5,73.1,61.9,41.8,39.8,30.8$, 
26.3, 26.1, 25.8, 21.5, 21.4, 21.2, 20.8, 15.7; IR (neat) 3277, 2982, 2929, 2854, 2308, 1593, 1495, 1449, 1385, 1374, 1331, 1302, 1182, 1158, 1091, 1020, 968, 909, 887, 862, 840, 814, 735, 694, 671, 605, 582, $547 \mathrm{~cm}^{-1}$; HRMS (FAB); Exact mass calcd for $\mathrm{C}_{27} \mathrm{H}_{39} \mathrm{~N}_{2} \mathrm{O}_{5} \mathrm{~S}_{2}[\mathrm{M}+\mathrm{H}]^{+}$, 535.2300. Found 535.2297.

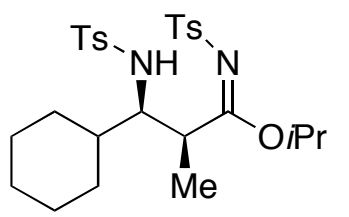

\section{iso-Propyl syn-2-methyl-3-(cyclo-hexyl)-3-(p-} toluenesulfonylamino)- $N$-( $p$-toluenesulfonyl)propionimidate (syn-7t): ${ }^{1} \mathrm{H} \mathrm{NMR}\left(\mathrm{CDCl}_{3}\right) ; \delta=7.81(\mathrm{~d}, 2 \mathrm{H}, J=8.2 \mathrm{~Hz}), 7.75$ $(\mathrm{d}, 2 \mathrm{H}, J=8.2 \mathrm{~Hz}), 7.25-7.33(\mathrm{~m}, 4 \mathrm{H}), 4.97-5.08(\mathrm{~m}, 1 \mathrm{H}), 4.39$ $(\mathrm{d}, 1 \mathrm{H}, J=8.7 \mathrm{~Hz}), 3.71(\mathrm{dq}, 1 \mathrm{H}, J=6.4,9.2 \mathrm{~Hz}), 3.62(\mathrm{dt}, 1 \mathrm{H}, J=3.2,9.2 \mathrm{~Hz}), 2.42$ $(\mathrm{s}, 6 \mathrm{H}), 0.65-1.71(\mathrm{~m}, 11 \mathrm{H}), 1.25(\mathrm{~d}, 3 \mathrm{H}, J=6.4 \mathrm{~Hz}), 1.22(\mathrm{~d}, 3 \mathrm{H}, J=6.4 \mathrm{~Hz}), 1.06$ (d, $3 \mathrm{H}, J=6.9 \mathrm{~Hz}) ;{ }^{13} \mathrm{C} \mathrm{NMR}\left(\mathrm{CDCl}_{3}\right) \delta=175.5,143.1,143.0,139.3,138.9,129.4$, $129.3,127.0,126.5,72.3,60.8,41.6,41.4,31.0,26.6,26.4,26.1,26.0,21.5,21.5$, 21.0, 20.9, 15.1; IR (neat) 3295, 2981, 2928, 2854, 1596, 1496, 1449, 1381, 1300, 1184, 1159, 1093, 1053, 1019, 982, 960, 909, 839, 814, 718, 693, 609, 569, $547 \mathrm{~cm}^{-1}$; HRMS (FAB); Exact mass calcd for $\mathrm{C}_{27} \mathrm{H}_{39} \mathrm{~N}_{2} \mathrm{O}_{5} \mathrm{~S}_{2}[\mathrm{M}+\mathrm{H}]^{+}, 535.2300$. Found 535.2297.

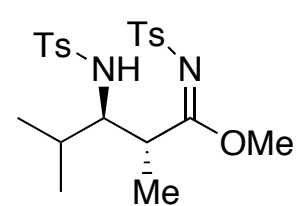

Methyl anti-2-methyl-3-(iso-propyl)-3-(ptoluenesulfonylamino $)-N$-( $p$-toluenesulfonyl)propionimidate (anti-7u): Mp. $147-148{ }^{\circ} \mathrm{C} ;{ }^{1} \mathrm{H} \mathrm{NMR}\left(\mathrm{CDCl}_{3}\right) ; \delta=7.88(\mathrm{~d}, 2 \mathrm{H}, J$ $=8.5 \mathrm{~Hz}), 7.71(\mathrm{~d}, 2 \mathrm{H}, J=8.5 \mathrm{~Hz}), 7.32(\mathrm{~d}, 2 \mathrm{H}, J=7.9 \mathrm{~Hz}), 7.19$ $(\mathrm{d}, 2 \mathrm{H}, J=7.9 \mathrm{~Hz}), 5.63(\mathrm{~d}, 1 \mathrm{H}, J=9.6 \mathrm{~Hz}), 3.82(\mathrm{dq}, 1 \mathrm{H}, J=6.8,10.2 \mathrm{~Hz}), 3.62(\mathrm{dt}$, $1 \mathrm{H}, J=2.8,10.2 \mathrm{~Hz}), 3.57$ (s, 3H), 2.44 (s, 3H), 2.37 (s, 3H), 1.83-1.93 (m, 1H), 1.21 $(\mathrm{d}, 3 \mathrm{H}, J=6.2 \mathrm{~Hz}), 0.83(\mathrm{~d}, 3 \mathrm{H}, J=6.8 \mathrm{~Hz}), 0.71(\mathrm{~d}, 3 \mathrm{H}, J=6.8 \mathrm{~Hz}) ;{ }^{13} \mathrm{C} \mathrm{NMR}$ $\left(\mathrm{CDCl}_{3}\right) \delta=177.4,143.3,142.6,139.6,138.7,129.3,129.2,126.7,126.6,62.2,55.6$, 42.5, 28.9, 21.5, 21.4, 20.6, 15.3, 14.8; IR (neat) 3307, 2965, 1601, 1540, 1496, 1455 , 1329, 1304, 1287, 1157, 1090, 1044, 950, 814, 734, 688, 666, 596, 584, $547 \mathrm{~cm}^{-1}$; HRMS (FAB); Exact mass calcd for $\mathrm{C}_{22} \mathrm{H}_{31} \mathrm{~N}_{2} \mathrm{O}_{5} \mathrm{~S}_{2}[\mathrm{M}+\mathrm{H}]^{+}, 467.1674$. Found 467.1673.

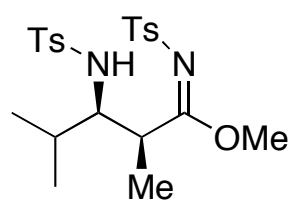

Methyl syn-2-methyl-3-( iso-propyl)-3-(ptoluenesulfonylamino)- $N$-( $p$-toluenesulfonyl)propionimidate (syn-7u): ${ }^{1} \mathrm{H} \mathrm{NMR}\left(\mathrm{CDCl}_{3}\right) ; \delta=7.83(\mathrm{~d}, 2 \mathrm{H}, J=8.2 \mathrm{~Hz}), 7.73(\mathrm{~d}$, $2 \mathrm{H}, J=8.2 \mathrm{~Hz}), 7.29(\mathrm{~d}, 2 \mathrm{H}, J=8.2 \mathrm{~Hz}), 7.25(\mathrm{~d}, 2 \mathrm{H}, J=8.2 \mathrm{~Hz})$, $4.51(\mathrm{~d}, 1 \mathrm{H}, J=10.1 \mathrm{~Hz}), 3.71(\mathrm{~s}, 3 \mathrm{H}), 3.66-3.80(\mathrm{~m}, 2 \mathrm{H}), 2.41(\mathrm{~s}, 3 \mathrm{H}), 2.39(\mathrm{~s}, 3 \mathrm{H})$, 1.49-1.63 (m, 1H), $1.13(\mathrm{~d}, 3 \mathrm{H}, J=6.9 \mathrm{~Hz}), 0.76(\mathrm{~d}, 3 \mathrm{H}, J=6.9 \mathrm{~Hz}), 0.71(\mathrm{~d}, 3 \mathrm{H}, J=$ 
$6.9 \mathrm{~Hz}) ;{ }^{13} \mathrm{C} \mathrm{NMR}\left(\mathrm{CDCl}_{3}\right) \delta=176.6,143.2,143.1,139.1,138.8,129.5,129.4,126.9$, 126.5, 60.9, 55.6, 41.8, 31.2, 21.5, 21.5, 20.4, 16.7, 14.2; IR (neat) 3295, 2965, 2882 , 1599, 1495, 1455, 1436, 1325, 1290, 1157, 1092, 1039, 952, 909, 814, 688, 666, 603, $573,548 \mathrm{~cm}^{-1}$; HRMS (FAB); Exact mass calcd for $\mathrm{C}_{22} \mathrm{H}_{31} \mathrm{~N}_{2} \mathrm{O}_{5} \mathrm{~S}_{2}[\mathrm{M}+\mathrm{H}]^{+}$, 467.1674. Found 467.1682.

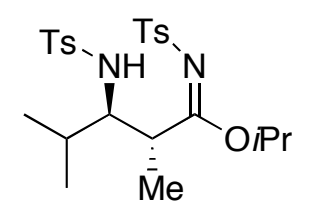

iso-Propyl anti-2-methyl-3-( iso-propyl)-3-(ptoluenesulfonylamino $)-\boldsymbol{N}$-( $p$-toluenesulfonyl)propionimidate (anti-7v): ${ }^{1} \mathrm{H} \mathrm{NMR}\left(\mathrm{CDCl}_{3}\right) ; \delta=7.87(\mathrm{~d}, 2 \mathrm{H}, J=8.2 \mathrm{~Hz}), 7.72(\mathrm{~d}$, $2 \mathrm{H}, J=8.2 \mathrm{~Hz}), 7.31(\mathrm{~d}, 2 \mathrm{H}, J=7.7 \mathrm{~Hz}), 7.20(\mathrm{~d}, 2 \mathrm{H}, J=8.2 \mathrm{~Hz})$, $5.74(\mathrm{~d}, 1 \mathrm{H}, J=9.1 \mathrm{~Hz}), 4.83-4.91(\mathrm{~m}, 1 \mathrm{H}), 3.79(\mathrm{dq}, 1 \mathrm{H}, J=6.3,10.0 \mathrm{~Hz}), 3.67(\mathrm{dt}$, $1 \mathrm{H}, J=2.3,9.0 \mathrm{~Hz}), 2.43(\mathrm{~s}, 3 \mathrm{H}), 2.38(\mathrm{~s}, 3 \mathrm{H}), 1.80-1.90(\mathrm{~m}, 1 \mathrm{H}), 1.33(\mathrm{~d}, 3 \mathrm{H}, J=6.3$ $\mathrm{Hz}), 1.23(\mathrm{~d}, 3 \mathrm{H}, J=6.3 \mathrm{~Hz}), 1.19(\mathrm{~d}, 3 \mathrm{H}, J=6.8 \mathrm{~Hz}), 0.80(\mathrm{~d}, 3 \mathrm{H}, J=6.8 \mathrm{~Hz}), 0.69$ $(\mathrm{d}, 3 \mathrm{H}, J=6.8 \mathrm{~Hz}) ;{ }^{13} \mathrm{C} \mathrm{NMR}\left(\mathrm{CDCl}_{3}\right) \delta=176.4,143.1,142.5,139.9,139.0,129.3$, 129.2, 126.5, 126.5, 73.2, 62.3, 42.6, 29.2, 21.5, 21.4, 21.2, 20.9, 20.6, 15.6, 14.9; IR (neat) $3647,3273,2968,2936,2877,1918,1736,1592,1496,1455,1387,1373,1331$, 1286, 1241, 1184, 1155, 1089, 1042, 968, 909, 883, 849, 814, 727, 694, 666, 609, 579, $547 \mathrm{~cm}^{-1}$; HRMS (FAB); Exact mass calcd for $\mathrm{C}_{24} \mathrm{H}_{35} \mathrm{~N}_{2} \mathrm{O}_{5} \mathrm{~S}_{2}[\mathrm{M}+\mathrm{H}]^{+}, 495.1987$. Found 495.1969.

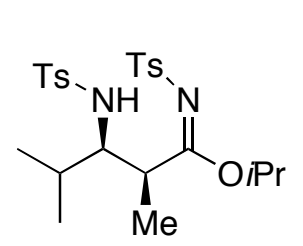

iso-Propyl syn-2-methyl-3-( iso-propyl)-3-(ptoluenesulfonylamino $)-N$-( $p$-toluenesulfonyl)propionimidate (syn-7v): ${ }^{1} \mathrm{H} \mathrm{NMR}\left(\mathrm{CDCl}_{3}\right) ; \delta=7.80(\mathrm{~d}, 2 \mathrm{H}, J=8.5 \mathrm{~Hz}), 7.75(\mathrm{~d}$, $2 \mathrm{H}, J=7.9 \mathrm{~Hz}), 7.26-7.31(\mathrm{~m}, 4 \mathrm{H}), 4.96-5.06(\mathrm{~m}, 1 \mathrm{H}), 4.35-4.42$ $(\mathrm{m}, 1 \mathrm{H}), 3.62-3.71(\mathrm{~m}, 2 \mathrm{H}), 2.42(\mathrm{~s}, 3 \mathrm{H}), 2.41(\mathrm{~s}, 3 \mathrm{H}), 1.55-1.65(\mathrm{~m}, 1 \mathrm{H}), 1.25(\mathrm{~d}, 3 \mathrm{H}$, $J=6.2 \mathrm{~Hz}), 1.22(\mathrm{~d}, 3 \mathrm{H}, J=6.2 \mathrm{~Hz}), 1.11(\mathrm{~d}, 3 \mathrm{H}, J=6.2 \mathrm{~Hz}), 0.79(\mathrm{~d}, 3 \mathrm{H}, J=6.8$ $\mathrm{Hz}), 0.73(\mathrm{~d}, 3 \mathrm{H}, J=6.8 \mathrm{~Hz}) ;{ }^{13} \mathrm{C} \mathrm{NMR}\left(\mathrm{CDCl}_{3}\right) \delta=175.4,143.1,143.1,139.3$, 139.0, 129.5, 129.3, 126.9, 126.5, 72.3, 61.1, 42.4, 31.0, 21.5, 21.5, 21.0, 20.9, 20.8, 15.9, 15.5; IR (neat) 3295, 2977, 2933, 2878, 1594, 1456, 1374, 1317, 1290, 1159, 1092, 1040, 909, 839, 814, 718, 693, 666, 608, 572, 548, $458 \mathrm{~cm}^{-1}$; HRMS (FAB); Exact mass calcd for $\mathrm{C}_{24} \mathrm{H}_{35} \mathrm{~N}_{2} \mathrm{O}_{5} \mathrm{~S}_{2}[\mathrm{M}+\mathrm{H}]^{+}$, 495.1987. Found 495.1969.

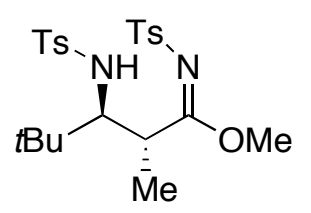

Methyl anti-3-(p-toluenesulfonylamino)-2,4,4-trimethyl- $N$ - $(p$ toluenesulfonyl)pentanimidate (anti-7w): ${ }^{1} \mathrm{H} \mathrm{NMR}\left(\mathrm{CDCl}_{3}\right) ; \delta=$ $7.83(\mathrm{~d}, 2 \mathrm{H}, J=8.2 \mathrm{~Hz}), 7.73(\mathrm{~d}, 2 \mathrm{H}, J=8.2 \mathrm{~Hz}), 7.31(\mathrm{~d}, 2 \mathrm{H}, J=$ $8.2 \mathrm{~Hz}), 7.25(\mathrm{~d}, 2 \mathrm{H}, J=8.2 \mathrm{~Hz}), 5.47(\mathrm{~d}, 1 \mathrm{H}, J=9.6 \mathrm{~Hz}), 4.03(\mathrm{dq}, 1 \mathrm{H}, J=6.8,6.9$ $\mathrm{Hz}), 3.68$ (s, 3H), 3.43 (dd, 1H, J = 5.5, $10.4 \mathrm{~Hz}), 2.43$ (s, 3H), 2.40 (s, 3H), 1.25 (d, 
$3 \mathrm{H}, J=6.9 \mathrm{~Hz}), 0.87(\mathrm{~s}, 9 \mathrm{H}) ;{ }^{13} \mathrm{C} \mathrm{NMR}\left(\mathrm{CDCl}_{3}\right) \delta=177.4,143.4,142.8,139.6$, 138.8, 129.4, 126.6, 66.6, 55.5, 39.4, 36.4, 27.5, 21.5, 21.5, 18.4; IR (neat) 3325, 2954, 1601, 1540, 1455, 1315, 1259, 1185, 1157, 1091, 1025, 927, 814, 675, 594, 572, $547 \mathrm{~cm}^{-1}$; HRMS (FAB); Exact mass calcd for $\mathrm{C}_{23} \mathrm{H}_{34} \mathrm{~N}_{3} \mathrm{O}_{5} \mathrm{~S}_{2}[\mathrm{M}+\mathrm{H}]^{+}, 481.1831$. Found 481.1832 .

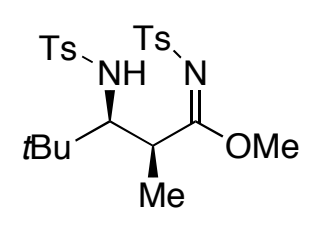

Methyl syn-3-(p-toluenesulfonylamino)-2,4,4-trimethyl- $N-(p-$ toluenesulfonyl)pentanimidate (syn-7w): Mp. $189-190{ }^{\circ} \mathrm{C} ;{ }^{1} \mathrm{H}$ $\operatorname{NMR}\left(\mathrm{CDCl}_{3}\right) ; \delta=7.84(\mathrm{~d}, 2 \mathrm{H}, J=8.2 \mathrm{~Hz}), 7.74(\mathrm{~d}, 2 \mathrm{H}, J=8.2$ $\mathrm{Hz}), 7.30(\mathrm{~d}, 2 \mathrm{H}, J=8.2 \mathrm{~Hz}), 7.26(\mathrm{~d}, 2 \mathrm{H}, J=8.2 \mathrm{~Hz}), 4.65-4.72$ (m, 1H), 3.80-3.88 (m, 2H), $3.71(\mathrm{~s}, 3 \mathrm{H}), 2.42(\mathrm{~s}, 3 \mathrm{H}), 2.40(\mathrm{~s}, 3 \mathrm{H}), 1.18(\mathrm{~d}, 3 \mathrm{H}, J=$ $6.2 \mathrm{~Hz}), 0.82(\mathrm{~s}, 9 \mathrm{H}) ;{ }^{13} \mathrm{C} \mathrm{NMR}\left(\mathrm{CDCl}_{3}\right) \delta=177.7,143.1$ 142.9, 139.2, 139.1, 129.4, 129.3, 126.8, 126.5, 63.1, 55.4, 40.0, 36.4, 26.8, 21.5, 21.4, 16.4; IR (neat) 3303, 2952, 1600, 1540, 1456, 1436, 1315, 1301, 1286, 1155, 1093, 1071, 953, 916, 814, $688,605,574 \mathrm{~d} \mathrm{~cm}^{-1}$; HRMS (FAB); Exact mass calcd for $\mathrm{C}_{23} \mathrm{H}_{34} \mathrm{~N}_{3} \mathrm{O}_{5} \mathrm{~S}_{2}[\mathrm{M}+\mathrm{H}]^{+}$, 481.1831. Found 481.1832.

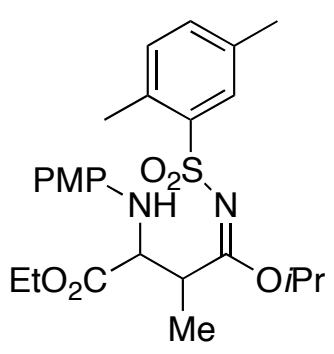

iso-Propyl 3-ethoxycarbonyl-3-(p-methoxyphenylamino)-2methyl- $N$-(2,5-xylylsulfonyl)propionimidate (major-7x): Mp. 99-100 ${ }^{\circ} \mathrm{C} ;{ }^{1} \mathrm{H}$ NMR $\left(\mathrm{CDCl}_{3}\right) ; \delta=8.04-8.09$ (m, 2H), 6.95-7.02 $(\mathrm{m}, 3 \mathrm{H}), 6.67-6.74(\mathrm{~m}, 4 \mathrm{H}), 4.90(\mathrm{~d}, 1 \mathrm{H}, J=11.9 \mathrm{~Hz}), 4.56(\mathrm{qd}$, $1 \mathrm{H}, J=10.2,6.8 \mathrm{~Hz}), 4.37(\mathrm{t}, 1 \mathrm{H}, J=10.8 \mathrm{~Hz}), 3.78-3.93(\mathrm{~m}$, 2H), 3.29 (s, 3H), $3.08(\mathrm{~s}, 3 \mathrm{H}), 1.29(\mathrm{~d}, 3 \mathrm{H}, J=6.2 \mathrm{~Hz}), 0.82(\mathrm{t}$, $3 \mathrm{H}, J=7.1 \mathrm{~Hz}) ;{ }^{13} \mathrm{C} \mathrm{NMR}\left(\mathrm{CDCl}_{3}\right) \delta=176.5,171.8,153.9,142.8,141.0,132.4$, 128.8, 127.0, 116.2, 115.1, 62.1, 61.1, 55.1, 42.6, 14.4, 14.1; IR (neat) 3055, 2986, 2953, 2836, 1735, 1607, 1514, 1457, 1447, 1421, 1305, 1265, 1242, 1191, 1156, 1092, 1034, 953, 896, 825, 737, 705, 624, 603, 527, 451, $419 \mathrm{~cm}^{-1}$; HRMS (FAB); Exact mass calcd for $\mathrm{C}_{25} \mathrm{H}_{35} \mathrm{~N}_{2} \mathrm{O}_{6} \mathrm{~S}[\mathrm{M}+\mathrm{H}]^{+}$, 491.2216. Found 491.2198.

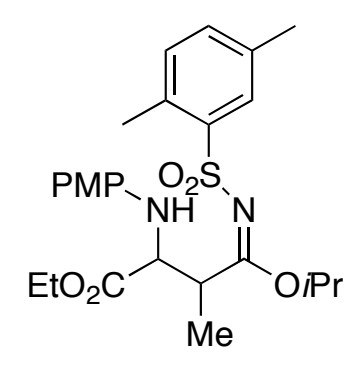

iso-Propyl 3-ethoxycarbonyl-3-(p-methoxyphenylamino)-2methyl- $N$-(2,5-xylylsulfonyl)propionimidate (minor-7x): ${ }^{1} \mathrm{H}$ $\operatorname{NMR}\left(\mathrm{CDCl}_{3}\right) ; \delta=8.05-8.14(\mathrm{~m}, 2 \mathrm{H}), 6.95-7.05(\mathrm{~m}, 3 \mathrm{H}), 6.74$ $(\mathrm{d}, 2 \mathrm{H}, J=9.1 \mathrm{~Hz}), 6.62(\mathrm{~d}, 2 \mathrm{H}, J=9.1 \mathrm{~Hz}), 4.50(\mathrm{t}, 1 \mathrm{H}, J=9.4$ $\mathrm{Hz}), 4.40(\mathrm{qd}, 1 \mathrm{H}, J=9.0,6.8 \mathrm{~Hz}), 3.92-4.07(\mathrm{~m}, 2 \mathrm{H}), 3.84(\mathrm{~d}$, $1 \mathrm{H}, J=10.2 \mathrm{~Hz}), 3.35(\mathrm{~s}, 3 \mathrm{H}), 3.21(\mathrm{~s}, 3 \mathrm{H}), 1.35$ (d, 3H, $J=8.0$ $\mathrm{Hz}), 0.94(\mathrm{t}, 3 \mathrm{H}, J=7.1 \mathrm{~Hz}) ;{ }^{13} \mathrm{C} \mathrm{NMR}\left(\mathrm{CDCl}_{3}\right) \delta=176.3,171.7,143.1,141.6,132.3$, $128.8,127.2,115.9,115.1,61.5,60.7,55.2,55.0,42.9,14.8,14.1$; IR (neat) 3063, 
3033, 2984, 2951, 2907, 2834, 1735, 1608, 1511, 1446, 1369, 1306, 1241, 1198, 1156, 1092, 1077, 1060, 1027, 953, 852, 824, 758, 734, 690, 624, 587, 519, 446, $418 \mathrm{~cm}^{-1}$; HRMS (FAB); Exact mass calcd for $\mathrm{C}_{25} \mathrm{H}_{35} \mathrm{~N}_{2} \mathrm{O}_{6} \mathrm{~S}[\mathrm{M}+\mathrm{H}]^{+}$, 491.2216. Found 491.2198 .

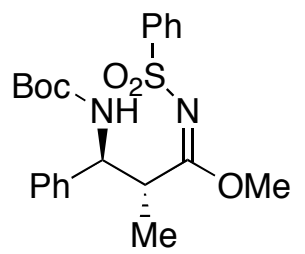

\section{Methyl anti- $N$-(benzenesulfonyl)-3-(tert-}

butoxycarbonylamino)-2-methyl-3-phenylpropionimidate (7y):

Mp. 99-100 ${ }^{\circ} \mathrm{C} ;{ }^{1} \mathrm{H}$ NMR $\left(\mathrm{CDCl}_{3}\right) \delta=8.01(\mathrm{~d}, 2 \mathrm{H}, J=7.4 \mathrm{~Hz})$, $7.59(\mathrm{t}, 1 \mathrm{H}, J=7.4 \mathrm{~Hz}), 7.53(\mathrm{t}, 2 \mathrm{H}, J=7.7 \mathrm{~Hz}), 7.25-7.35(\mathrm{~m}$,

$5 \mathrm{H}), 6.01(\mathrm{~d}, 1 \mathrm{H}, J=9.6 \mathrm{~Hz}), 4.75(\mathrm{t}, 1 \mathrm{H}, J=10.2 \mathrm{~Hz}), 3.96-4.07(\mathrm{~m}, 1 \mathrm{H}), 3.75(\mathrm{~s}$, $3 \mathrm{H}), 1.35(\mathrm{~s}, 9 \mathrm{H}), 1.03(\mathrm{~d}, 3 \mathrm{H}, J=6.8 \mathrm{~Hz}) ;{ }^{13} \mathrm{C} \mathrm{NMR}\left(\mathrm{CDCl}_{3}\right) \delta=177.6,154.6$, $141.8,140.2,132.6,128.7,128.7,127.8,127.1,126.5,79.2,58.2,55.5,45.1,28.2$, 14.8; IR (neat) 3381, 2977, 1716, 1602, 1508, 1455, 1392, 1365, 1304, 1246, 1154, 1090, 1057, 1002, 950, 881, 757, 734, 700, 688, $623 \mathrm{~cm}^{-1}$; HRMS (FAB); Exact mass calcd for $\mathrm{C}_{22} \mathrm{H}_{29} \mathrm{~N}_{2} \mathrm{O}_{5} \mathrm{~S}[\mathrm{M}+\mathrm{H}]^{+}, 433.1797$. Found 433.1811 .

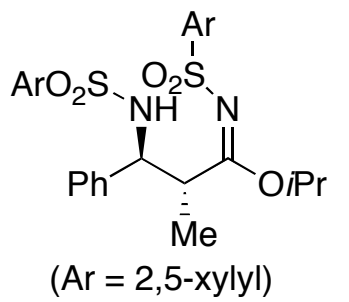

iso-Propyl anti-2-methyl-3-phenyl- $N$-(2,5-xylylsulfonyl)-3(2,5-xylylsulfonylamino)propionimidate (anti-7z): Mp. 99$100{ }^{\circ} \mathrm{C} ;{ }^{1} \mathrm{H}$ NMR $\left(\mathrm{CDCl}_{3}\right) ; \delta=8.23(\mathrm{~s}, 1 \mathrm{H}), 7.39(\mathrm{~s}, 1 \mathrm{H}), 7.12$ $(\mathrm{d}, 1 \mathrm{H}, J=9.1 \mathrm{~Hz}), 6.90-6.97(\mathrm{~m}, 3 \mathrm{H}), 6.87(\mathrm{dd}, 1 \mathrm{H}, J=7.6,1.4$ $\mathrm{Hz}), 6.65-6.73(\mathrm{~m}, 3 \mathrm{H}), 6.53(\mathrm{qd}, 2 \mathrm{H}, J=7.6,1.4 \mathrm{~Hz}), 5.03$ (quint, $1 \mathrm{H}, J=6.2 \mathrm{~Hz}$ ), $4.68(\mathrm{dd}, 1 \mathrm{H}, J=11.0,9.3 \mathrm{~Hz}), 4.48(\mathrm{qd}, 1 \mathrm{H}, J=11.0,6.5$ $\mathrm{Hz}), 3.00$ (s, 3H), 2.62 (s, 3H), 1.95 (s, 3H), 1.78 (s, 3H), 1.40 (d, 3H, J=6.2 Hz), $0.96(\mathrm{~d}, 3 \mathrm{H}, J=6.2 \mathrm{~Hz}), 0.91(\mathrm{~d}, 3 \mathrm{H}, J=6.2 \mathrm{~Hz}) ;{ }^{13} \mathrm{C} \mathrm{NMR}\left(\mathrm{CDCl}_{3}\right) \delta=177.0,140.1$, $140.0,138.0,136.1,135.2$, 135.0, 133.6, 133.2, 132.6, 132.2, 131.9, 129.7, 128.8, 128.3, 127.3, 73.1, 62.5, 46.4, 21.4, 21.1, 20.8, 20.5, 20.4, 20.0, 14.5; IR (neat) 3060, 3029, 2982, 2936, 2878, 1592, 1578, 1493, 1458, 1386, 1359, 1331, 1292, 1226, 1208, 1162, 1102, 1065, 1030, 973, 910, 855, 824, 768, 747, 725, 707, 645, 607, 580, 548, 516, 501, 464, 436, $419 \mathrm{~cm}^{-1}$; HRMS (FAB); Exact mass calcd for $\mathrm{C}_{29} \mathrm{H}_{37} \mathrm{~N}_{2} \mathrm{O}_{5} \mathrm{~S}_{2}$ $[\mathrm{M}+\mathrm{H}]^{+}$, 557.2144. Found 557.2151.

\section{Procedure of addition reaction of sulfonylimidate 6a to methyl acrylate. To MS} 4A (100 mg) were added a solution of sulfonylimidate $\mathbf{6 a}(68.1 \mathrm{mg}, 0.3 \mathrm{mmol})$ in DMF (0.5 mL), methyl acrylate $(38.7 \mathrm{mg}, 0.45 \mathrm{mmol})$, and a solution of DBU in DMF $(10 \mathrm{~mol} \%, 100 \mu \mathrm{l})$ at RT. The reaction mixture was stirred for $20 \mathrm{~h}$ at RT, and then diluted by addition of $\mathrm{Et}_{2} \mathrm{O}$. The mixture obtained after filtration (for removal of 
MS 4A) was washed with water 3 times, then dried over anhydrous $\mathrm{Na}_{2} \mathrm{SO}_{4}$. Filtration and removal of solvents afforded the crude product. Purification of the crude product was conducted by chromatography on $\mathrm{SiO}_{2}$, to afford the desired product $\mathbf{8}(70.4 \mathrm{mg}, 75 \%)$.<smiles>COC(=O)CCC(C)/C(=N\SO)OC</smiles>

Methyl N-(benzenesulfonyl)-4-methoxycarbonyl-2methylbutanimidate (8): ${ }^{1} \mathrm{H} \mathrm{NMR}\left(\mathrm{CDCl}_{3}\right) ; \delta=8.02-8.08(\mathrm{~m}$, 2H), 6.98-7.05 (m, 3H), 3.84-3.92 (m, 1H), 3.40 (s, 3H), 3.08 (s, 3H), 2.28-2.33 (m, 1H), 2.15-2.24 (m, 1H), 1.92-2.00 (m, $1 \mathrm{H}), 1.64-1.74(\mathrm{~m}, 1 \mathrm{H}), 1.35(\mathrm{~d}, 3 \mathrm{H}, J=6.8 \mathrm{~Hz}) ;{ }^{13} \mathrm{C} \mathrm{NMR}\left(\mathrm{CDCl}_{3}\right) \delta=178.5,172.6$, 143.2, 132.2, 128.8, 127.0, 54.7, 51.2, 38.8, 31.9, 29.3, 17.8; IR (neat) 3064, 2952, 2879, 2852, 1738, 1603, 1446, 1350, 1307, 1263, 1197, 1155, 1123, 1092, 1026, 977 , 951, 921, 839, 759, 734, 691, 626, 595, 536, 444, $419 \mathrm{~cm}^{-1}$; HRMS (FAB); Exact mass calcd for $\mathrm{C}_{14} \mathrm{H}_{20} \mathrm{NO}_{5} \mathrm{~S}[\mathrm{M}+\mathrm{H}]^{+}, 370.1683$. Found 370.1700 .

\section{Procedure of addition reaction of sulfonylimidate 6a to di-tert-butyl}

azodicarboxylate. To MS 4A (100 mg) were added a solution of sulfonylimidate $6 \mathbf{a}$ (136.4 mg, $0.6 \mathrm{mmol})$ in DMF (1.1 ml), di-tert-butyl azodicarboxylate (152 mg, 0.66 mmol), and a solution of DBU in DMF (5 mol\%, $100 \mu \mathrm{l})$ at RT. The reaction mixture was stirred for $1.5 \mathrm{~h}$ at $\mathrm{RT}$, and then diluted by addition of $\mathrm{Et}_{2} \mathrm{O}$. The mixture obtained after filtration (for removal of MS 4A) was washed with water 3 times, then dried over anhydrous $\mathrm{Na}_{2} \mathrm{SO}_{4}$. Filtration and removal of solvents afforded the crude product. Purification of the crude product was conducted by chromatography on $\mathrm{SiO}_{2}$, to afford the desired product $9(236.3 \mathrm{mg}, 86 \%)$.<smiles>[M]C(C(=O)OCC(C)C)N(NC(=O)OCC)/C(=N\Sc1ccccc1)OC</smiles>

\section{Methyl $N$-(benzenesulfonyl)-2-( $N, N^{\prime}$-bis-(tert-} butoxycarbonyl)-hydrazino)-2-methylbutanimidate (9): ${ }^{1} \mathrm{H}$ NMR (DMSO-d6, $\left.70{ }^{\circ} \mathrm{C}\right) \delta=8.16-8.80(\mathrm{~m}, 1 \mathrm{H}), 7.90-7.94$ (m, 2H), 7.53-7.69 (m, 3H), 5.50-5.63 (m, 1H), $3.70(\mathrm{~s}, 3 \mathrm{H})$, 1.35-1.45 (m, 21H); ${ }^{13} \mathrm{C}$ NMR (DMSO-d6, $\left.70{ }^{\circ} \mathrm{C}\right) \delta=141.6,132.3,128.7,125.7$, 79.3, 55.4, 55.3, 54.4, 27.7, 27.6, 14.6; IR (neat) 3326, 2979, 1732, 1613, 1540, 1507, 1478, 1448, 1393, 1368, 1307, 1247, 1155, 1092, 1075, 1024, 955, 911, 854, 758, 733, $690,611,585 \mathrm{~cm}^{-1}$; HRMS (FAB); Exact mass calcd for $\mathrm{C}_{20} \mathrm{H}_{32} \mathrm{~N}_{3} \mathrm{O}_{7} \mathrm{~S}[\mathrm{M}+\mathrm{H}]^{+}$, 458.1961. Found 458.1975. 


\section{Procedure for the conversion of sulfonylimidate $7 \mathrm{~g}$ to compound 10 . To}

sulfonylimidate $7 \mathrm{~g}$ (50 $\mathrm{mg}, 0.092 \mathrm{mmol}$ ) were added a mixture of iso-propanol and $\mathrm{H}$ ${ }_{2} \mathrm{O}(95 / 5,0.5 \mathrm{ml})$ and concentrated $\mathrm{H}_{2} \mathrm{SO}_{4}(66 \mathrm{mg}$, ca. 7.0 equiv). The reaction mixture was stirred at $80{ }^{\circ} \mathrm{C}$ for $40 \mathrm{~min}$. TLC analysis revealed that no reaction had occurred, then the mixture was heated to $100{ }^{\circ} \mathrm{C}$ and stirred for $3 \mathrm{~h}$. After cooling to $\mathrm{RT}$, the mixture was diluted by addition of $\mathrm{CH}_{2} \mathrm{Cl}_{2}$. A saturated aqueous solution of $\mathrm{NaHCO}_{3}$ was added, and the mixture was extracted with $\mathrm{CH}_{2} \mathrm{Cl}_{2}$ three times. The organics were dried over anhydrous $\mathrm{MgSO}_{4}$. Filtration and removal of solvents afforded compound $\mathbf{1 0}$ (46.1 mg, quant). The obtained compound $\mathbf{1 0}$ was found to be pure by ${ }^{1} \mathrm{H}$ NMR spectroscopy analysis in DMSO-d6 (10 was very hard to dissolve in any usual organic solvents except polar solvents such as DMSO).

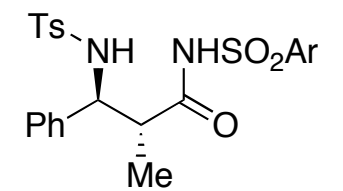

$(\operatorname{Ar}=2,5-x y l y l)$ anti-2-methyl-3-phenyl-3-( $p$-toluenesulfonylamino)- $N-(2,5-$ xylylsulfonyl)propionamide (10): $\mathrm{Mp} .218-219{ }^{\circ} \mathrm{C}$; ${ }^{1} \mathrm{H} \mathrm{NMR}$ (DMSO-d6) $\delta=12.08(\mathrm{~s}, 1 \mathrm{H}), 8.67(\mathrm{~d}, 1 \mathrm{H}, J=9.6 \mathrm{~Hz}), 7.76$ (s, $1 \mathrm{H}), 7.33-7.37(\mathrm{~m}, 1 \mathrm{H}), 7.20-7.25(\mathrm{~m}, 3 \mathrm{H}), 6.87-7.03(\mathrm{~m}, 7 \mathrm{H})$, $4.41(\mathrm{t}, 1 \mathrm{H}, J=9.9 \mathrm{~Hz}), 2.60-2.72(\mathrm{~m}, 1 \mathrm{H}), 2.50(\mathrm{~s}, 3 \mathrm{H}), 2.34(\mathrm{~s}, 3 \mathrm{H}), 2.18(\mathrm{~s}, 3 \mathrm{H})$, 0.59 (d, 3H, $J=7.4 \mathrm{~Hz}$ ); ${ }^{13} \mathrm{C}$ NMR (DMSO-d6) $\delta=172.2,141.5,138.3,137.0,135.4$, 134.0, 133.9, 132.2, 13.3, 128.6, 127.8, 126.8, 126.3, 58.9, 46.6, 20.8, 20.3, 19.1, 14.7; IR (neat) 3251, 3063, 3032, 2979, 2926, 2878, 1693, 1655, 1647, 1638, 1617 , 1599, 1560, 1542, 1493, 1457, 1382, 1341, 1288, 1223, 1201, 1163, 1123, 1087, 1062, 898, 849, 815, 766, 702, $667 \mathrm{~cm}^{-1}$; HRMS (FAB); Exact mass calcd for $\mathrm{C}_{25} \mathrm{H}_{29} \mathrm{~N}_{2} \mathrm{O}_{5} \mathrm{~S}_{2}[\mathrm{M}+\mathrm{H}]^{+}, 501.1518$. Found 501.1538.

\section{Procedure for the conversion of sulfonylimidate $7 y$ to ester 11. To}

sulfonylimidate $7 \mathbf{y}(82.3 \mathrm{mg}, 0190 \mathrm{mmol})$ were added a mixture of DMF and $\mathrm{H}_{2} \mathrm{O}$ $(95 / 5,0.38 \mathrm{ml})$, and a solution of DBU in DMF (10 mol\%, $30 \mu \mathrm{l})$. The mixture was stirred at RT for $33 \mathrm{~h}$, and then diluted by addition of $\mathrm{Et}_{2} \mathrm{O}$. The mixture was washed with water 3 times, then dried over anhydrous $\mathrm{Na}_{2} \mathrm{SO}_{4}$. Filtration and removal of solvents afforded the crude product. Purification of the crude product was conducted by chromatography on $\mathrm{SiO}_{2}$, to afford the desired ester $\mathbf{1 1}$ (50.4 mg, 90\% yield).

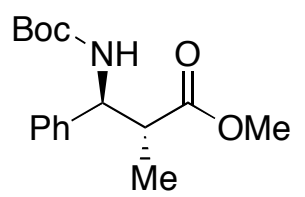

tert-Butyl anti-2-(methoxycarbonyl)-1-phenylpropylcarbamate (11): Mp. 89-90 ${ }^{\circ} \mathrm{C} ;{ }^{1} \mathrm{H}$ NMR $\left(\mathrm{CDCl}_{3}\right) \delta=7.28-7.34(\mathrm{~m}, 2 \mathrm{H})$, 7.20-7.27 (m, 3H), $5.78(\mathrm{~s}, 1 \mathrm{H}), 4.83(\mathrm{~s}, 1 \mathrm{H}), 3.58(\mathrm{~s}, 3 \mathrm{H}), 2.92(\mathrm{~s}$, $1 \mathrm{H}), 1.42(\mathrm{~s}, 9 \mathrm{H}), 1.23(\mathrm{~d}, 3 \mathrm{H}, J=6.2 \mathrm{~Hz}) ;{ }^{13} \mathrm{C} \mathrm{NMR}\left(\mathrm{CDCl}_{3}\right) \delta=175.3,155.4$, 127.3, 126.6, 126.2 , 79.4, 51.6, 45.2, 28.3, 15.3; IR (neat) 3428. 3360, 2977, 2356, $1716,1497,1455,1434,1365,1289,1245,1168,1085,1051,1005,879,756,701$, 
$585 \mathrm{~cm}^{-1}$; HRMS (FAB); Exact mass calcd for $\mathrm{C}_{16} \mathrm{H}_{24} \mathrm{NO}_{4}[\mathrm{M}+\mathrm{H}]^{+}, 294.1705$. Found 294.1696.

\section{Procedure for Red-Al reduction of sulfonylimidates $7 \mathrm{~d}$ and $7 \mathrm{~g}$ to aldehydes $12 \mathrm{a}$}

and 12b. The procedure for the conversion of $\mathbf{7 d}$ to $\mathbf{1 2 a}$ is as follows. A solution of 7d (100 mg, $0.205 \mathrm{mmol})$ in $2 \mathrm{ml}$ of THF was cooled to $-70^{\circ} \mathrm{C}$, and then Red-Al ( $65 \% \mathrm{w} / \mathrm{w}$ toluene solution, $385 \mu \mathrm{l}, 7.0$ equiv) was added slowly lest the temperature should increase. The mixture was stirred at $-70{ }^{\circ} \mathrm{C}$ for $18 \mathrm{~h}$, and the reaction was quenched by addition of $\mathrm{MeOH}(0.1 \mathrm{ml})$ at $-70^{\circ} \mathrm{C}$. The mixture was stirred for $5 \mathrm{~min}$ at that temperature, and $\mathrm{H}_{2} \mathrm{O}$ was added. The temperature was allowed to increase to $\mathrm{RT}$, and AcOEt and a saturated aqueous solution of $\mathrm{NH}_{4} \mathrm{Cl}$ were added. After filtration, the obtained filtrate was extracted with AcOEt three times, and the organics were dried over anhydrous $\mathrm{Na}_{2} \mathrm{SO}_{4}$. Filtration and removal of solvents afforded the crude product. Purification of the crude product was conducted by chromatography on neutral $\mathrm{SiO}_{2}$, to afford the aldehyde 12a (46.9 mg, $87 \%$ yield). Aldehyde 12b was obtained by following the same procedure mentioned above ( $83 \%$ yield).

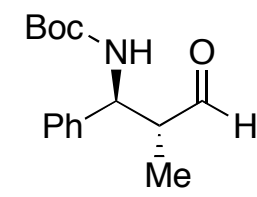

tert-butyl anti-2-formyl-1-phenylpropylcarbamate (12a): Mp. 82-83 ${ }^{\circ} \mathrm{C} ;{ }^{1} \mathrm{H}$ NMR $\left(\mathrm{CDCl}_{3}\right) \delta=9.66(\mathrm{~d}, 1 \mathrm{H} . J=2.3 \mathrm{~Hz}), 7.22-7.37$ $(\mathrm{m}, 5 \mathrm{H}), 5.28(\mathrm{~s}, 1 \mathrm{H}), 4.78(\mathrm{~s}, 1 \mathrm{H}), 2.81(\mathrm{~s}, 1 \mathrm{H}), 1.39(\mathrm{~s}, 9 \mathrm{H}), 1.02(\mathrm{~d}$, $3 \mathrm{H}, J=6.8 \mathrm{~Hz}) ;{ }^{13} \mathrm{C} \mathrm{NMR}\left(\mathrm{CDCl}_{3}\right) \delta=203.3,155.2,139.9,128.7,127.7,126.8,79.9$, 52.1, 28.2, 11.8; IR (neat) 3343, 2978, 2933, 2817, 2723, 1716, 1699, 1519, 1507, 1455, 1392, 1366, 1288, 1249, 1169, 1086, 1002, 755, $701 \mathrm{~cm}^{-1}$; HRMS (FAB); Exact mass calcd for $\mathrm{C}_{15} \mathrm{H}_{22} \mathrm{NO}_{3}[\mathrm{M}+\mathrm{H}]^{+}$, 264.1599. Found 264.1606.

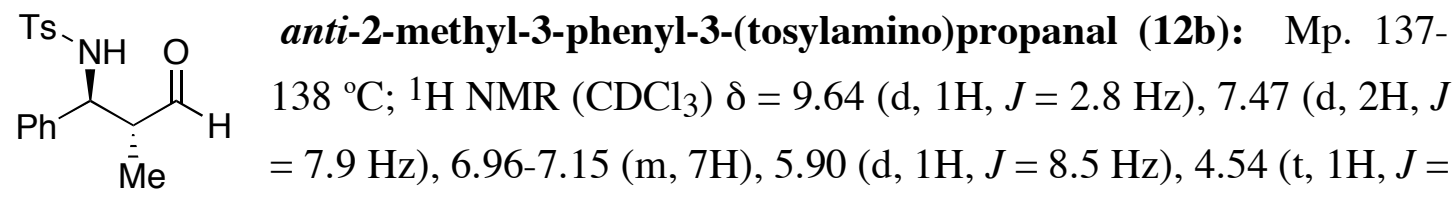
$8.5 \mathrm{~Hz}), 2.75-2.84(\mathrm{~m}, 1 \mathrm{H}), 2.30(\mathrm{~s}, 3 \mathrm{H}), 0.96(\mathrm{~d}, 3 \mathrm{H}, J=6.8 \mathrm{~Hz}) ;{ }^{13} \mathrm{C} \mathrm{NMR}\left(\mathrm{CDCl}_{3}\right)$ $\delta=203.2,143.0,137.8,137.2,129.2,128.4,127.6,127.0,127.0,59.1,51.9,21.3$, 11.8; IR (neat) 3268, 3068, 3030, 2972, 2926, 2872, 2727, 1724, 1598, 1495, 1456, 1325, 1185, 1160, 1090, 1045, 914, 812, 761, 702, 670, 566, $547 \mathrm{~cm}^{-1}$; HRMS (FAB); Exact mass calcd for $\mathrm{C}_{17} \mathrm{H}_{20} \mathrm{NSO}_{3}[\mathrm{M}+\mathrm{H}]^{+}, 318.1184$. Found 318.1169. 


\section{Procedure for direct formation of ester $\mathbf{1 3}$ from benzaldehyde and}

sulfonylimidate $6 \mathbf{h}$. To a solution of benzaldehyde $(60.9 \mu \mathrm{l}, 0.6 \mathrm{mmol})$ and DBU (18.3 mg, $0.12 \mathrm{mmol}$ ) in $0.2 \mathrm{ml}$ of DMF was added 2,5-xylylsulfonylamide (11.1 mg, $0.06 \mathrm{mmol}$ ). To the mixture was slowly added over $32 \mathrm{~h}$ a solution of sulfonylimidate 6h $(183.8 \mathrm{mg}, 0.72 \mathrm{mmol})$ in $1 \mathrm{ml}$ of DMF. After completion of slow addition, the mixture was stirred at RT for further $46 \mathrm{~h}$ (total $78 \mathrm{~h}$ ), and then diluted by addition of $\mathrm{Et}_{2} \mathrm{O}$. The mixture was washed with water 3 times, then dried over anhydrous $\mathrm{Na}_{2} \mathrm{SO}_{4}$. Filtration and removal of solvents afforded the crude product. Purification of the crude product was conducted by chromatography on $\mathrm{SiO}_{2}$, to afford the desired ester 13 (diastereomers mixture, $196.2 \mathrm{mg}, 90 \%$ yield, anti/syn = 87/13).

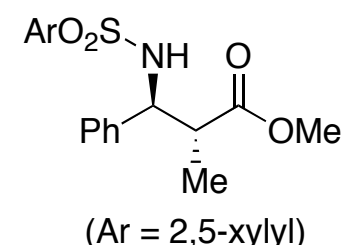

\section{Methyl}

anti-2-methyl-3-phenyl-3-(2,5xylylsulfonylamino)propionate (13): Mp. $116-117{ }^{\circ} \mathrm{C} ;{ }^{1} \mathrm{H}$ $\operatorname{NMR}\left(\mathrm{CDCl}_{3}\right) \delta=7.49(\mathrm{~s}, 1 \mathrm{H}), 7.09-7.13(\mathrm{~m}, 3 \mathrm{H}), 7.06(\mathrm{~d}, 1 \mathrm{H}$,

$$
(\mathrm{Ar}=2,5-\mathrm{xyly}) \quad J=7.4 \mathrm{~Hz}), 6.93-7.02(\mathrm{~m}, 3 \mathrm{H}), 6.03(\mathrm{~d}, 1 \mathrm{H}, J=8.5 \mathrm{~Hz}), 4.44(\mathrm{t} \text {, }
$$

$1 \mathrm{H}, J=7.7 \mathrm{~Hz}), 3.60(\mathrm{~s}, 1 \mathrm{H}), 2.80-2.90(\mathrm{~m}, 1 \mathrm{H}), 2.47(\mathrm{~s}, 3 \mathrm{H}), 2.20(\mathrm{~s}, 3 \mathrm{H}), 1.09$ (d, $3 \mathrm{H}, J=6.8 \mathrm{~Hz}) ;{ }^{13} \mathrm{C} \mathrm{NMR}\left(\mathrm{CDCl}_{3}\right) \delta=175.0,138.7,138.3,135.6,133.4,132.8$, 132.0, 129.5, 128.2, 127.6, 126.5, 60.4, 51.9, 45.8, 20.5, 19.7, 15.3; IR (neat) 3292, 2983, 2953, 2878, 1734, 1717, 1540, 1507, 1489, 1456, 1436, 1322, 1278, 1206, 1158, 1063, 916, 818, 766, 751, 701, 616, $594 \mathrm{~cm}^{-1}$; HRMS (FAB); Exact mass calcd for $\mathrm{C}_{19} \mathrm{H}_{24} \mathrm{NSO}_{4}[\mathrm{M}+\mathrm{H}]^{+}, 362.1426$. Found 362.1419.

\section{Origin of high anti-selectivity}

Single crystal X-ray diffraction analysis of a sulfonylimidate (6a) and some of the Mannich adducts (syn-7a, anti-7d, anti-7g, and syn-7g), not only provided information about the relative configuration of the products, but also characteristic structure of the sulfonylimidate group. In every case the imine adopts an $E$ configuration and the oxygen at the carbon of the $\mathrm{C}=\mathrm{N}$ bond is $\mathrm{sp}^{2}$ hybridised, and displays an s-cis geometry about the N-C-O-C bonds. ${ }^{6}$ This phenomena can be rationalized by considering $n-\sigma^{*}$ interaction between oxygen lone electron pair and $\sigma^{*}$ anti-bonding orbital of $\mathrm{C}=\mathrm{N}$ group. Predominant $E$-configuration of the imine may stem from the steric repulsion of the alkyl group on oxygen and the sulfonyl group.

The proposed mechanism for anti-selectivity is demonstrated in Figure 1. Based on the experimental information that the imine adopts an $E$ configuration, the geometry of the generated aza-enolate is expected to be $Z$ (Scheme 4). Considering the steric repulsion between the methyl substituent and the Boc group (repulsion $c$ ), and that between the $\mathrm{SO}_{2} \mathrm{Ar}$ group and the $\mathrm{R}$ group (repulsion $b$ ) in TS-2 led us to the conclusion that TS-1 may be favored to give anti product. When pivalaldehyde-

\footnotetext{
${ }^{6}$ Neilson, D. G. In The Chemistry of Amidines and Imidates; Patai, S., Ed.; John Wiley \& Sons: London, UK, 1975; Chapter 8.
} 
derived Ts imine was used (Table 2, entry 14), syn-selectivity was observed, which may be rationalized by considering that the repulsion between $t \mathrm{Bu}$ group and $\mathrm{Me}$ substituent (repulsion $a$ in TS-1) is large in Ts-1. In $\mathbf{1 4}$ the formation of s-trans configuration about the S-N-C-C bonds would be suppressed by steric repulsion between the $i \operatorname{Pr}$ group and the methyl substituent (repulsion $d$ ), while the s-trans formation may be possible in the case of a sulfonylimidate bearing less sterically demanding methoxy group, which may be the reason for the observed low antiselectivity (Table 1, entry 4).
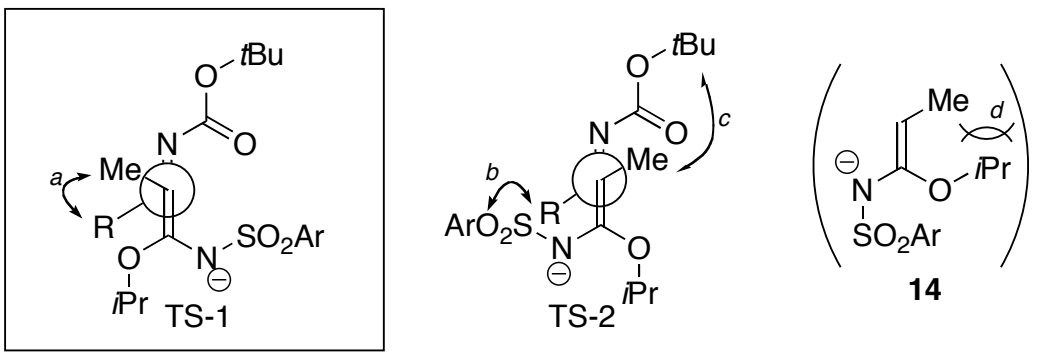

Figure 1. Proposed Transition State Model.

Scheme 4. Proposed Geometry of Aza-enolate Derived from Sulfonylimidate.

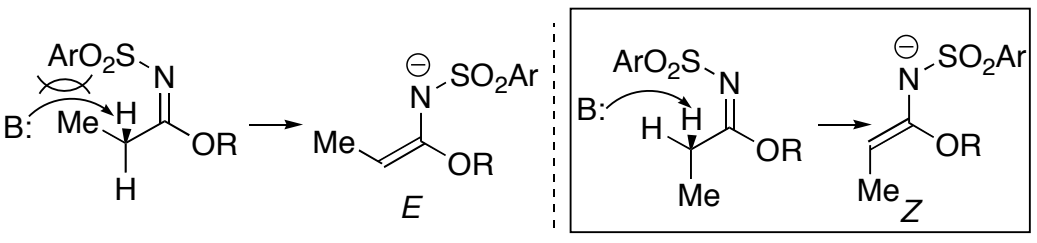

\section{Proposed Catalytic Cycle for the Direct Formation of $\beta$-Amino Acid Derivative. $\mathrm{PhCHO}$}

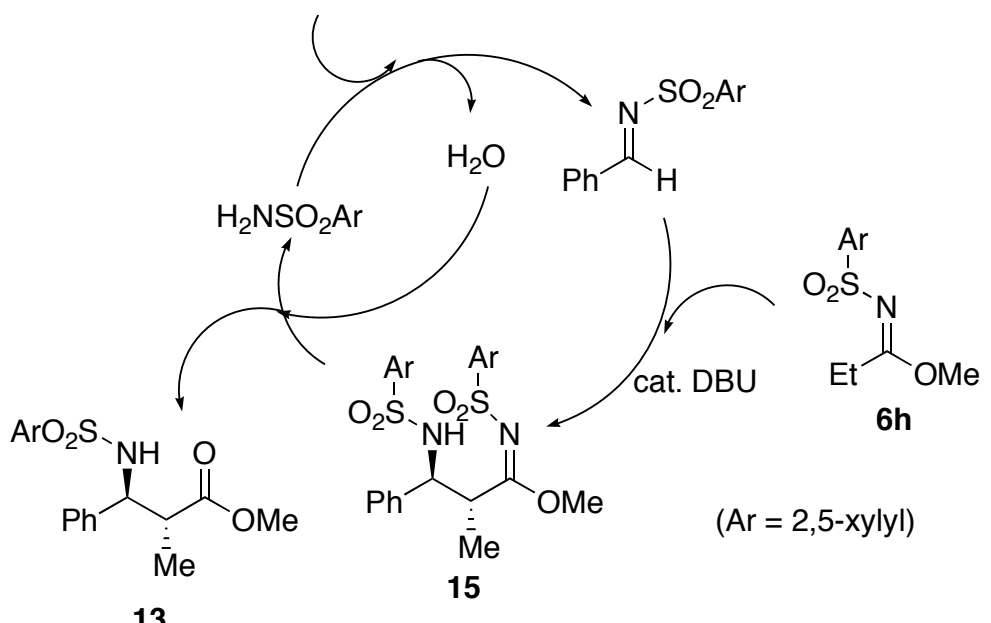

The fact that intermediate $\mathbf{1 5}$ could be isolated if slow addition of $\mathbf{6 h}$ was not conducted, supports this proposed reaction mechanism. The structure of product $\mathbf{1 3}$ was unambiguously determined by X-ray diffraction analysis. 


\section{X-ray Diffraction Analysis}

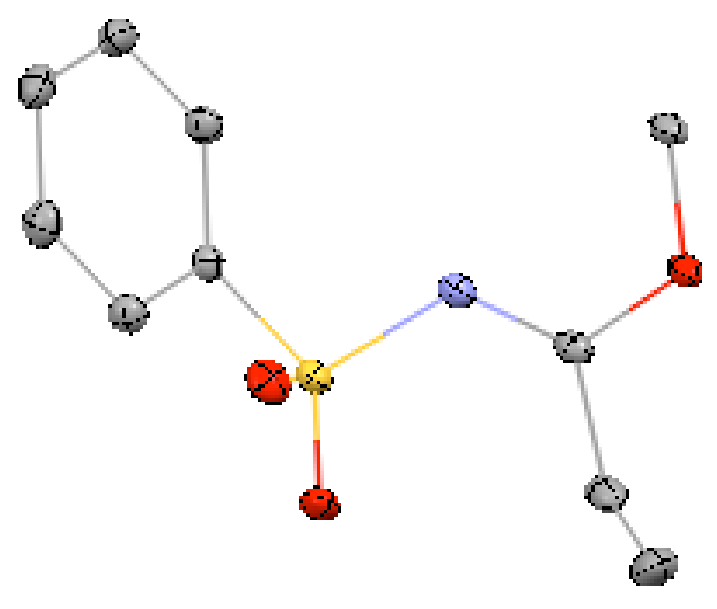

$6 \mathbf{a}$

A. Crystal Data

Empirical Formula

Formula Weight

Crystal Color, Habit

Crystal Dimensions

Crystal System

Lattice Type

Indexing Images

Detector Position

Pixel Size

Lattice Parameters
$\mathrm{C}_{10} \mathrm{H}_{13} \mathrm{NSO}_{3}$

227.28

colorless, block

$0.40 \times 0.30 \times 0.20 \mathrm{~mm}$

monoclinic

Primitive

3 oscillations @ 90.0 seconds

$127.40 \mathrm{~mm}$

$0.100 \mathrm{~mm}$

$$
\begin{aligned}
& a=11.0472(4) \AA \\
& b=8.8900(3) \AA \\
& c=12.0126(4) \AA \\
& \beta=109.5182(10) 0 \\
& V=1111.96(6) \AA^{3}
\end{aligned}
$$


Space Group

$Z$ value

Dcalc

F000

$\mu(\operatorname{MoK} \alpha)$
$\mathrm{P} 21 / \mathrm{n}(\# 14)$

4

$1.358 \mathrm{~g} / \mathrm{cm}^{3}$

480.00

$2.776 \mathrm{~cm}^{-1}$ 
B. Intensity Measurements

Diffractometer

Radiation

Detector Aperture

Data Images

$\omega$ oscillation Range $(\chi=45.0, \phi=0.0)$

Exposure Rate

$\omega$ oscillation Range $(\chi=45.0, \phi=180.0)$

Exposure Rate

Detector Position

Pixel Size

$2 \theta \max$

No. of Reflections Measured

Corrections
Rigaku RAXIS-RAPID

$\operatorname{MoK} \alpha(\lambda=0.71075 \AA)$

graphite monochromated

$280 \mathrm{~mm} \times 256 \mathrm{~mm}$

44 exposures

$130.0-190.0^{\circ}$

$30.0 \mathrm{sec} . / 0$

$0.0-160.0^{\circ}$

$30.0 \mathrm{sec} . / \mathrm{O}$

$127.40 \mathrm{~mm}$

$0.100 \mathrm{~mm}$

54.90

Total: 10581

Unique: $2544(\operatorname{Rint}=0.029)$

Lorentz-polarization

Absorption

(trans. factors: $0.807-0.946$ ) 
C. Structure Solution and Refinement

Structure Solution

Refinement

Function Minimized

Least Squares Weights

$.5000] /\left(4 \mathrm{Fo}^{2}\right)$

$2 \theta$ max cutoff

Anomalous Dispersion

No. Observations (All reflections)

No. Variables

Reflection/Parameter Ratio

Residuals: R1 (I>2.00\%(I))

Residuals: $\mathrm{R}$ (All reflections)

Residuals: wR2 (All reflections)

Goodness of Fit Indicator

Max Shift/Error in Final Cycle

Maximum peak in Final Diff. Map

Minimum peak in Final Diff. Map
Direct Methods

Full-matrix least-squares on $\mathrm{F}^{2}$

$\Sigma w\left(\mathrm{Fo}^{2}-\mathrm{Fc}^{2}\right)^{2}$

$1 /\left[0.0010 \mathrm{Fo}^{2}+3.0000 \mathrm{~s}\left(\mathrm{Fo}^{2}\right)+0\right.$

54.90

All non-hydrogen atoms

2544

188

13.53

0.0294

0.0314

0.0874

0.805

0.000

$0.30 \mathrm{e} / \AA^{3}$

-0.47 e/Å 3 


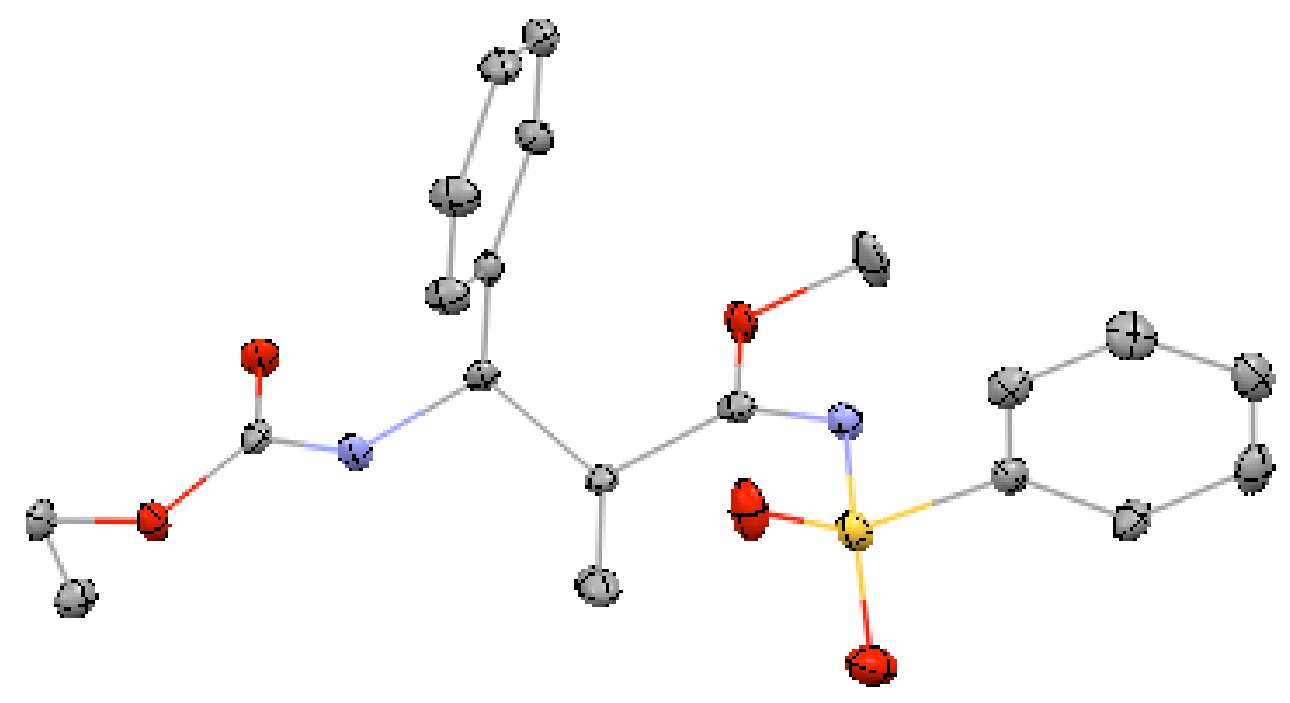

syn-7a

A. Crystal Data

Empirical Formula

Formula Weight

Crystal Color, Habit

Crystal Dimensions

Crystal System

Lattice Type

Indexing Images

Detector Position

Pixel Size

Lattice Parameters
$\mathrm{C}_{20} \mathrm{H}_{24} \mathrm{~N}_{2} \mathrm{O}_{5} \mathrm{~S}$

404.48

colorless, block

$0.30 \times 0.30 \times 0.30 \mathrm{~mm}$

triclinic

Primitive

3 oscillations @ 90.0 seconds

$127.40 \mathrm{~mm}$

$0.100 \mathrm{~mm}$

$$
\begin{aligned}
& a=9.6790(10) \AA \\
& b=9.7118(9) \AA \\
& c=22.524(3) \AA \\
& \alpha=87.724(3) \circ \\
& \beta=77.352(3) \circ \\
& \gamma=79.122(3) \circ \\
& V=2028.8(4) \AA^{3}
\end{aligned}
$$


Space Group

$Z$ value

D calc

F000

$\mu(\operatorname{MoK} \alpha)$
P-1 (\#2)

4

$1.324 \mathrm{~g} / \mathrm{cm}^{3}$

856.00

$1.928 \mathrm{~cm}^{-1}$ 
B. Intensity Measurements

Diffractometer

Radiation

Detector Aperture

Data Images

$\omega$ oscillation Range $(\chi=45.0, \phi=30.0)$

Exposure Rate

$\omega$ oscillation Range $(\chi=45.0, \phi=210.0)$

Exposure Rate

Detector Position

Pixel Size

$2 \theta \max$

No. of Reflections Measured

Corrections
Rigaku RAXIS-RAPID

$\operatorname{MoK} \alpha(\lambda=0.71075 \AA)$

graphite monochromated

$280 \mathrm{~mm} \times 256 \mathrm{~mm}$

44 exposures

$130.0-190.0^{\circ}$

$60.0 \mathrm{sec} . / 0$

$0.0-160.0^{\circ}$

$60.0 \mathrm{sec} . / \mathrm{O}$

$127.40 \mathrm{~mm}$

$0.100 \mathrm{~mm}$

$55.0^{\circ}$

Total: 19196

Unique: $9141(\operatorname{Rint}=0.111)$

Lorentz-polarization

Absorption

(trans. factors: $0.936-0.944$ ) 
C. Structure Solution and Refinement

Structure Solution

Refinement

Function Minimized

Least Squares Weights

$2 \theta$ max cutoff

Anomalous Dispersion

No. Observations (All reflections)

No. Variables

Reflection/Parameter Ratio

Residuals: R1 (I>2.00\%(I))

Residuals: R (All reflections)

Residuals: wR2 (All reflections)

Goodness of Fit Indicator

Max Shift/Error in Final Cycle

Maximum peak in Final Diff. Map

Minimum peak in Final Diff. Map
Direct Methods

Full-matrix least-squares on $\mathrm{F}^{2}$

$\Sigma w\left(\mathrm{Fo}^{2}-\mathrm{Fc}^{2}\right)^{2}$

$1 /\left[0.0078 \mathrm{Fo}^{2}+1.0000 \mathrm{~s}\left(\mathrm{Fo}^{2}\right)\right] /\left(4 \mathrm{Fo}^{2}\right)$

$55.0^{0}$

All non-hydrogen atoms

9141

553

16.53

0.1204

0.1445

0.3145

0.987

0.004

$1.98 \mathrm{e} / \mathrm{A}^{3}$

$-1.44 \mathrm{e} / \AA^{3}$ 


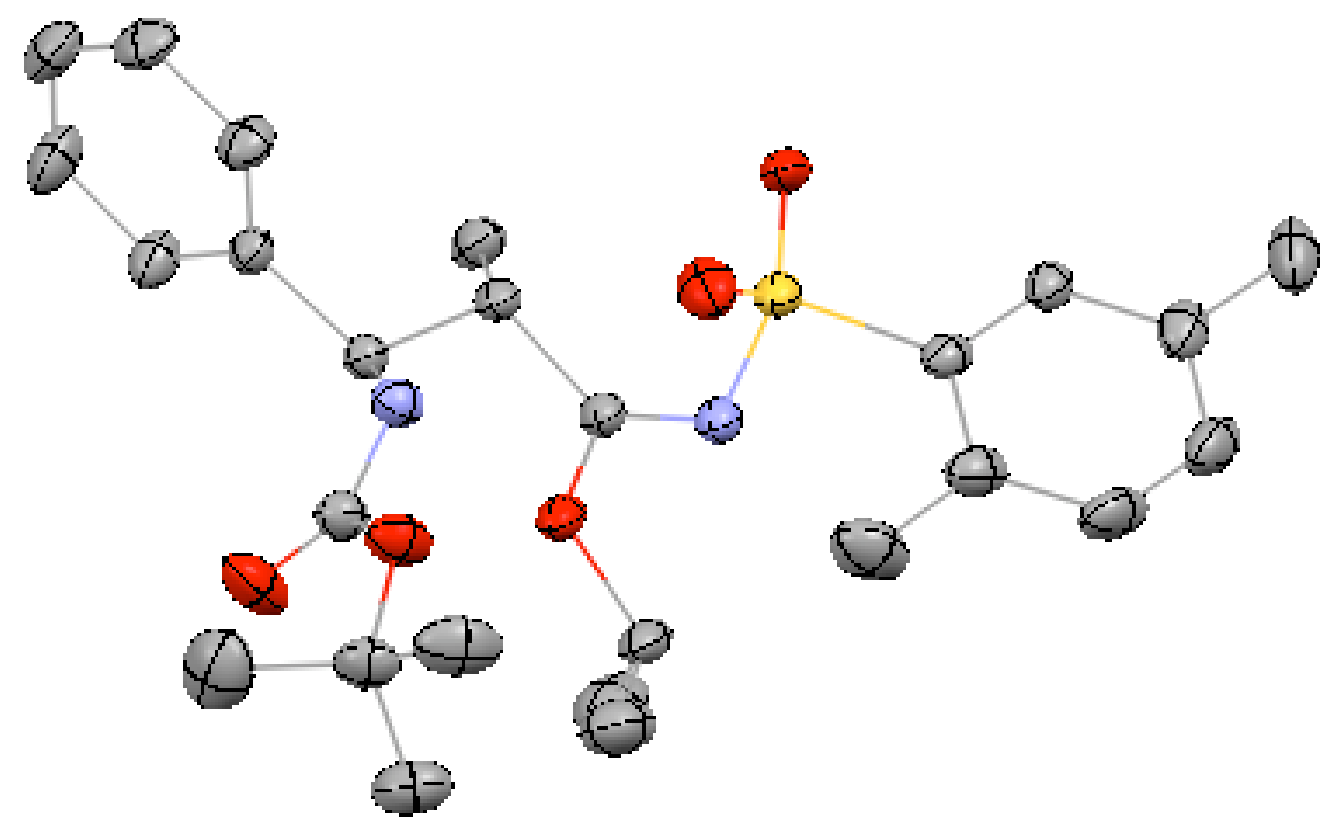

anti-7d

A. Crystal Data

Empirical Formula

Formula Weight

Crystal Color, Habit

Crystal Dimensions

Crystal System

Lattice Type

Indexing Images

Detector Position

Pixel Size

Lattice Parameters
$\mathrm{C}_{26} \mathrm{H}_{36} \mathrm{~N}_{2} \mathrm{O}_{5} \mathrm{~S}$

488.64

colorless, block

$0.20 \times 0.10 \times 0.10 \mathrm{~mm}$

triclinic

Primitive

3 oscillations @ 90.0 seconds

$127.40 \mathrm{~mm}$

$0.100 \mathrm{~mm}$

$$
\begin{aligned}
& a=8.6250(6) \AA \\
& b=10.2643(7) \AA \\
& c=17.2442(13) \AA \\
& \alpha=85.8573(19) \circ \\
& \beta=85.7269(18) \circ \\
& \gamma=65.8507(16) \circ
\end{aligned}
$$




$$
V=1387.69(17) \AA^{3}
$$

Space Group

$Z$ value

$\mathrm{D}_{\text {calc }}$

$F_{000}$

$\mu(\operatorname{MoK} \alpha)$
P-1 (\#2)

2

$1.169 \mathrm{~g} / \mathrm{cm}^{3}$

524.00

$1.519 \mathrm{~cm}^{-1}$ 


\section{B. Intensity Measurements}

Diffractometer

Radiation

Detector Aperture

Data Images

$\omega$ oscillation Range $(\chi=45.0, \phi=30.0)$

Exposure Rate

$\omega$ oscillation Range $(\chi=45.0, \phi=180.0)$

Exposure Rate

Detector Position

Pixel Size

$2 \theta_{\max }$

No. of Reflections Measured

Corrections
Rigaku RAXIS-RAPID

$\operatorname{MoK} \alpha(\lambda=0.71075 \AA)$

graphite monochromated

$280 \mathrm{~mm} \times 256 \mathrm{~mm}$

44 exposures

$130.0-190.0^{\circ}$

$60.0 \mathrm{sec} . / 0$

$0.0-160.0^{0}$

$60.0 \mathrm{sec} . / 0$

$127.40 \mathrm{~mm}$

$0.100 \mathrm{~mm}$

54.90

Total: 13534

Unique: $6242\left(\mathrm{R}_{\mathrm{int}}=0.028\right)$

Lorentz-polarization

Absorption

(trans. factors: 0.913 - 0.985) 
C. Structure Solution and Refinement

Structure Solution

Refinement

Function Minimized

Least Squares Weights

$2 \theta_{\max }$ cutoff

Anomalous Dispersion

No. Observations (All reflections)

No. Variables

Reflection/Parameter Ratio

Residuals: R1 (I>2.00\%(I))

Residuals: R (All reflections)

Residuals: wR2 (All reflections)

Goodness of Fit Indicator

Max Shift/Error in Final Cycle

Maximum peak in Final Diff. Map

Minimum peak in Final Diff. Map
Patterson Methods (SHELXS86)

Full-matrix least-squares on $\mathrm{F}^{2}$

$\Sigma w\left(F^{2}-F_{c}^{2}\right)^{2}$

$w=1 /\left[\sigma^{2}\left(\mathrm{Fo}^{2}\right)+(0.1229 \cdot P)^{2}\right.$

$+0.0000 \cdot P]$

where $\mathrm{P}=\left(\operatorname{Max}\left(\mathrm{Fo}^{2}, 0\right)+2 \mathrm{Fc}^{2}\right) / 3$

$54.9^{\circ}$

All non-hydrogen atoms

6242

312

20.01

0.0600

0.1065

0.2098

1.047

0.000

$0.39 \mathrm{e} / \AA^{3}$

-0.37 e/Å3 


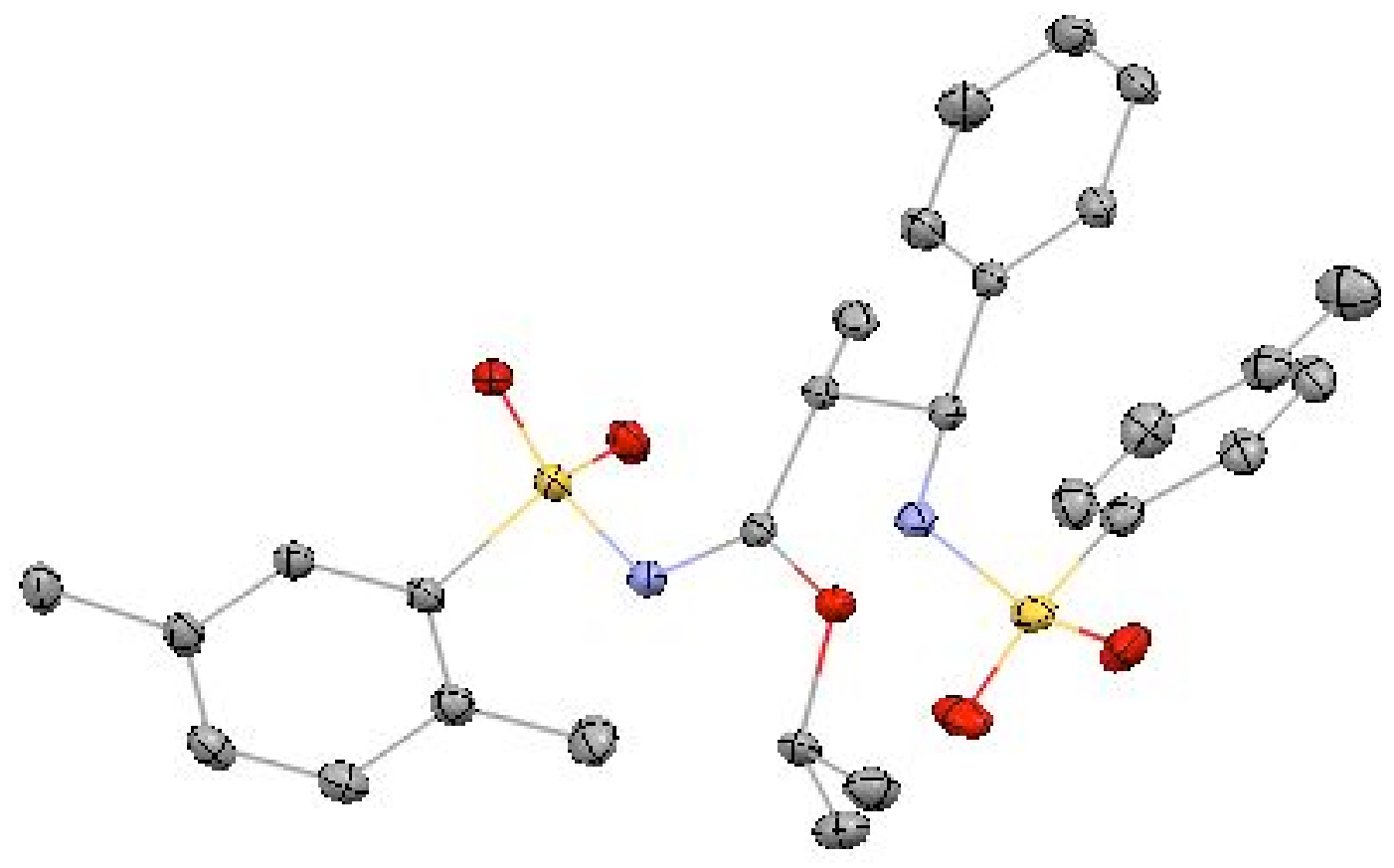

anti-7g

A. Crystal Data

Empirical Formula

Formula Weight

Crystal Color, Habit

Crystal Dimensions

Crystal System

Lattice Type

Indexing Images

Detector Position

Pixel Size

Lattice Parameters
$\mathrm{C}_{28} \mathrm{H}_{34} \mathrm{~N}_{2} \mathrm{~S}_{2} \mathrm{O}_{5}$

542.71

colorless, block

$0.20 \times 0.20 \times 0.20 \mathrm{~mm}$

monoclinic

Primitive

3 oscillations @ 90.0 seconds

$127.40 \mathrm{~mm}$

$0.100 \mathrm{~mm}$

$$
\begin{aligned}
& a=9.2941(8) \AA \\
& b=19.0359(17) \AA \\
& c=16.2694(14) \AA \\
& \beta=102.4056(18) 0 \\
& V=2811.2(4) \AA^{3}
\end{aligned}
$$


Space Group

$Z$ value

$D_{\text {calc }}$

$F_{000}$

$\mu(\mathrm{MoK} \alpha)$
$\mathrm{P} 21 / \mathrm{n}$ (\#14)

4

$1.282 \mathrm{~g} / \mathrm{cm}^{3}$

1152.00

$2.288 \mathrm{~cm}^{-1}$ 


\section{B. Intensity Measurements}

Diffractometer

Radiation

Detector Aperture

Data Images

$\omega$ oscillation Range $(\chi=45.0, \phi=0.0)$

Exposure Rate

$\omega$ oscillation Range $(\chi=45.0, \phi=180.0)$

Exposure Rate

Detector Position

Pixel Size

$2 \theta_{\max }$

No. of Reflections Measured

Corrections
Rigaku RAXIS-RAPID

$\operatorname{MoK} \alpha(\lambda=0.71075 \AA)$

graphite monochromated

$280 \mathrm{~mm} \times 256 \mathrm{~mm}$

44 exposures

$130.0-190.0^{0}$

$30.0 \mathrm{sec} . / 0$

$0.0-160.0^{0}$

$30.0 \mathrm{sec} . / 0$

$127.40 \mathrm{~mm}$

$0.100 \mathrm{~mm}$

54.90

Total: 26494

Unique: $6381\left(R_{\text {int }}=0.051\right)$

Lorentz-polarization

Absorption

(trans. factors: 0.905 - 0.955) 

C. Structure Solution and Refinement

Structure Solution

Refinement

Function Minimized

Least Squares Weights

$2 \theta_{\max }$ cutoff

Anomalous Dispersion

No. Observations (All reflections)

No. Variables

Reflection/Parameter Ratio

Residuals: R1 (I>2.00\%(I))

Residuals: $\mathrm{R}$ (All reflections)

Residuals: wR2 (All reflections)

Goodness of Fit Indicator

Max Shift/Error in Final Cycle

Maximum peak in Final Diff. Map

Minimum peak in Final Diff. Map
Direct Methods (SHELX97)

Full-matrix least-squares on $\mathrm{F}^{2}$

$\Sigma w\left(F^{2}-F_{c}^{2}\right)^{2}$

$w=1 /\left[\sigma^{2}\left(F^{2}\right)+(0.0625 \cdot P)^{2}\right.$

$+0.6498 \cdot P]$

where $\mathrm{P}=\left(\operatorname{Max}\left(\mathrm{Fo}^{2}, 0\right)+2 \mathrm{Fc}^{2}\right) / 3$

54.90

All non-hydrogen atoms

6381

341

18.71

0.0429

0.0514

0.1207

1.084

0.001

$0.30 \mathrm{e} / \AA^{3}$

$-0.54 \mathrm{e} / \mathrm{A}^{3}$ 


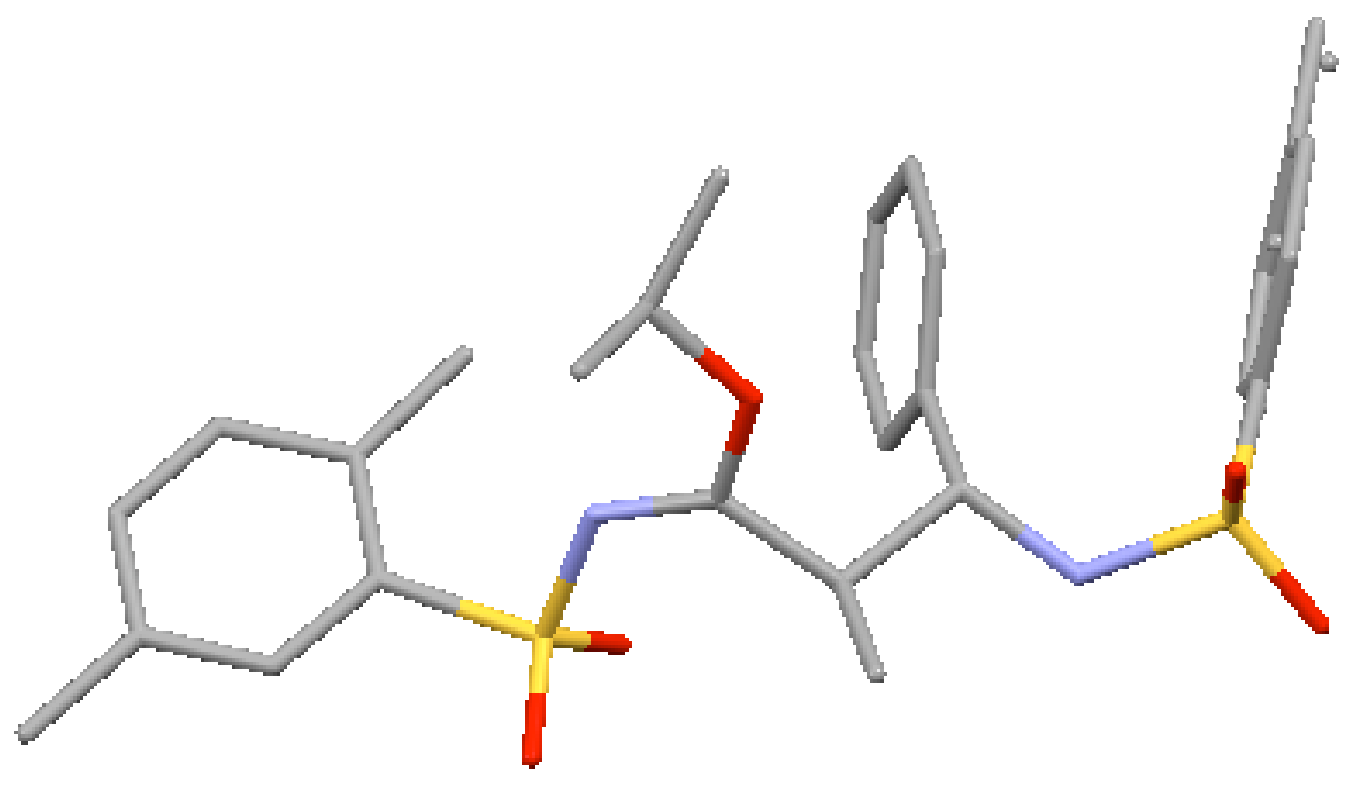

syn-7g

A. Crystal Data

Empirical Formula

Formula Weight

Crystal Color, Habit

Crystal Dimensions

Crystal System

Lattice Type

Indexing Images

Detector Position

Pixel Size

Lattice Parameters
$\mathrm{C}_{28} \mathrm{H}_{29} \mathrm{~N}_{2} \mathrm{~S}_{2} \mathrm{O}_{5}$

537.67

colorless, block

$0.20 \times 0.20 \times 0.20 \mathrm{~mm}$

triclinic

Primitive

3 oscillations @ 90.0 seconds

$127.40 \mathrm{~mm}$

$0.100 \mathrm{~mm}$

$$
\begin{aligned}
& a=9.4702(5) \AA \\
& b=12.0832(7) \AA \\
& c=14.1583(8) \AA \\
& \alpha=105.7265(16) \circ \\
& \beta=106.9474(15) \circ \\
& \gamma=99.1637(17) \circ \\
& V=1440.95(14) \AA^{3}
\end{aligned}
$$


Space Group

$Z$ value

$\mathrm{D}_{\text {calc }}$

$F_{000}$

$\mu(\mathrm{MoK} \alpha)$
P-1 (\#2)

2

$1.239 \mathrm{~g} / \mathrm{cm}^{3}$

566.00

$2.227 \mathrm{~cm}^{-1}$ 


\section{B. Intensity Measurements}

Diffractometer

Radiation

Detector Aperture

Data Images

$\omega$ oscillation Range $(\chi=45.0, \phi=0.0)$

Exposure Rate

$\omega$ oscillation Range $(\chi=45.0, \phi=180.0)$

Exposure Rate

Detector Position

Pixel Size

$2 \theta_{\max }$

No. of Reflections Measured

Corrections
Rigaku RAXIS-RAPID

$\operatorname{MoK} \alpha(\lambda=0.71075 \AA)$

graphite monochromated

$280 \mathrm{~mm} \times 256 \mathrm{~mm}$

44 exposures

$130.0-190.0^{0}$

$120.0 \mathrm{sec} . / \mathrm{O}$

$0.0-160.0^{0}$

$120.0 \mathrm{sec} . / 0$

$127.40 \mathrm{~mm}$

$0.100 \mathrm{~mm}$

54.90

Total: 14126

Unique: $6518\left(\mathrm{R}_{\mathrm{int}}=0.020\right)$

Lorentz-polarization

Absorption

(trans. factors: 0.739 - 0.956) 

C. Structure Solution and Refinement

Structure Solution

Refinement

Function Minimized

Least Squares Weights

$2 \theta_{\max }$ cutoff

Anomalous Dispersion

No. Observations (All reflections)

No. Variables

Reflection/Parameter Ratio

Residuals: R1 (I>2.00\%(I))

Residuals: $\mathrm{R}$ (All reflections)

Residuals: wR2 (All reflections)

Goodness of Fit Indicator

Max Shift/Error in Final Cycle

Maximum peak in Final Diff. Map

Minimum peak in Final Diff. Map
Direct Methods (SHELX97)

Full-matrix least-squares on $\mathrm{F}^{2}$

$\Sigma w\left(F^{2}-F_{c}^{2}\right)^{2}$

$w=1 /\left[\sigma^{2}\left(F^{2}\right)+(0.0818 \cdot P)^{2}\right.$

$+0.3155 \cdot P]$

where $\mathrm{P}=\left(\operatorname{Max}\left(\mathrm{Fo}^{2}, 0\right)+2 \mathrm{Fc}^{2}\right) / 3$

$54.9^{\circ}$

All non-hydrogen atoms

6518

302

21.58

0.0508

0.0610

0.1552

1.100

0.001

$0.41 \mathrm{e} / \AA^{3}$

$-0.41 \mathrm{e} / \mathrm{A}^{3}$ 


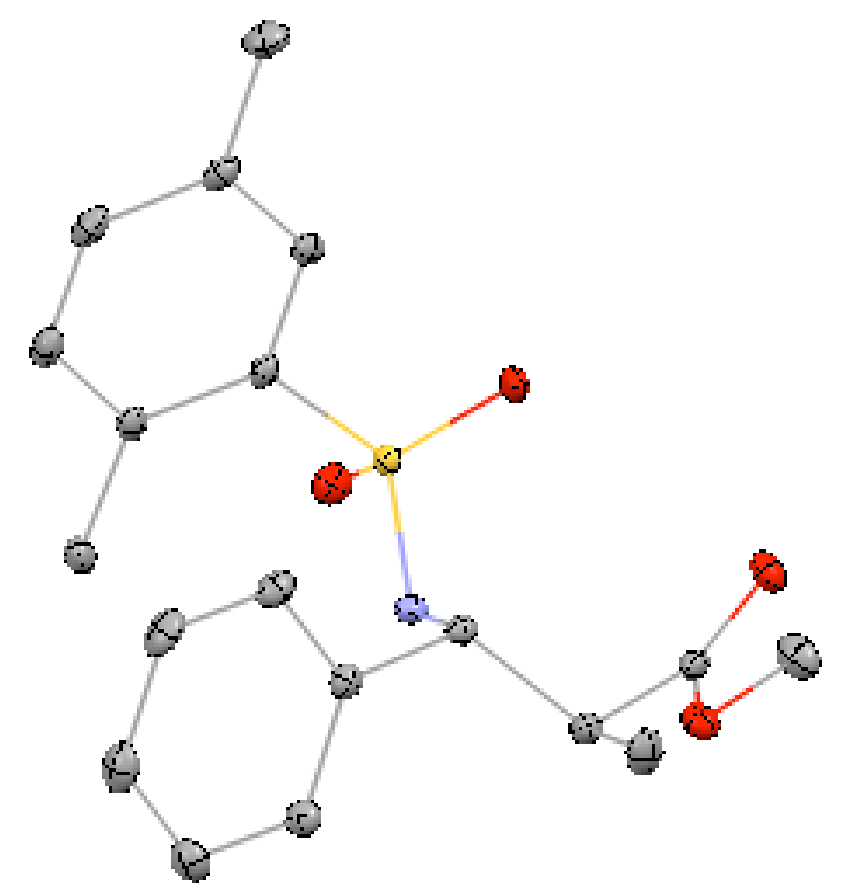

13

\section{A. Crystal Data}

Empirical Formula

Formula Weight

Crystal Color, Habit

Crystal Dimensions

Crystal System

Lattice Type

Indexing Images

Detector Position

Pixel Size

Lattice Parameters
$\mathrm{C}_{19} \mathrm{H}_{23} \mathrm{NO}_{4} \mathrm{~S}$

361.45

colorless, block

$0.60 \times 0.60 \times 0.30 \mathrm{~mm}$

monoclinic

Primitive

3 oscillations @ 90.0 seconds

$127.40 \mathrm{~mm}$

$0.100 \mathrm{~mm}$

$$
\begin{aligned}
& \mathrm{a}=9.5765(3) \AA \\
& \mathrm{b}=9.6154(3) \AA
\end{aligned}
$$




$$
\begin{aligned}
& C=19.9940(7) \AA \\
& \beta=100.6976(12) \circ \\
& V=1809.08(10) \AA^{3}
\end{aligned}
$$

Space Group

$Z$ value

Dcalc

F000

$\mu(\mathrm{MoK} \alpha)$
P21/c (\#14)

4

$1.327 \mathrm{~g} / \mathrm{cm}^{3}$

768.00

$2.020 \mathrm{~cm}^{-1}$ 
B. Intensity Measurements

Diffractometer

Radiation

Detector Aperture

Data Images

$\omega$ oscillation Range $(\chi=45.0, \phi=0.0)$

Exposure Rate

$\omega$ oscillation Range $(\chi=45.0, \phi=180.0)$

Exposure Rate

Detector Position

Pixel Size

$2 \theta \max$

No. of Reflections Measured

Corrections
Rigaku RAXIS-RAPID

$\operatorname{MoK} \alpha(\lambda=0.71075 \AA)$

graphite monochromated

$280 \mathrm{~mm} \times 256 \mathrm{~mm}$

44 exposures

$130.0-190.0^{\circ}$

$30.0 \mathrm{sec} . / 0$

$0.0-160.0^{\circ}$

$30.0 \mathrm{sec} . / \mathrm{O}$

$127.40 \mathrm{~mm}$

$0.100 \mathrm{~mm}$

$54.8^{\circ}$

Total: 17274

Unique: $4124($ Rint $=0.017)$

Lorentz-polarization

Absorption

(trans. factors: $0.788-0.941$ ) 
C. Structure Solution and Refinement

Structure Solution

Refinement

Function Minimized

Least Squares Weights

$\left.4 \mathrm{Fo}^{2}\right)$

$2 \theta$ max cutoff

Anomalous Dispersion

No. Observations (All reflections)

No. Variables

Reflection/Parameter Ratio

Residuals: R1 (I>2.00\%(I))

Residuals: $\mathrm{R}$ (All reflections)

Residuals: wR2 (All reflections)

Goodness of Fit Indicator

Max Shift/Error in Final Cycle

Maximum peak in Final Diff. Map

Minimum peak in Final Diff. Map
Direct Methods

Full-matrix least-squares on $\mathrm{F}^{2}$

$\Sigma w\left(\mathrm{Fo}^{2}-\mathrm{Fc}^{2}\right)^{2}$

$1 /\left[0.0016 \mathrm{Fo}^{2}+1.0000 \mathrm{~s}\left(\mathrm{Fo}^{2}\right)\right] /($

$54.8^{0}$

All non-hydrogen atoms

4124

318

12.97

0.0287

0.0315

0.0970

1.004

0.002

0.35 e/Å3

$-0.42 \mathrm{e} / \AA^{3}$ 


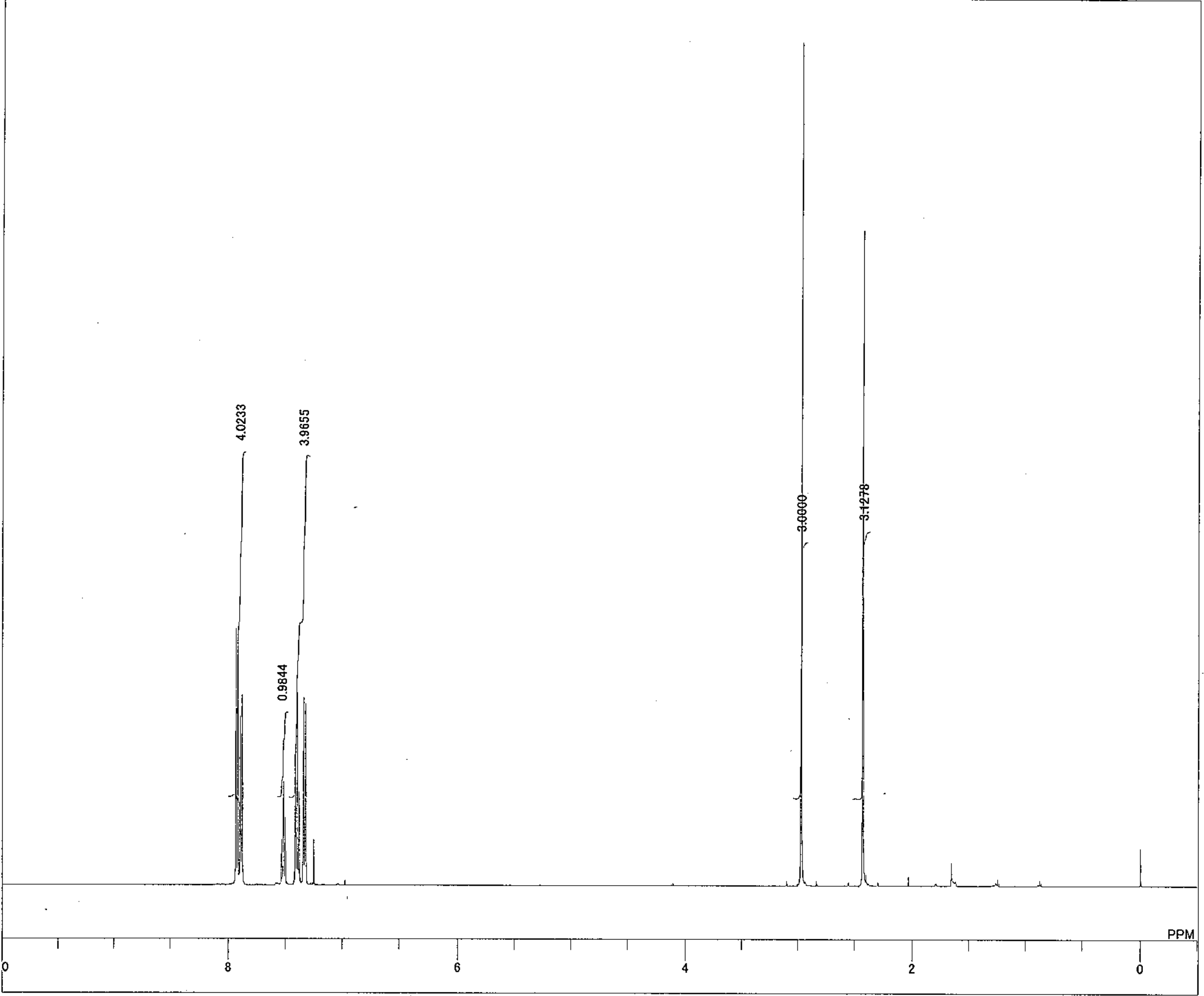

DFILE

COMN

DATIM

OBNUC

OBFRQ

OBSET

POINT

FREQU

SCANS

PD

PW1

CTEMP

EXREF

RFAIN

Documents and Settings $¥ A \mid l$ Users $¥$ Docu 0-08-2007 21:11:17

$1 \mathrm{H}$ NMR.ex2

$495.13 \mathrm{MHz}$

$4.38 \mathrm{KHz}$
$9.64 \mathrm{~Hz}$ 13107

$7429.31 \mathrm{~Hz}$

${ }^{8}$

$5.0000 \mathrm{sec}$

6.50 usec

$29.2 \mathrm{c}$

$0.00 \mathrm{ppm}$
$0.12 \mathrm{~Hz}$

$36 \mathrm{~Hz}$

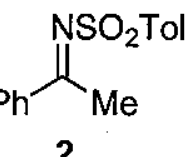




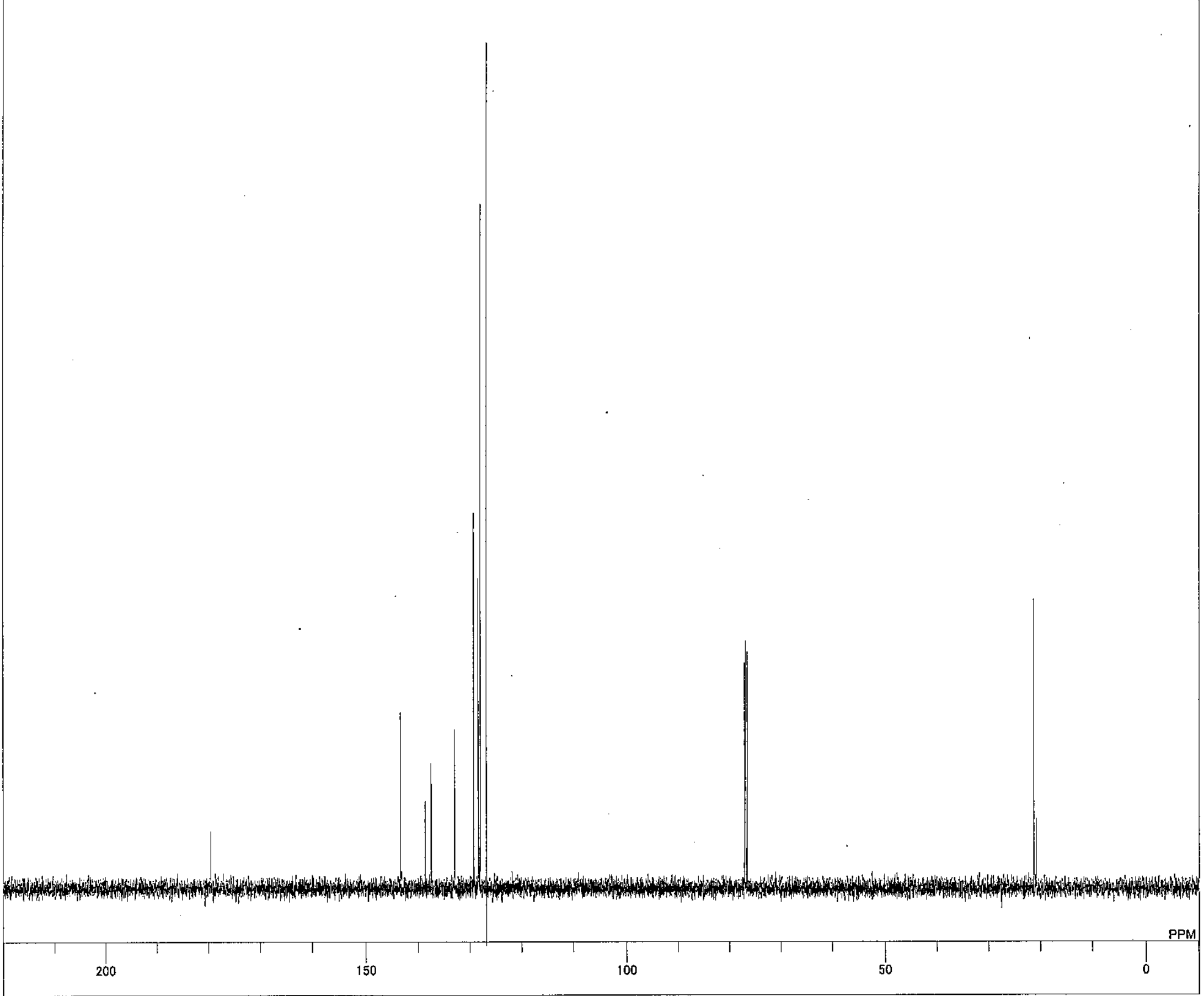

DFLE

EBNuC

EXMOO

OBSET

OBFIN

POINT

FREQU

ACQTM
PD

$\mathrm{PD} 1$

PW1

SLYNT

SLVNT
EXREF
BF

RGAIN

Documents and Settings $¥ A$ All Users $¥$ Docu -08-2007 21:15:54

${ }_{13 \mathrm{C}}$ NMR.ex2 $124.51 \mathrm{MHz}$
$3.45 \mathrm{KHz}$ $6.00 \mathrm{~Hz}$ 26214
$31249.52 \mathrm{~Hz}$ 83 $0.8389 \mathrm{sec}$ $2.0000 \mathrm{sec}$ $1 \mathrm{H} \quad 3.67$ usec $\mathrm{CDCL}^{2} 3.4$ $77.00 \mathrm{ppm}$
$0.12 \mathrm{~Hz}$ 54

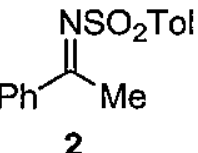


C:FDocuments and Settings $¥ A$ All Users $¥$ Documents $¥$ Matsubara $¥ 2501-2600 * 2600-$ data-1.als

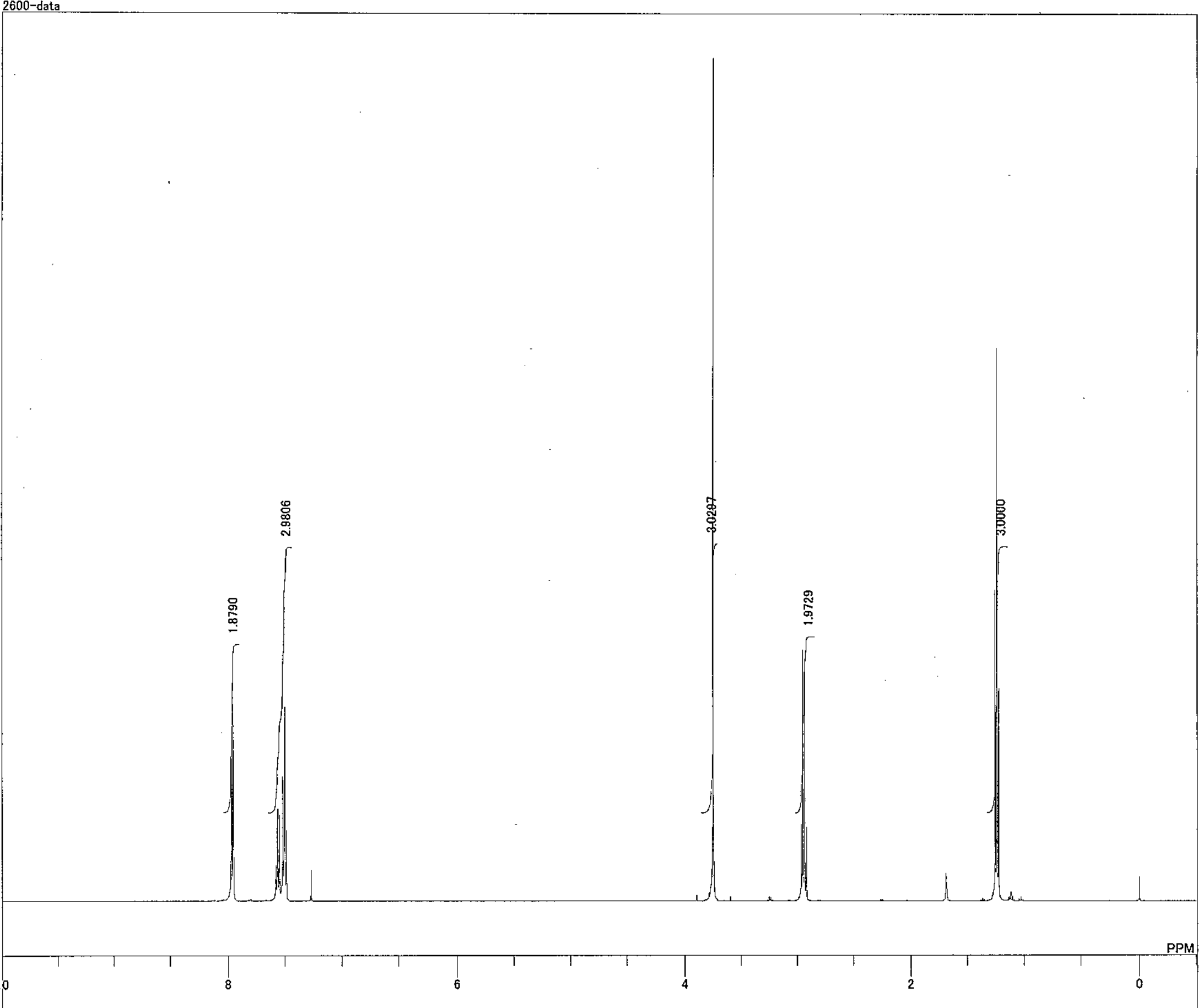

DFILE

COMNT

DBNUC

EXMOD

OBSET

OBFIN

SCANS

CQTM

PD

PW1

CTEMP

SLVNT

EXREF

BF

Documents and Settings:All Users¥Docu 10-08-2007 21:19:21

$1 \mathrm{H}$

$495.13 \mathrm{MHz}$
$4.38 \mathrm{KHz}$

$9.64 \mathrm{~Hz}$

13107

8

$1.7642 \mathrm{se}$

$5.0000 \mathrm{sec}$

$1 \mathrm{H} \quad 6.50$ usec

$\operatorname{CDCL}^{29.2}$

$0.00 \mathrm{ppm}$

$0.12 \mathrm{~Hz}$

$\mathrm{PhO}_{2} \mathrm{~S}$

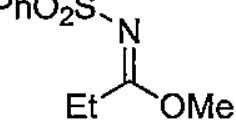

$6 a$ 
C:FDocuments and Settings $¥ A$ All Users $¥$ Doctuments $¥$ Matsubara $¥ 2501-2600 ¥ 2600$-data_C-1.als

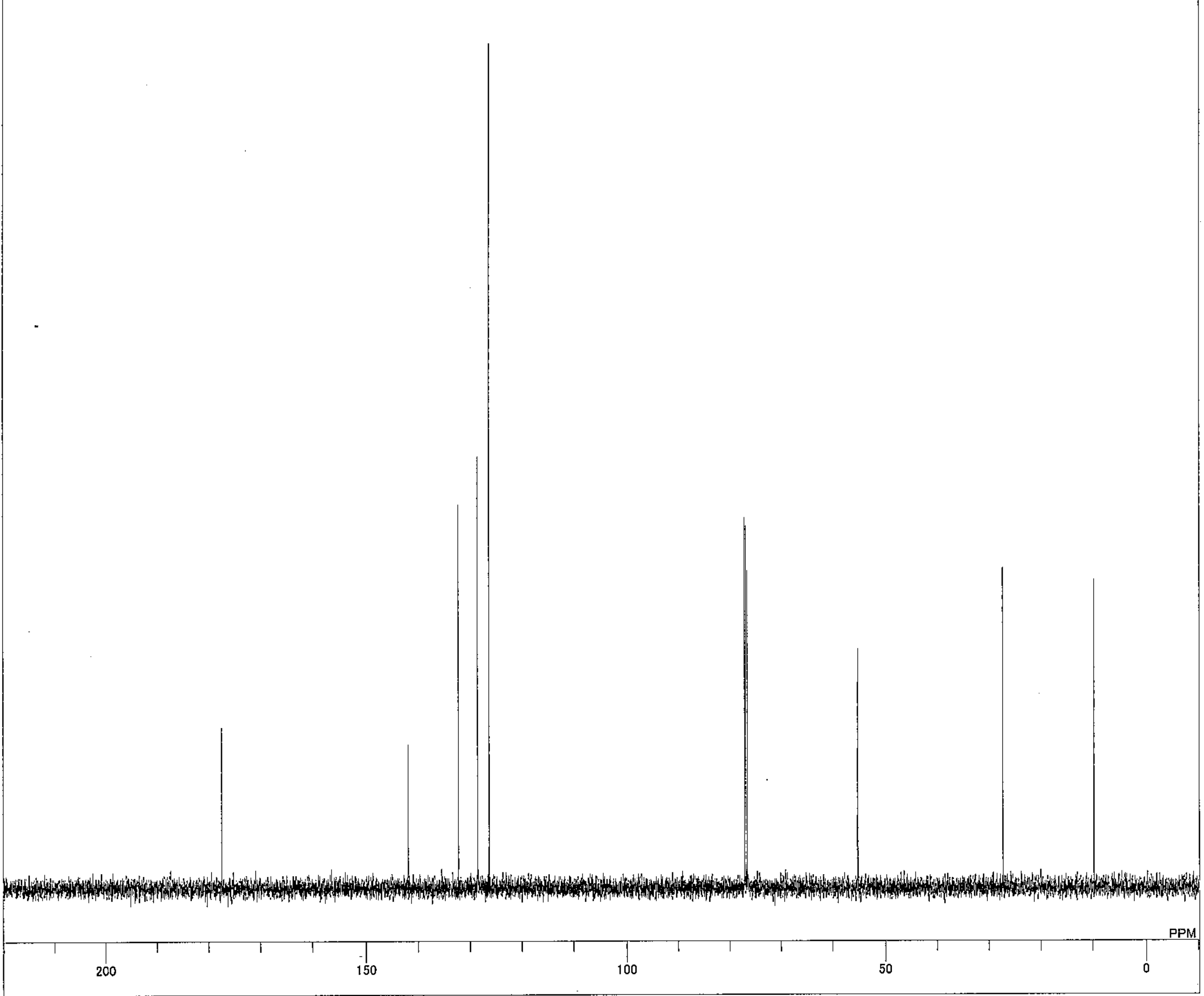

DFILE

EXMOD

OBSET

OBFIN

促

FREQU

ACQTM

$\mathrm{PW} 1$

IRNUC

CTEMP

EXREF

BF 


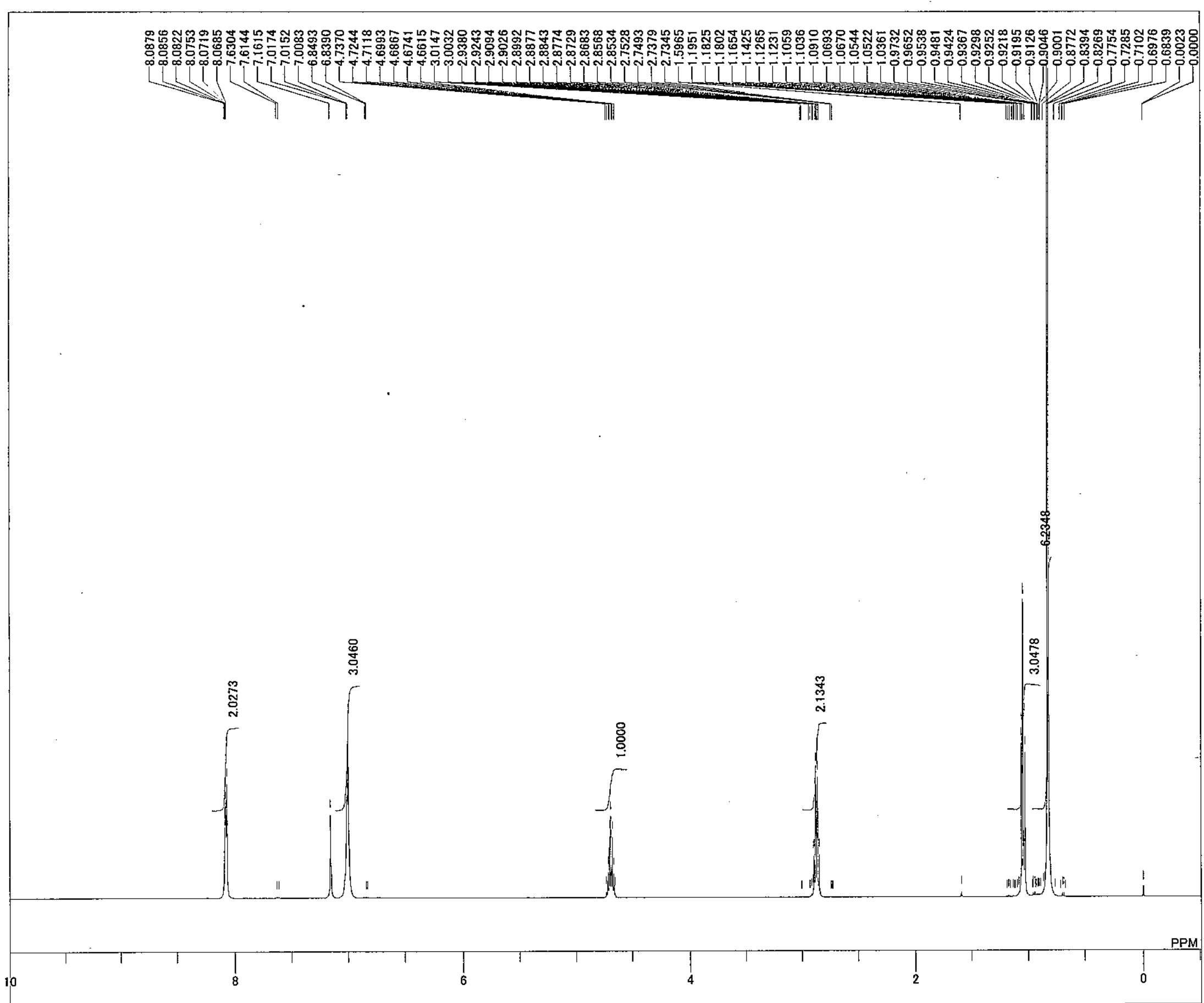

SCANS

ACQTM

$P D$

PW1

CTEMP

SLVNT

EXREF

RGAIN

H NMR.ex2

$495.13 \mathrm{MHz}$

$4.38 \mathrm{KHz}$

$9.64 \mathrm{~Hz}$

$9286.78 \mathrm{~Hz}$

8
$1.7642 \mathrm{sec}$

$5.0000 \mathrm{sec}$

iH $24.4 \mathrm{c}$

C6D6

$0.00 \mathrm{ppm}$
$0.12 \mathrm{~Hz}$
30

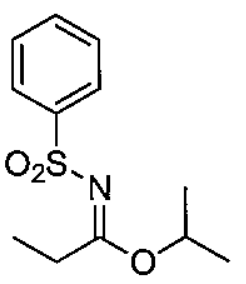

$6 b$ 
C: $:$ Documents and Settings $¥ A$ Ill Users $¥$ Documents $¥$ florian $¥ 500 \mathrm{MH} z ¥ \mathrm{fb} 331 \mathrm{c} 13 \mathrm{pp}-1$.als

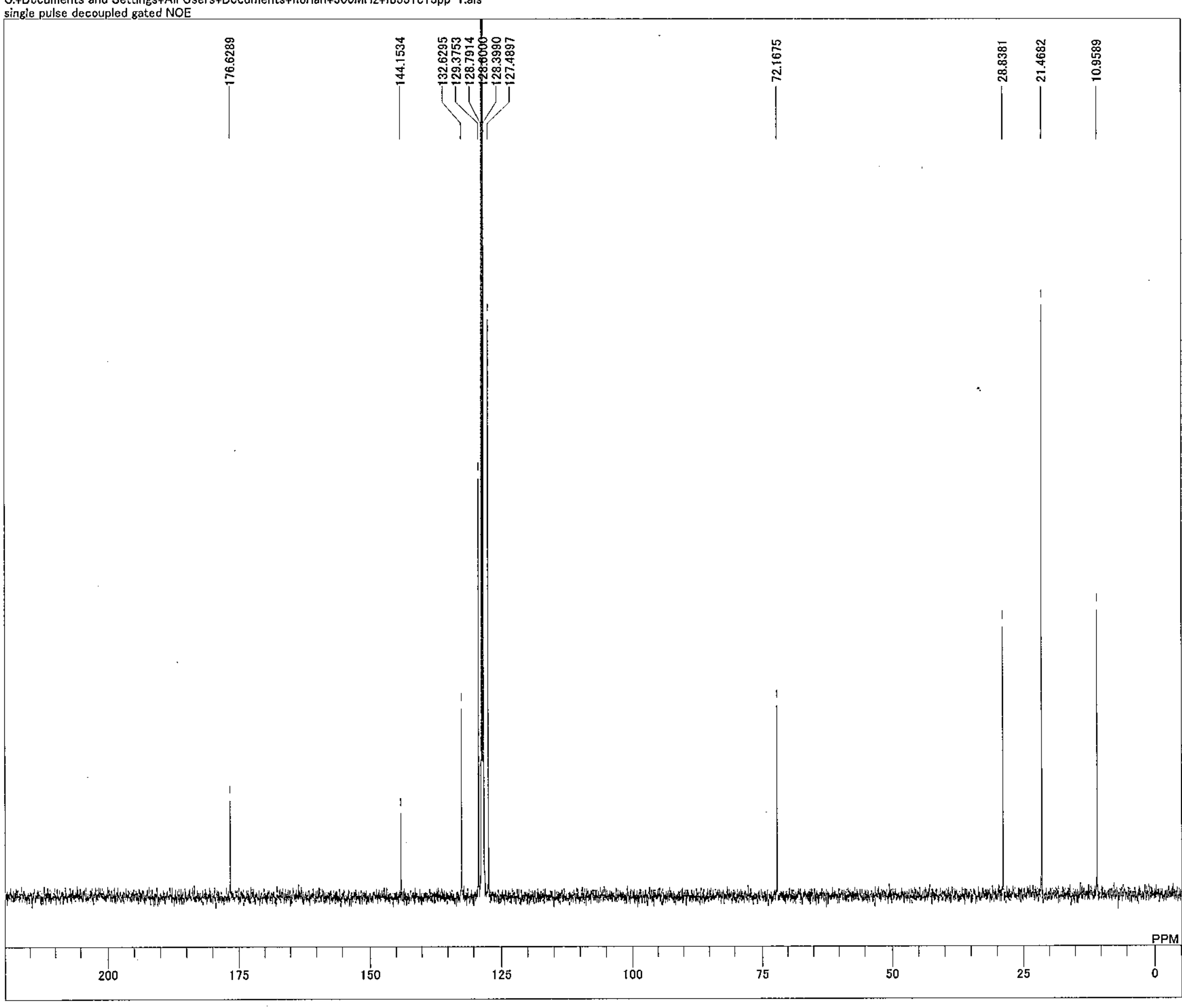

DFILE $\quad$ C: : Documents and Settings:All Users¥Docu

COMNT single pulse decoupled gated NOE

$13 \mathrm{C}$

$124.51 \mathrm{MHz}$

OBSET $\quad 3.45 \mathrm{KHz}$

$31249.52 \mathrm{~Hz}$

$\begin{array}{lc}\text { SCANS } & 62 \\ \text { ACQTM } & 0.8389 \mathrm{sec}\end{array}$

$\begin{array}{ll}\mathrm{ACQTM} & 0.8289 \mathrm{sec} \\ \mathrm{PD} & 2.0000 \mathrm{sec}\end{array}$

IRNUC $1 \mathrm{H} 3.67$ useo

CTEMP $24.7 \mathrm{c}$

$\begin{array}{ll}\text { SLVNT } & \text { C6D6 } \\ \text { EXREF } & 128.60\end{array}$

$\begin{array}{lc}\text { EXREF } & 128.60 \mathrm{ppm} \\ \text { BF } & 0.12 \mathrm{~Hz} \\ \text { RGAIN } & 50\end{array}$

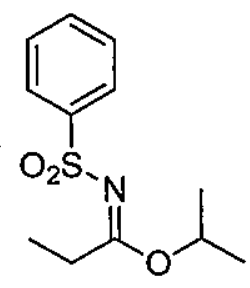

$6 b$ 


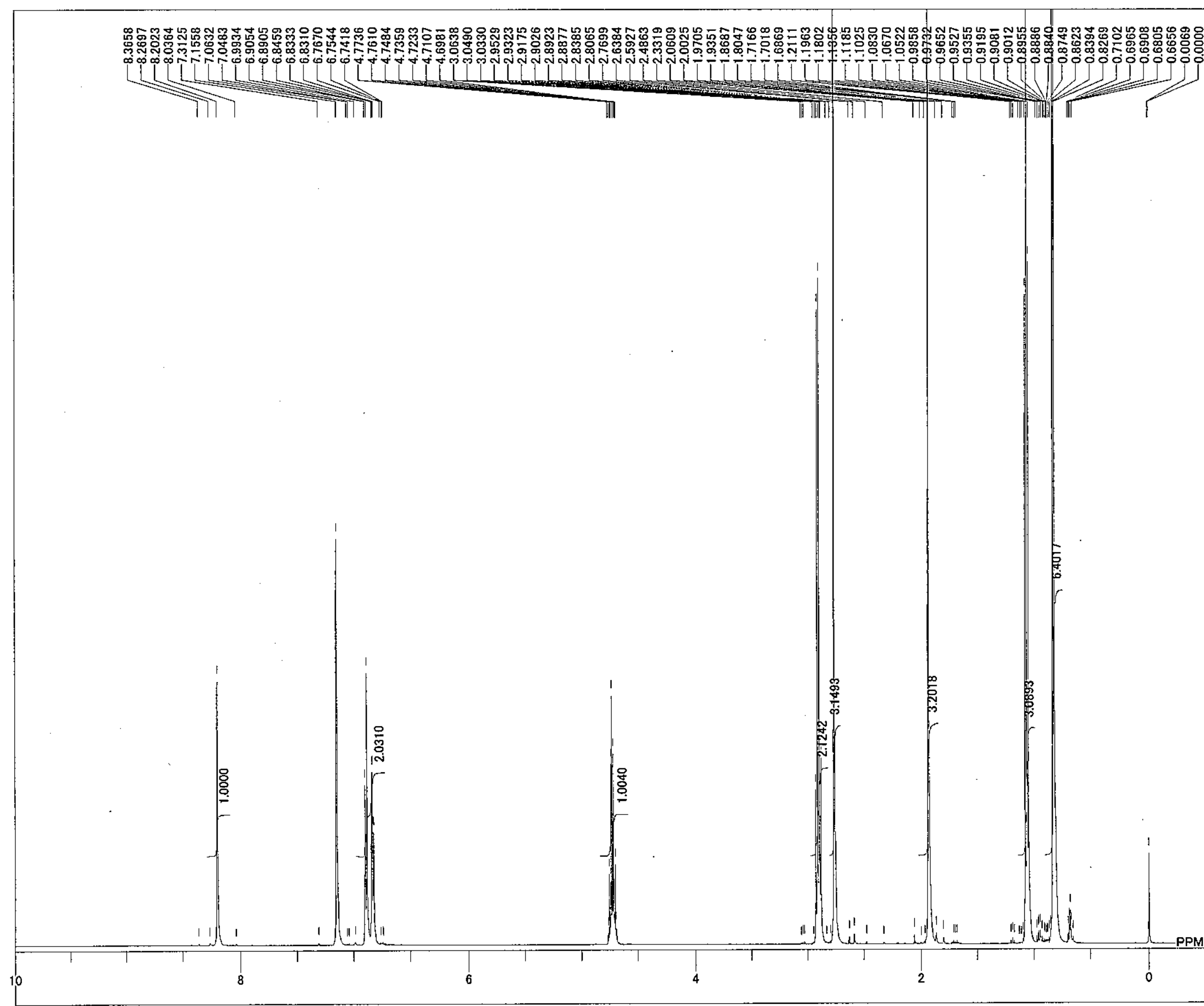

DFILE $\quad$ $¥ E \mathrm{E}$ ca $¥$ data $¥$ florian $¥ \mathrm{fb} 360 \mathrm{pp}-1, \mathrm{jdf}$

NMR.ex2
$495.13 \mathrm{MHz}$ $4.38 \mathrm{KHz}$ $9.64 \mathrm{~Hz}$ $9286.78 \mathrm{~Hz}$

8
$1.7642 \mathrm{sec}$ $1.7642 \mathrm{sec}$
$5.0000 \mathrm{sec}$

C $6 \mathrm{D} 6{ }^{24.5 \mathrm{c}}$

$0.00 \mathrm{ppm}$ ${ }_{34}^{0.12 \mathrm{~Hz}}$

BF

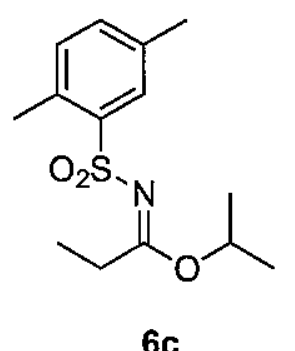

6c 
C:FDocuments and Settings $¥$ All Users $¥$ Documents $¥$ florian $¥ 500 \mathrm{MHz} ¥ \mathrm{fb} 360 \mathrm{c} 13 \mathrm{pp}-1$. als

single pulse decoupled gateo NOE
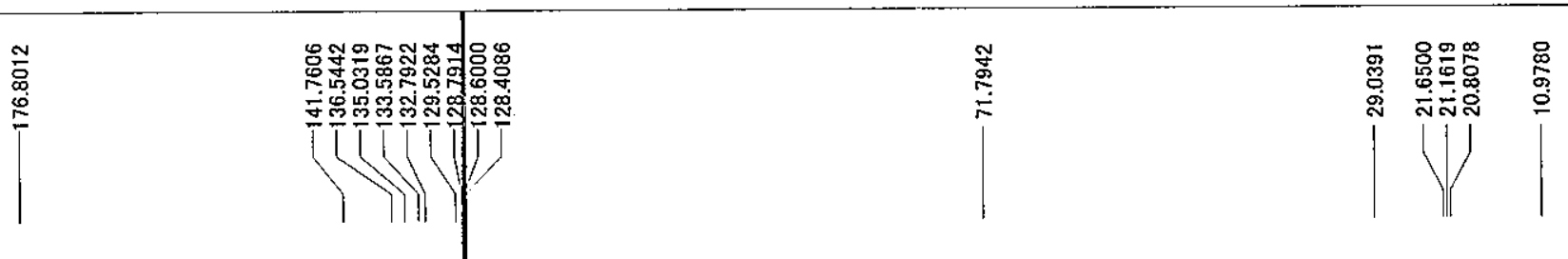

OBNUC

EXMOD

OBFRQ

OBFIN

POINT

SCANS

ACQTM

PW1

IRNUC

CTEMP

EXREF

EXREF

Documents and Settings¥All Users $¥$ Docu gle pulse decoupled gated NOE

10-07-2007 18:08:03

13C NMR.ex2 $124.51 \mathrm{MHz}$
$3.45 \mathrm{KHz}$ $6.00 \mathrm{~Hz}$ 26224 $31249.52 \mathrm{~Hz}$ 62
$0.8389 \mathrm{sec}$ $2.0000 \mathrm{sec}$
$3.67 \mathrm{usec}$ H ${ }^{2} 6 \mathrm{D}{ }^{24.8 \mathrm{c}}$ $128.60 \mathrm{ppm}$
$0.12 \mathrm{~Hz}$ 50

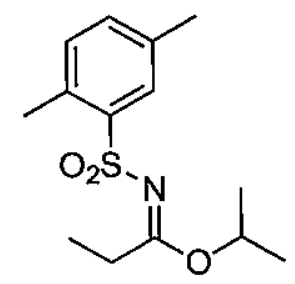

$6 c$

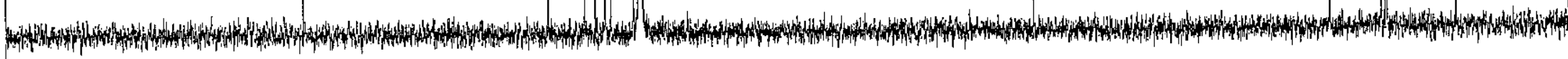

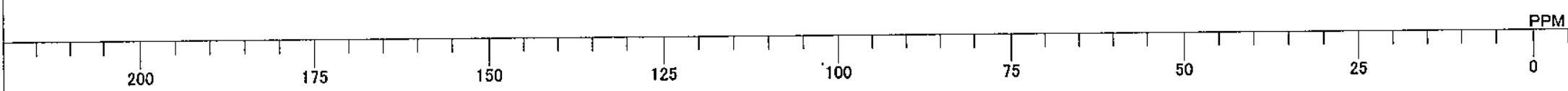


\#\#Eca*data\#florian\#fo366 $\mathrm{pp} 2-1$.jdf

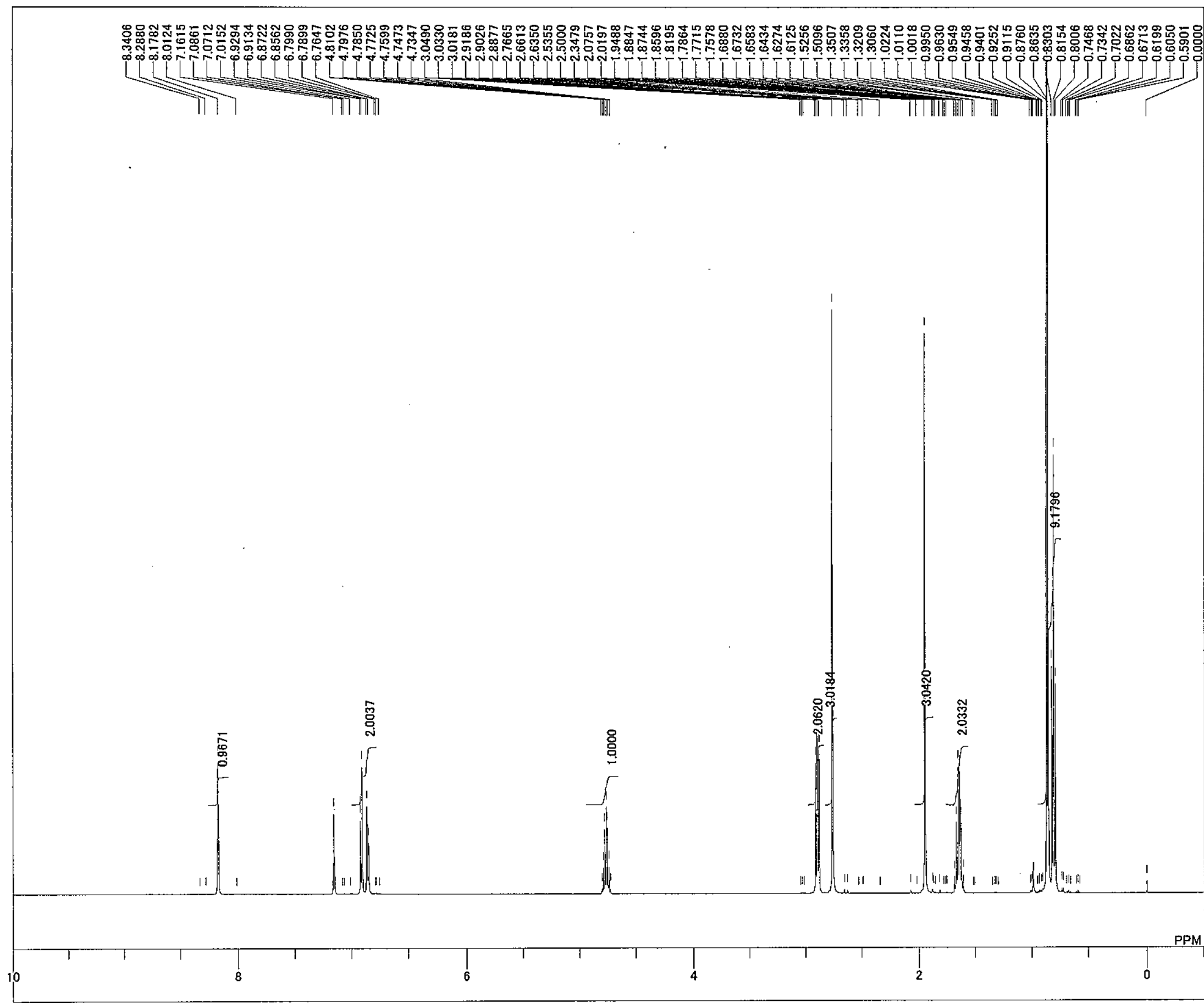

1H NMR.ex2

$495.13 \mathrm{MHz}$
$4.38 \mathrm{KHz}$ $9.64 \mathrm{~Hz}$

$9286.78 \mathrm{~Hz}$

$1.7642 \mathrm{sec}$

$5.0000 \mathrm{sec}$

IH 6.50 usec

$\mathrm{C}^{2} 6^{24.5 \mathrm{c}}$

$0.00 \mathrm{ppm}$
$0.12 \mathrm{~Hz}$
26

BF

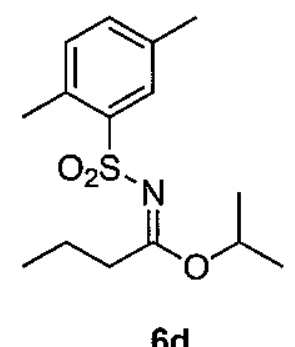

$6 d$ 
C:₹Documents and Settings $¥$ All Users $¥$ Documents $¥$ florian $¥ 500 \mathrm{MHz} ¥ f b 366 \mathrm{c} 13 \mathrm{pp} 2-1$.als

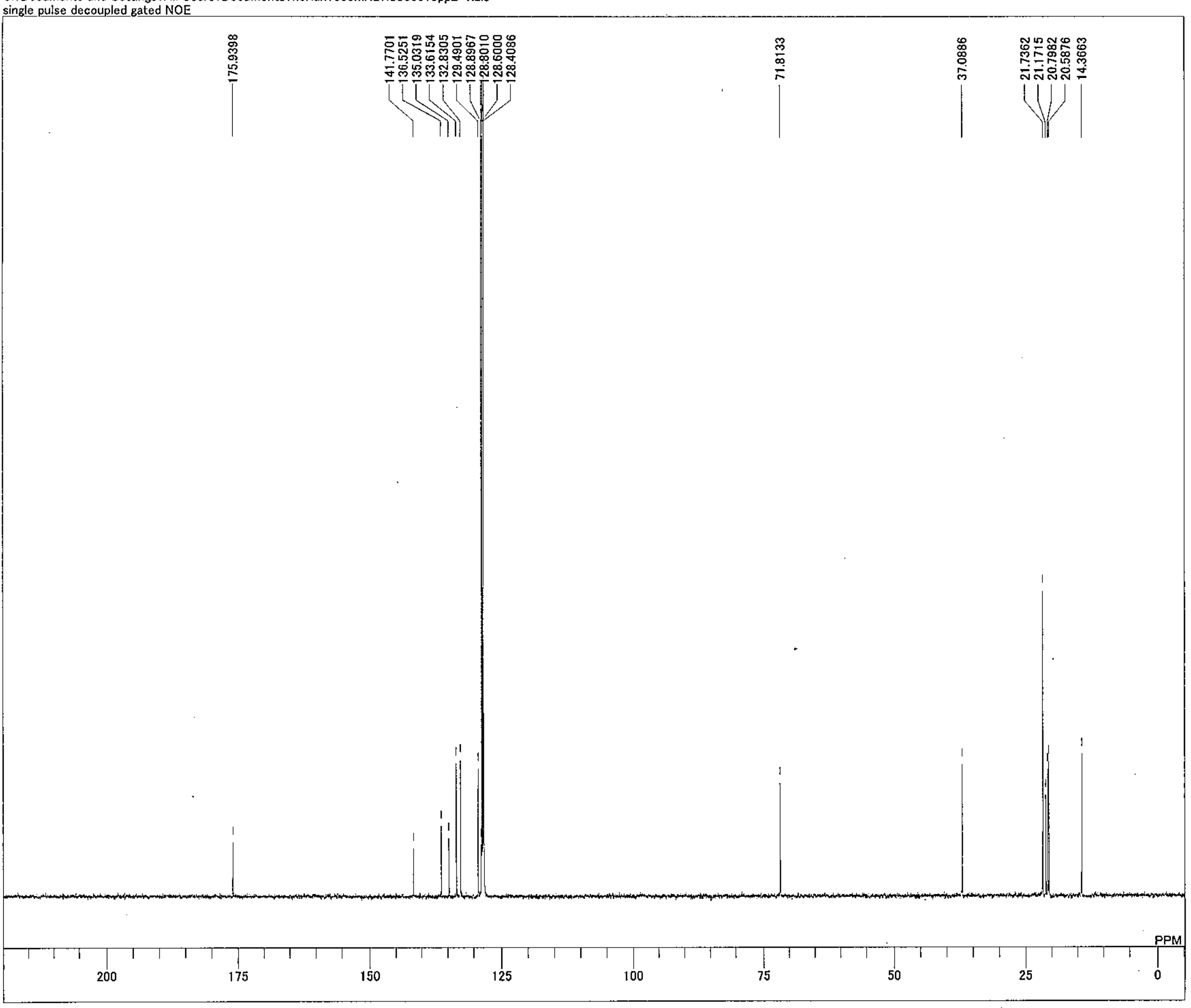

DFILE

COMNT

DATIM

OBNUC

OBSET

OBFIN

POINT

ACQTM

PD

PWIT

CTEMP

SLVNT

EXREF

BF

Users¥Docu .

$13 \mathrm{C}$

$24.51 \mathrm{MHz}$

$3.45 \mathrm{KHz}$

$6.00 \mathrm{~Hz}$

$31249.52 \mathrm{~Hz}$

$0.8389 \mathrm{sec}$

$2.0000 \mathrm{sec}$

$1 \mathrm{H} \quad 3.67 \mathrm{usec}$

$\mathrm{C} 606{ }^{24.9}$

$128.60 \mathrm{ppm}$
$0.12 \mathrm{~Hz}$
50

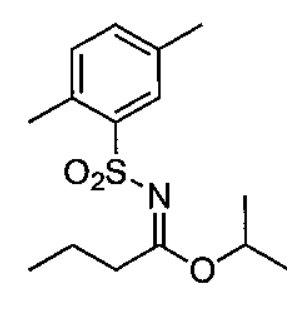

$6 d$ 


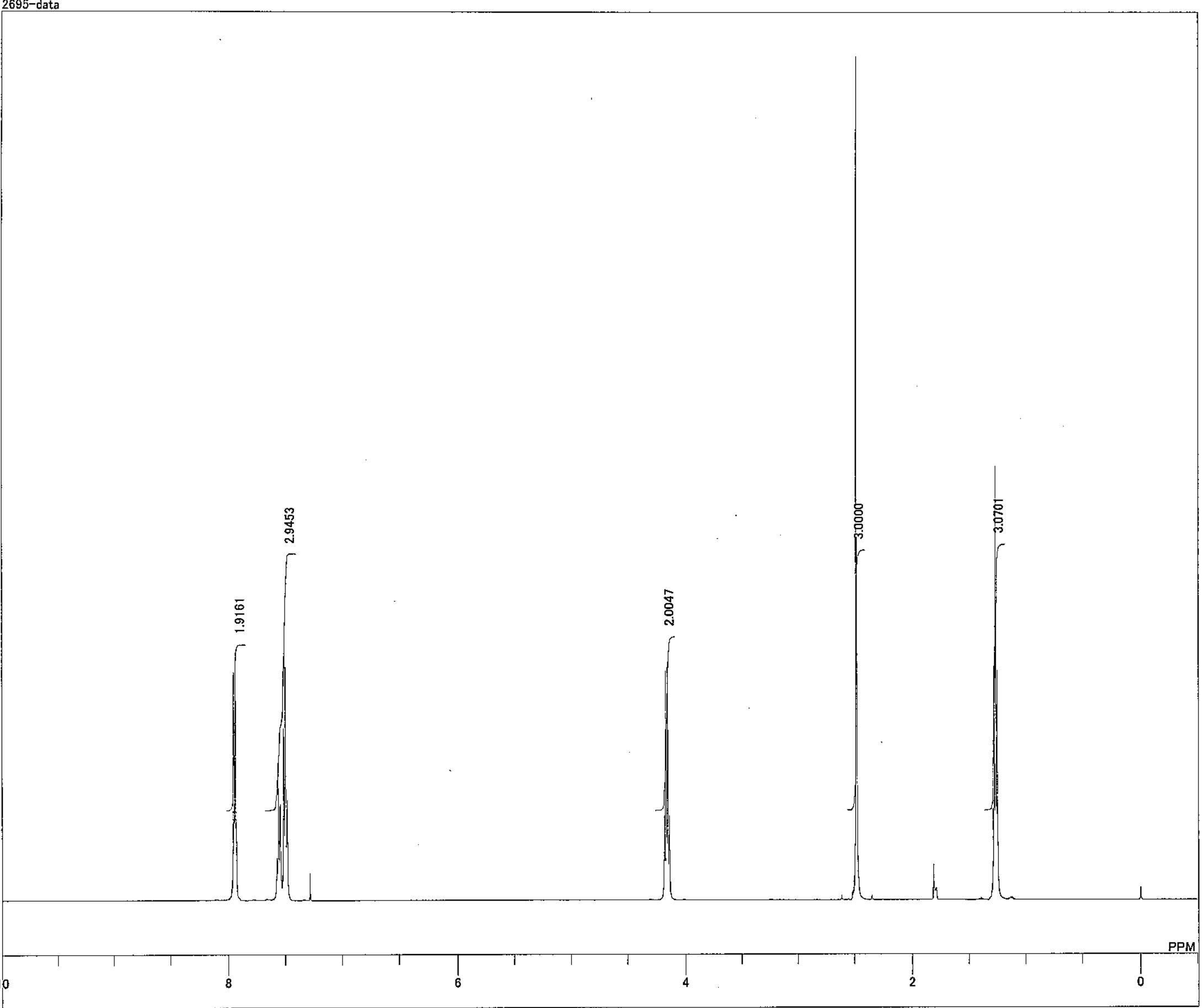

DFILE

COMNT

OBNUC

OBFRQ

OBSEY

OBFIN
POINT

FREQU

SCANS

ACQTM
PD
PW1
IRNUC

IRNuC

CTEMP

EXEEF

RGAIN

Documents and Settings¥All Users¥Docu

10-08-2007 21:29:01

$1 \mathrm{H}$ NMR.ex2

$495.13 \mathrm{MHz}$

$4.38 \mathrm{KHz}$

$9.64 \mathrm{~Hz}$

$7429.31 \mathrm{~Hz}$

8

$.7642 \mathrm{se}$

$5.0000 \mathrm{sec}$

it 6.50 usec

$\mathrm{CDCL}_{3}^{29.3 \circ}$

$0.00 \mathrm{ppm}$
$0.12 \mathrm{~Hz}$
30

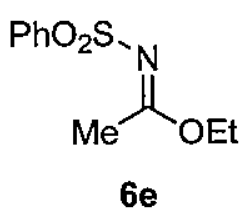


C: $¥$ Documents and Settings $¥$ All Users $¥$ Documents $¥$ Matsubara $¥ 2601-2700 ¥ 2695-$ data-C-1.als 2695-data-

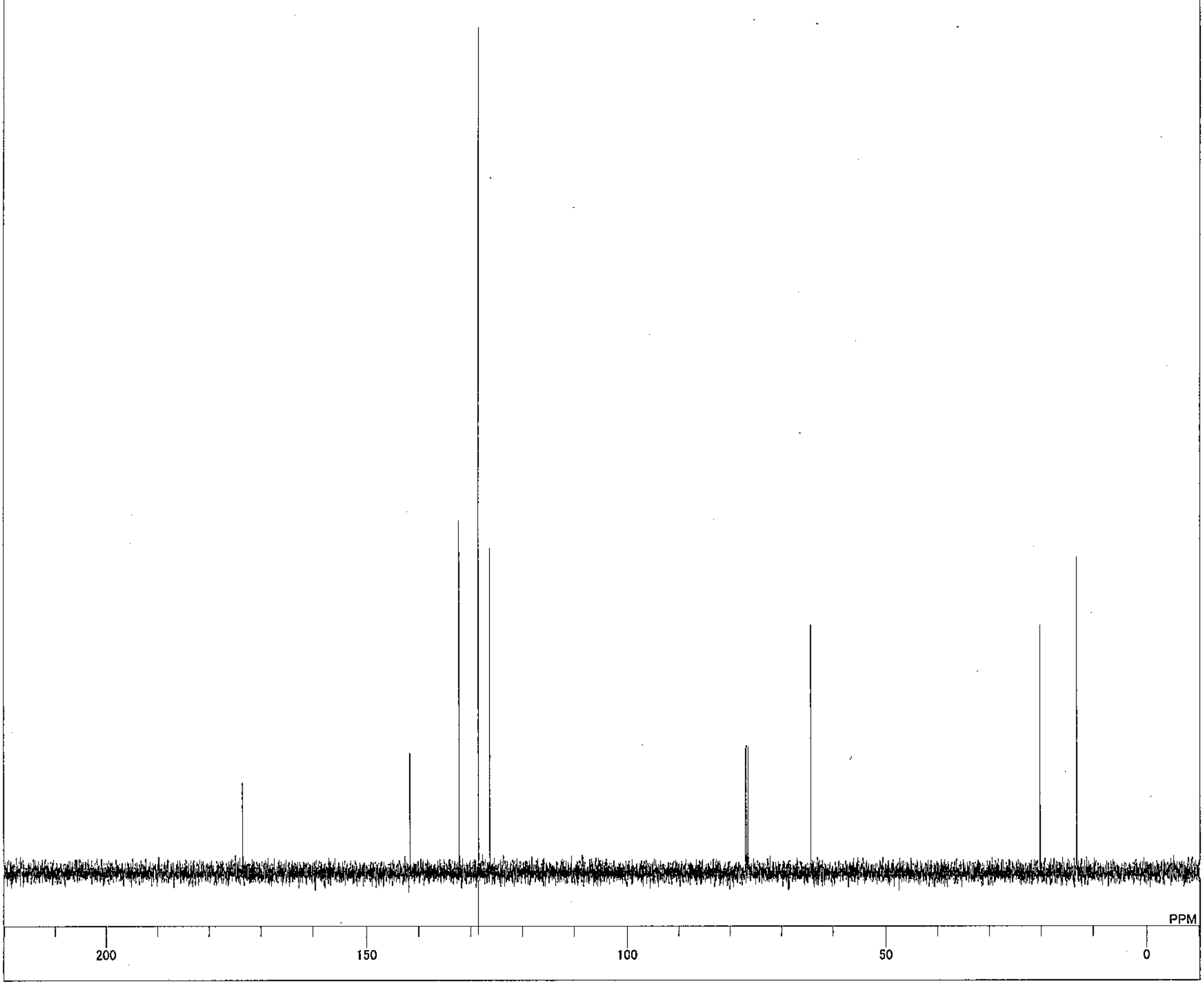

OBFIN

SCANS

ACQTM

PD

IRNUC

CTEMP
SLVNT

SLVNT

BGAIN

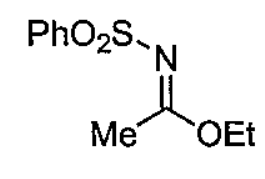

$6 e$ 


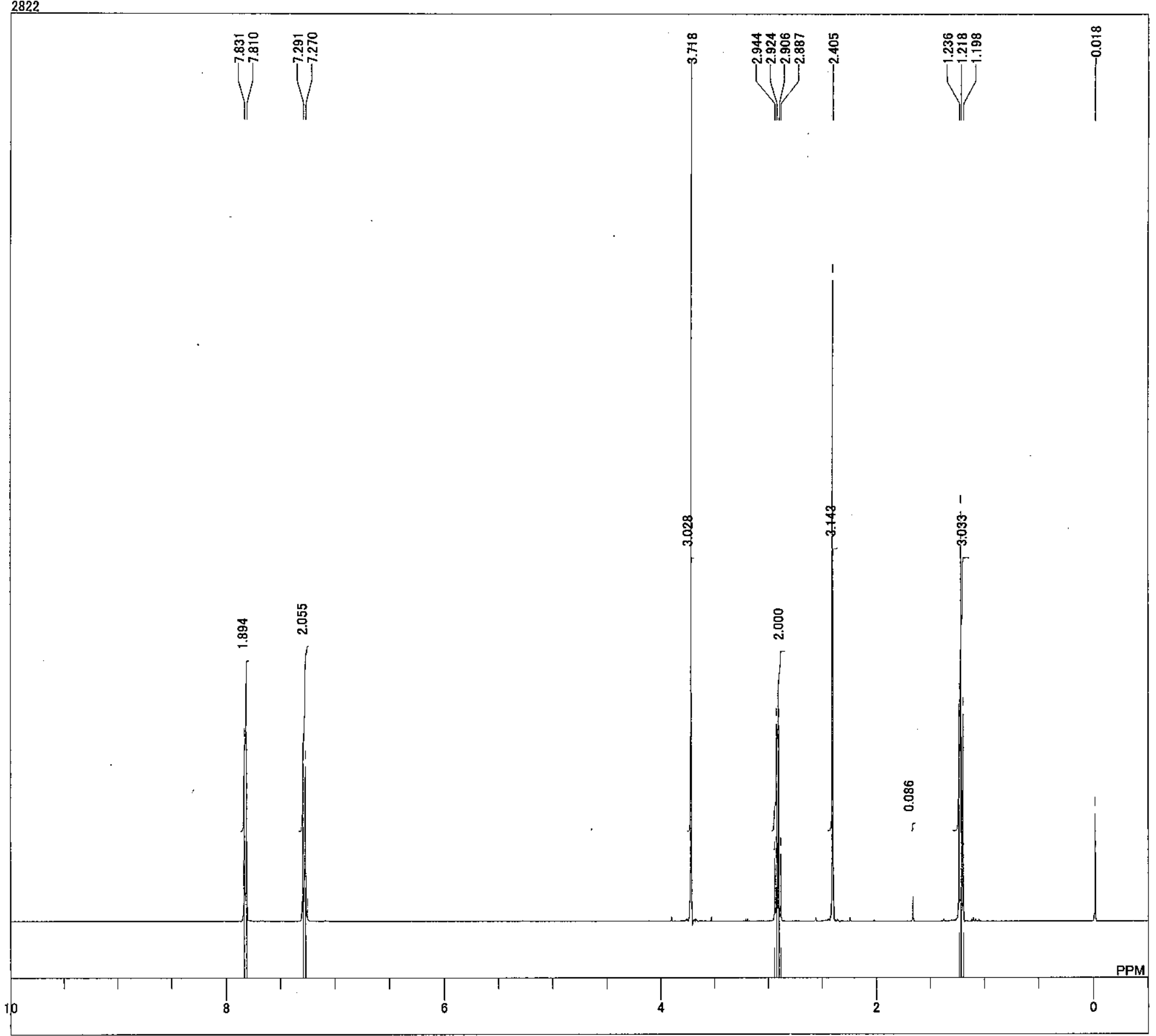

DATIM

OBNUC

EXMOD

OBSET

OBFIN

POINT
FREQU

FREQU

ACQTM

PD

PW1

$25.0 \mathrm{c}$

RGAIN

$12.51 \mathrm{ppm}$ $0.12 \mathrm{~Hz}$
28

$1 \mathrm{H}$-NMR (CDCl3) $\delta$ :

$7.82(2 \mathrm{H}, \mathrm{d}, J=8.2 \mathrm{~Hz})$,

$2.92(2 \mathrm{H}, \mathrm{q}, \mathrm{J}=7.6 \mathrm{~Hz})$,
$1.22(3 \mathrm{H}, \mathrm{t}, \mathrm{J}=7.6 \mathrm{~Hz})$.
Ts $N$

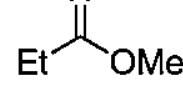

$6 f$ 


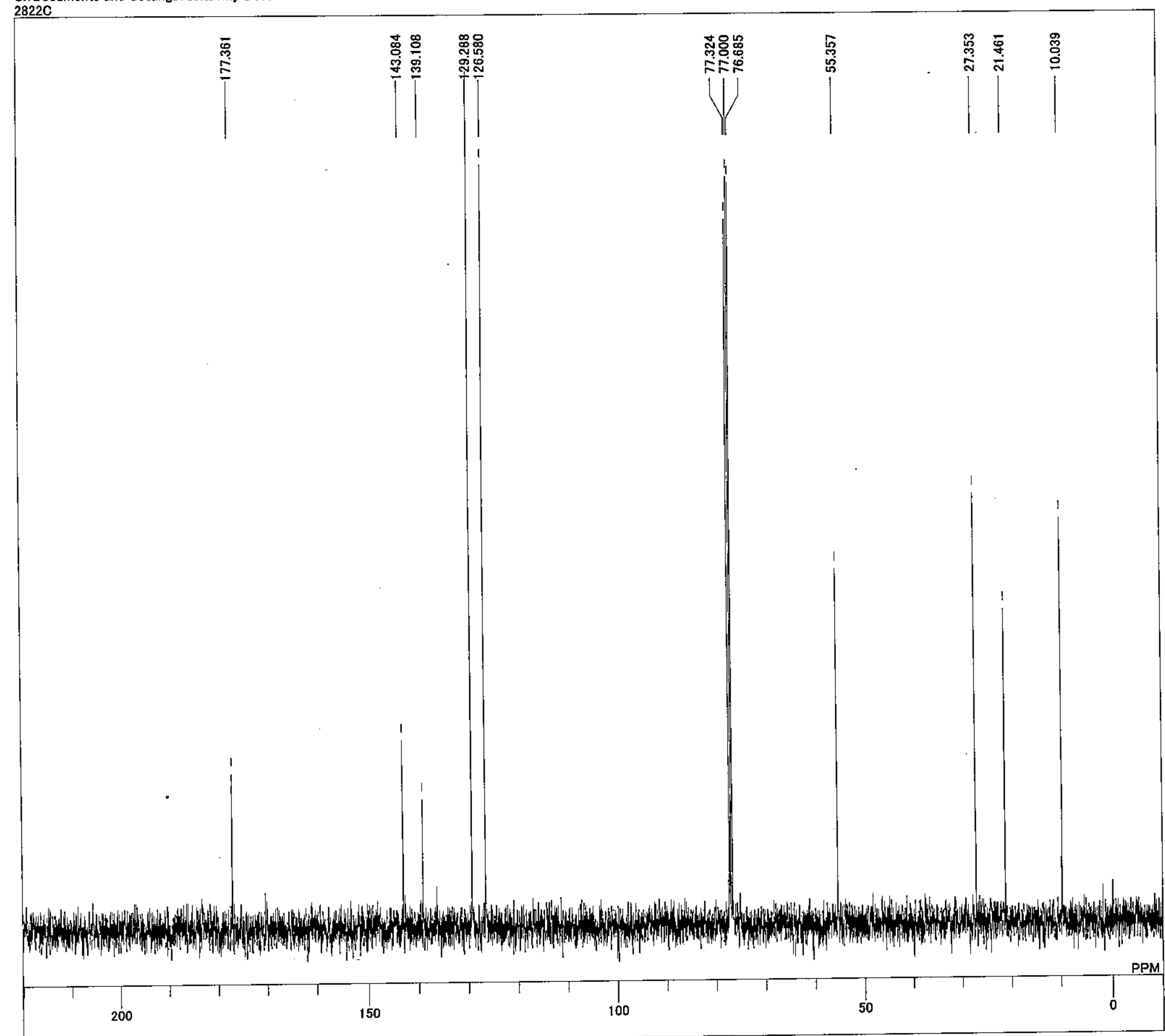

DFILE C:*Documents and Settings*delta*My Documents*Porss:+1

DATIM 2822C 2007151509

OBNUC $13 \mathrm{C}$

EXMOD single.pulse_dec

$\begin{array}{lc}\text { OBFRQ } & 100.53 \mathrm{MHz} \\ \text { OBSET } & 5.35 \mathrm{KHz}\end{array}$

$\begin{array}{ll}\text { OBSET } & 5.35 \mathrm{KHz} \\ \text { OBFIN } & 5.86 \mathrm{~Hz}\end{array}$

POINT $\quad 32768$

SCANS

$\begin{array}{ll}\text { ACQTM } & 1.0433 \mathrm{sec} \\ 2 \mathrm{PD} & 2.0000 \mathrm{sec}\end{array}$

$\begin{array}{lr}\text { PD } & 2.0000 \mathrm{sec} \\ \text { PW1 } & 2.83 \mathrm{usec}\end{array}$

IRNUC $1 \mathrm{H} \quad 2490$

SLVNT CDCL 3.9

$\begin{array}{lc}\text { EXREF } & 77.00 \mathrm{ppm} \\ \text { BF } & 0.12 \mathrm{~Hz} \\ \text { RGAIN } & 50\end{array}$

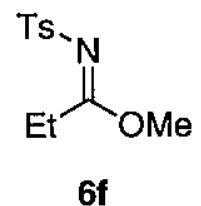


C:FDocuments and Settings¥All Users*Documents¥Matsubara*2801-2900*2805-1.als

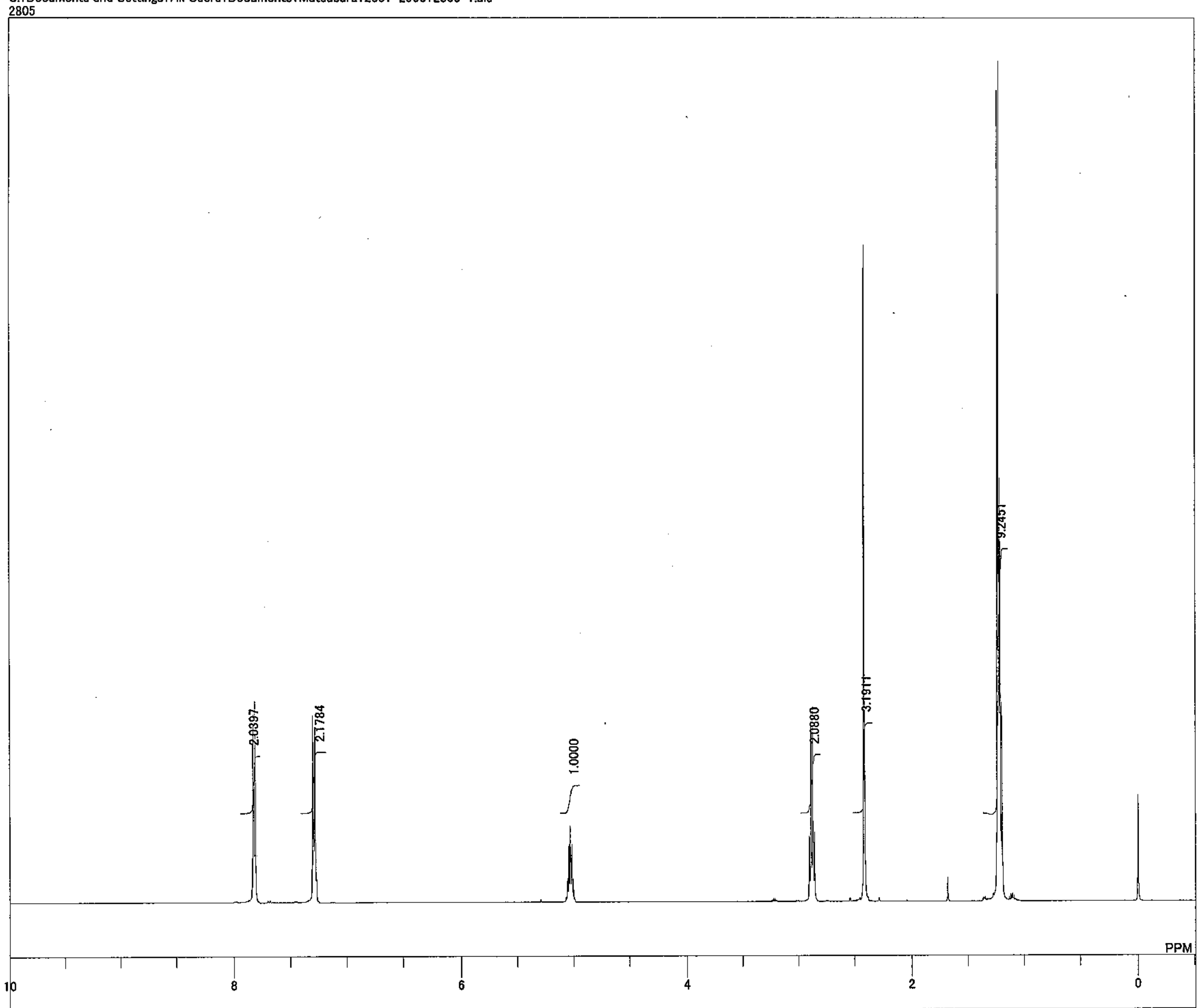

DFILE

COMNT

DATIM

EXMOD

OBFRQ

政

BFIN

PREQU

SCANS

ACQTM

PD

IRNUC

TEMP

BF

Documents and Settings $¥ A$ All Users $¥$ Doc

0-11-2007 20:27:48

H NMR.ex2
$495.13 \mathrm{MH}$

$495.13 \mathrm{MHz}$

$4.38 \mathrm{KHz}$

$9.64 \mathrm{~Hz}$

$7429.31 \mathrm{~Hz}$

8

$1.7642 \mathrm{sec}$

$5.0000 \mathrm{sec}$

IH $21.9 \mathrm{c}$

$\mathrm{CDCL}^{2}$

$0.00 \mathrm{ppm}$
$1.20 \mathrm{~Hz}$

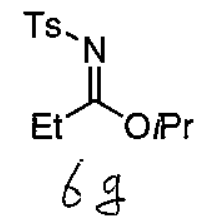




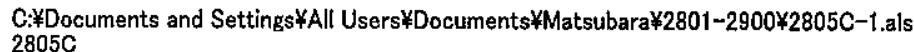

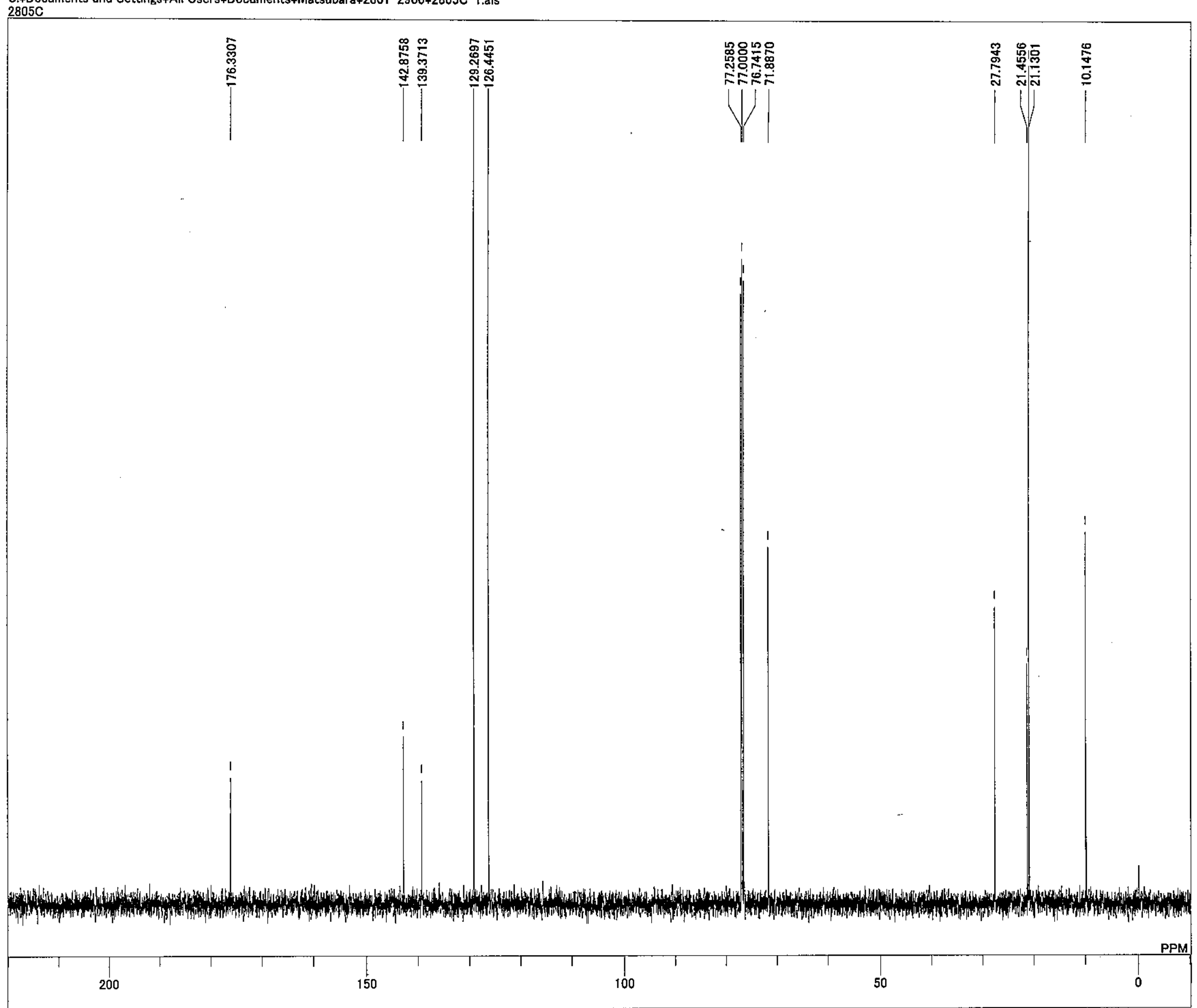

DFILE

DATIM

OBNUC

EXMOD

OBFRQ

OBFIN

FREQU

SCANS

PD

IRNUC

CTEMP

EXREF

RGAIN

$\mathrm{CDCL}^{22.5}$

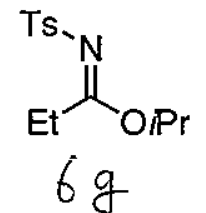

$77.00 \mathrm{ppm}$
$1.20 \mathrm{~Hz}$
50

Documents and

1-2007 20:30:23

NMR.ex2
$124.51 \mathrm{MH}$

$3.45 \mathrm{KHz}$

26214

$0.8389 \mathrm{sec}$

.

$6 \mathrm{~g}$ 


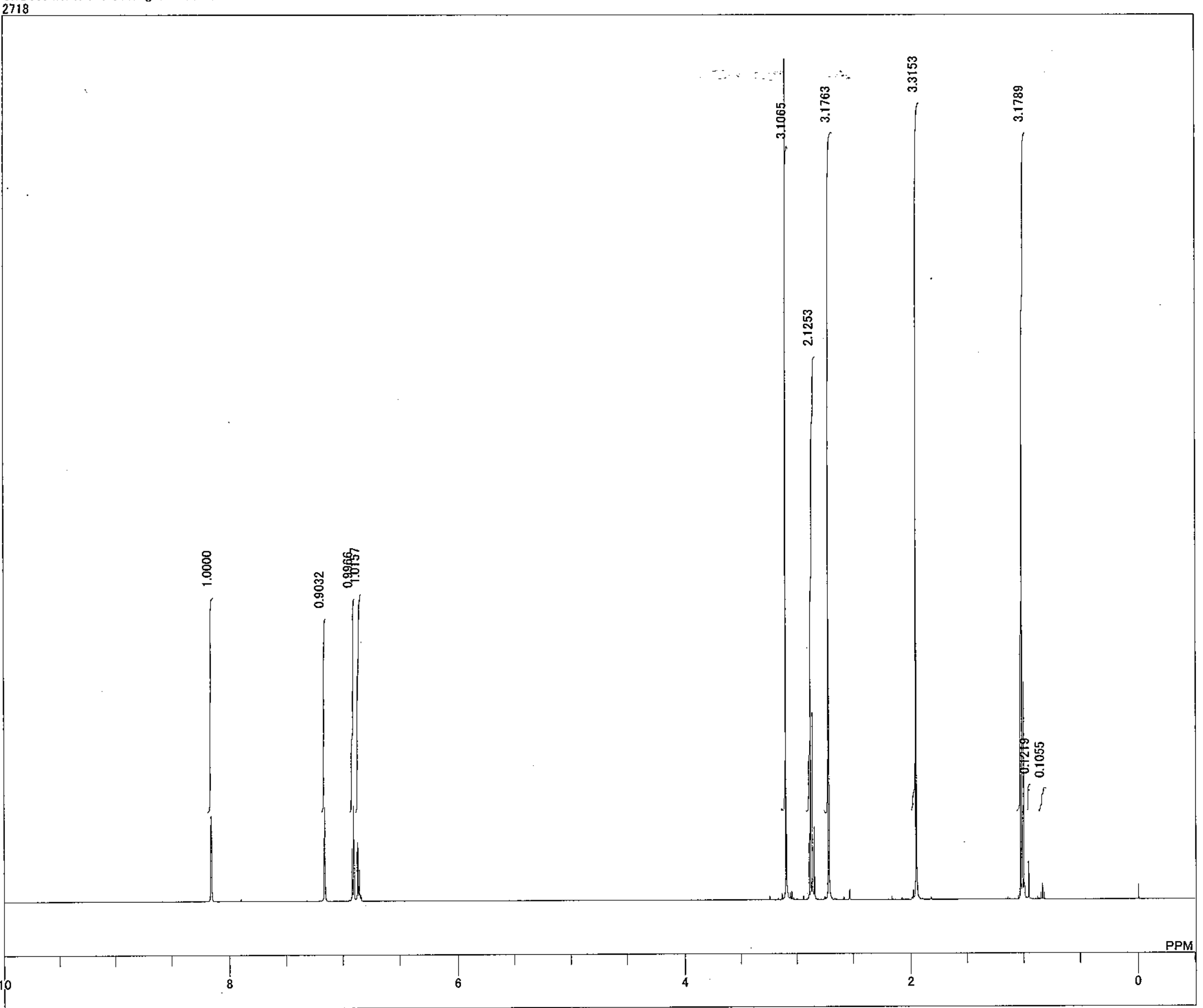

DFILE

.7Documents and Settings $¥ A l l$ Users $¥$ Docu

02-08-2007 09:30:12

OBSET

OBFIN

POINT

SCANS

ACQTM

PD

PW1

CTEMP

SLVNT

EXREF

RGAIN

$0.00 \mathrm{ppm}$

$0.12 \mathrm{~Hz}$

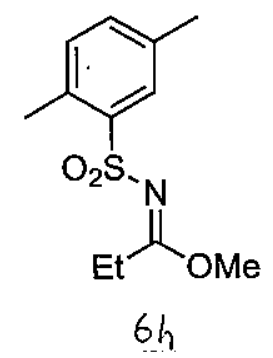


C:¥Documents and Settings¥All Users¥Documents¥Matsubara $¥ 2701-2800 ¥ 2718$ _C-1.als

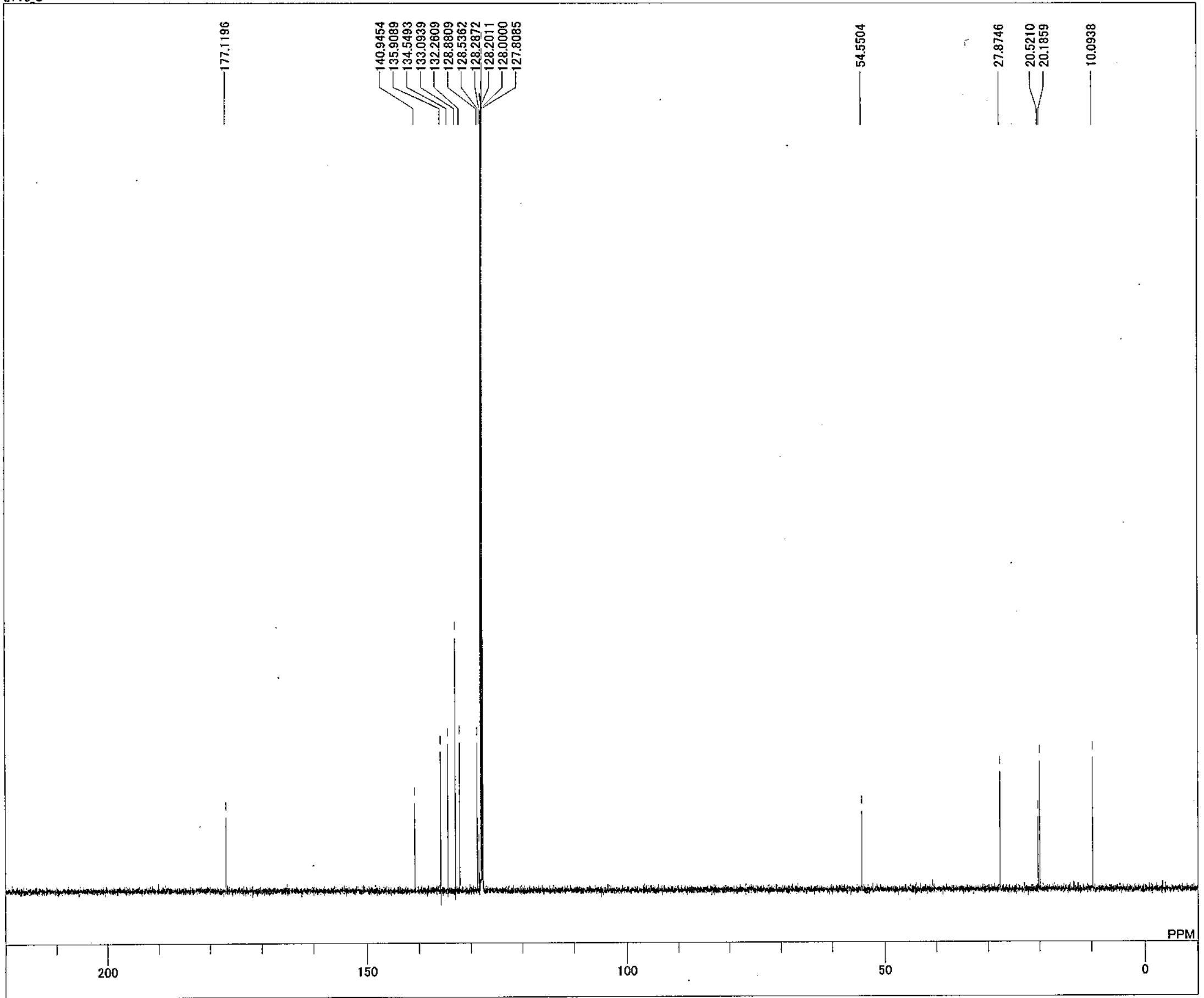

DFILE C: $\quad$ Documents and Settings $¥$ Ail Users $¥$ Docu

COMNT

OBNUC

EXMOD

OBSET

OBFIN

FREQU

ACQTM

PD

IRNUC 1

$-08-2007$ 09:35:51

SC NMR.ex2

$124.51 \mathrm{MHz}$ $3.45 \mathrm{KHz}$ 26214 26214 79 $0.8389 \mathrm{sec}$ $2.0000 \mathrm{sec}$

CTEMP $1 \mathrm{H} 270$ 。

LVNT

EXREF

RG

606

$128.00 \mathrm{ppm}$
$0.12 \mathrm{~Hz}$

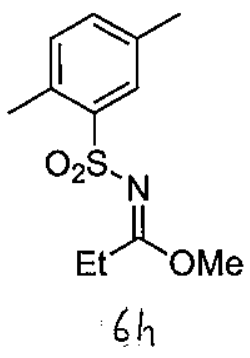

PPM 


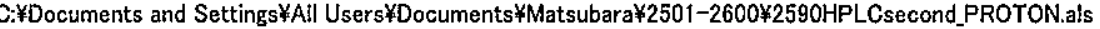

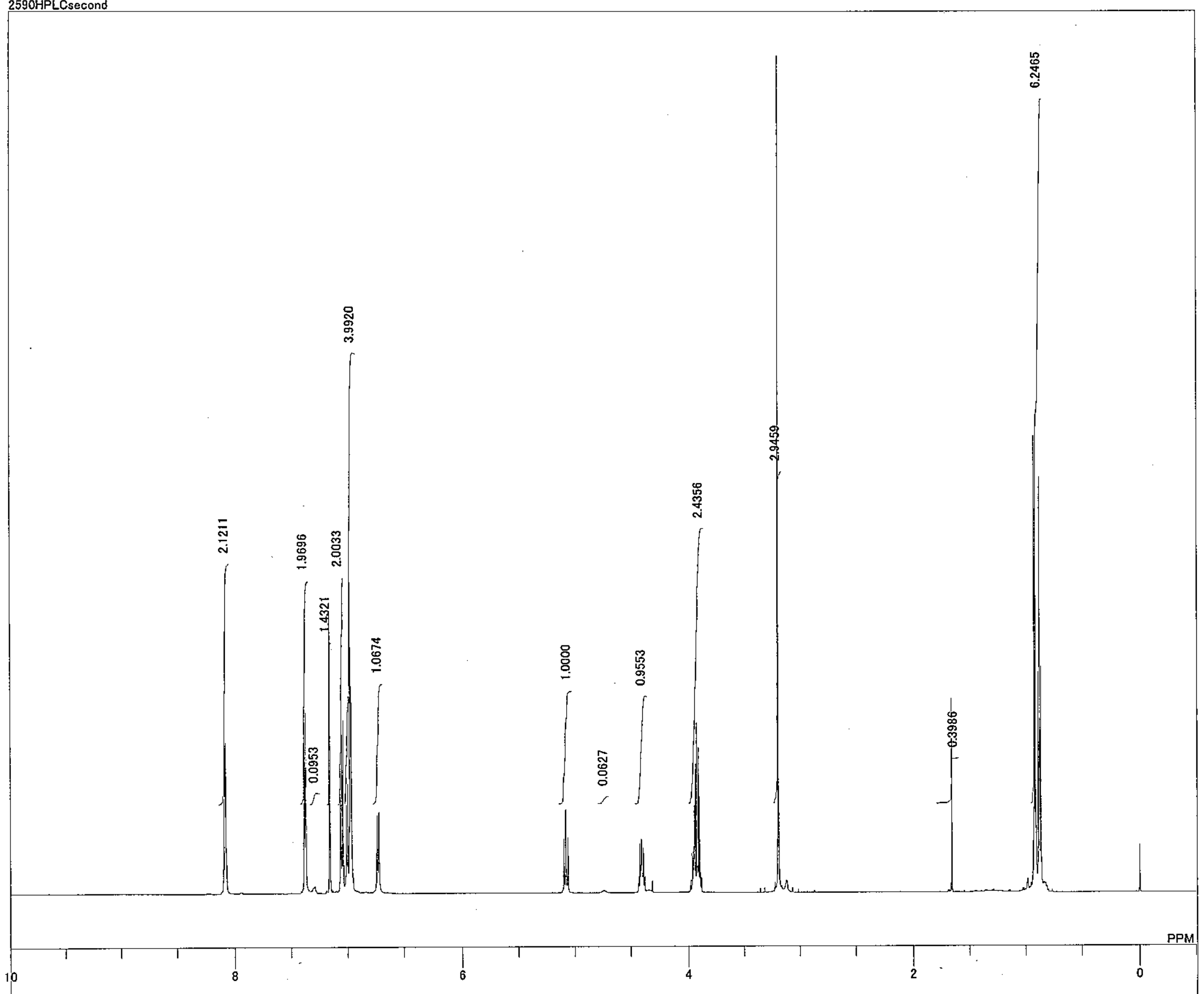

DFILE $\quad$ C:*Documents and Settings $¥ A l l$ Users $¥$ Docu DATIM 240HPLCsecond

DANUC 1402

OBFRQ

OBSET

OBFIN

FREQU

SCANS

ACQTM
PD
PW1
IRNUC

CTEMP

SLVNT

EXREF

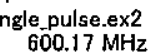
$5.30 \mathrm{KHz}$

$5.47 \mathrm{~Hz}$

$11261.26 \mathrm{~Hz}$

16
$1.4549 \mathrm{sec}$

$4.0000 \mathrm{sec}$

IH $21.7 \mathrm{c}$

${ }^{\mathrm{C} 6 \mathrm{D} 6}{ }_{0.00 \mathrm{ppm}}$

$0.00 \mathrm{ppm}$
$0.12 \mathrm{~Hz}$

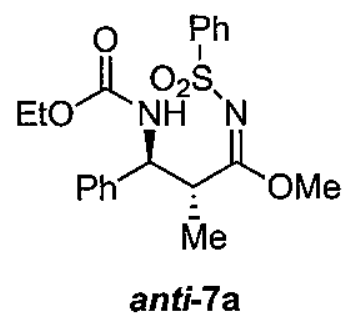


C: $¥$ Documents and Settings $¥$ All Users $¥$ Documents $¥$ Matsubara $¥ 2501-2600 ¥ 2590$ HPLCsecond_CARBON.als

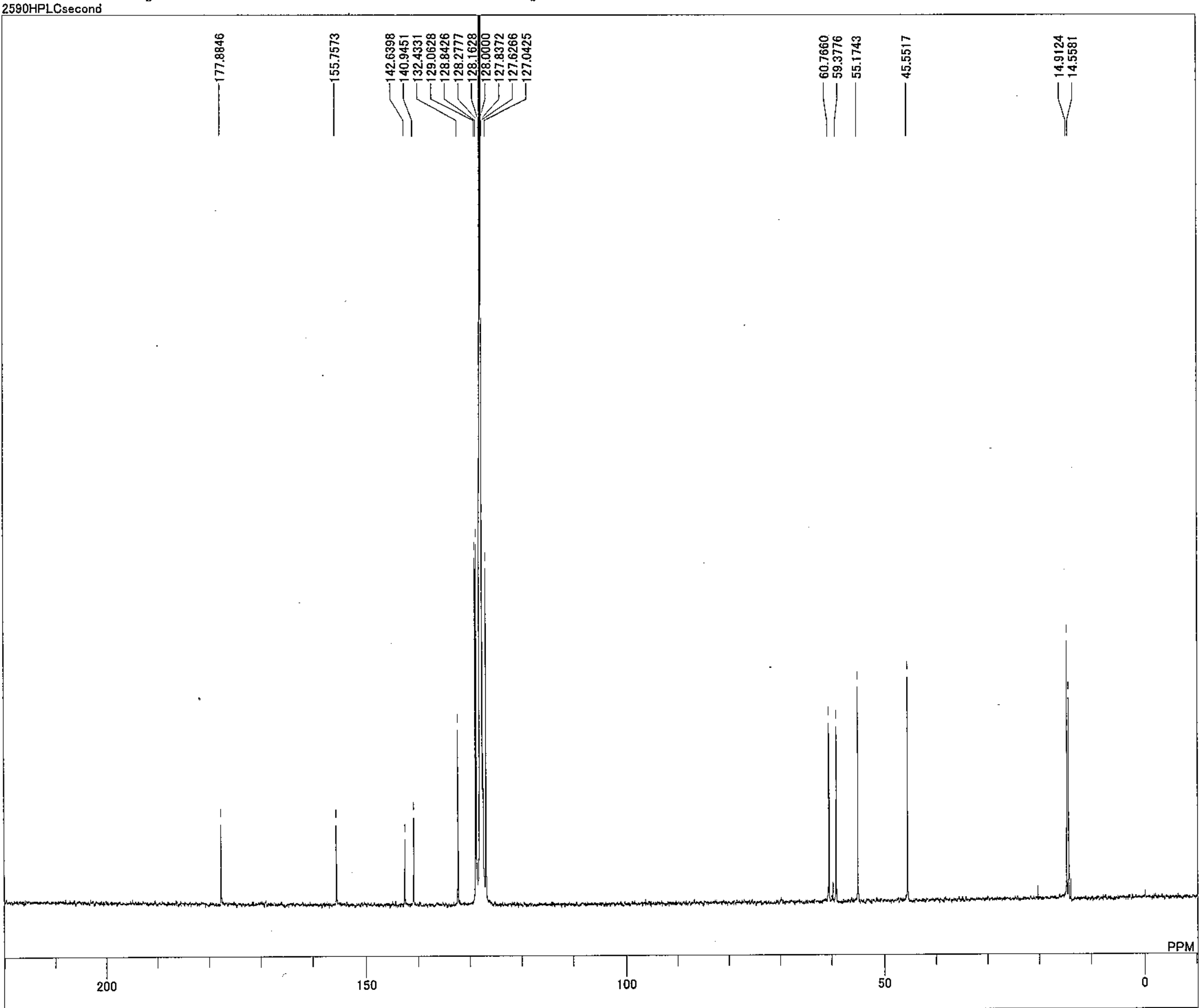

DFILE C:ҰDocuments and Settings¥All Users¥Docu

COATM 2J9HPLCsecond

OBNUC $13 \mathrm{C}$

OBFRQ

single puise_dec $150.92 \mathrm{MHz}$
$8.52 \mathrm{KHz}$
$1.74 \mathrm{~Hz}$

$\begin{array}{lc}\text { OBFIN } & 1.74 \mathrm{~Hz} \\ \text { POINT } & 32768\end{array}$

FCANS

ACQTM $\quad 0.6921 \mathrm{sec}$

$2.0000 \mathrm{sec}$

$1 \mathrm{H} \quad 3.45 \mathrm{usec}$

CTEMP $22.9 \mathrm{c}$

$\begin{array}{ll}\text { SLVNT } & \text { C6D6 } \\ \text { EXREF } & 128.00 \mathrm{ppm}\end{array}$

$\begin{array}{lc}\text { EXREF } & 128.00 \mathrm{ppm} \\ \text { BF } & 0.12 \mathrm{~Hz} \\ \text { RGAIN } & 56\end{array}$

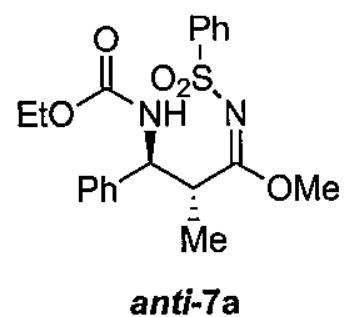

PPM 


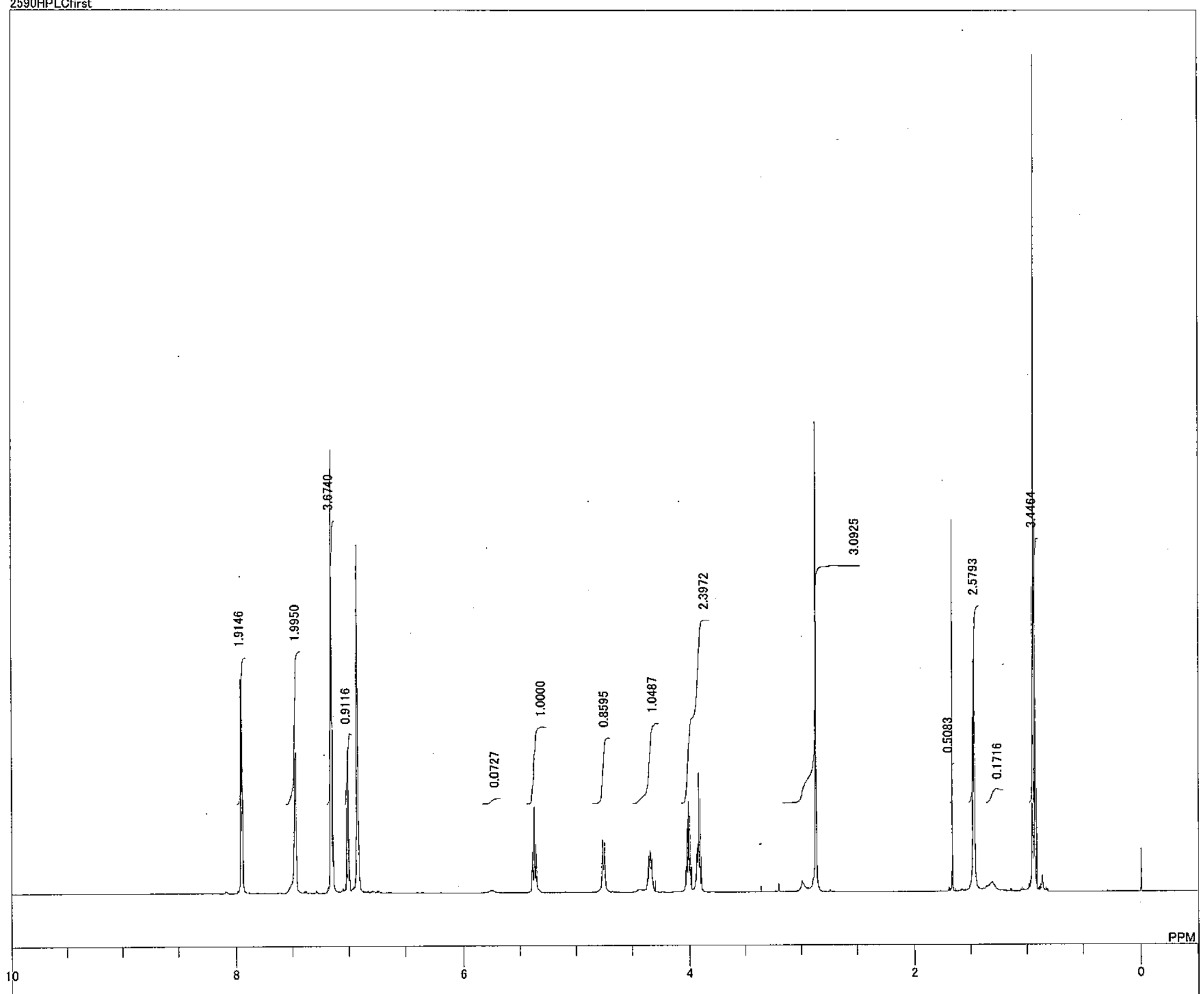

DFILE C:DDocuments and Settings:All Users¥Docu

COMNT

DATIM

OBSET

PBIN

SCANS

ACQTM

PWI

IRNUC

SLVNT

EXREF

BF

24-02-2007 02:45:04

single_pulse.ex2 $5.30 \mathrm{KHz}$ $1.47 \mathrm{~Hz}$

16384

16

$1.4549 \mathrm{sec}$

$4.0000 \mathrm{sec}$

$1 \mathrm{H} \quad 21.6 \mathrm{c}$

$0.00 \mathrm{ppm}$

$0.00 \mathrm{ppm}$
$0.12 \mathrm{~Hz}$
40

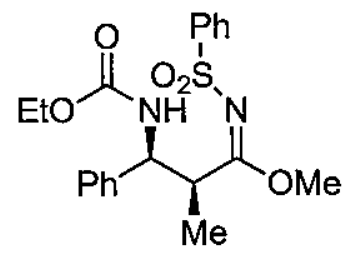

syn-7a 


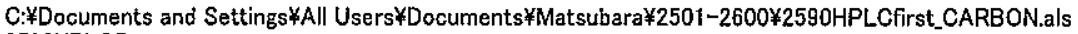

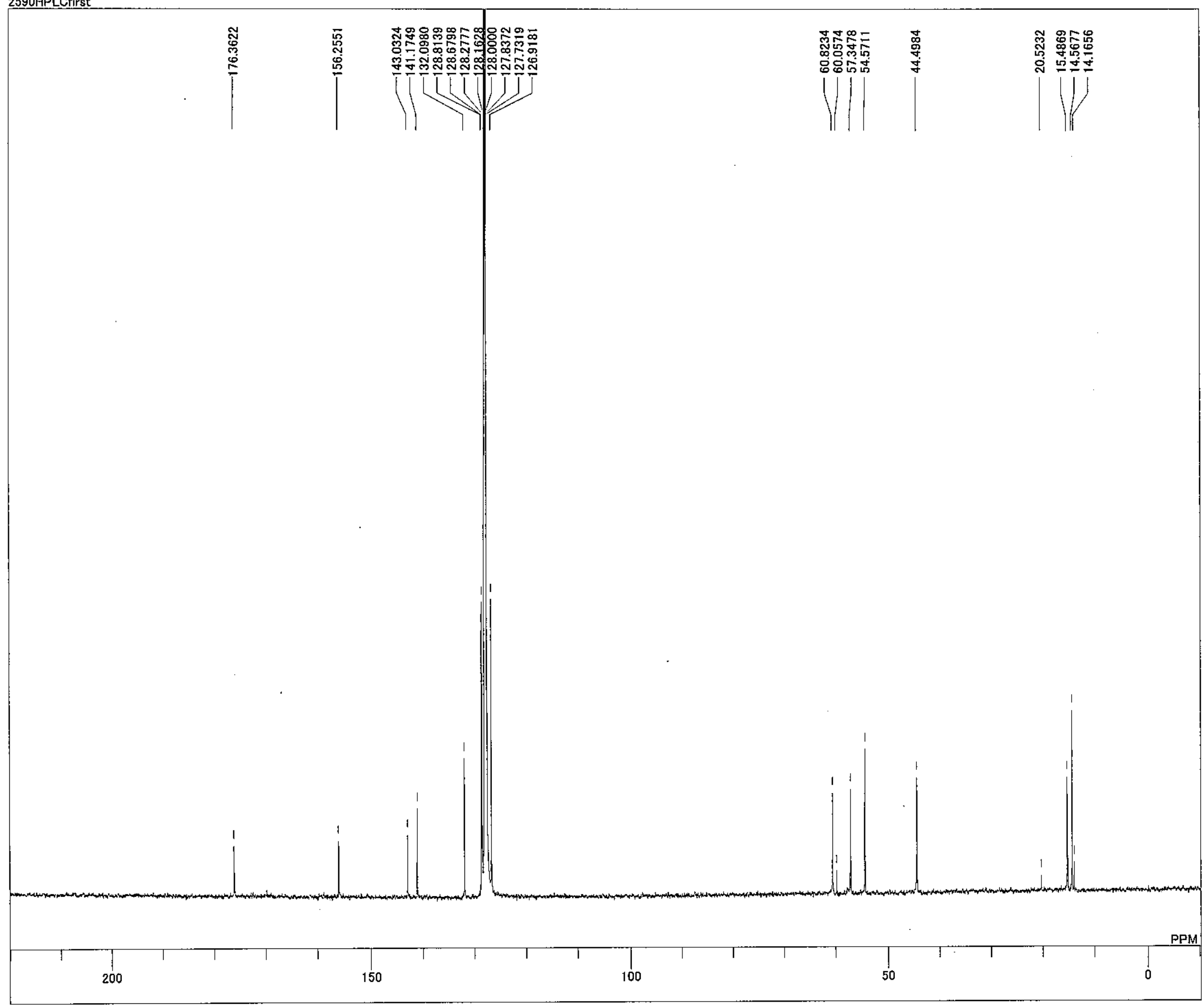

DFILE C: $:$ Docurnents and Settings $¥ A l l$ Users $¥$ Docu

COMNT

DENUC

EXMOD

OBFRQ
OBSET

OBFIN

POINT

PCANS

ACORM

PDI

IRNUC

CTEMP

SLVNT

(n)

4-02-2007 03:31:40

single-pulse_dec

$8.52 \mathrm{KHz}$ $1.74 \mathrm{~Hz}$

$47348.48 \mathrm{~Hz}$

024

$0.6921 \mathrm{sec}$

$2.0000 \mathrm{sec}$
3.45 usec

IH $23.0 \mathrm{c}$

$128.00 \mathrm{ppm}$

$0.12 \mathrm{~Hz}$

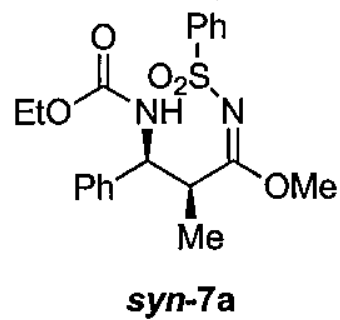


C:FDocuments and Settings $¥ A$ All Users*Documents $¥$ florian $¥ 600 \mathrm{MHz} Z$ fb 339 ptlc2major_PROTON.als fb339ptlc2major
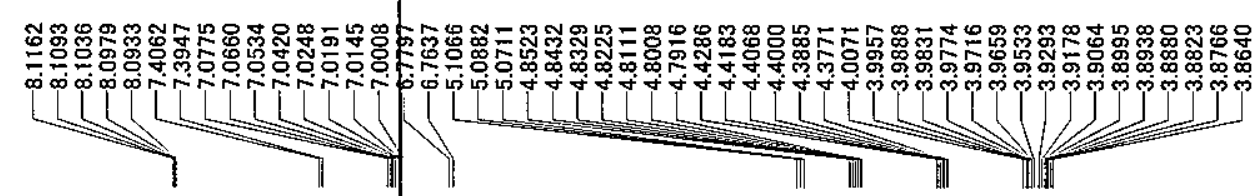

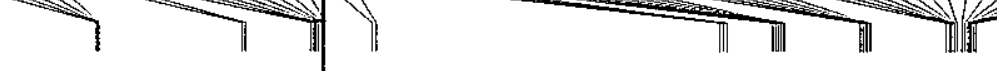

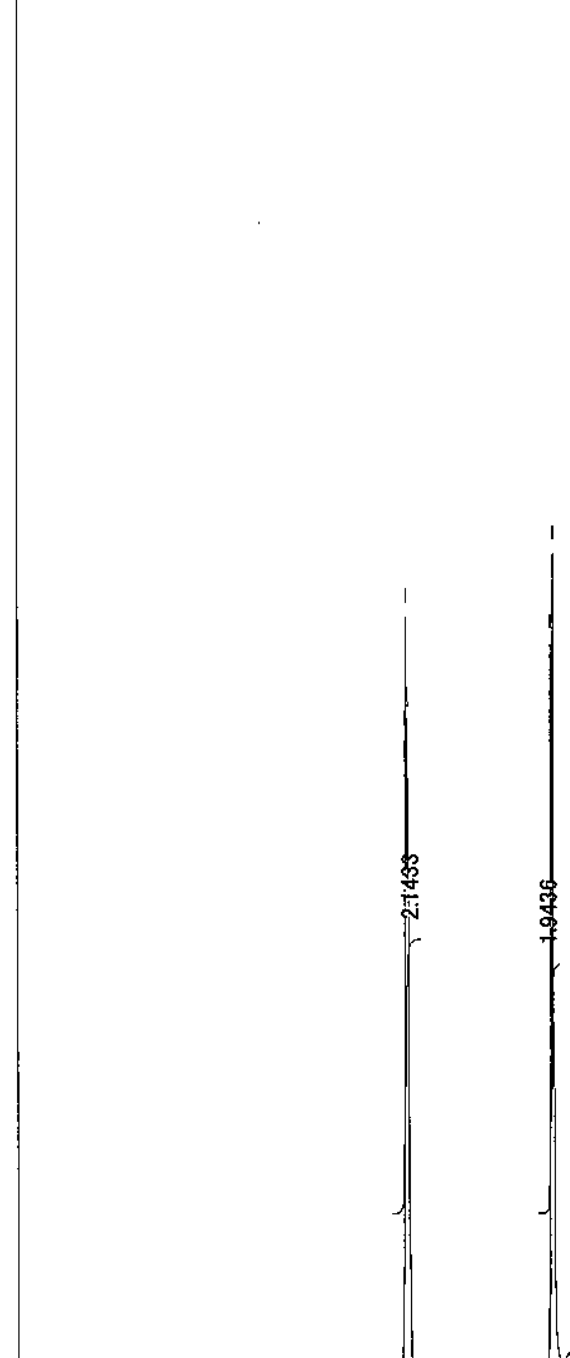

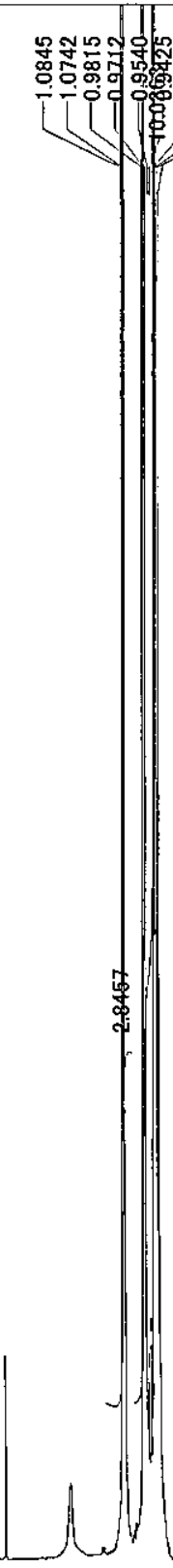

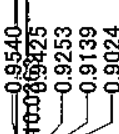

彳⿳亠口冋口十

DFLE

DATIM

EXMOD

OBFRQ

OBSET

PBNT

FREQU

SCANS

PD

PW1

TRNG

SLVMNT

EXREF

RGAIN

Documents and Settings:AIl Users $¥ D$ ocu 24-08-2007 19:34:52

ingle.pulse.ex2 $5.30 \mathrm{KHz}$ $5.47 \mathrm{~Hz}$ $1126126 \mathrm{~Hz}$

$11261.26 \mathrm{~Hz}$ 16
$1.4549 \mathrm{sec}$

$4.0000 \mathrm{sec}$

$1 \mathrm{H}$
$1.10 \mathrm{usec}$

C6D6 ${ }^{29.7}$

$0.00 \mathrm{ppm}$
$0.12 \mathrm{~Hz}$

${ }_{30}^{0.12 \mathrm{~Hz}}$

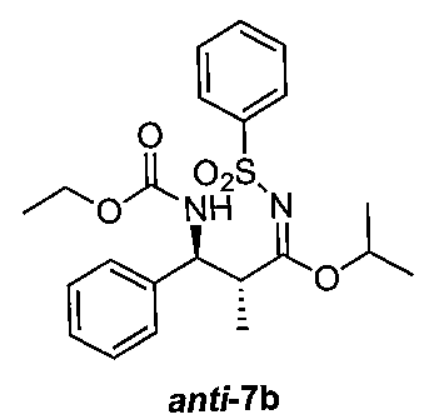




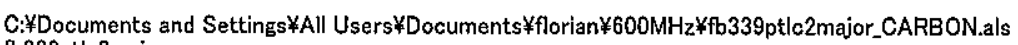

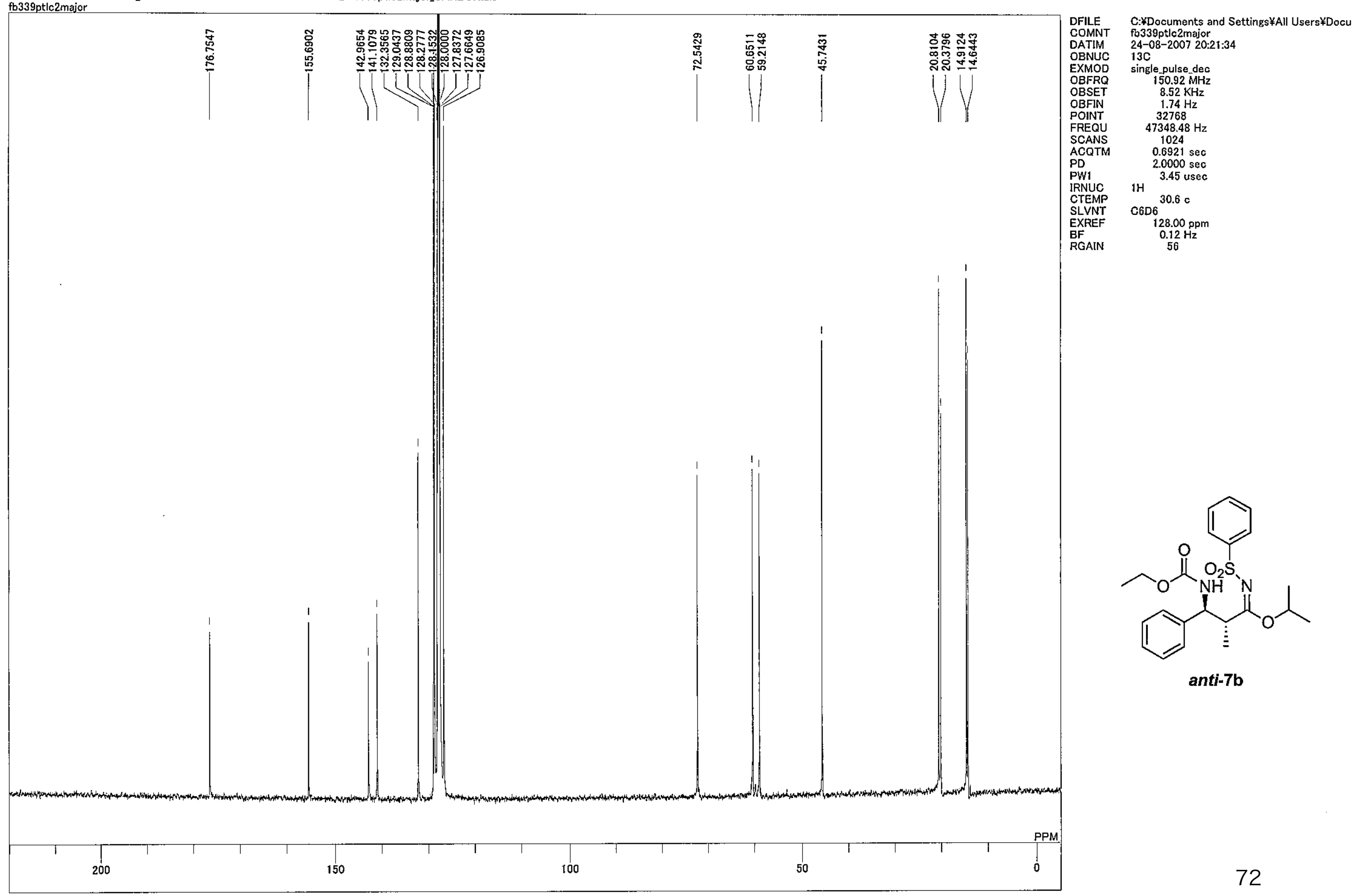



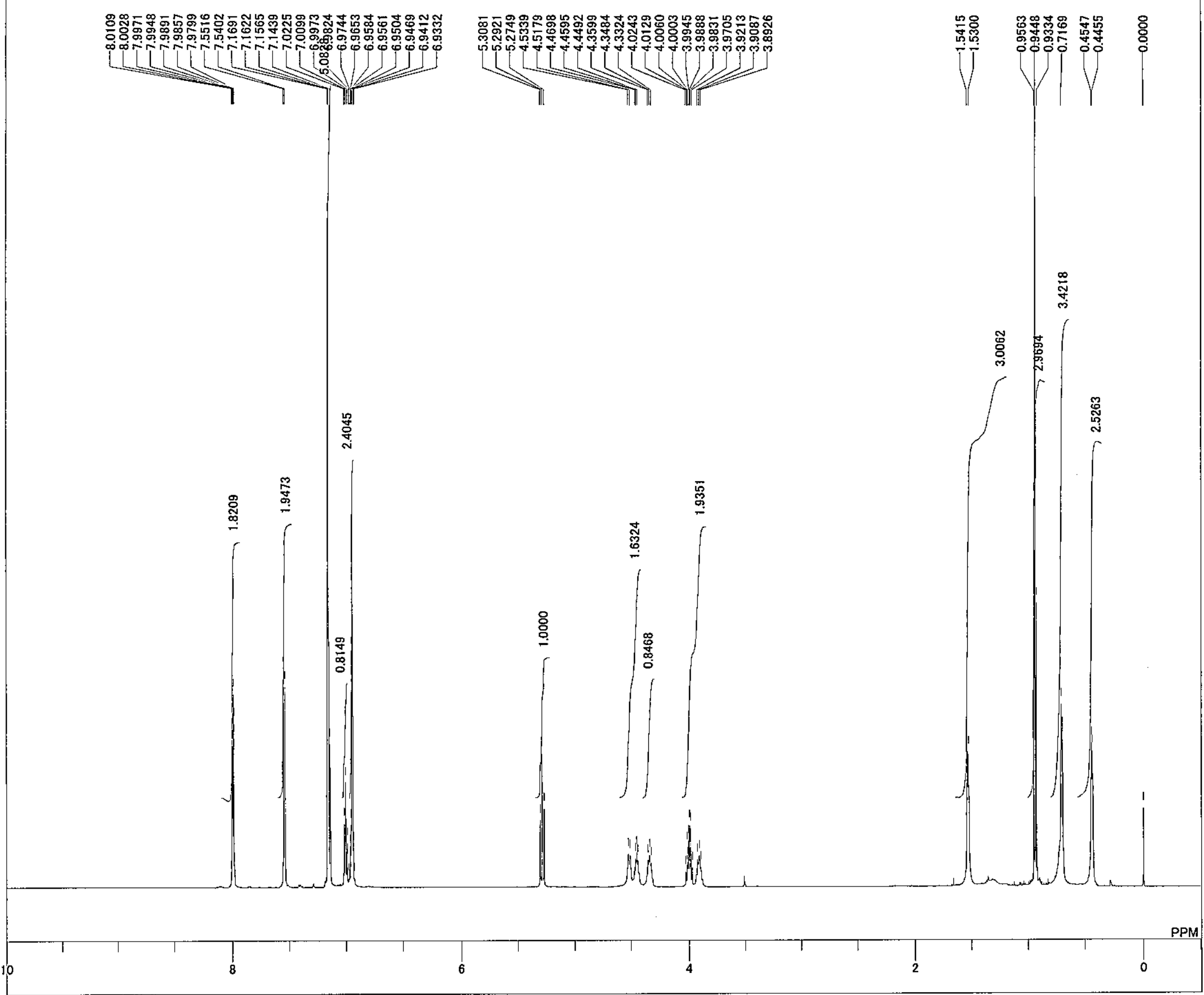

DFILE
COMNT

C:FDocuments and Settings:A.l Users*Doc 

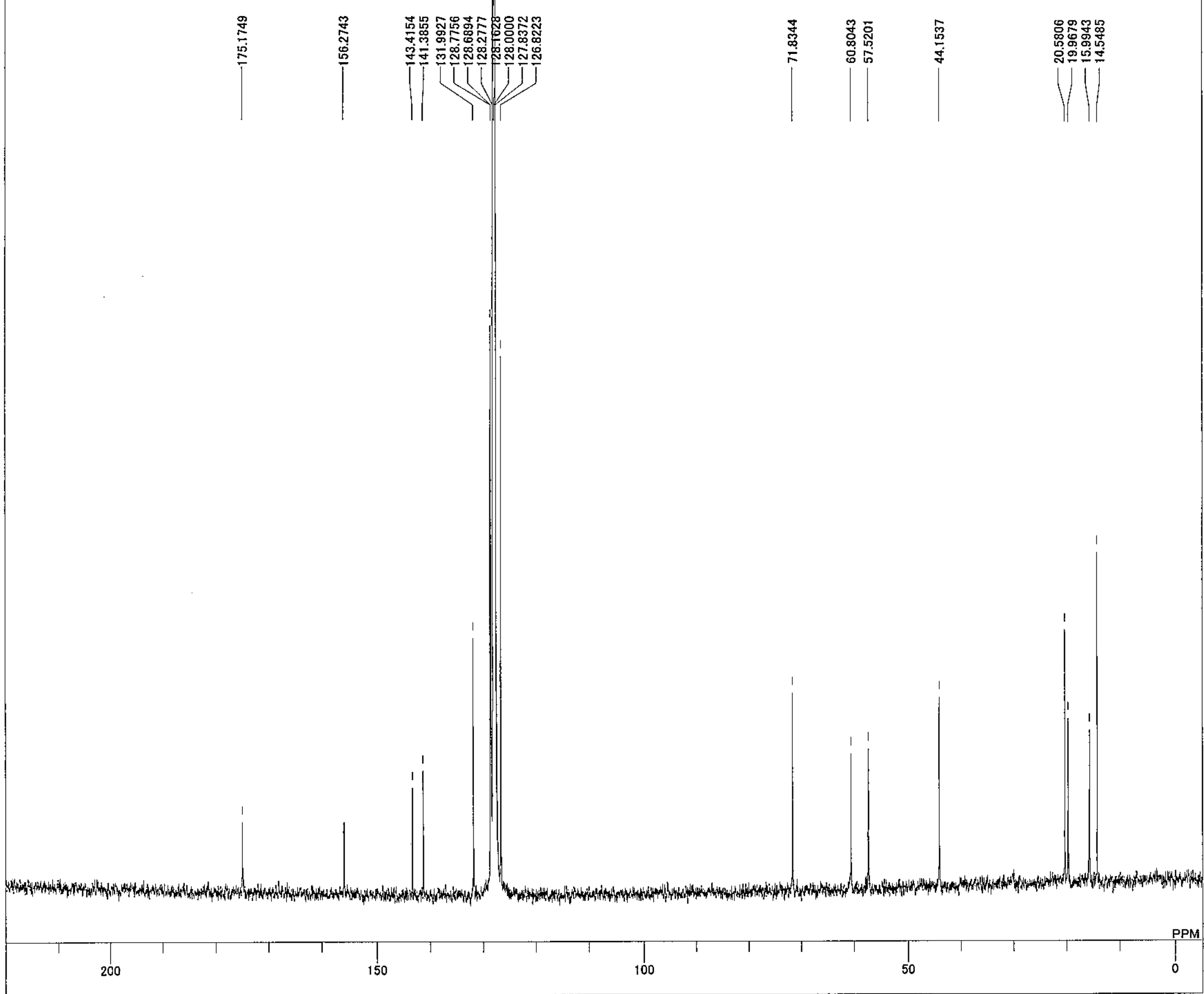

DFILE C:¥Documents and Settings¥All Users¥Docu

single_pulse_dec

i50.92 $\mathrm{MHz}$

$8.52 \mathrm{KHz}$

$1.74 \mathrm{~Hz}$

32768
$4734.48 \mathrm{~Hz}$

$0.6921 \mathrm{sec}$
$2.0000 \mathrm{sec}$

3.45 use

C6D6 $30.6 \mathrm{c}$

$128.00 \mathrm{ppm}$
$0.12 \mathrm{~Hz}$

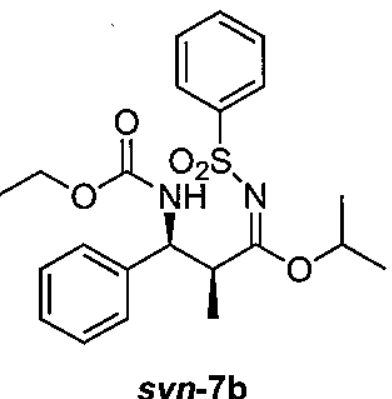

syn-7b 
C. Documents and Settings $¥ A$ All Users $¥ D$ Documents $¥$ Matsubara $¥ 2601-2700 * 2615$ after2ndpurification-1.als

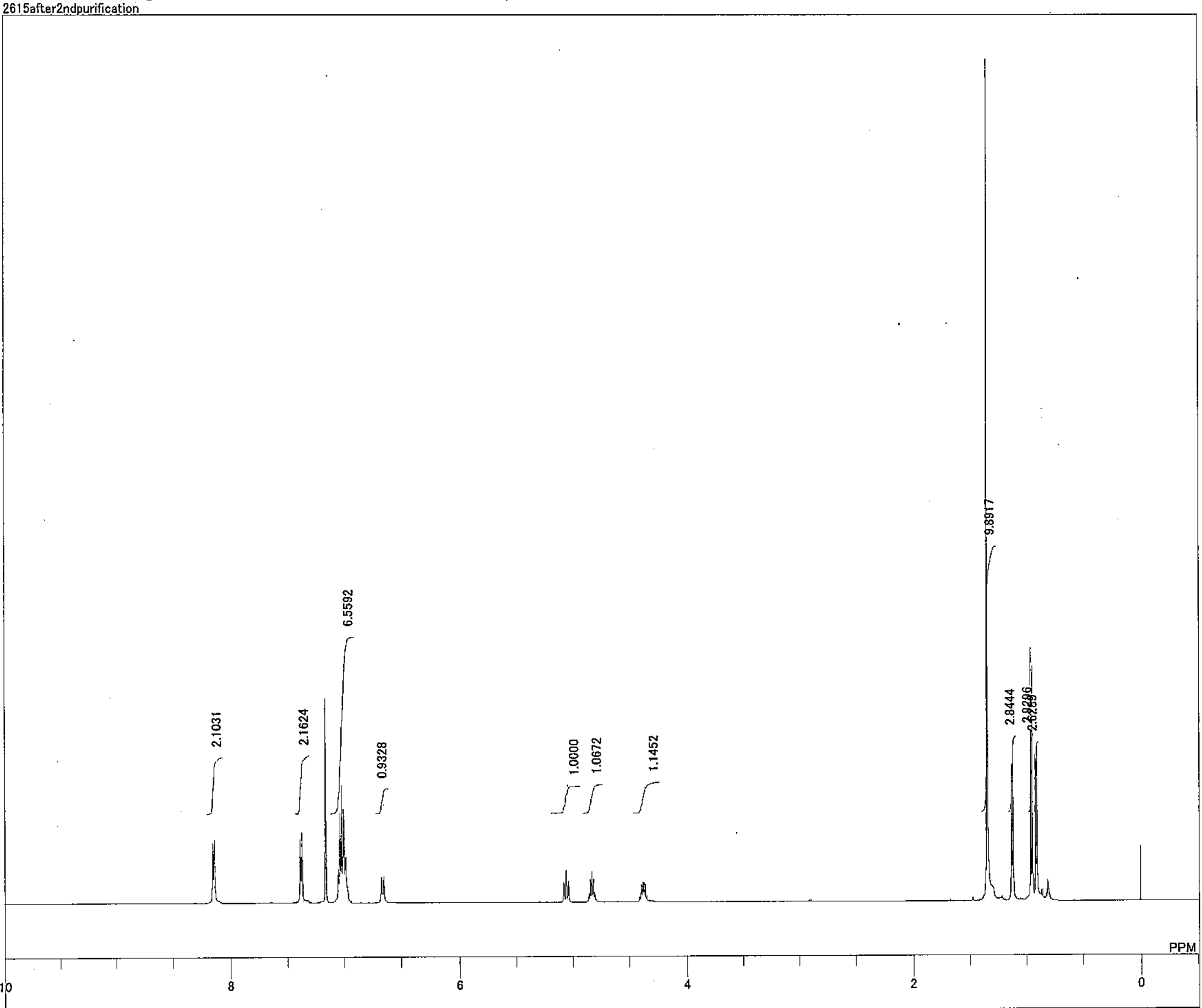

DFILE

COMNT

EXMOD

OBSET

OBFIN

POINT

FREQU

ACQTM

PD

PWI

CTEMP

SLVNT

EXXEF

RGAIN

Documents and Settings $¥ A l l$ Users*Do

2615after2ndpurification

iH NMR.ex2

$495.13 \mathrm{MHz}$
$4.38 \mathrm{KHz}$

$9.64 \mathrm{~Hz}$

13107

8

$1.7642 \mathrm{sec}$

$5.0000 \mathrm{sec}$

$1 \mathrm{H}$

$\mathrm{C} 6 \mathrm{D} 6{ }^{26.1}$

$0.00 \mathrm{ppm}$
$0.12 \mathrm{~Hz}$
34

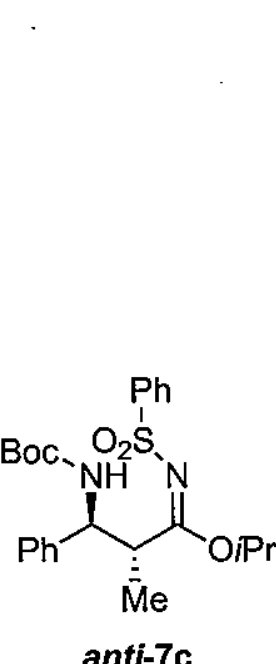

anti-7c 


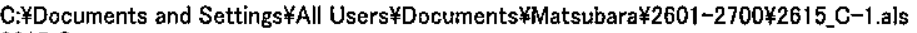

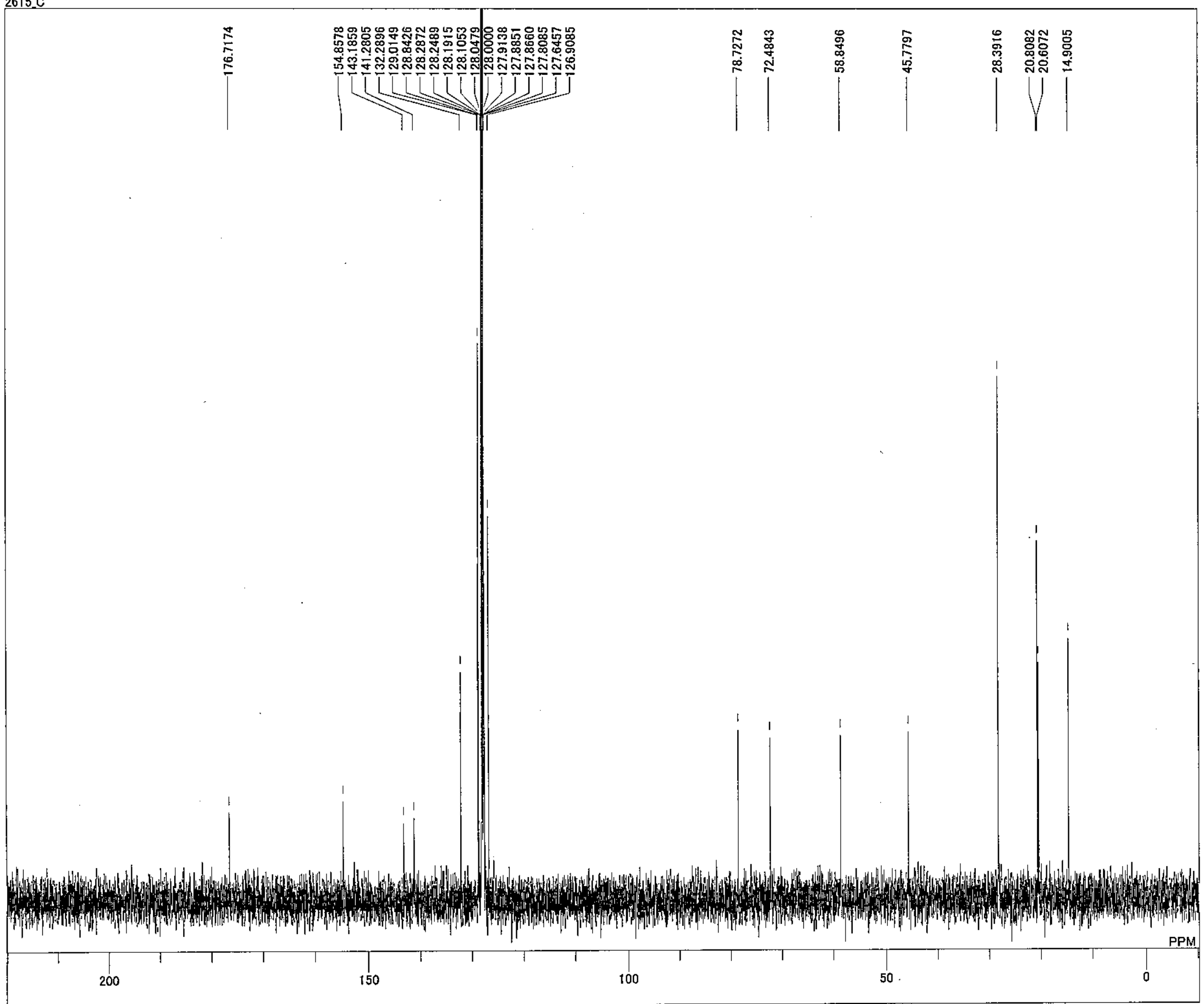

DFILE

C:FDocuments and SettingsFAll Users:Doqu

DATIM

OBSET

OBFIN

OBINT

SCANS

PD

IRNUC

CTEMP

EXREF

BF

RGAIN

7-2007 21:03.27

3C NMR.ex2

$3.45 \mathrm{KHz}$

$6.00 \mathrm{~Hz}$

26214

111

$0.8389 \mathrm{sec}$

$2.0000 \mathrm{sec}$
3.67 usec

1 H 26.5

C6D 6

$128.00 \mathrm{ppm}$
$0.12 \mathrm{~Hz}$

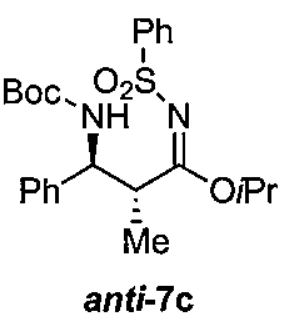




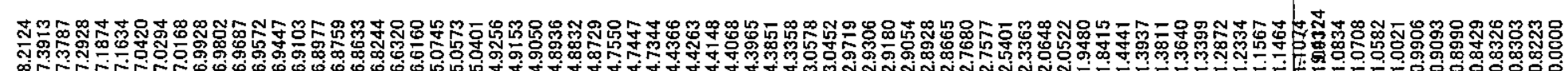

N.

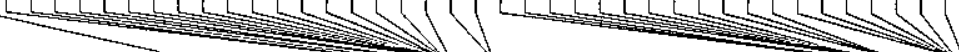

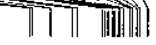

11

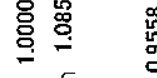

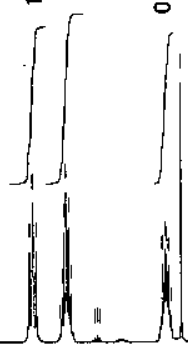

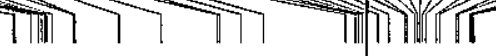

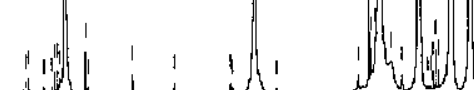

DFILE

DATIM

DATIM

OBFRQ

OBSET

OBIN

POINT

SCANS

ACQTM

${ }_{\mathrm{PW} 1}^{\mathrm{PD}}$

CTEMP

SLVNT

EXREF

RGAIN

GAIN
C:*Documents and Settings*ALICE2¥デスク Crubremade

single_pulse.ex2 $600.17 \mathrm{MHHz}$ $5.30 \mathrm{KHz}$ 20480 20480
$14076.79 \mathrm{~Hz}$ $1.4549 \mathrm{se}$
$4.0000 \mathrm{sec}$ $4.0000 \mathrm{sec}$

$1 \mathrm{H}$

C6D6 ${ }^{29.4 \mathrm{C}}$ $0.00 \mathrm{ppm}$
$0.12 \mathrm{~Hz}$

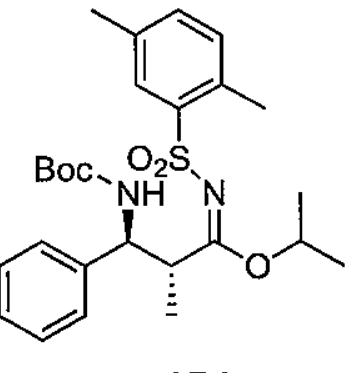

anti-7d 


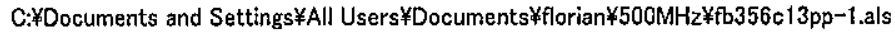

single pulse decoupled gated NOE

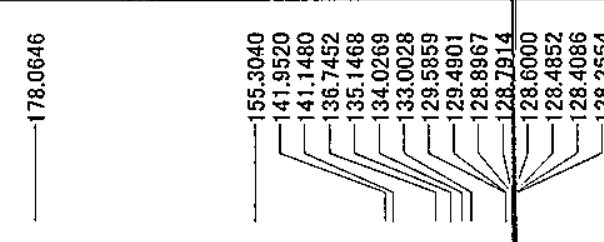

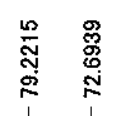

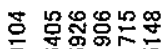 \\ A \\ (1)

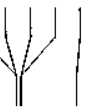

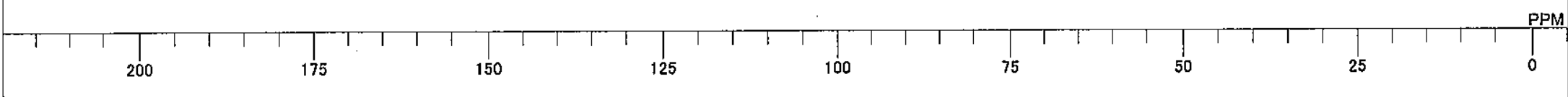

DFILE

COMNT

OBNUC

EXMOD

OBFRQ

OBFIN

SCANS

ACQTM

PD

IRNUC

CTEMP
SLVNT

BF
C:FDocuments and Settings $¥ A l l$ Users $¥$ Docu pulse decoupled gated NOE

3C NMR.ex2 $124.51 \mathrm{MHz}$ $3.45 \mathrm{KHz}$ $6.00 \mathrm{~Hz}$

$31249.52 \mathrm{~Hz}$

116 $2.0000 \mathrm{sec}$

$1 \mathrm{H} .67 \mathrm{~s}$

$25.5 \mathrm{c}$

$128.60 \mathrm{ppm}$ $1.20 \mathrm{~Hz}$

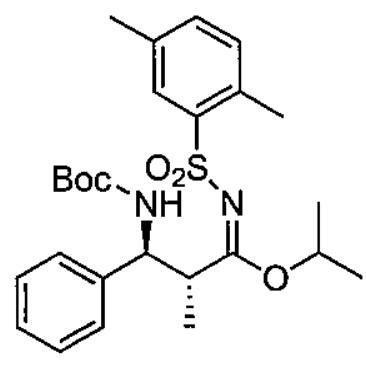

anti-7d 


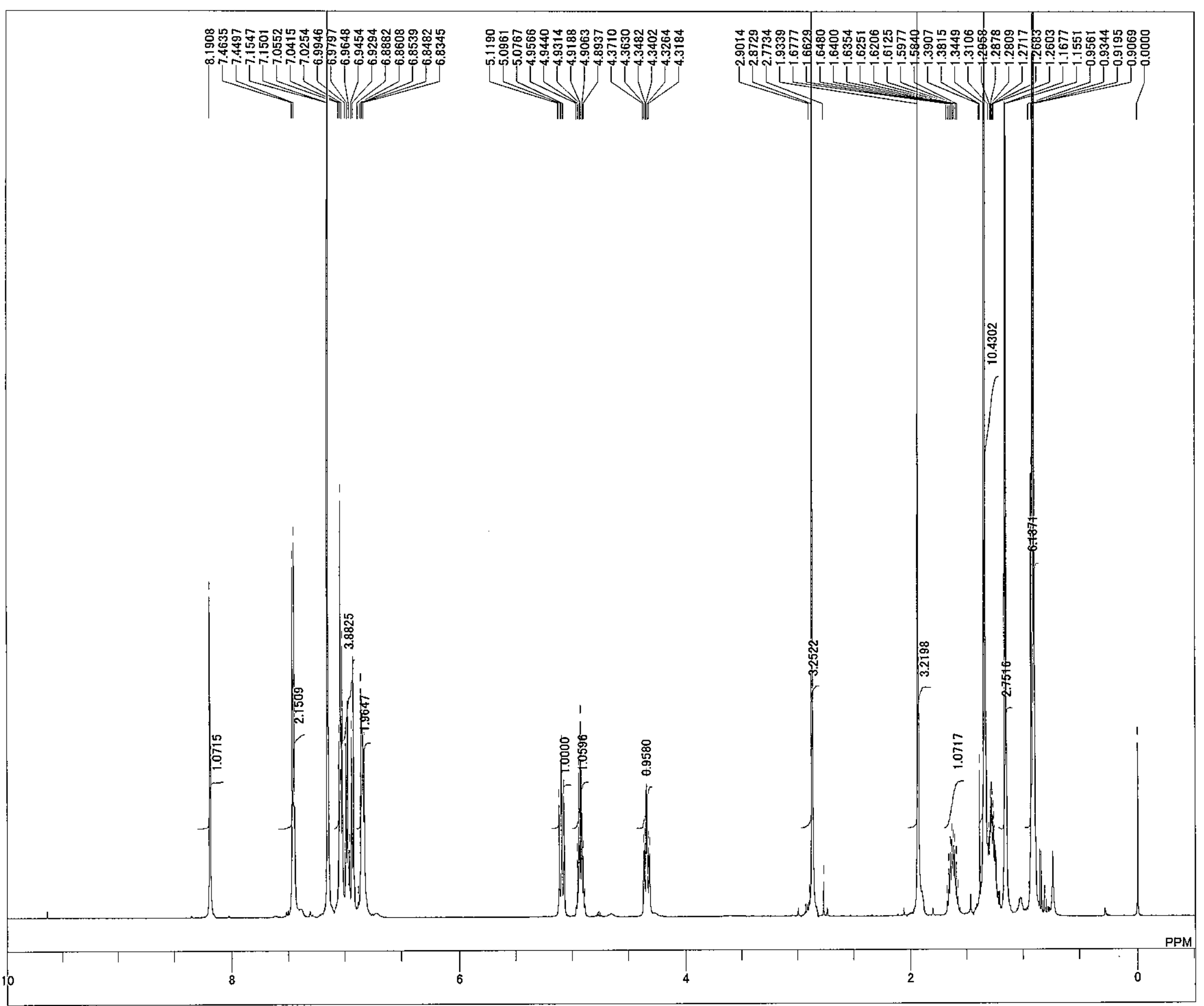

DFILE

DATIM

DATIM

OBSET

OBFIN

POINT

SCANS

ACQTM

PW1

IRNUC

SLVNT

EXREF

RGAIN

Eca*data*florian¥fb406-1.jdf

0-08-2007 21:09:25

H NMR.ex2

$495.13 \mathrm{MHz}$
$4.38 \mathrm{KHz}$

$9.64 \mathrm{~Hz}$

16400

$1.7642 \mathrm{sec}$

$5.0000 \mathrm{sec}$

$1 \mathrm{H}$

C6D6 27.6

$0.00 \mathrm{ppm}$
$0.12 \mathrm{~Hz}$

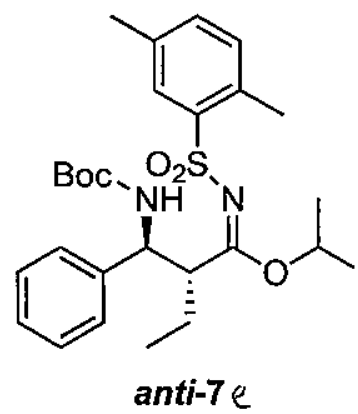




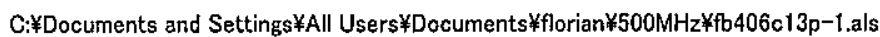

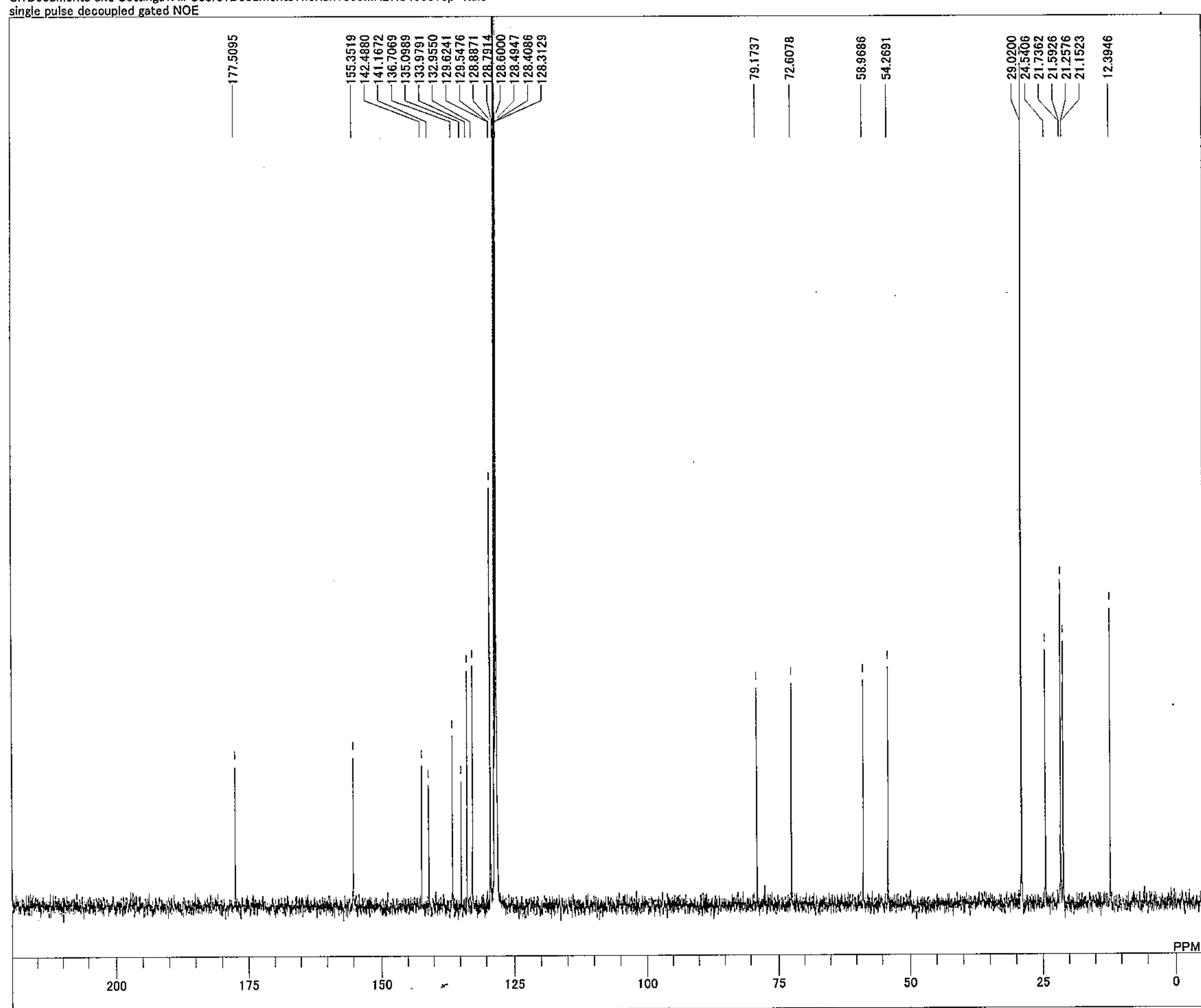

DFILE C:FDocuments and Settings:All Users*Docu

single pulse decoupled gated NOE

DATIM

EXMOD

OBFRQ

OBSET

PBFIN

FREQU

SCANS

$\mathrm{PD}$

PWNUC

CTEMP

SLVNT

RGAIN

13C NMR.ex2

$124.51 \mathrm{MHz}$
$3.45 \mathrm{KHz}$ $6.00 \mathrm{~Hz}$

$31249.52 \mathrm{~Hz}$

62
$0.8389 \mathrm{sec}$
$2.0000 \mathrm{sec}$

$2.0000 \mathrm{sec}$
3.67 usec

C6D6 $25.7 \mathrm{c}$

$128.60 \mathrm{ppm}$

$1.20 \mathrm{~Hz}$
52

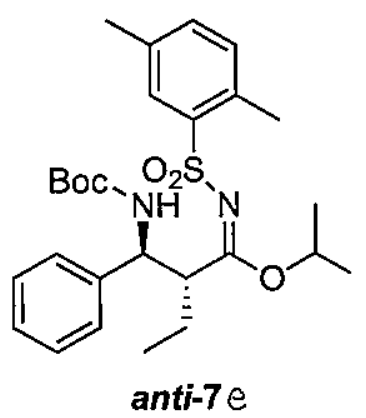


DFILE

COMN

DATIM

EXMOD

OBFRQ

OBSET

OBFIN

FREQU

FREQU
SCANS

ACQTM

PD

IRNUC

CTEMP

SLVNT

BFAIN

Documents and Settings*All Users*Docu 19:22:04

IH NMR.ex2

$495.13 \mathrm{MHz}$
$4.38 \mathrm{KHz}$

$9.64 \mathrm{~Hz}$

$7429.31 \mathrm{~Hz}$

$1.7642 \mathrm{sec}$

$5.0000 \mathrm{sec}$
$6.50 \mathrm{usec}$

$1 \mathrm{H} \quad 24.5 \mathrm{c}$

C6D6 0.5

$0.00 \mathrm{ppm}$
$0.12 \mathrm{~Hz}$
28

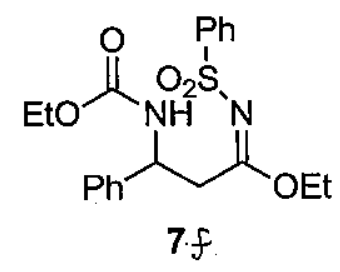

PPM 


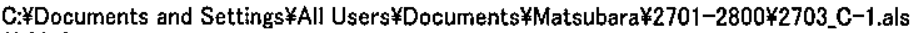

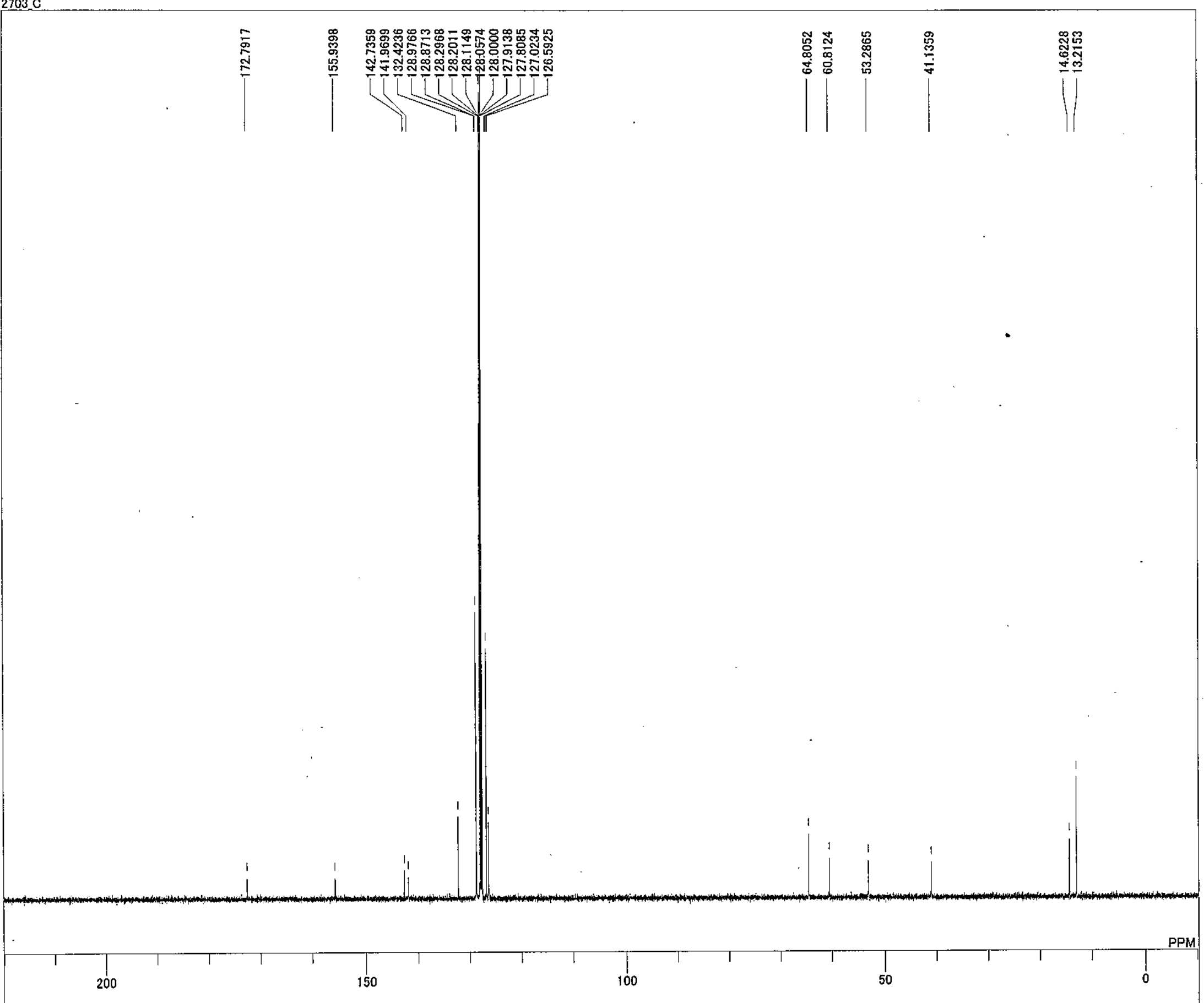

OFILE C:FDoclsments and Settings $¥ A$ All Users $¥$ Docu

DATIM $10307-20071927: 44$

XMOD

13C NMR.ex 2

$\begin{array}{ll} & 124.51 \mathrm{MHz} \\ \text { OBSET } & 3.45 \mathrm{KHz}\end{array}$

$\begin{array}{lll}O B & \\ O B F I N & 600 \mathrm{~Hz}\end{array}$

FREQU $\quad 3124952 \mathrm{~Hz}$

SCANS $\quad 106$

ACQTM $\quad 0.8389 \mathrm{sec}$

$2.0000 \mathrm{sec}$

IH 3.67 usec

CTEMP $24.9 \mathrm{c}$

SLVNT C6D6

$\begin{array}{ll}\text { EXREF } & 128.00 \mathrm{ppm} \\ \mathrm{BF} & 0.12 \mathrm{~Hz}\end{array}$

RGAIN

$0.12 \mathrm{~Hz}$

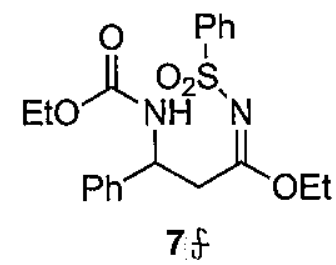

PM 


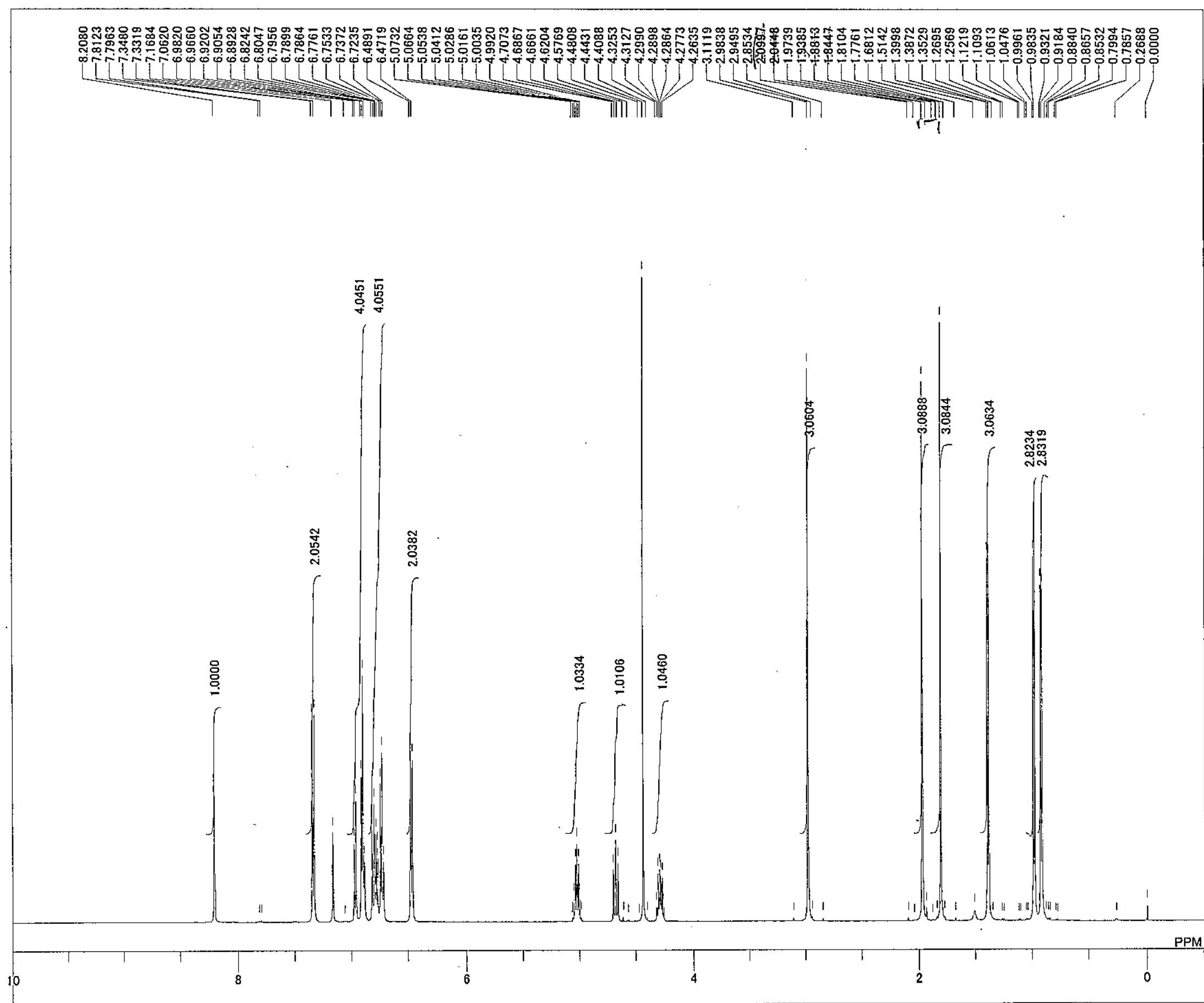

DFILE

DATIM

OBNUC

OBFRQ

OBSET

OBFIN

FREQU

ACQTM

PW1

RNUC

CTEMP

SLVNT

BF

BGAIN

Eca $a$ data $¥$ florian¥fb434last $-3 . j d f$

24-08-2007 08:29:32

IH NMR.ex2

$495.13 \mathrm{MHz}$

$4.38 \mathrm{KHiz}$

$9.64 \mathrm{~Hz}$

$9286.78 \mathrm{~Hz}$

8
$\cdot 1.7642 \mathrm{sec}$

$5.0000 \mathrm{sec}$

$27.9 \mathrm{c}$

$0.00 \mathrm{ppm}$

$0.00 \mathrm{ppm}$
$0.12 \mathrm{~Hz}$
26

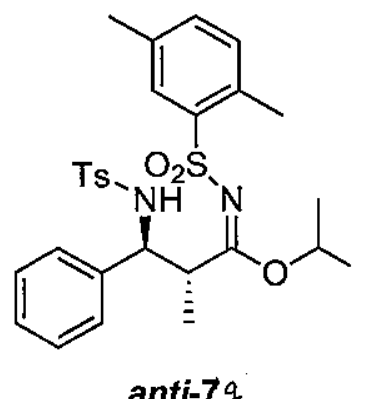

anti-7g 


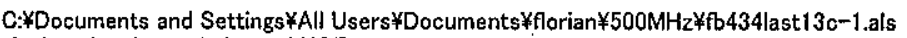

single pulse decoupled gated NOE
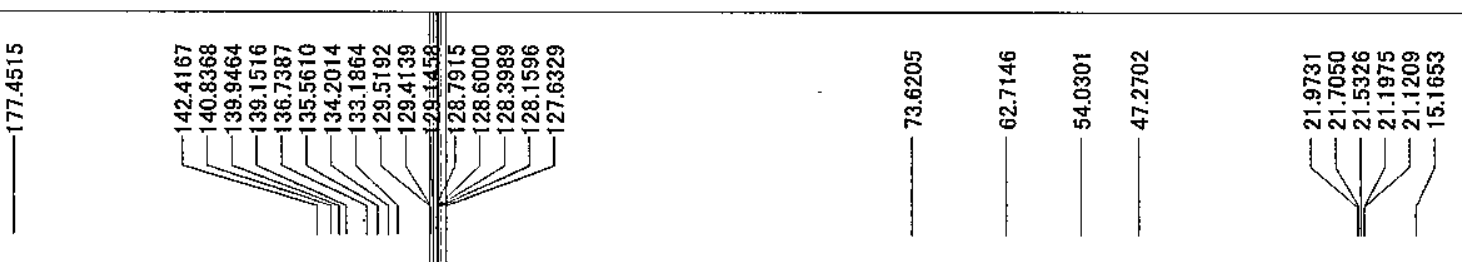

DFILE
COMNT

DABNUC

EXMOD

OBFRQ

OBSET

POINT

POINT
FREQU

SCANS

ACQTM

PD 1

PW1

CTEMP

SLVNT

EXREF

RGAIN

\#Documents and Settings:All Users $\because$ Docu

single pulse decoupled gated NOE

13C NMR.ex2

$124.51 \mathrm{MHz}$
$3.45 \mathrm{KHz}$

$6.00 \mathrm{~Hz}$

$31249.52 \mathrm{~Hz}$

442
$0.8389 \mathrm{sec}$
$2.0000 \mathrm{sec}$

$1 \mathrm{H} \quad 3.67$ usec

$6006^{28.5 \mathrm{c}}$

$128.60 \mathrm{ppm}$
$0.12 \mathrm{~Hz}$
52

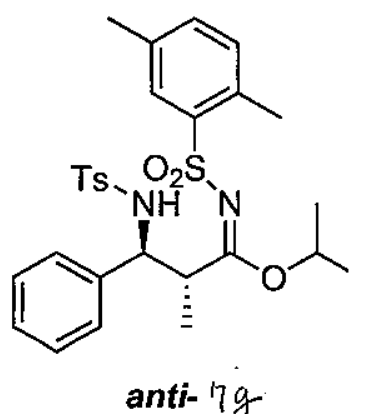




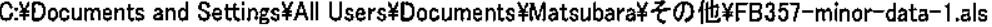

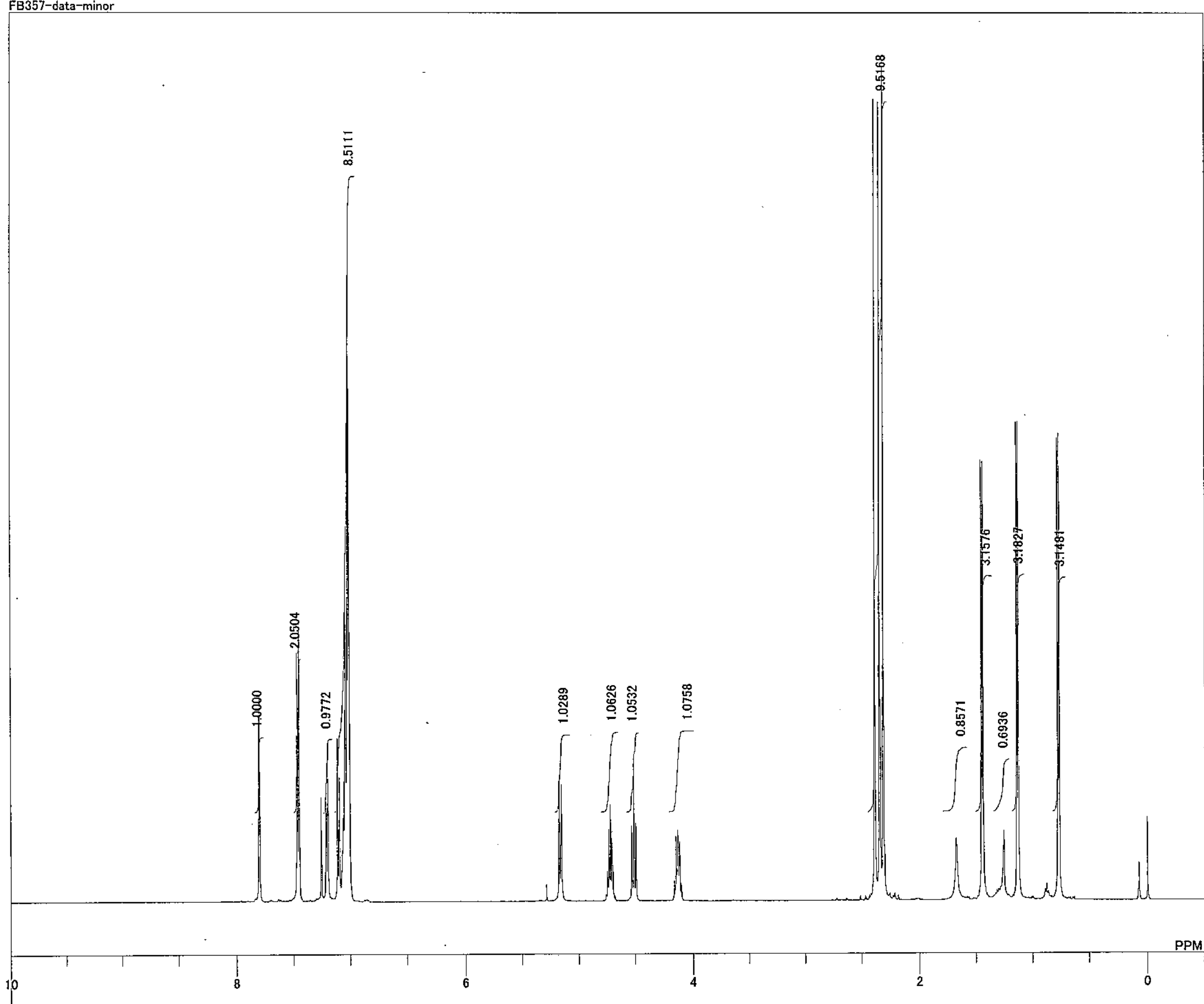

DFILE C:FDocuments and Settings $¥$ All Users:Docu

DOMNT TB357-data-minor

OBNUC IH

EXMOD 1H NMR.ex2

$\begin{array}{lc}\text { OBFRQ } & 495.13 \mathrm{MHz} \\ \text { OBSET } & 4.38 \mathrm{KHz}\end{array}$

$\begin{array}{ll}\text { OBSET } & 4.38 \mathrm{KHz} \\ \text { OBFIN } & 9.64 \mathrm{~Hz}\end{array}$

POINT 13107

FREQU $\quad 742931 \mathrm{~Hz}$

$\begin{array}{ll}\text { SCANS } & 8 \\ \text { ACOTM } & 1.7642 \mathrm{seC}\end{array}$

PD $\quad 5.0000 \mathrm{sec}$

IH 6.50 usec

CTEMP
SLVNT

SLVNT

EXREF

$0.00 \mathrm{ppm}$
$0.12 \mathrm{~Hz}$
38

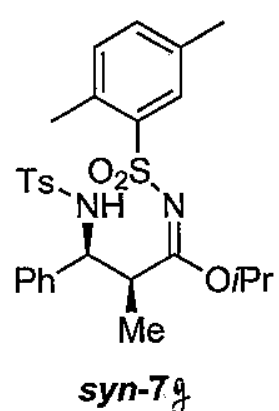


DFILE

DFILE
DATIM
OBNUC

OBNUC

EXMOD

OBFRQ

OBFIN

POIN

SCANS

ACQTM

PD 1

CTEMP

SLVNT

RGAIN

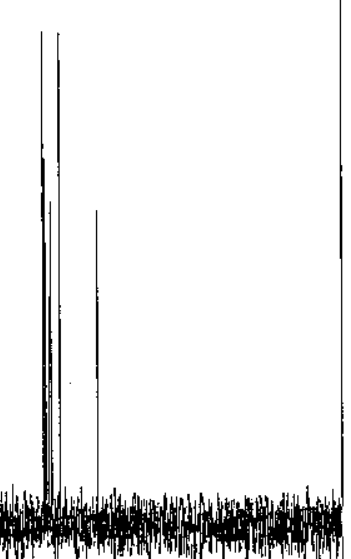

6. PPM
C:FDocuments and Settings:All Users*Docu $11-08-2007$ 13:47:55

$13 \mathrm{C}$ NMR.ex2 $124.51 \mathrm{MHz}$
$3.45 \mathrm{KHz}$ $6.00 \mathrm{~Hz}$ $31249.52 \mathrm{~Hz}$ $0.8389 \mathrm{se}$ $2.0000 \mathrm{sec}$ $29.9 \mathrm{c}$ $\mathrm{CDCL}^{29.9 \mathrm{c}}$ $77.00 \mathrm{ppm}$
$0.12 \mathrm{~Hz}$
54

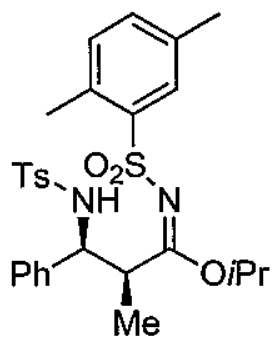
syn-7 $\mathrm{g}$. 
C::Documents and Settings¥ALICE2¥デスタトップ¥fb393remade_PROTON.3

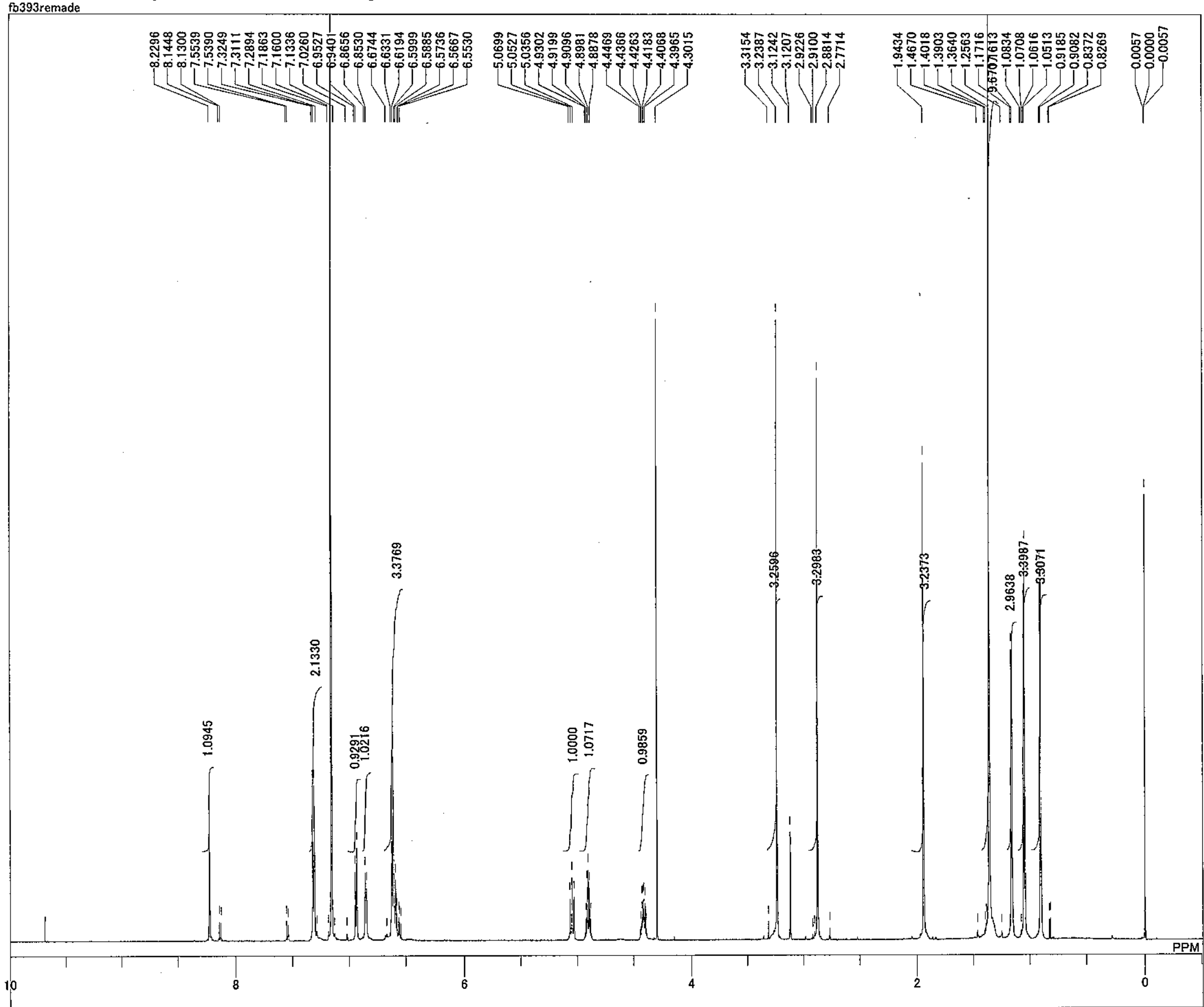

DFILE CFDocuments and Settings¥ALCE2¥デスク

28-08-2007 21:19:46

XMOD

OBFRQ $\quad 600.17 \mathrm{MHz}$

$\quad 5.30 \mathrm{KHz}$

$\quad 5.47 \mathrm{~Hz}$

FREQU $\quad 14076.79 \mathrm{~Hz}$

$\begin{array}{lc}\text { SCANS } & 16 \\ \text { ACQTM } & 1.4549 \mathrm{sec} \\ \text { PD } & 4.0000 \mathrm{sec}\end{array}$

$\begin{array}{lr}4.000 \mathrm{sec} \\ \text { PW1 } & 9.10 \mathrm{usec}\end{array}$

RNUC $1 \mathrm{H} \quad 29.4$

SLVNT C6D6

EXREF

RGAIN

$0.00 \mathrm{ppm}$
$0.12 \mathrm{~Hz}$
52

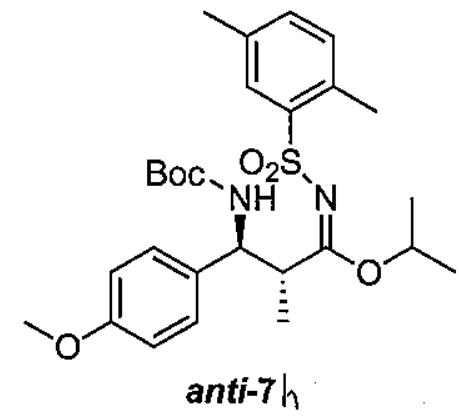

anti-7h 
C: $¥$ Documents and Settings $¥ A$ All Users $¥ D$ Documents $¥$ florian $¥ 500 \mathrm{MHZ} Z ¥ \mathrm{fb} 393 \mathrm{c} 13 \mathrm{pp}-1$.als single pulse decoupled gated NOE
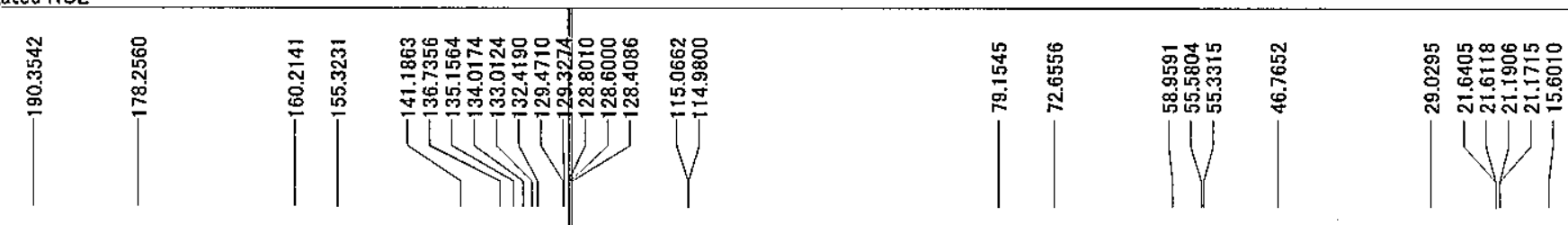

DFILE C:*Documents and Settings:All Users¥Docu

COMNT single pulse decoupled gated NOE

COMNIT

XMOD

OBFRQ

OBSET

POINT

FREQU

SCANS

PD

PW1

CTEMP
SLVNT

SLVNT

EXFEF

RGAIN

${ }_{13 \mathrm{C}}$ NMR.ex2

$124.51 \mathrm{MHz}$
$3.45 \mathrm{KHz}$

$6.00 \mathrm{~Hz}$

$31249.52 \mathrm{~Hz}$

62

$0.8389 \mathrm{sec}$

$2.0000 \mathrm{sec}$
$3.67 \mathrm{usec}$

1H 25.5 。

C6D6 $128.60 \mathrm{ppm}$

$28.60 \mathrm{ppm}$
$1.20 \mathrm{~Hz}$

(n)

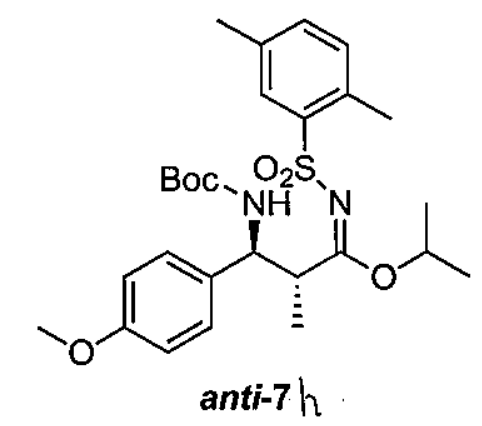

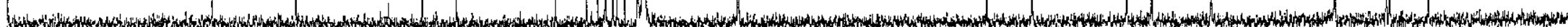
PPM 
C.\#Documents and Settings¥ALICE2¥デスクトップ¥fb378remade_PROTON.3

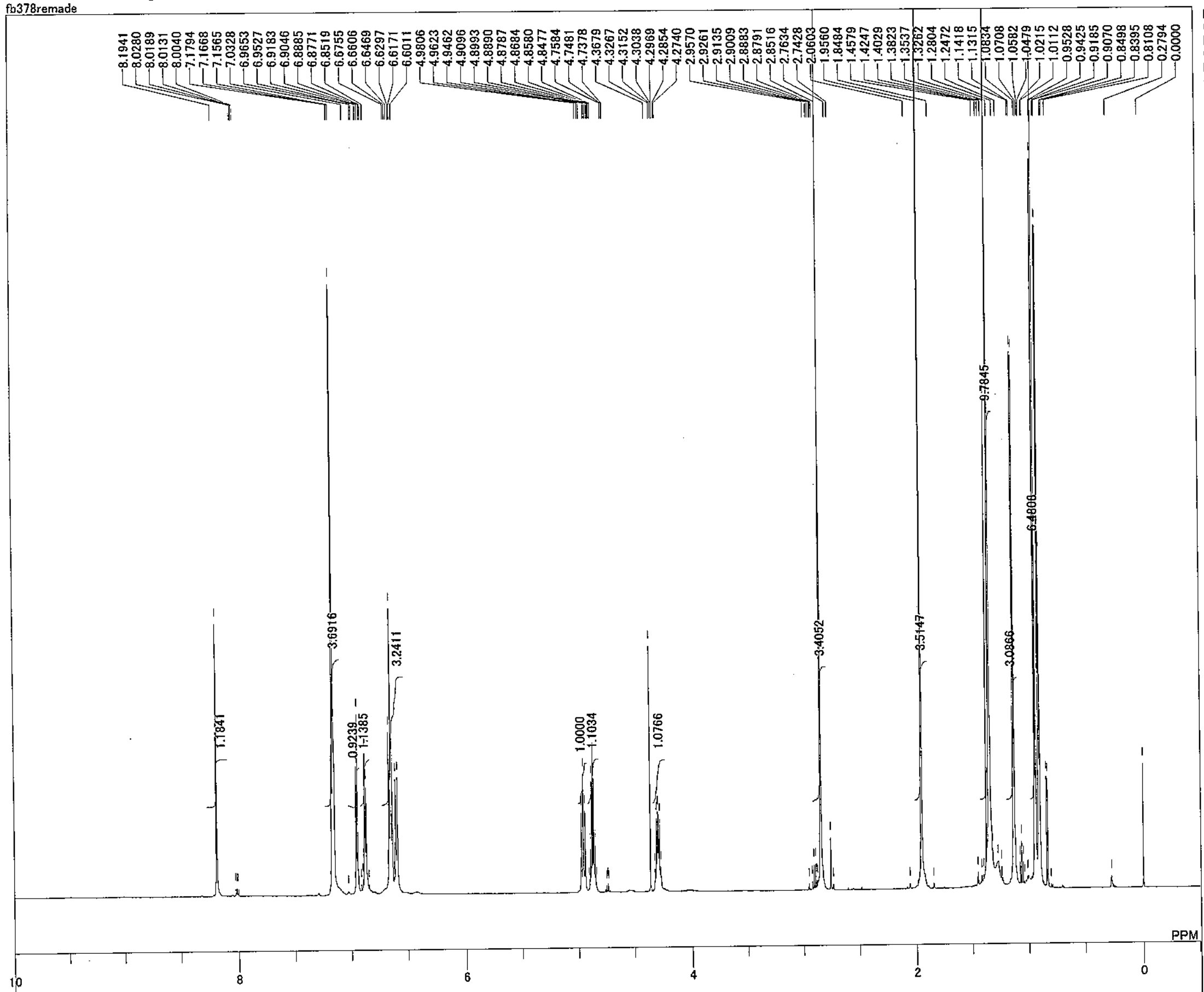

DFILE

XMOD

OBFRQ

OBSET

FREQU

ACQTM

PDi

PWNUC

SLEMP

RGAIN

${ }_{12.57}^{C 606}$

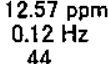

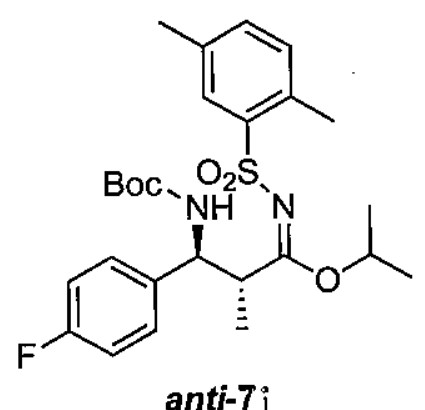


C:劷ocuments and Settings $¥$ Ail Users $¥ D$ ocuments $¥$ florian $¥ 500 \mathrm{MHz} \approx \mathrm{fb} 378 \mathrm{c} 13 \mathrm{pp}-1$. als single puise decoupled gated NOE
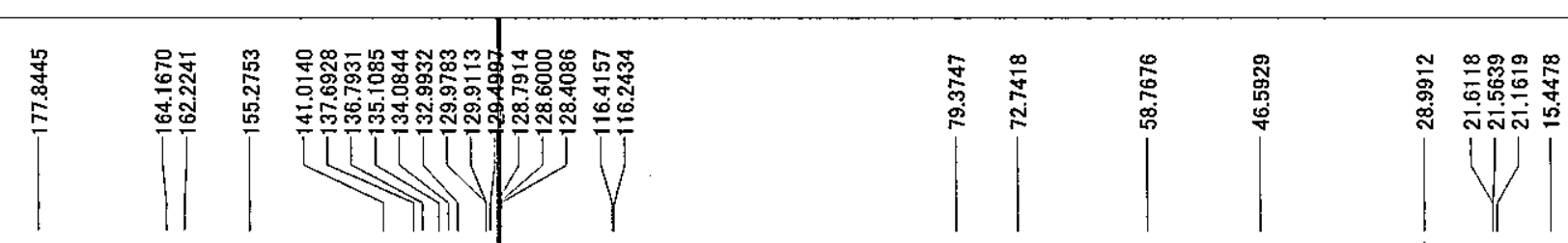

DFILE

COMN

DATIM

OBFRQ

OBSET

POINT

FREQU

SCANS

ACQTM

PD

IRNUC

SLVNT

EXREF

RFAIN

Documents and Settings $\because A l l$ Users $\because$ Docu Cingle pulse decoupled gated NOE

C NMR.ex 2

$3.45 \mathrm{KHz}$ $6.00 \mathrm{~Hz}$

$31249.52 \mathrm{~Hz}$

$0.8389 \mathrm{sec}$

$2.0000 \mathrm{sec}$
$3.67 \mathrm{usec}$

1H $25.5 \mathrm{c}$

C6D 6 $128.60 \mathrm{ppm}$

$1.20 \mathrm{ppm}$
$54 \mathrm{~Hz}$
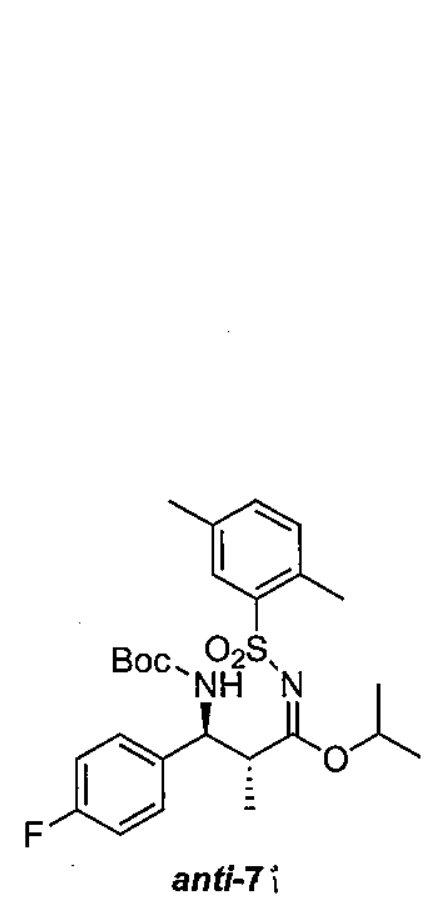

W 


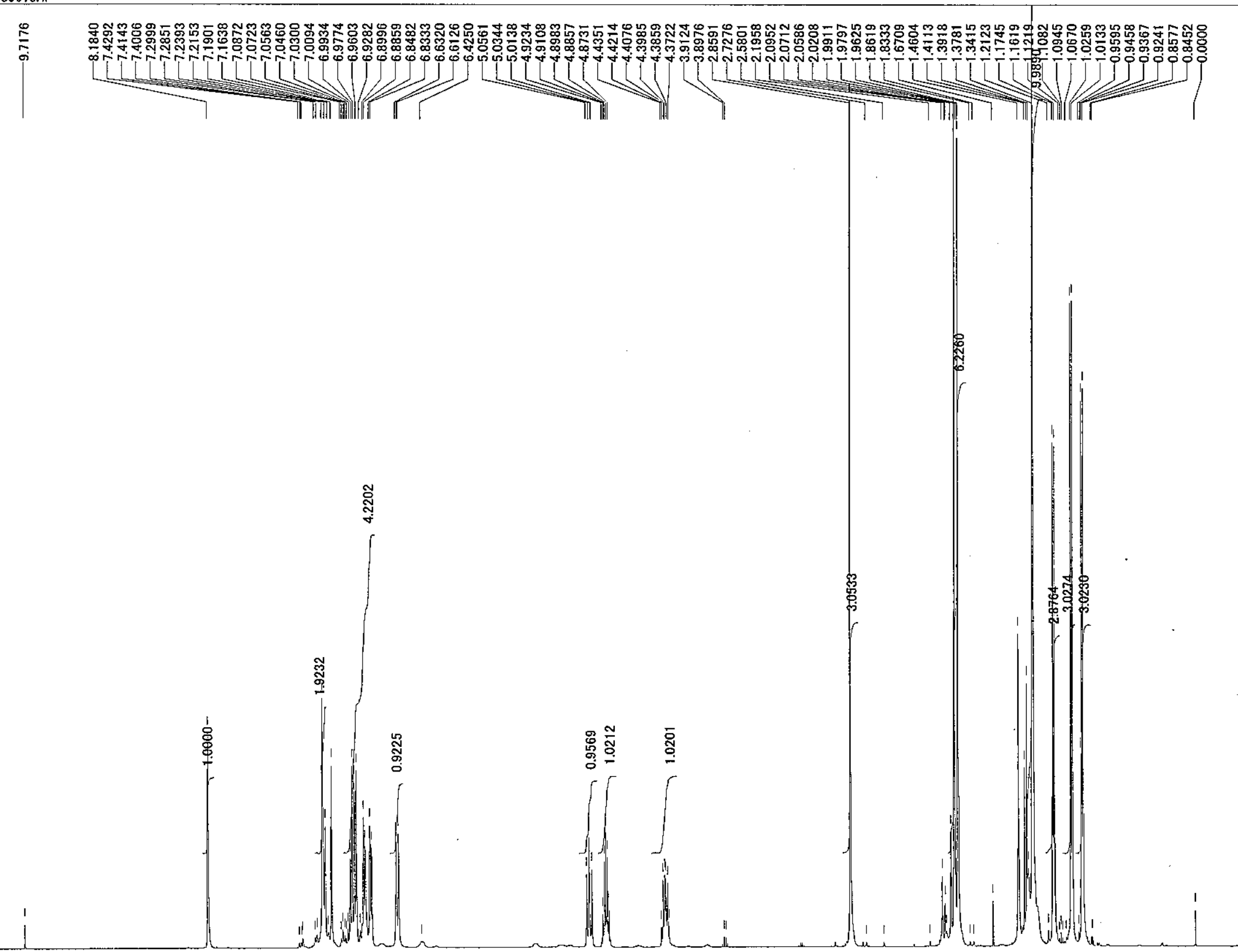

DFILE $\quad ¥ ¥$ Eca $¥$ data $¥$ florian¥¥ $\mathrm{fb} 396$ verif -1 .jdf

OBFRQ

OBSET

OBFIN

FREQU

SCANS

PD

IRNUC

CTEMP

SLVNT

BF

RGAIN

IH NMR.ex2

$495.13 \mathrm{MHz}$
$4.38 \mathrm{KHz}$

$9.64 \mathrm{~Hz}$

$9286.78 \mathrm{~Hz}$

8
$1.7642 \mathrm{sec}$

$5.0000 \mathrm{sec}$

$1 \mathrm{H} \quad 6.50$ use

$\mathrm{C} 6 \mathrm{D} 6{ }^{25.7 \mathrm{c}}$

$0.00 \mathrm{ppm}$
$0.12 \mathrm{~Hz}$
22

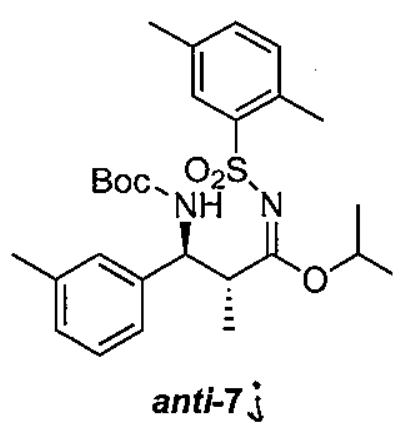




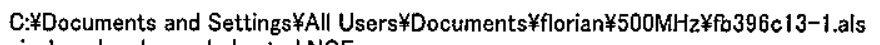

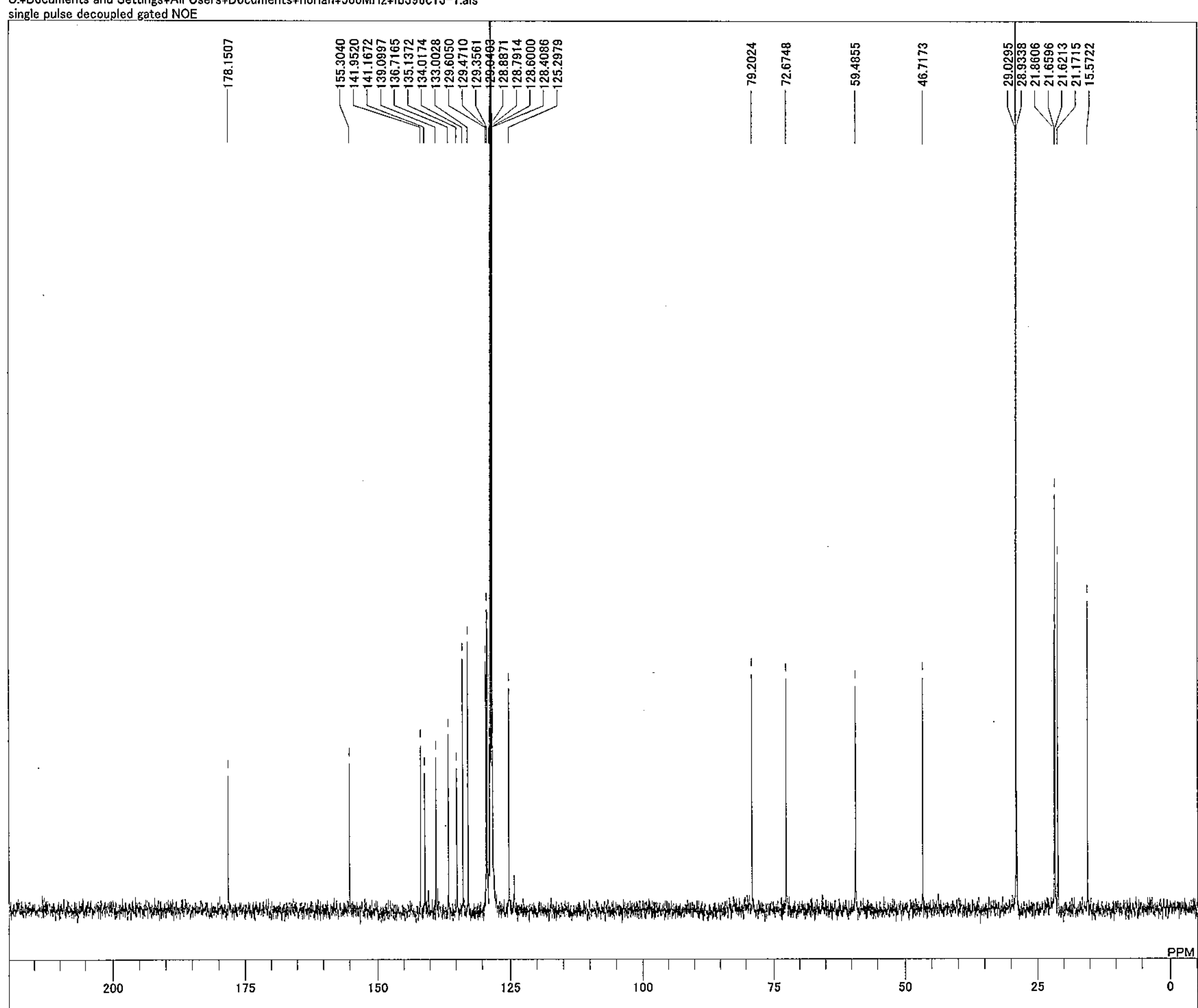

COMNT
DATIM

OBNUC

EXMOD

OBFRQ

OBFIN

SCANS

ACQTM

PD

IRNUC

CTEMP
SLVNT

EXREF

BF

RAIN
C:FDocuments and Settings:All Users $\#$ Docu Dulse decoupled gated NOE

$13 \mathrm{C}$ NMR.ex2 $124.51 \mathrm{MHz}$ $3.45 \mathrm{KHz}$ $6.00 \mathrm{~Hz}$ $31249.52 \mathrm{~Hz}$ $0.8389 \mathrm{sec}$ $2.0000 \mathrm{sec}$
3.67 usec

26.0 c C6D 6 $128.60 \mathrm{ppm}$ $128.60 \mathrm{ppm}$
$0.12 \mathrm{~Hz}$
50

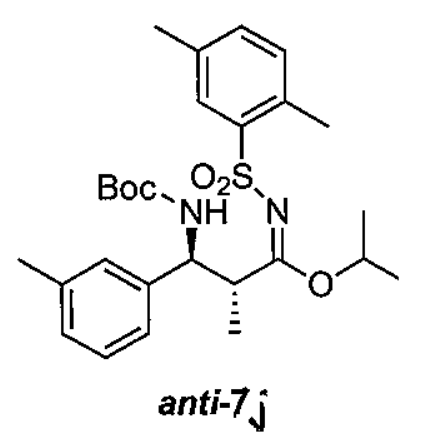




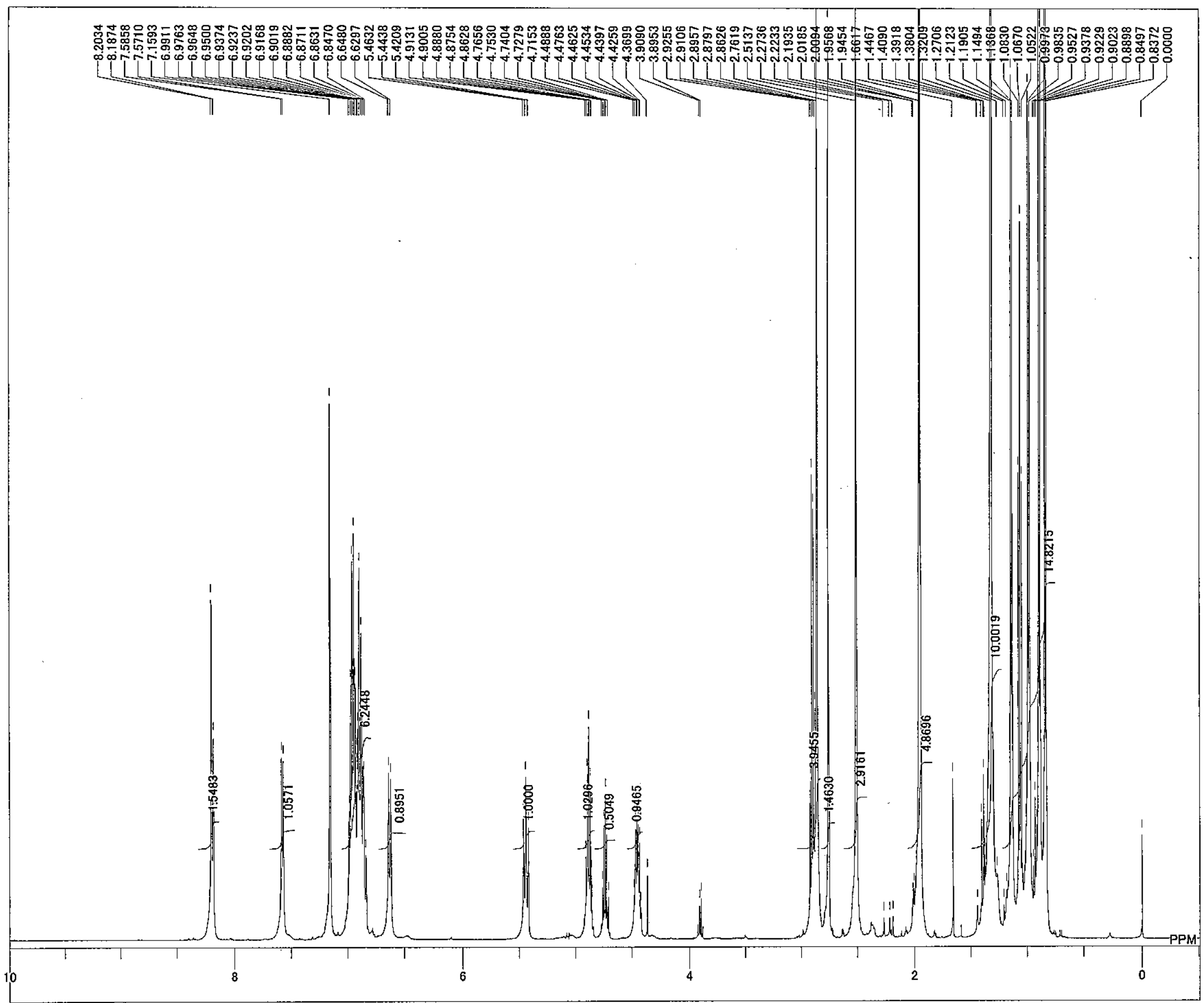

DFILE

DATIM

OBNUC

OBFRQ

OBSET

OBFIN

POINT

FREAUS
SCANS
ACQTM

ACQTM

PD 1

IRNUC

SLVNT

EXREF

RGAIN

rian羖394finalptlcdown-1.jof

0-07-2007 22:06:24

$1 \mathrm{H}$ NMR.ex2

$495.13 \mathrm{MHz}$
$4.38 \mathrm{KHz}$

$4.38 \mathrm{KHz}$
$9.64 \mathrm{~Hz}$

$288.78 \mathrm{~Hz}$

$9286.78 \mathrm{~Hz}$

$1.7642 \mathrm{sec}$
$5.0000 \mathrm{sec}$

$1 \mathrm{H} \quad 6.50 \mathrm{usec}$

${ }^{2} 6{ }^{26.3 \mathrm{c}}$

$0.00 \mathrm{ppm}$
$0.12 \mathrm{~Hz}$

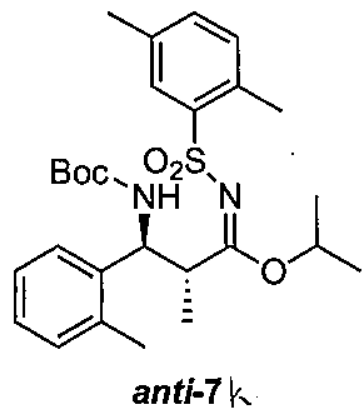


C: ¥Documents and Settings $¥ A$ All Users $¥$ Documents $¥$ florian $¥ 500 \mathrm{MHz} ¥ \mathrm{fb} 39413 \mathrm{c}-1$.als single pulse decoupled gated NOE
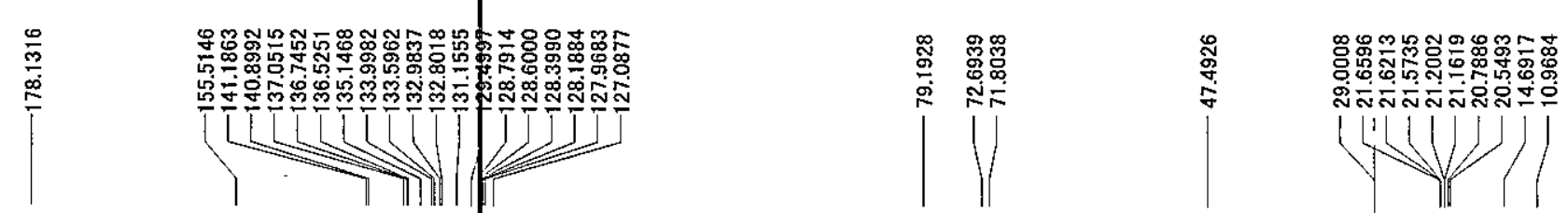

OBFRQ

OBSET

OBFIN
POINT
FREQU

FREQ

ACQTM

PD

IRNUC

SLVNT

EXREF

RGAIN

::FDocuments and Settings:All Users¥Docu ingle pulse decoupled gated NOE

$13 \mathrm{C}$ NMR,ex2

$124.51 \mathrm{MHz}$
$3.45 \mathrm{KHz}$ $3.45 \mathrm{KHz}$
$6.00 \mathrm{~Hz}$

$\frac{6.00 \mathrm{~Hz}}{26224}$

$31249.52 \mathrm{~Hz}$

$31249.52 \mathrm{~Hz}$
139
$0.8389 \mathrm{sec}$

$0.8389 \mathrm{sec}$
$2.0000 \mathrm{sec}$

3.67 usec

C606 ${ }^{26.7 \mathrm{C}}$

$128.60 \mathrm{ppm}$
$0.12 \mathrm{~Hz}$

$50 \mathrm{~Hz}$

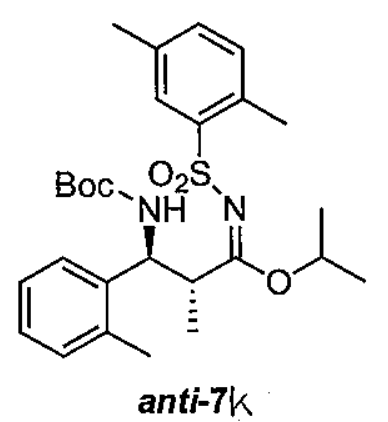

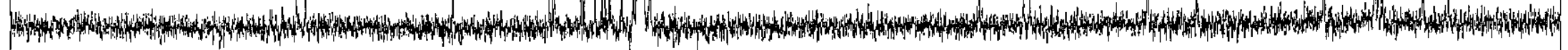

PPM

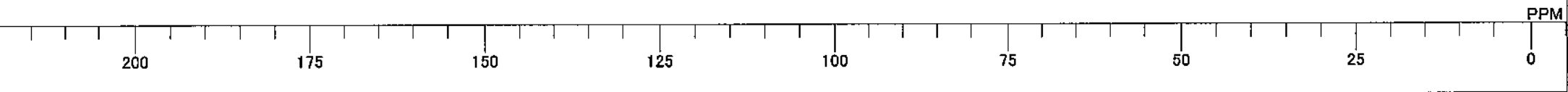




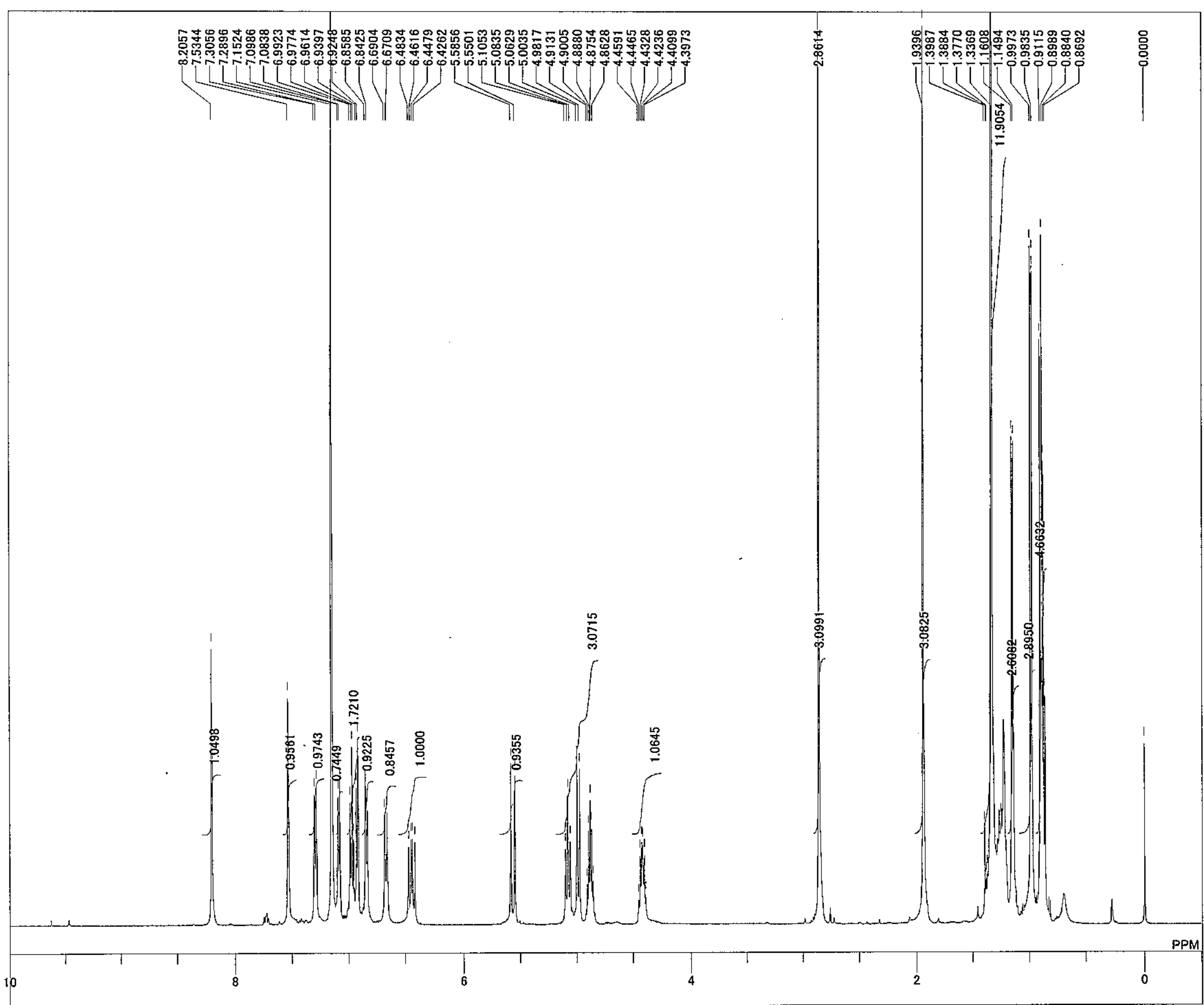

DFILE $\quad$ ¥Eca¥data¥florian¥fb383remade-3.jdf

DATIM

17:31:47

1H NMR.ex2
$495.13 \mathrm{MHz}$

$4.38 \mathrm{KHz}$

$9.64 \mathrm{~Hz}$

$9286.78 \mathrm{~Hz}$

$1.7642 \mathrm{sec}$

1 $\quad 6.50$ use

C6D6 $28.4 \mathrm{c}$

$0.00 \mathrm{ppm}$

BF

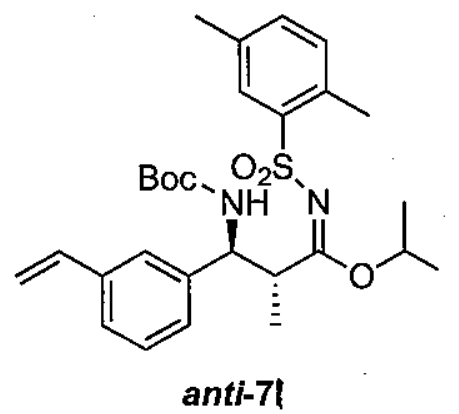



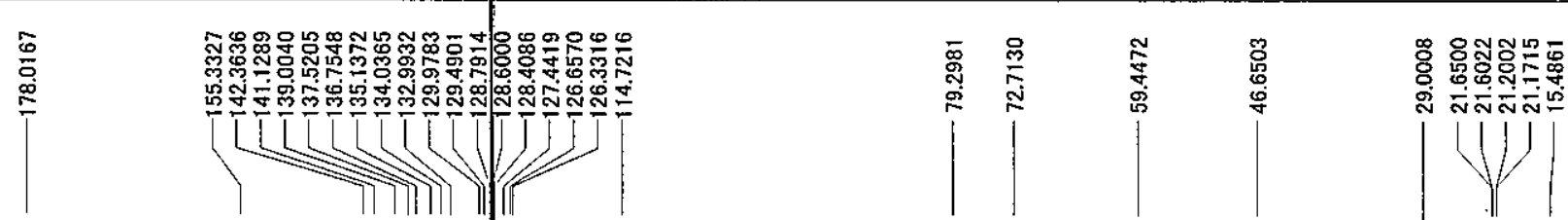

C:*Documents and Settings¥All Users¥Docu ingle pulse decoupled gated NOE

OBNUC

OBFRQ

OBSET

OBFIN

POINT

SCANS

ACQTM

PW 1

IRNUC

SLVNT
EXREF

EXREF

BF
RGAIN

$13 \mathrm{C}$ NMR.ex 2

$124.51 \mathrm{MHz}$
$3.45 \mathrm{KHz}$

26224
$31249.52 \mathrm{~Hz}$

65
$0.8389 \mathrm{sec}$

$2.0000 \mathrm{sec}$

$1 \mathrm{H} \quad 25.6 \mathrm{c}$

C6D 6 $128.60 \mathrm{ppm}$

$1.20 \mathrm{~Hz}$

54

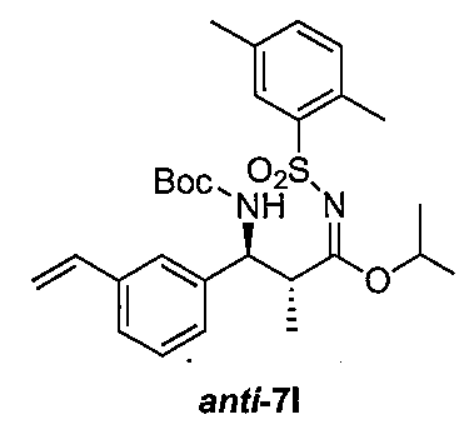

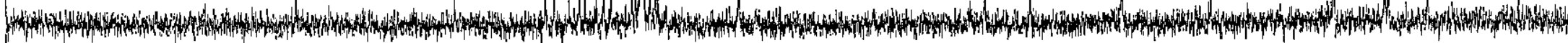




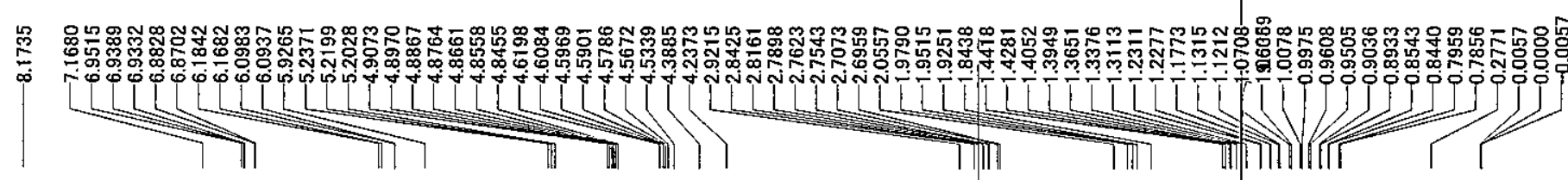

DFILE

C.7Documonts and Settings¥ALICE2¥デス

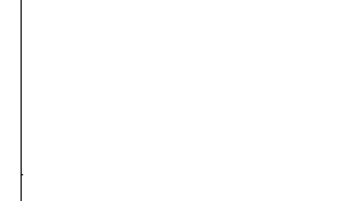

क

OATIM

EXMOD
OBFRQ
OBSET
OBFIN

POINT

FCANS

ACQTM

PW1

IRNUC

SLVNT

EXREF
BE
RER

BGAIN

$\mathrm{C} 6 \mathrm{D} 66^{29.4}$

${ }_{0.000 \mathrm{ppm}}^{\mathrm{C} 6 \mathrm{D} 6}$

$0.00 \mathrm{ppm}$
0.12
32

gle.pulse.ex2
$600.17 \mathrm{MHz}$

$5.30 \mathrm{KHz}$

$5.47 \mathrm{~Hz}$

20480

$1.4549 \mathrm{sec}$

$4.0000 \mathrm{sec}$
$9.10 \mathrm{usec}$
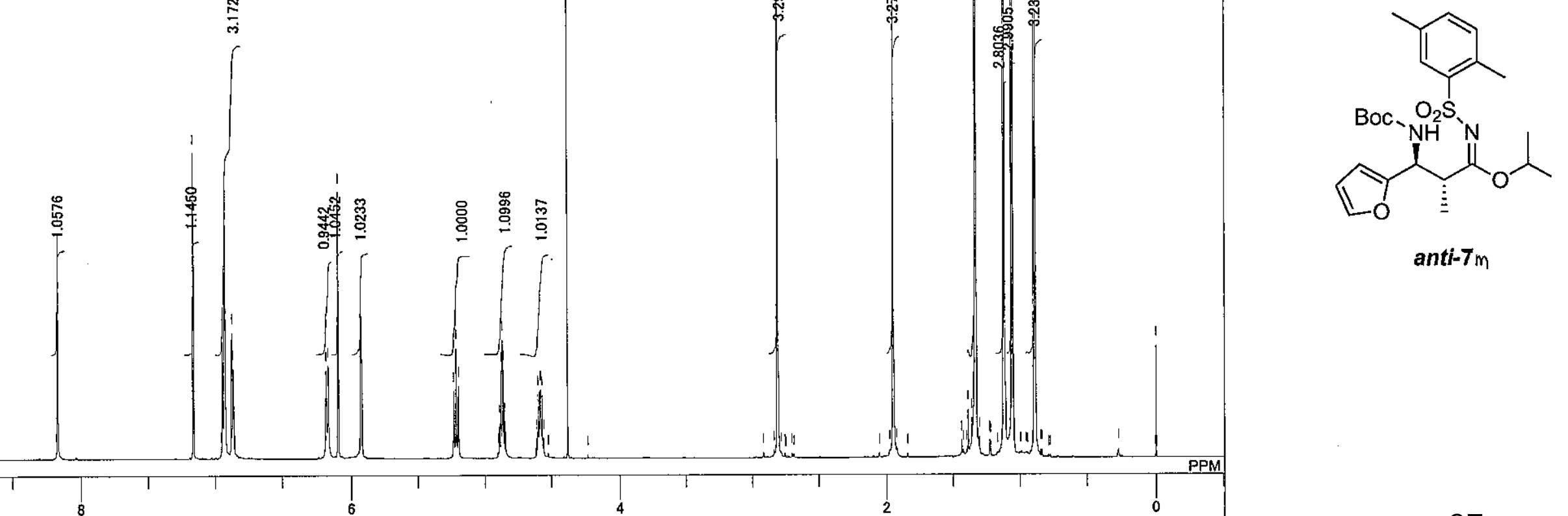
C: $¥$ Documents and Settings $¥$ All Users $¥ D$ ocuments $¥$ florian $¥ 500 \mathrm{MHz} ¥ \mathrm{fb} 381 \mathrm{c} 13 \mathrm{pp}-1$. als single pulse decoupled gated NOE

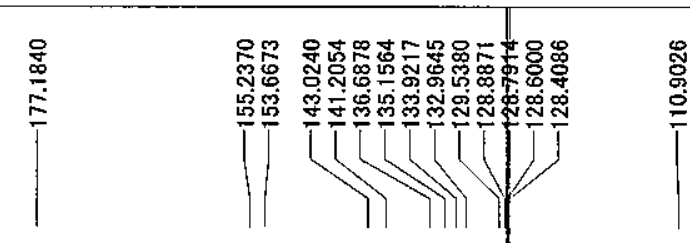

$\mid$
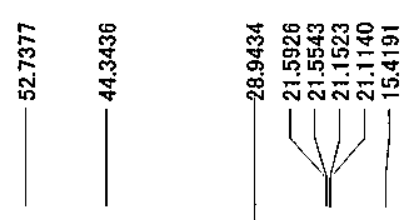

DFILE

DATIM EXMOD

OBFRQ

OBSET

OBFIN

FREQU

SCANS

$P D$

PW1

CTEMP
SLVNT

EXREF

RF

C:*Documents and Settings:All Users $¥$ Docu single pulse decoupled gated NOE

13C NMR.ex2

$$
\begin{gathered}
124.51 \mathrm{MHz} \\
3.45 \mathrm{KHz}
\end{gathered}
$$

$2.00 \mathrm{~Hz}$

26224
$31249.52 \mathrm{~Hz}$

62
$0.8389 \mathrm{sec}$

$2.0000 \mathrm{sec}$

IH $25.5 \mathrm{c}$

$\mathrm{C} 6 \mathrm{D} 6$
$128.60 \mathrm{ppm}$

$128.60 \mathrm{ppm}$
$1.20 \mathrm{~Hz}$

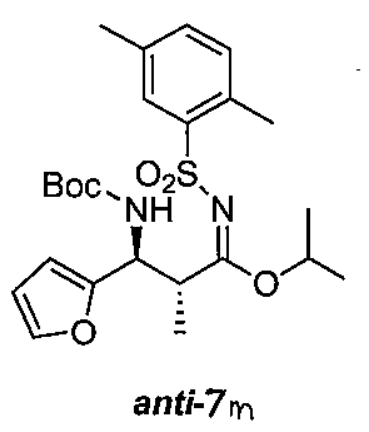

Atw 


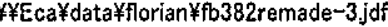

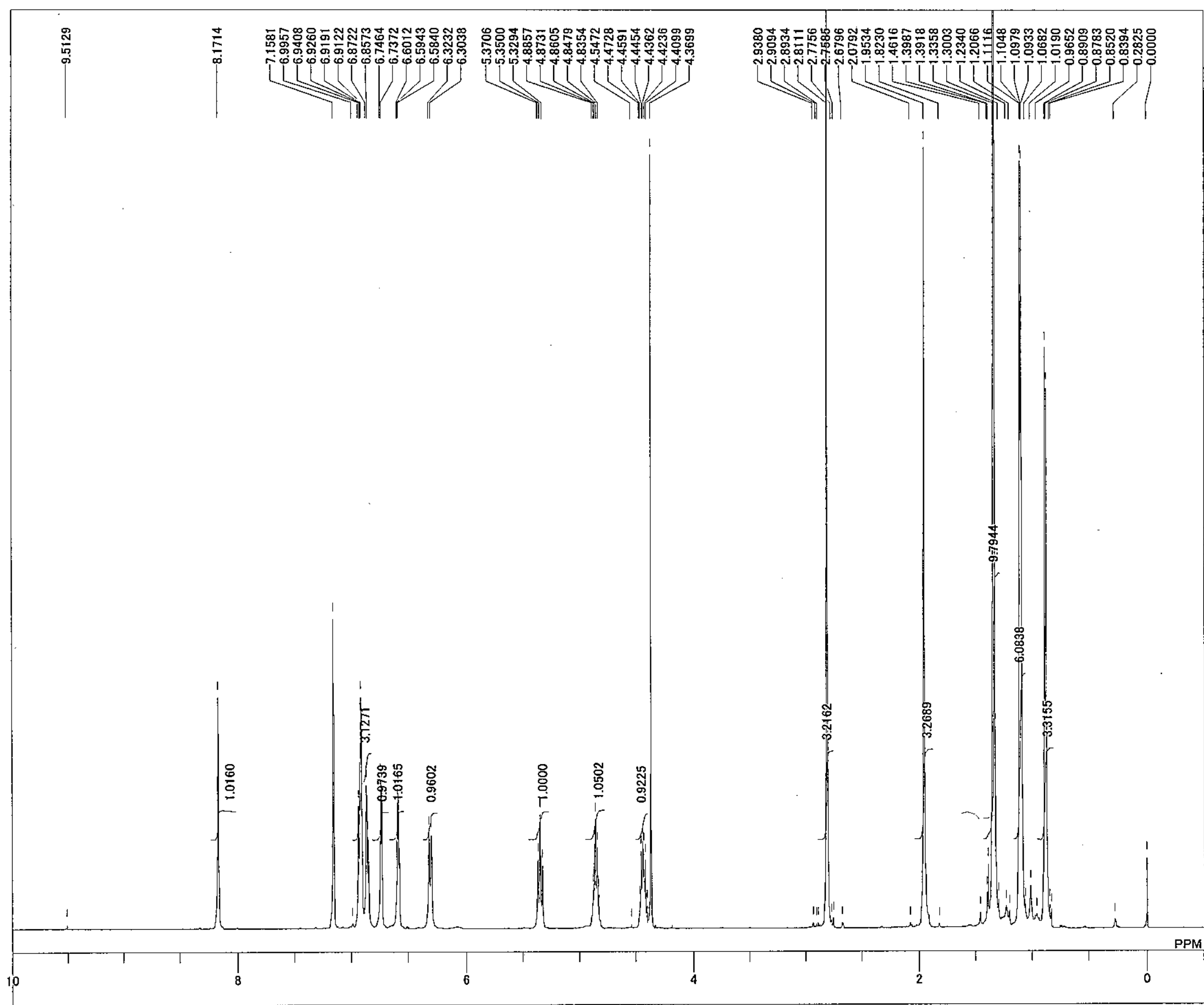

DFILE

COMN

OBNUC

OBSET

OBFIN

FREQU

SCANS

PD

PW1

EXREF

RGAIN

Eca $¥$ data $¥$ florian $¥$ fb 382 remade $-3 . j$ df

29-08-2007 20:51:07

IH NMR.ex2

$495.13 \mathrm{MHz}$
$4.38 \mathrm{KHz}$

$9.64 \mathrm{~Hz}$

$9286.78 \mathrm{~Hz}$

8

$1.7642 \mathrm{sec}$

$5.0000 \mathrm{sec}$

$1 \mathrm{H} 2820$

$\mathrm{C} 6 \mathrm{D} 6$

$0.00 \mathrm{ppm}$
$0.12 \mathrm{~Hz}$

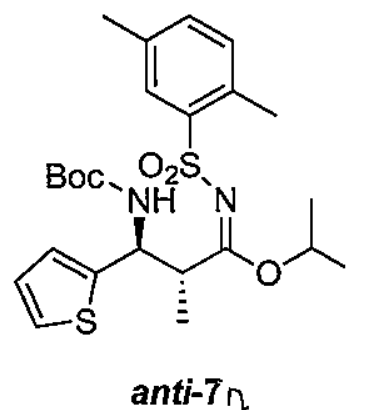


C: $:$ Documents and Settings $¥$ All Users $*$ Documents $¥$ florian $¥ 500 \mathrm{MHz} ¥ \mathrm{fb} 382 \mathrm{c} 13 \mathrm{pp}-1$. als

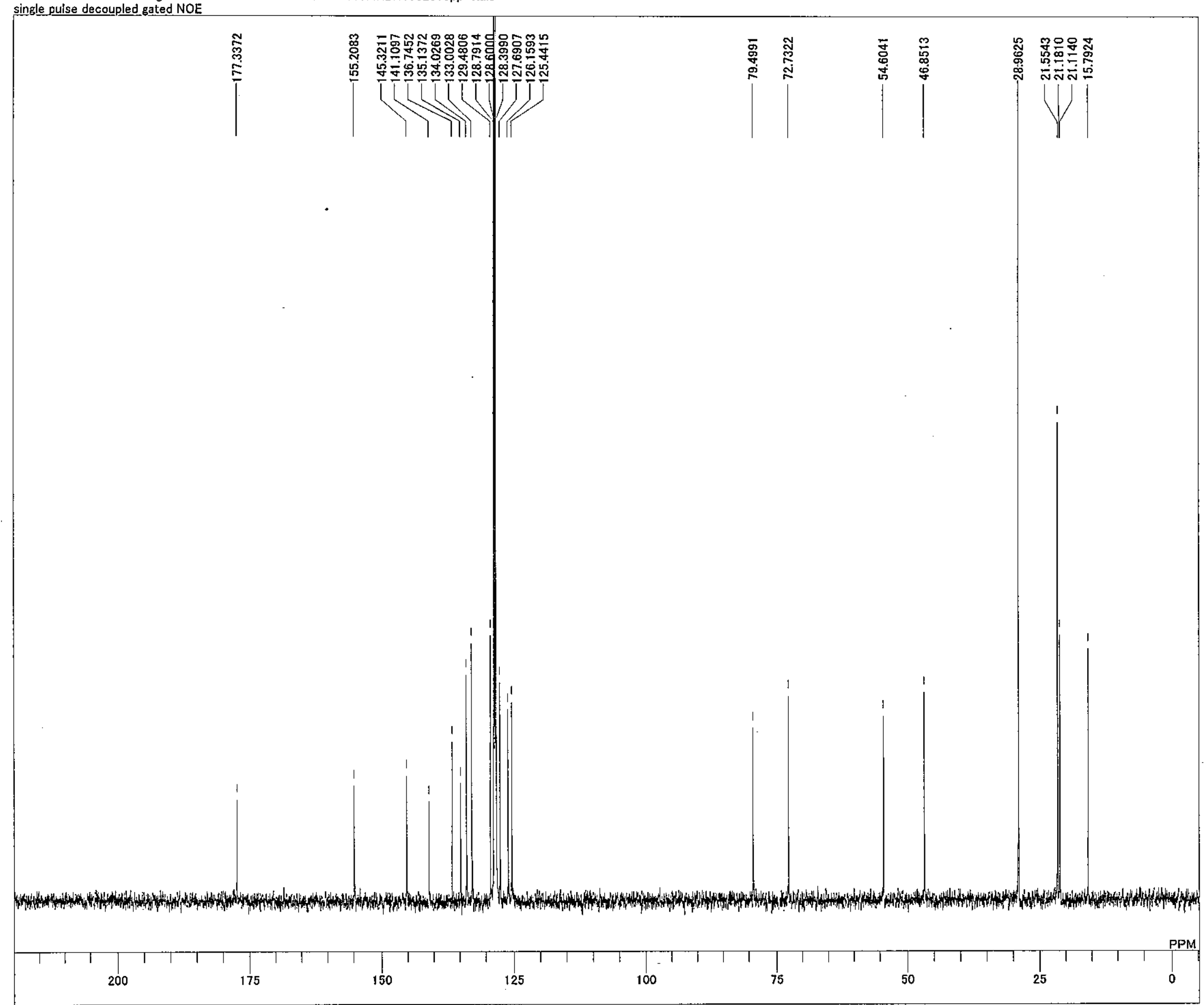

DFILE

DATIM

OBNUC

EXMOD

OBSET

BFiN

FREQU

SCANS

ACQTM

PW1

IRNUC

SLVNT

EXREF

RGAIN

C6D $6{ }^{25 .}$

$128.60 \mathrm{ppm}$

$1.20 \mathrm{~Hz}$
52

attings:All Users¥Doc gle pulse decoupled gated NOE

13C NMR.ex2 $124.51 \mathrm{MHz}$ $3.45 \mathrm{KHz}$ $6.00 \mathrm{~Hz}$ $31249.52 \mathrm{~Hz}$

$0.8389 \mathrm{sec}$

$2.0000 \mathrm{sec}$

.67 usec

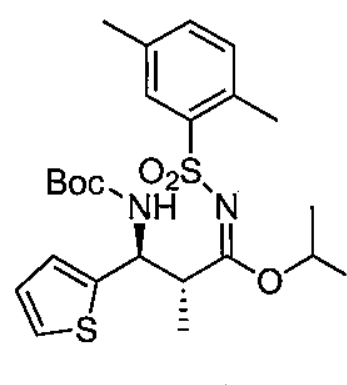

anti-7n 


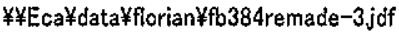

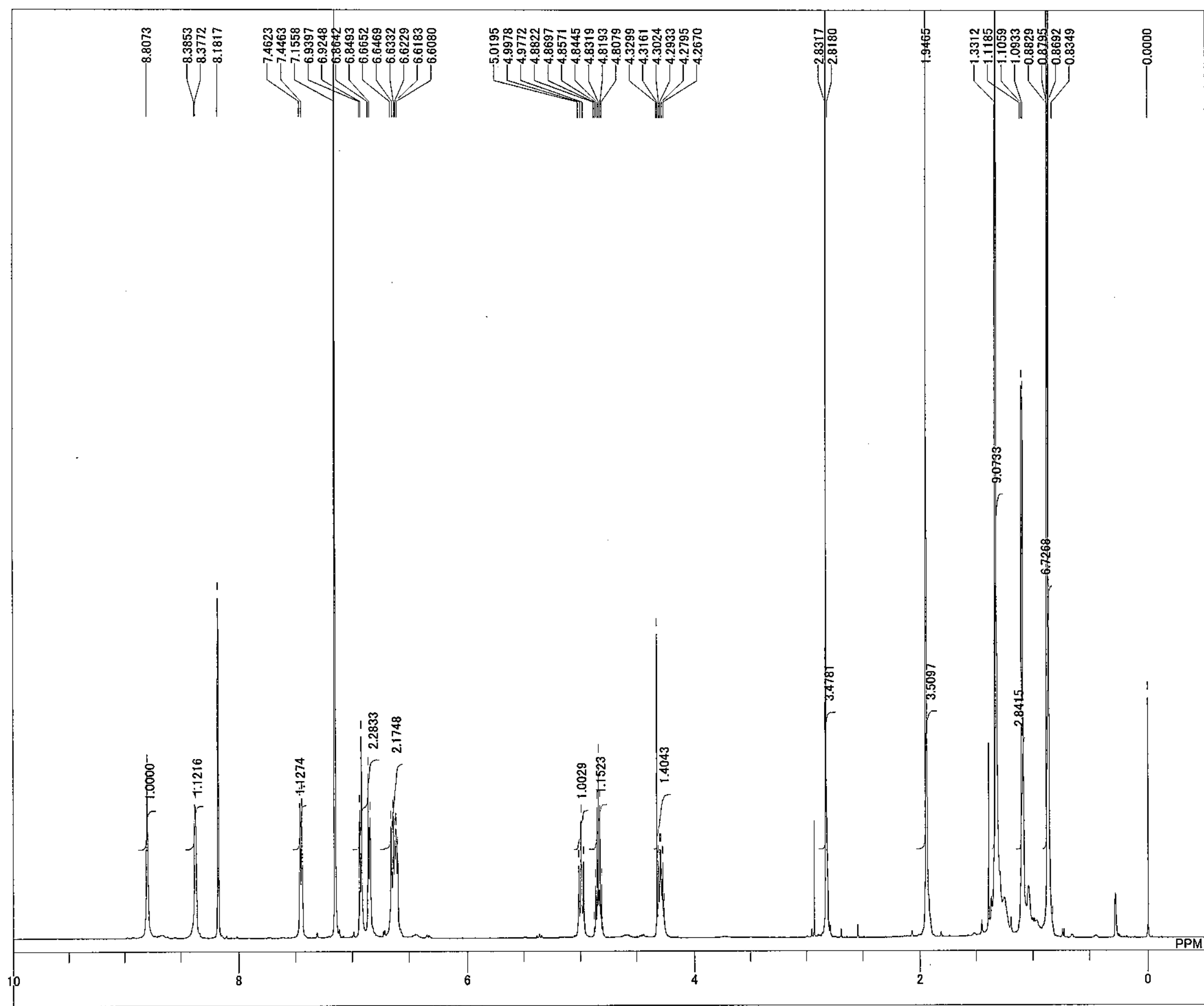

DFILE $¥ ¥$ Eca $¥$ data $¥$ florian $¥ \mathrm{fb} 384$ remade $-3 . j \mathrm{df}$

DATIM 29-08-2007 17:41:08

THM.13 $\mathrm{MHz}$

$4.38 \mathrm{KHz}$

$9.64 \mathrm{~Hz}_{2}$

$9286.78 \mathrm{~Hz}$

FREQU 928

ACQTM

$1.7642 \mathrm{sec}$

$6.50 \mathrm{sec}$

$1 \mathrm{H} 6.50 \mathrm{ds}$

CTEMP $28.5 \circ$

SLVNT

EXREF

$\mathrm{C} 6 \mathrm{D6}{ }^{28.5}$

$0.00 \mathrm{ppm}$
$0.12 \mathrm{~Hz}$
36

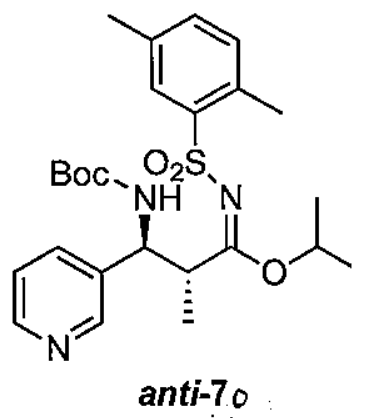


C: $¥ D$ ocuments and Settings $¥ A$ All Users $¥ D$ Documents $¥$ florian $¥ 500 \mathrm{MHz} ¥ \mathrm{Fb} 384 \mathrm{c} 13 \mathrm{pp}-1$.als single pulse decoupled gated NOE

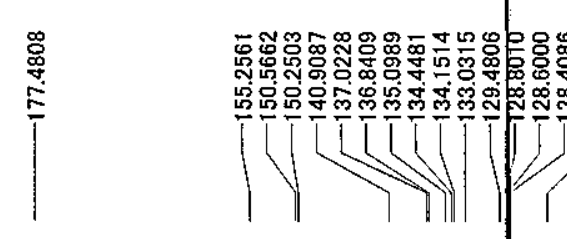

$\mid$ |

DFILE

DATIM

OBNUC

EXMOD

OBFRQ

OBSET

POINT

FREQU

SCANS

ACQTM

PWI

IRNOM

SLVNT

$\underset{B F}{\text { EXREF }}$

RGAIN
C:FDocuments and Settings:All Users $¥$ Docu ingle pulse decoupled gated NOE

13C NMR.ex2

$124.51 \mathrm{MHz}$ $3.45 \mathrm{KHz}$

26224

$0.8389 \mathrm{sec}$

$2.0000 \mathrm{sec}$
$3.67 \mathrm{usec}$

25.60

$\mathrm{C} 6 \mathrm{D} 6$
$128.60 \mathrm{ppm}$ $128.60 \mathrm{ppm}$
t. $20 \mathrm{~Hz}$
54

W.

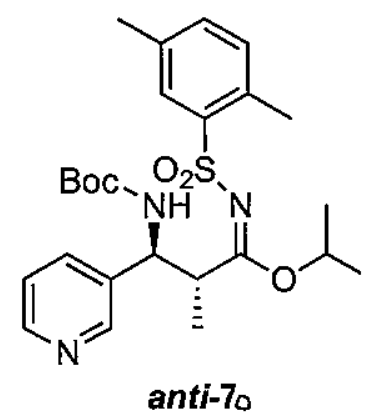




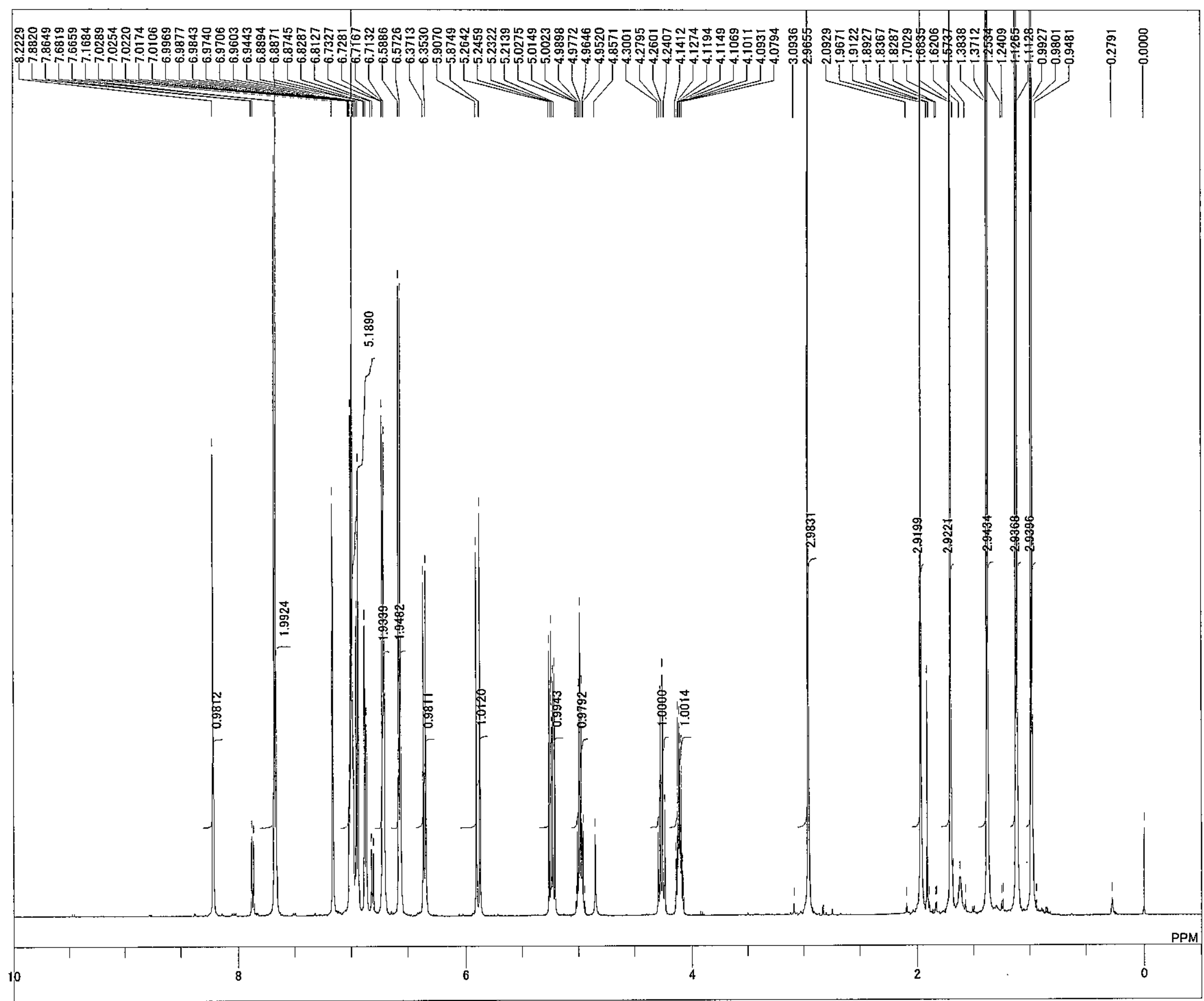

DFILE

DATIM

EXMOD

EXMOD

OBSET

OBFIN

FREQU

SCANS

PD

PW1

CTEMP

SLVNT

EXREF

RGAIN

08-08-2007 20:29:43

H NMR.ex2

$4.38 \mathrm{KHz}$

$9.64 \mathrm{~Hz}$

$9286.78 \mathrm{~Hz}$

8
$1.7642 \mathrm{sec}$

$5.0000 \mathrm{sec}$

6.50 use

27.9 。

$0.12 \mathrm{~Hz}$

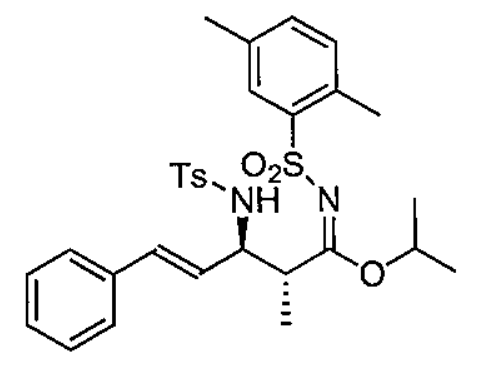

anti-7p 
C:FDocuments and Settings $¥ A$ All Users $¥ D$ ocuments $¥$ florian $¥ 500 \mathrm{MHz} ¥ \mathrm{fb} 442 \mathrm{c} 13-1 . \mathrm{als}$ single pulse decoupleo gated NOE
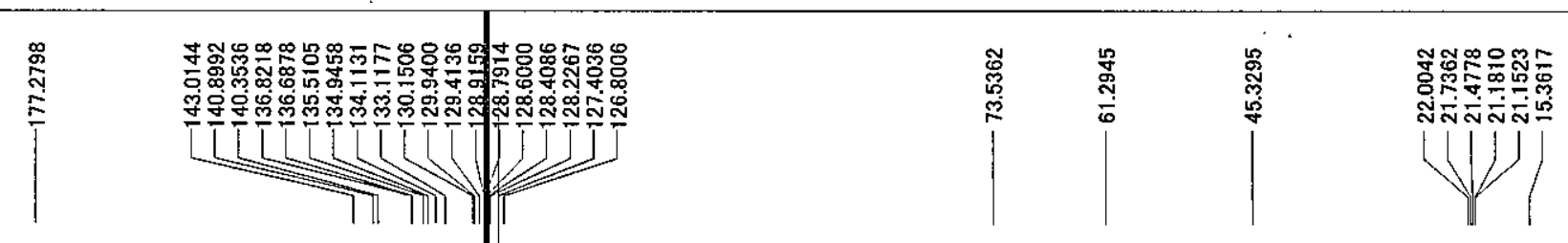

DFILE

DATIM

OBNUC

EXMOD

OBFRO

OBSET

POINT

$\quad 31249.52 \mathrm{~Hz}$

$\begin{array}{ll}\text { SCANS } & 64 \\ \text { ACQTM } & 0.8389 \mathrm{sec}\end{array}$

PD $\quad 2.0000 \mathrm{sec}$

PW1
IRNUC $1 \mathrm{H} \quad 3.67$ use

CTEMP $28.2 \mathrm{c}$

$\begin{array}{ll}\text { SLVNT } & \text { C6D } 6 \\ \text { EXREF } & 128.60 \mathrm{ppm}\end{array}$

BF

$0.12 \mathrm{~Hz}$
54

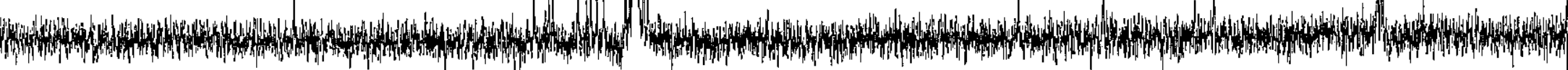

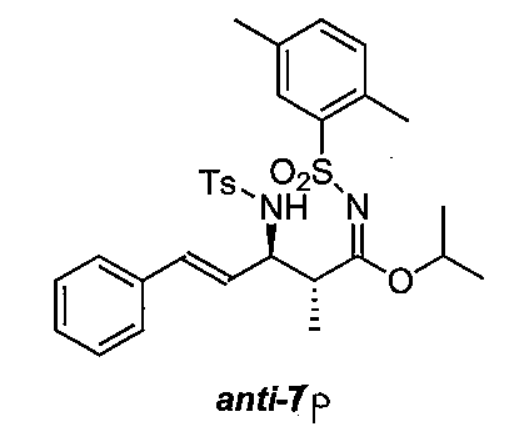

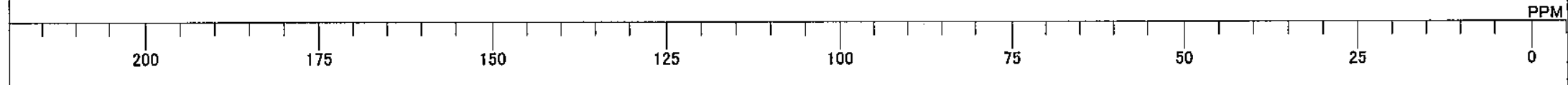




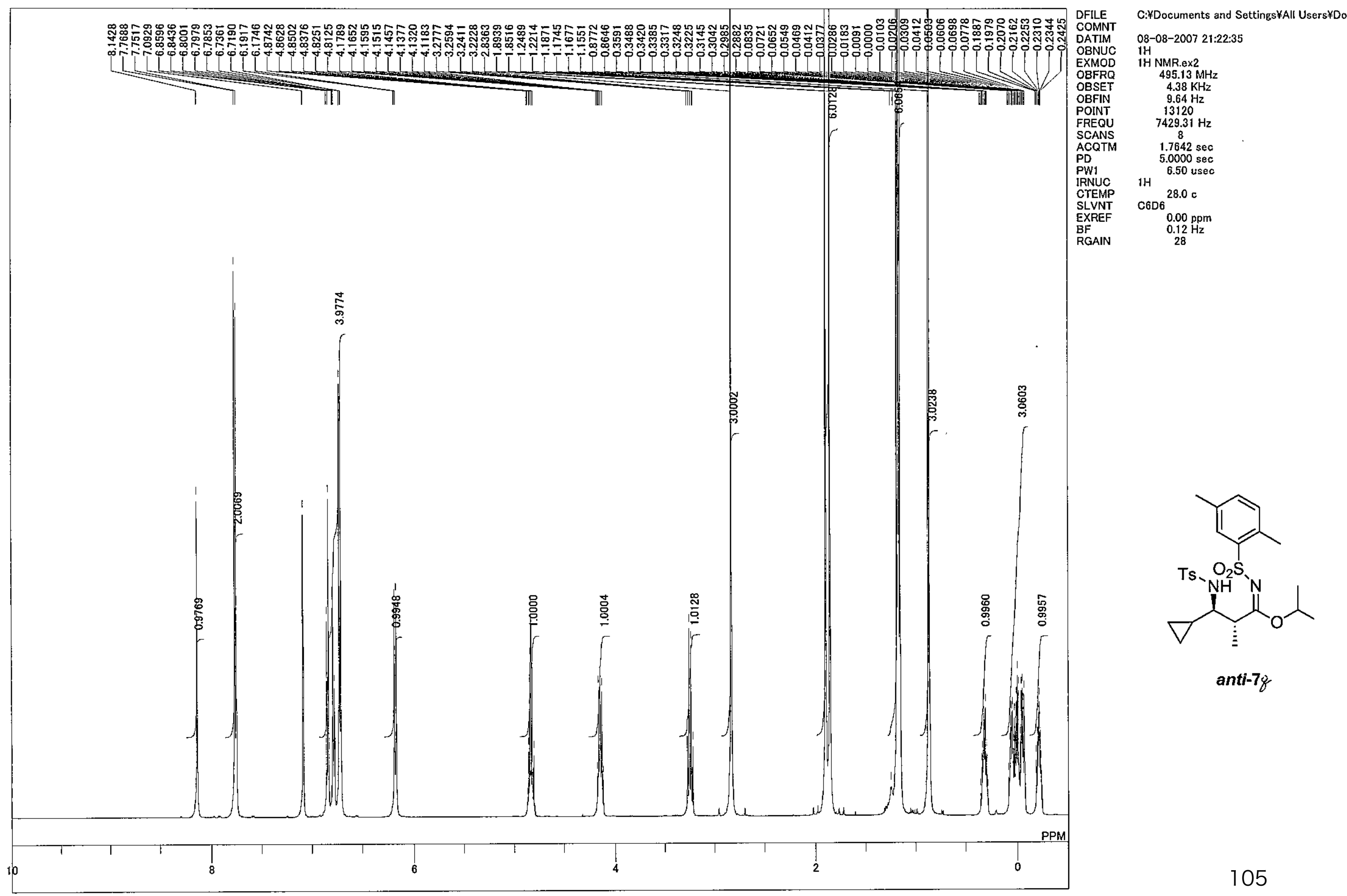



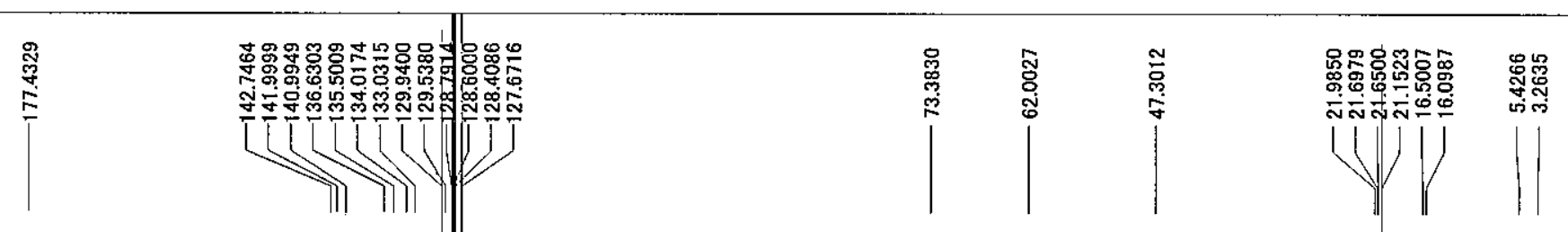

C:*Documents and Settings

DATIM

EXMOD

OBFRQ

OBSET

POINT

FREQU

SCANS

ACQT:

PW1

IRNUC

SLVNT

EXREF

RGAIN

pulse decoupled gated NOE

$13 \mathrm{C}$ NMR.ex2

$3.45 \mathrm{KHz}$
$6.00 \mathrm{~Hz}$

$6.00 \mathrm{~Hz}$

$31249.52 \mathrm{~Hz}$

62

$0.8389 \mathrm{sec}$
$2.0000 \mathrm{sec}$

$2.0000 \mathrm{sec}$
$3.67 \mathrm{usec}$

$\mathrm{C}^{2}{ }^{28.3 \mathrm{C}}$

$128.60 \mathrm{ppm}$
$0.12 \mathrm{~Hz}$
50

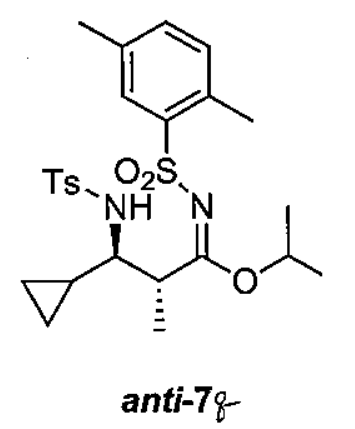

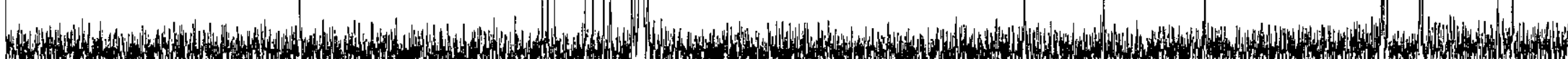
M 
C: $¥$ Documents and Settings $¥$ delta $¥ M y$ Documents $*$ Personal Folder¥Matsubara $¥ 2801-2900 ¥ 2831$ up.als

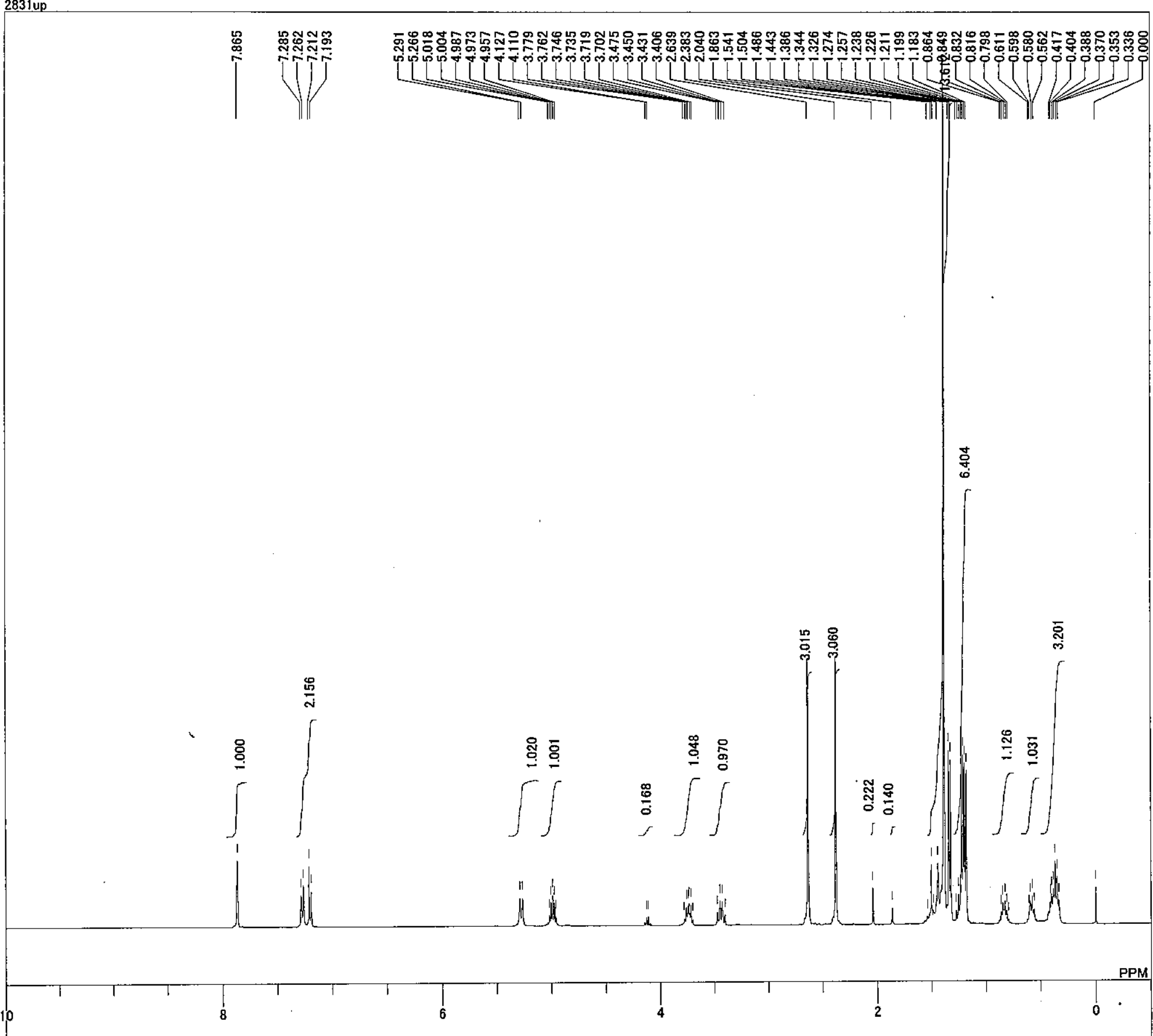

DFILE

COMNT
DATIMT

DATIM

EXMOD
OBFRQ
OBSET

OBFRQ
OBSET
OBFIN
POINT

FREQU

SCANS

PD

PW1

CTEMP

SLVNT
EXREF
BF

BF

Cocuments and Settings $¥$ delta $¥ M y$ Documents $\$$ Perso

3-12-2007 20:23:47

ingle.pulse.ex2

399.78
$4.19 \mathrm{MHz}$

$7.29 \mathrm{~Hz}$

$7503.00 \mathrm{~Hz}$
8

$2.1837 \mathrm{sec}$
$2.0000 \mathrm{sec}$

5.90 usec

$25.0 \circ$

$0.00 \mathrm{ppm}$
$0.12 \mathrm{~Hz}$ 
C:FDocuments and Settings $¥ A$ All Users $¥$ Documents $¥$ Matsubara $¥ 2801-2900 * 2831$ upC.als

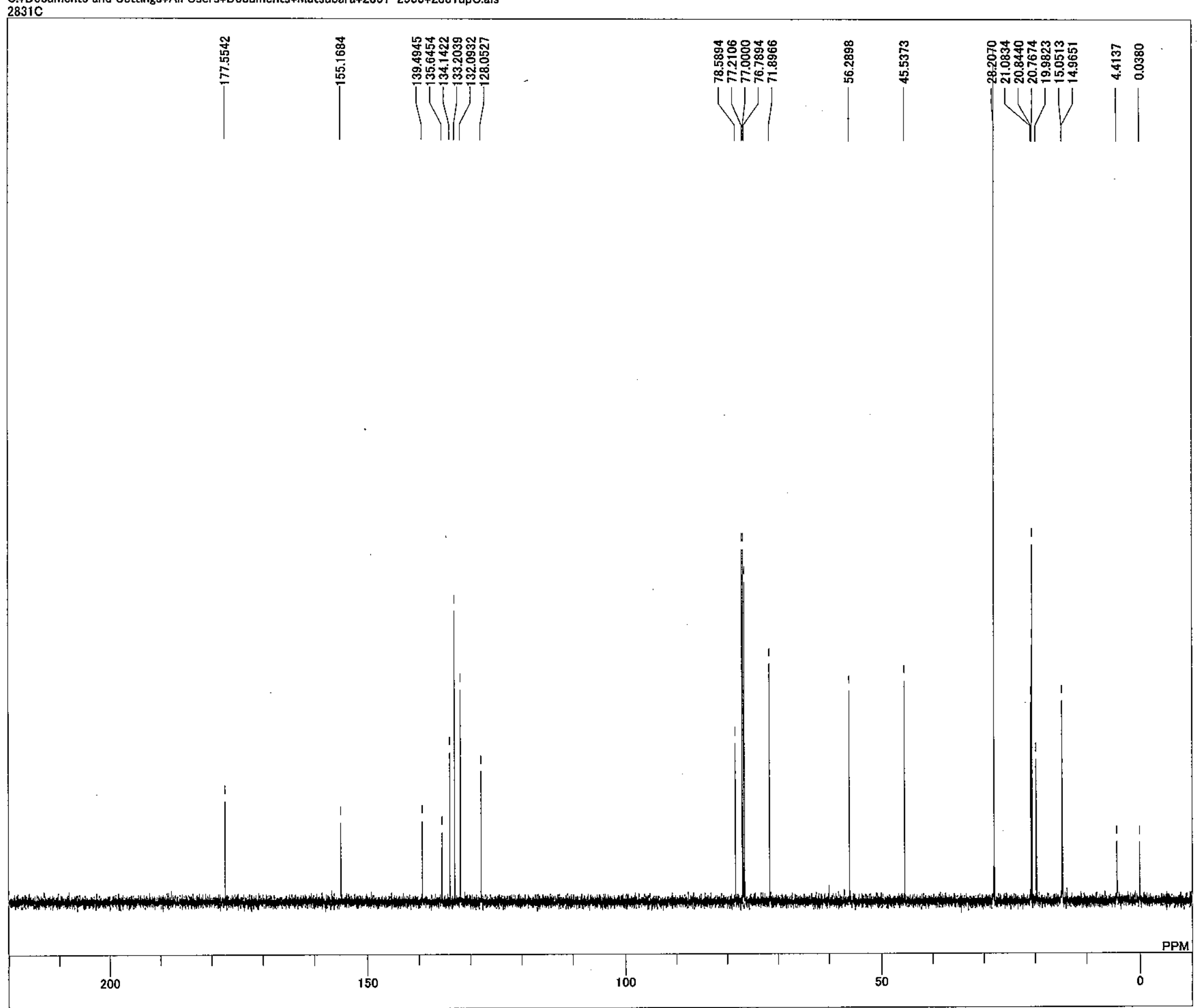

DFILE C:FDocuments and Settings:All Users¥Docu

$\begin{array}{ll}\text { COMNT } & 2831 \mathrm{C} \\ \text { DATIM } & 13-12-200720: 55: 28\end{array}$

OBNUC

$\begin{array}{ll}\text { EXMOD } & \text { single.pulse_dec } \\ \text { OBFRQ } & 150.92 \mathrm{MHz} \\ \text { OBSE } & 8.52 \mathrm{KHz}\end{array}$

OBSET $\quad 8.52 \mathrm{KHz}$

OBFIN

$1.74 \mathrm{~Hz}$
26214
$37878.21 \mathrm{~Hz}$

$37878.21 \mathrm{~Hz}$

$\begin{array}{lc}\text { SCANS } & 61 \\ \text { ACQTM } & 0.6921 \mathrm{sec}\end{array}$

$\begin{array}{ll}\text { ACQTM } & 0.6921 \mathrm{sec} \\ \mathrm{PD} & 2.0000 \mathrm{sec}\end{array}$

PW1 2.83 usec

IRNUC $1 \mathrm{H} \quad 22.6 \mathrm{c}$

SLVNT $C D C L 33$

$\begin{array}{lc}\text { EXREF } & 77.00 \mathrm{ppm} \\ \text { BF } & 0.12 \mathrm{~Hz} \\ \text { RGAIN } & 58\end{array}$

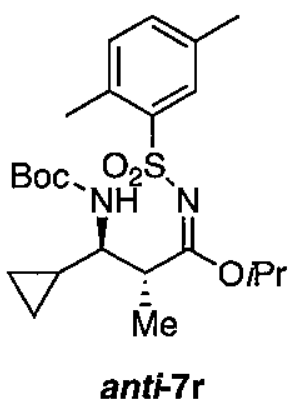




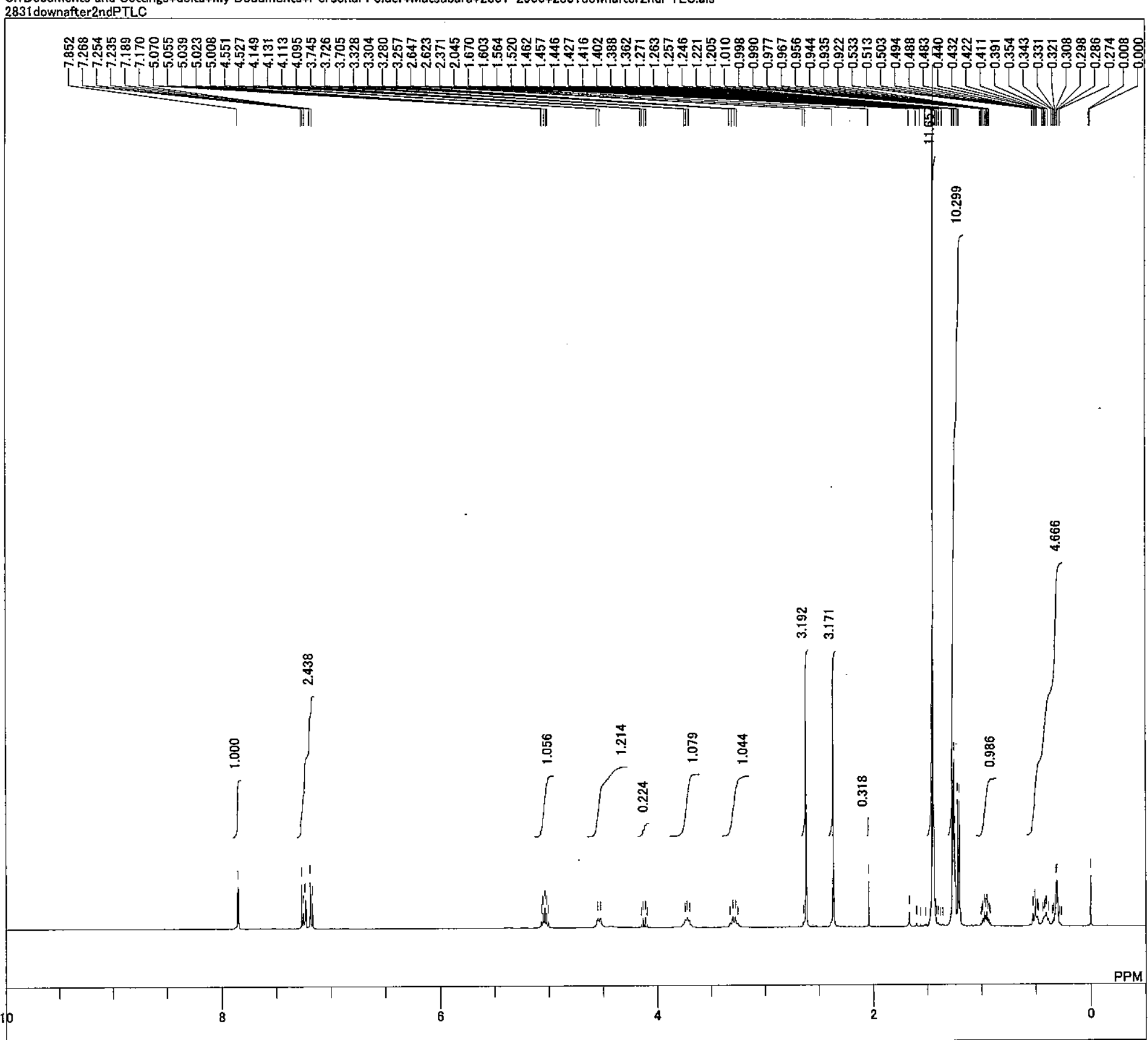

DFILE

DATIM

EXMOD

OBSET

OBFIN

POINT

SCANS

ACQT

PW1

SLVNT

EXREF

RGAIN

Documents and Settings $¥$ delta $¥ M y$ Documents*Perso 4-12-2007 19:11:56

single.pulse.ex2 $4.19 \mathrm{KHz}$ $7.29 \mathrm{~Hz}$ $6002.31 \mathrm{~Hz}$ 8
$2.1837 \mathrm{sec}$
$2.0000 \mathrm{sec}$ $2.0000 \mathrm{sec}$
$5.90 \mathrm{usec}$ 25.0 $0.00 \mathrm{ppm}$
$0.12 \mathrm{~Hz}$
32

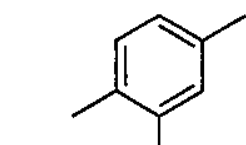
Boc $\mathrm{O}_{2} \mathrm{~S}$

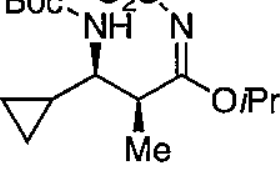
syn-7r 
C:FDocuments and Settings*All Users*Documents*Matsubara*2801-2900*2831 down-C_CARBON.3

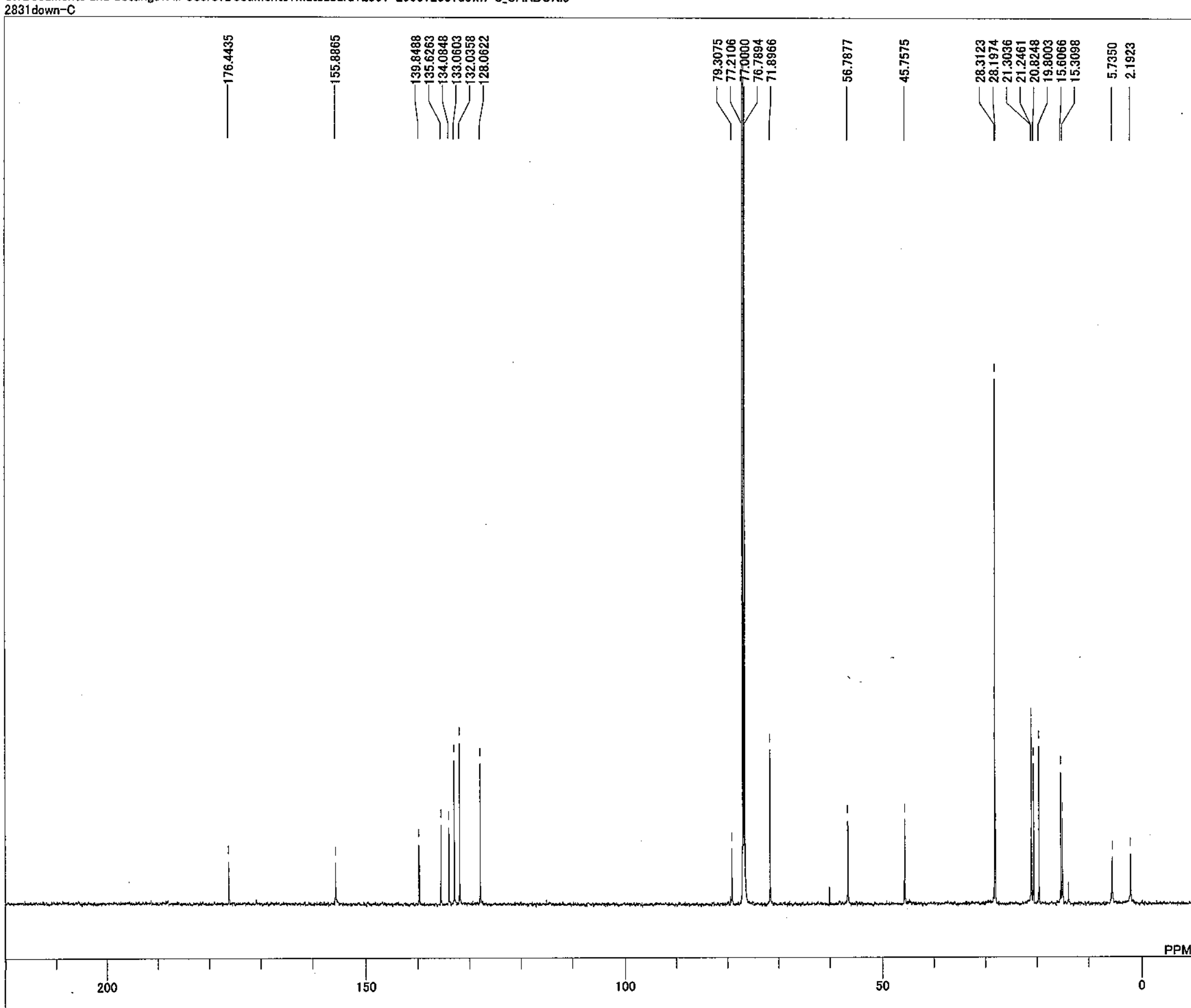

DFILE C:FDocuments and Settings*All Users*Docu

DATIM

OBFRQ

OBSET

OBFIN

FREQU

SCANS

ACOTM

PD

PW1

CTEMP

SLVNT

EXREF

RGAIN

-12-2007 10:27:37

ingle pulse_dec $8.52 \mathrm{KHz}$ (.74

$59186.51 \mathrm{~Hz}$

3000

$0.6921 \mathrm{sec}$

$2.0000 \mathrm{sec}$

$1 \mathrm{H}$

$\operatorname{CDCL}^{23} 3$

$77.00 \mathrm{ppm}$
$0.12 \mathrm{~Hz}$

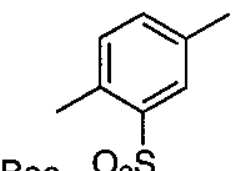

Boc $\mathrm{O}_{2} \mathrm{~S}$

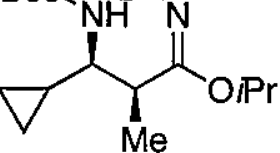

syn-7r 


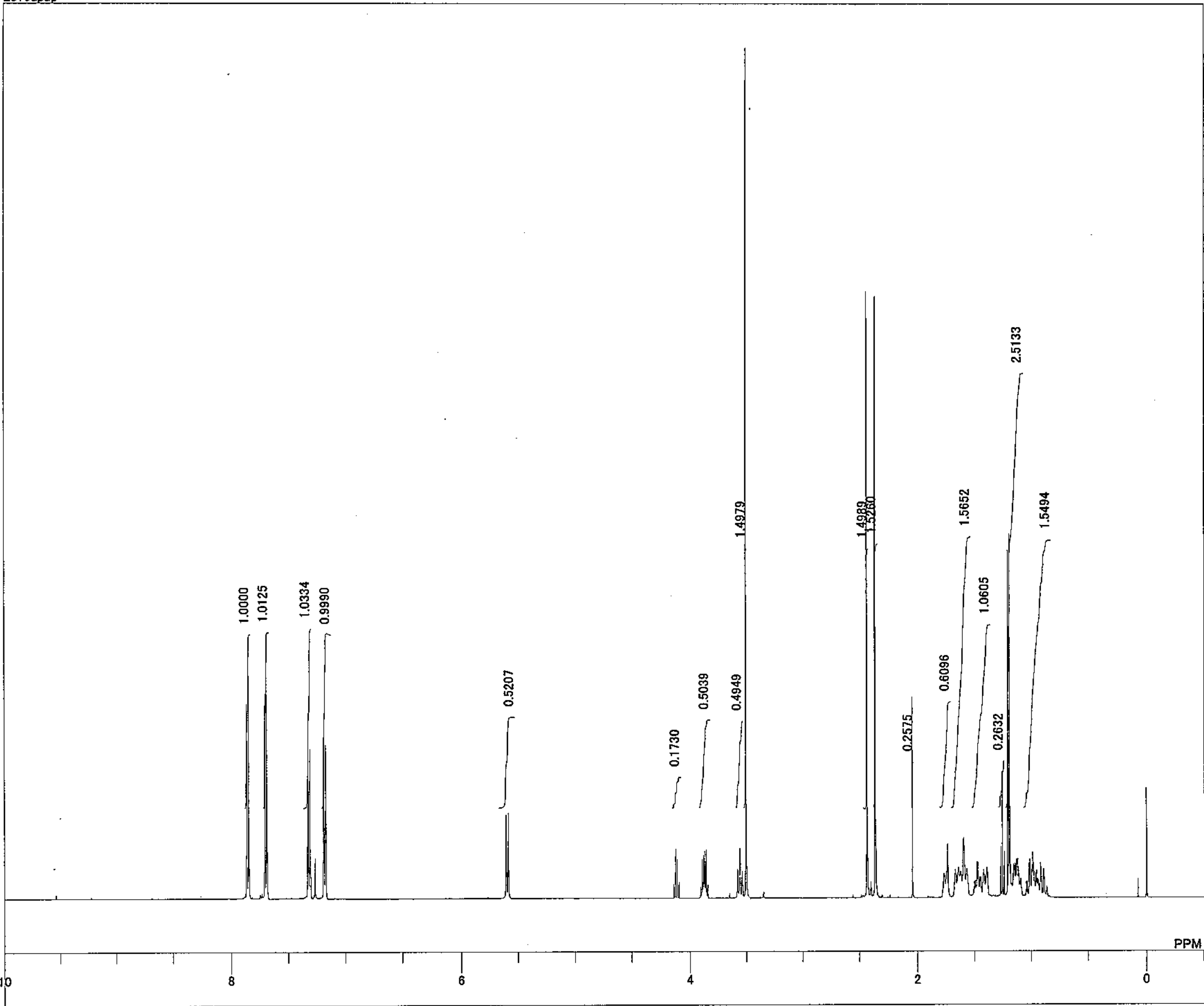


DFILE

OBSET

OBFIN

POINT

SCANS

ACQTI
PD

PD 1

PWN1

CTEMP

SLVNT

EXREF

RGAIN

AIN

$58 \mathrm{~Hz}$

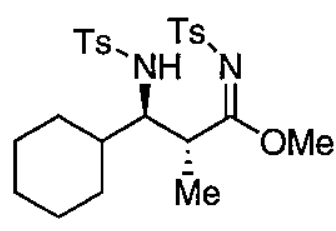

$a n+i-7 s$ 
DFILE $\quad$ C:FDocuments and Settings $\# A l l$ Users $¥$ Docu COMNT 2810downafter3rdPTLC

OBNUC $1 \mathrm{H}$

EXMOD single_pulse.ex2

$\quad 600.17 \mathrm{MHz}$

OBSET $\quad 5.30 \mathrm{KHz}$ OBFIN $\quad 5.47 \mathrm{~Hz}$ FREQU $\quad 14076.79 \mathrm{~Hz}$ $\begin{array}{ll}\text { SCANS } & 8 \\ \text { ACQTM } & 1.4549 \mathrm{sec}\end{array}$ $1.4549 \mathrm{sec}$
$\mathrm{PD}$$\quad 4.0000 \mathrm{sec}$ PW1 6.75 usec CTEMP $21.3 \mathrm{c}$ $\begin{array}{lrl}\text { SLVNT } & \text { CDCL3 } 3000 \\ \text { EXREF } & 0.00 \mathrm{pPm}\end{array}$ $\begin{array}{ll}\text { EXREF } & 0.00 \mathrm{ppm} \\ \text { BF } & 1.20 \mathrm{~Hz}\end{array}$

$1 \mathrm{H}-\mathrm{NMR}(\mathrm{CDC} / 3) \delta$ : $7.85(2 \mathrm{H}, d, J=8.9 \mathrm{~Hz})$, $7.73(2 \mathrm{H}, d, J=8.2 \mathrm{~Hz})$.
$7.30(5 \mathrm{H}, \mathrm{d} J=8.2 \mathrm{~Hz})$ $7.27\left(5 \mathrm{H}^{\prime}, \mathrm{d} J=8.2 \mathrm{~Hz}\right)$, $4.43(1 \mathrm{H}, d, J=9.6 \mathrm{~Hz})$, $3.85-3.80(1 \mathrm{H}, \mathrm{m})$.

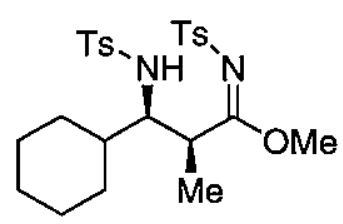

syn-7s 
C:\#Documents and Settings*All Users:Documents¥Matsubara\#2801-2900\#2810downafter3rdPTLC_CARBON.als

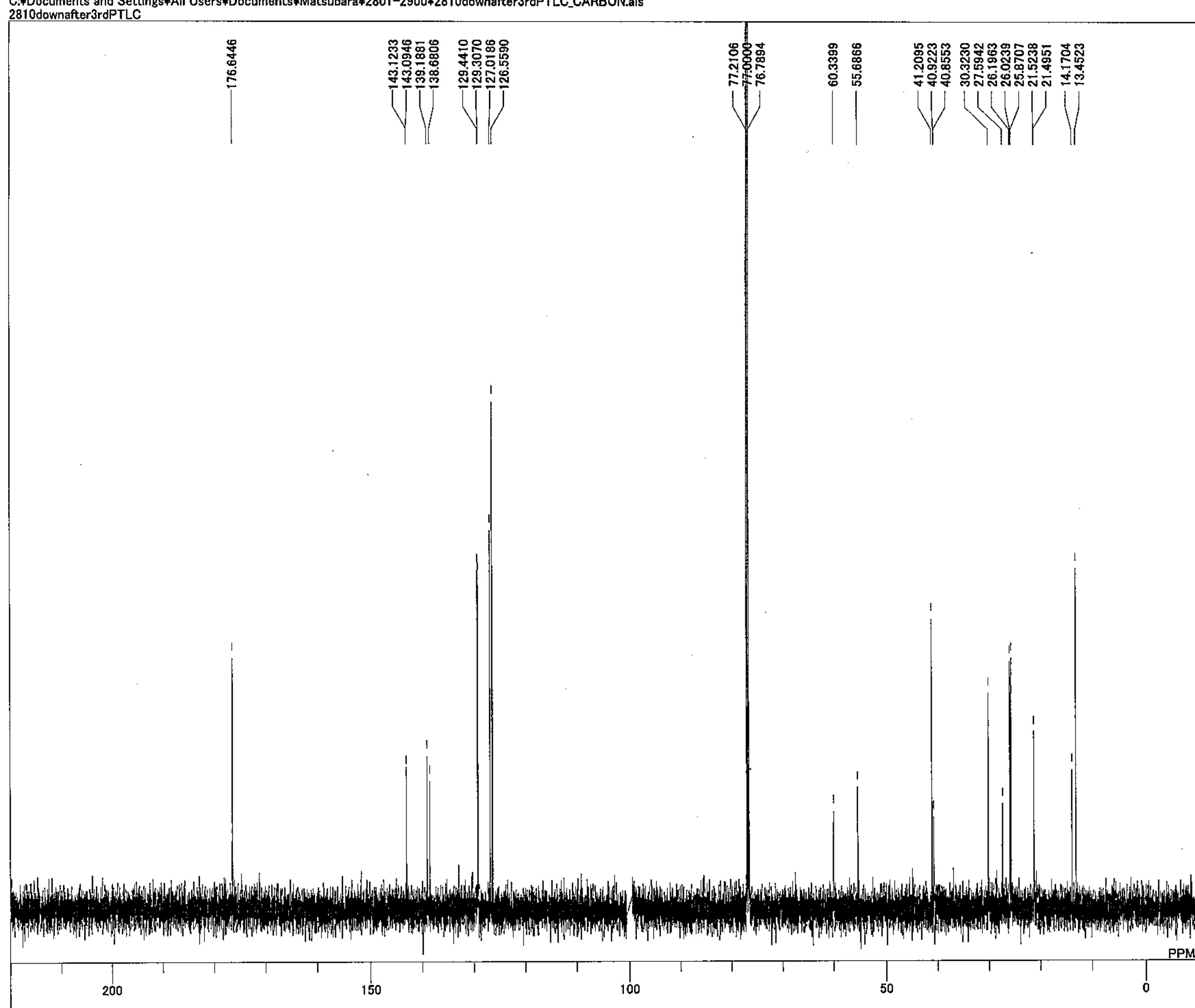

DFILE C:ҰDocuments and Settings $¥ A$ All Users*Docu

2810downafter3rdPT

OBNUC $13 \mathrm{C}$

EXMOD single_pulse_dec

OBFRQ $150.92 \mathrm{MHz}$

$\quad 8.52 \mathrm{KHz}$

POINT $1.74 \mathrm{HZ}$

FREQU $3787821 \mathrm{~Hz}$

SCANS $\quad 512$

ACQTM $\quad 0.6921 \mathrm{sec}$

PW1 $2.0000 \mathrm{sec}$

IRNUC $1 \mathrm{H}$

H.83 usec

CTEMP $\operatorname{CDCL}^{22.6 \mathrm{c}}$

$\begin{array}{ll}\text { EXREF } & 77.00 \mathrm{ppm} \\ \text { BF } & 0.12 \mathrm{~Hz}\end{array}$

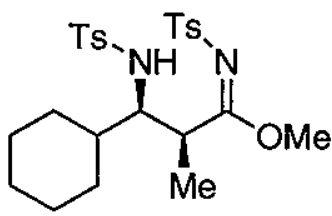

syn-7s 
C:FDocuments and Settings¥delta*My Documents*Personal Folder¥Matsubara*2801-2900*2830up_C.als

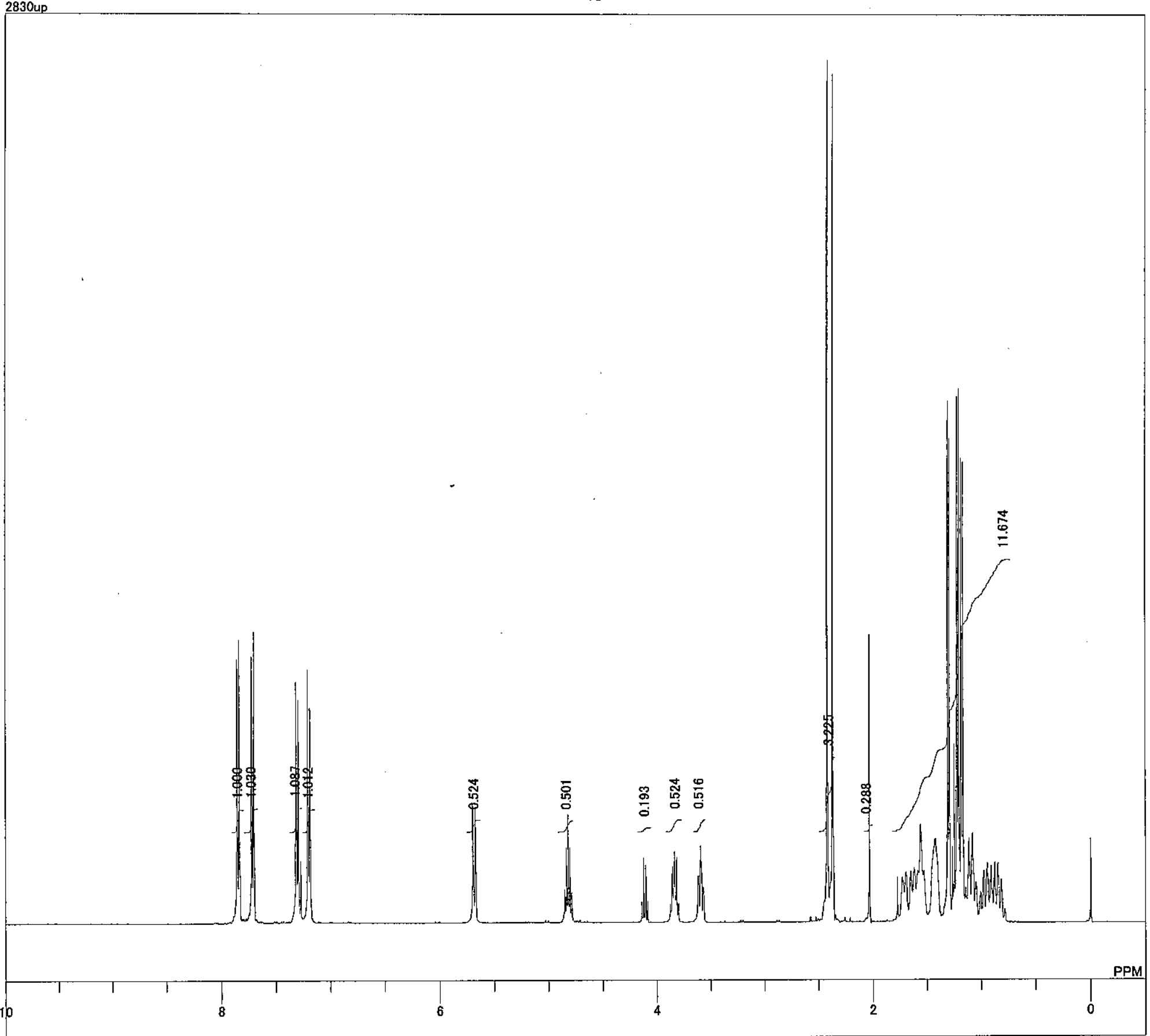

DFILE

COMNT

DATIM

OBNUC

OBFRQ

BSET

OBFIN

SCANS

ACQTM

PD1

IRNUC

CTEMP
SLNNT
EXREF
BFF

RF

ocuments and Settings $¥$ delta $¥$ My Documents $¥$ Person 14-12-2007 19:05:05

ingle.pulse.ex2 $4.19 \mathrm{KHz}$ $7.29 \mathrm{~Hz}$

$7503.00 \mathrm{~Hz}$

8

$2.1837 \mathrm{sec}$

$2.0000 \mathrm{sec}$
5.90 usec

$25.0 \mathrm{c}$

$0.00 \mathrm{ppm}$
$0.12 \mathrm{~Hz}$

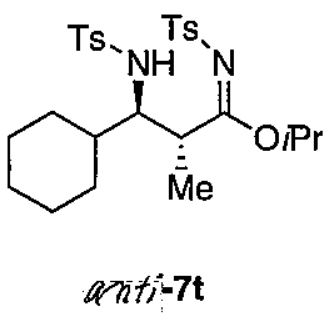




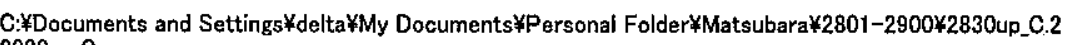

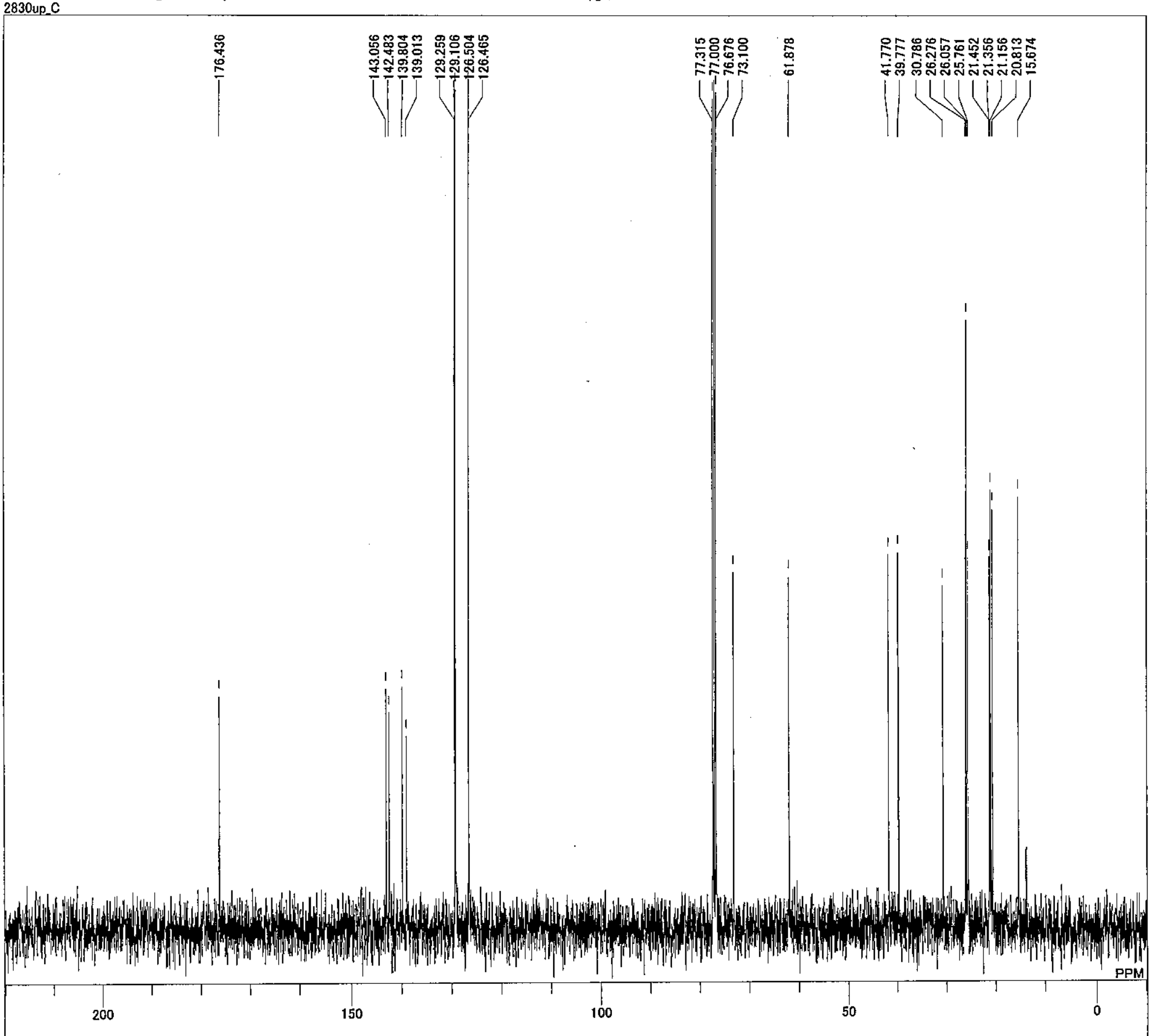

C:*Documents and Settings¥delta¥My DocumentsłPersor

$\begin{array}{rr}\text { EXMOD } & \text { single.pulse dec } \\ \text { OBFRQ } & 100.53 \mathrm{MHz}\end{array}$

OBSET $\quad 5.35 \mathrm{KHz}$

OBFIN $\quad 5.86 \mathrm{~Hz}$

FREQU $\quad 39259.39 \mathrm{~Hz}$

ACQTM $\quad 1.0433 \mathrm{se}$

$\begin{array}{lr}P D & 2.0000 \mathrm{sec} \\ \mathrm{PW} 1 & 2.83 \mathrm{usec}\end{array}$

IRNUC

CTEMP $25.0 \mathrm{c}$

EXREF $\quad 77.00 \mathrm{ppm}$

$\begin{array}{ll}\text { BF } & 0.12 \mathrm{~Hz} \\ \text { RGAIN } & 56\end{array}$

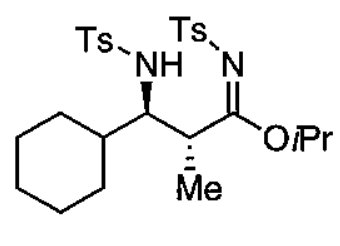

anti-7t 


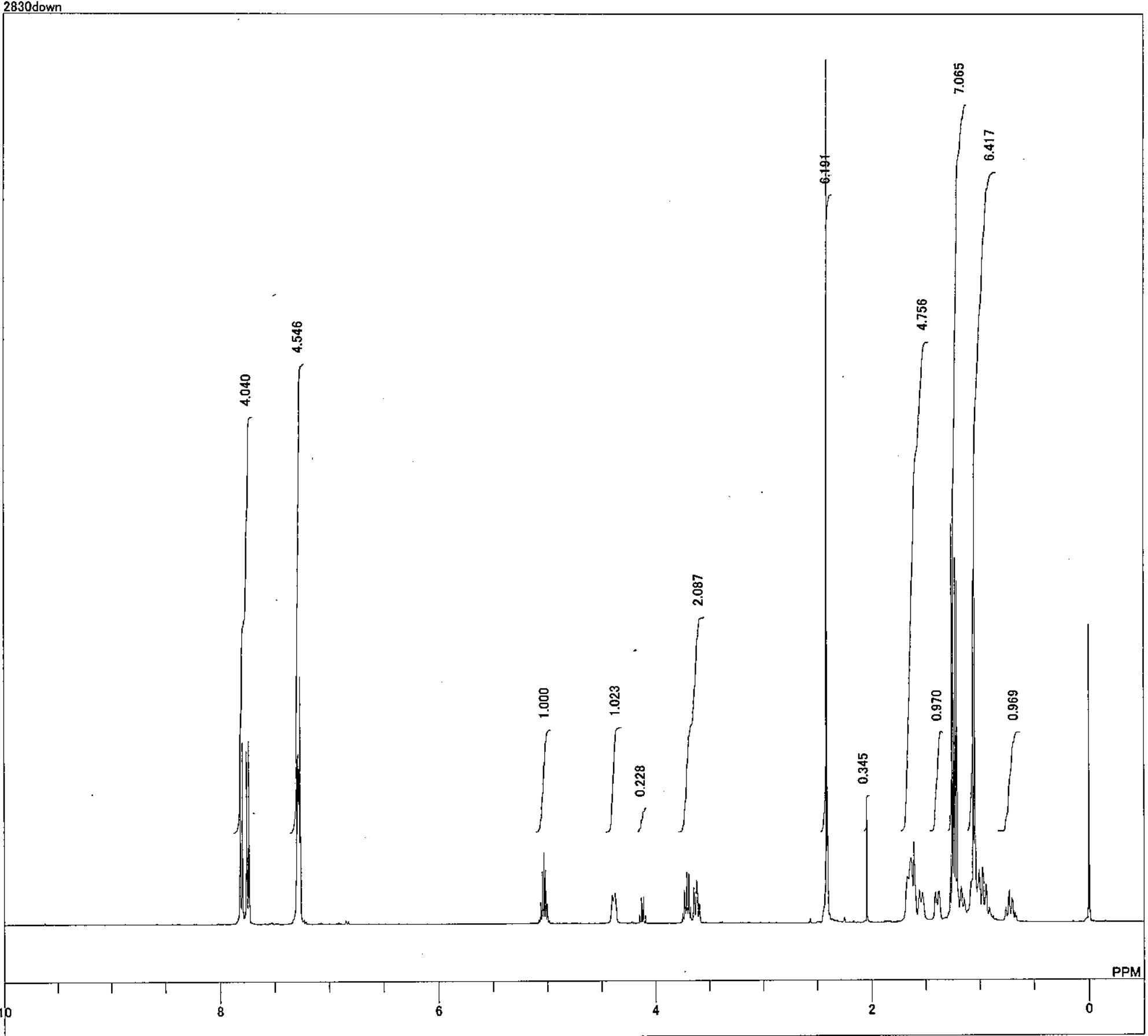

DFILE $\quad$ : $D$ Documents and Settings $¥ d$ delta\#My Documents $¥$ Person

DATIM

single.pulse.ex2

OBSET $\quad 4.19 \mathrm{KHz}$

OBFIN $\quad 7.29 \mathrm{~Hz}$

$600231 \mathrm{~Hz}$

SCANS $\quad 000.31 \mathrm{~Hz}$

ACQTM $2.1837 \mathrm{sec}$

$2.0000 \mathrm{sec}$

$\begin{array}{ll}2.90 & \text { usec }\end{array}$

CTEMP $25.1 \mathrm{c}$

SLVNT $\mathrm{CDCL}^{2} 3$

$\begin{array}{ll}\text { EXREF } & 0.00 \mathrm{ppm} \\ \text { BF } & 0.12 \mathrm{~Hz} \\ \text { RGAIN } & 34\end{array}$

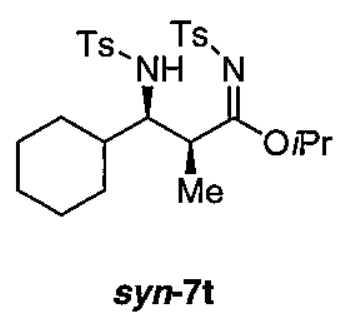




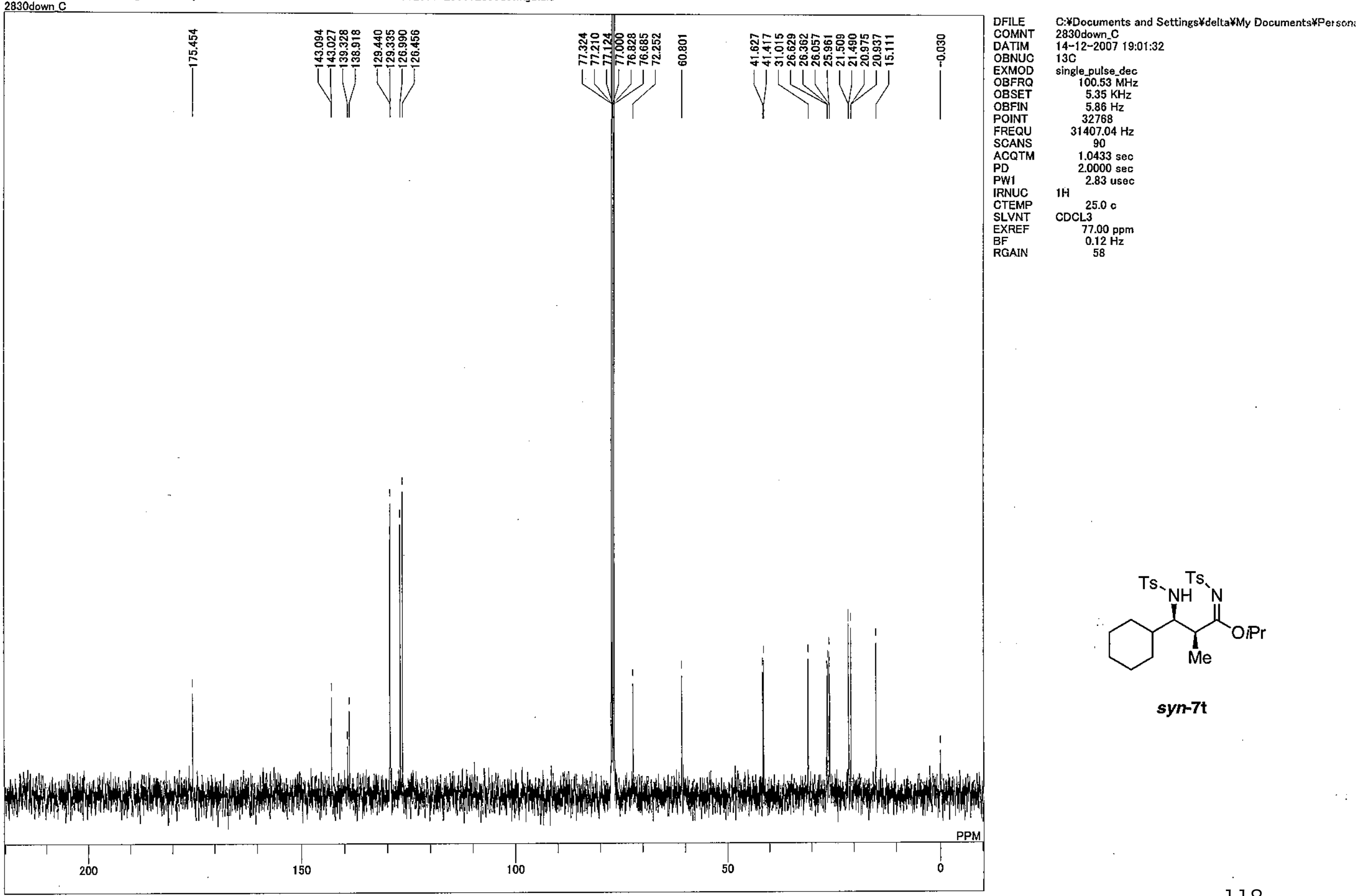


FREQU

SCANS
ACQTM

PD

PWI
IRNUC
CTEMP

CTEMP
SLVNT

SLVNT

EXREF

H NMR.ex2

$495.13 \mathrm{MHz}$

$4.38 \mathrm{KHz}$

$9.64 \mathrm{~Hz}$
13107

$7429.31 \mathrm{~Hz}$

8

6.50 usec

$22.3 \mathrm{o}$

$0.00 \mathrm{ppm}$

$0.00 \mathrm{ppm}$
$0.12 \mathrm{~Hz}$

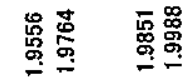
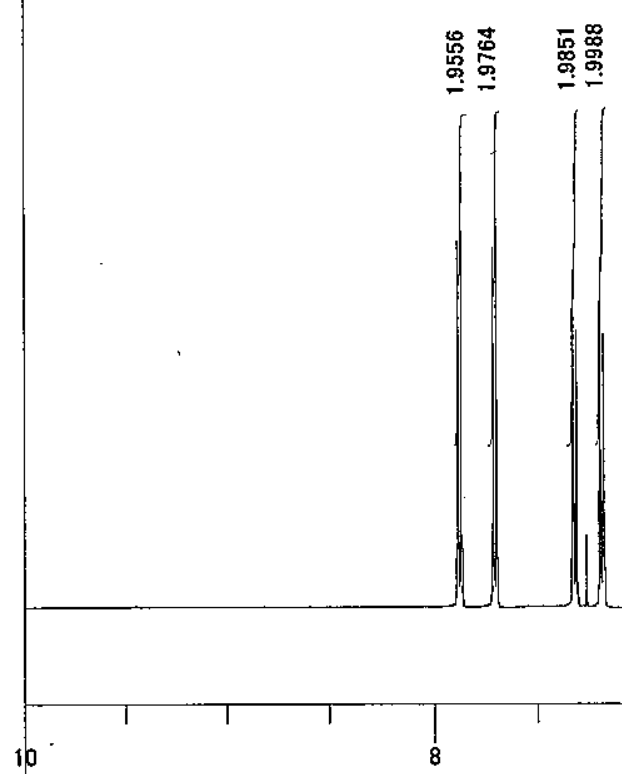

:

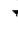
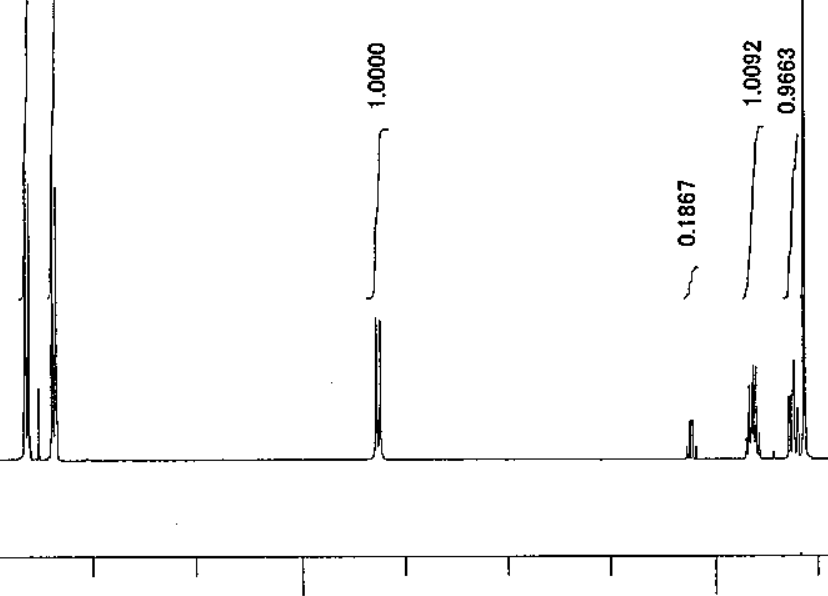

6

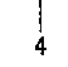

4

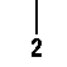

2 
C:\#Documents and Settings $¥ A l l$ Users $¥$ Documents $¥ M a t s u b a r a ¥ 2801-2900 * 2828$ middle_C_CARBON.3

2828middle_C
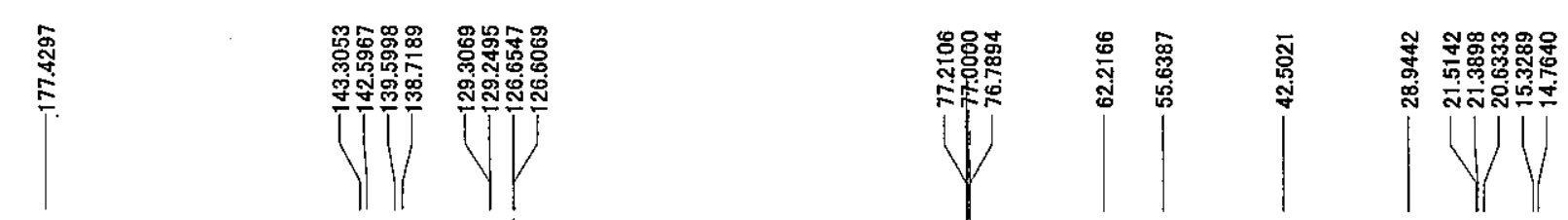

DFILE

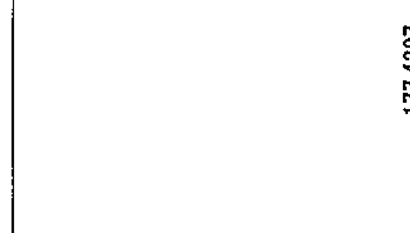

DATIM

OBFRQ

OBSET

OBFIN

FREQU

SCANS

ACQTM

PD 1 1

IRNUC

CTEMP

SLVNT

EXREF
BF
RGIN

$\operatorname{CDCL}^{22.0 \mathrm{c}}$

$77.00 \mathrm{ppm}$
$0.12 \mathrm{~Hz}$
58

-12-2007 19:25:31

ingle pulse dec
$150.92 \mathrm{MHz}$ $8.52 \mathrm{KHz}$ ${ }_{40961}^{1.74 \mathrm{~Hz}}$ $59186.51 \mathrm{~Hz}$ 256 $0.6921 \mathrm{sec}$ $2.0000 \mathrm{sec}$ 83 usec

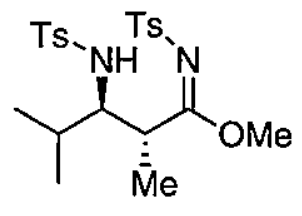

anti-7u 
C:\#Documents and Settings¥delta\#My Documents¥Personal Folder¥Matsubara¥2801-2900¥2828downafter2ndPTLC.als

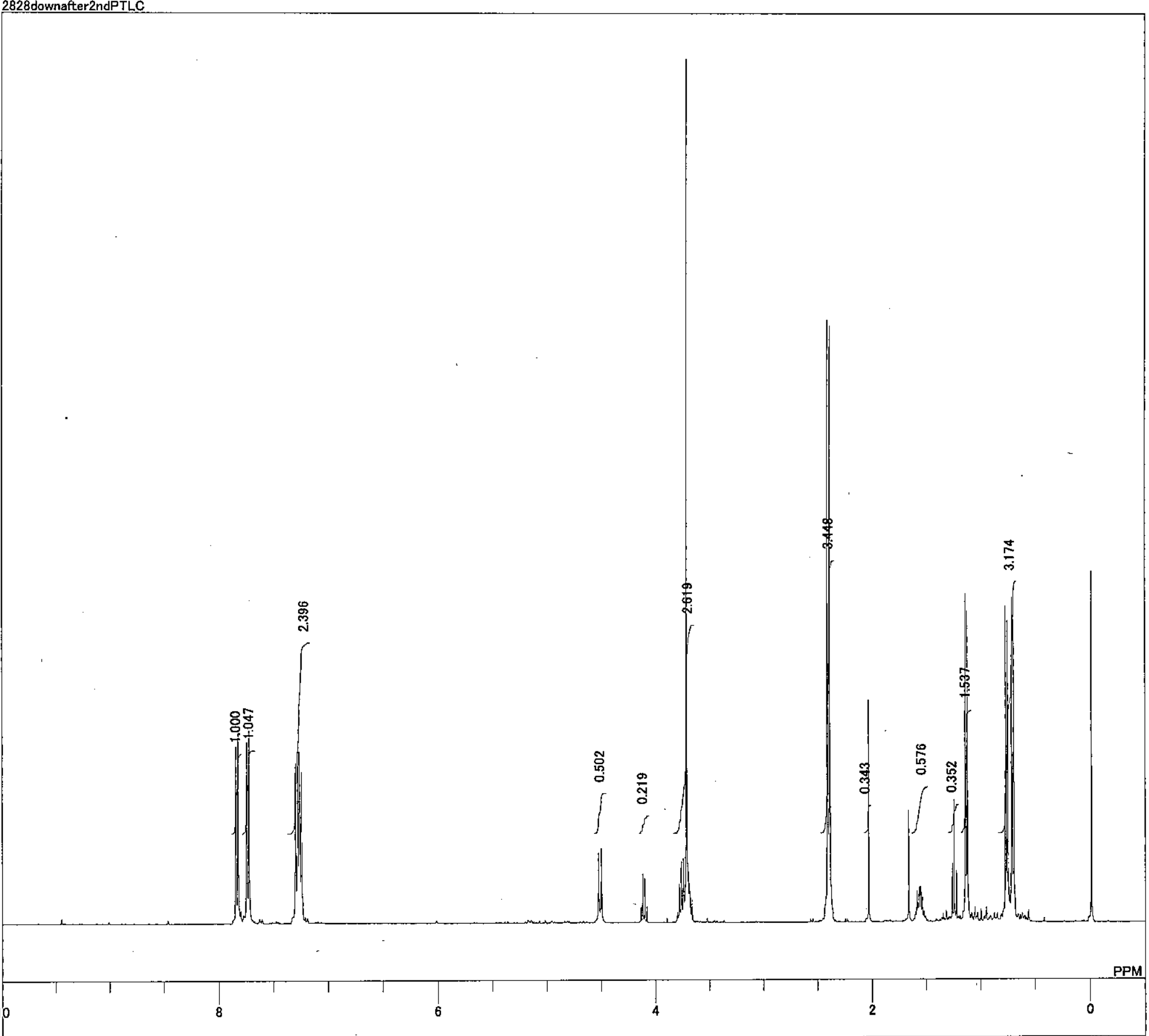

C:*Documents and Settings $¥$ delta*My Documents $¥ p_{\text {erso: }}$

COMNT

DATM

EXMOD

OBFRQ

OBFIN

FEQU

SCANS

ACQTM

D

PWI

CTEMP

SLVNT

EXREF

RGAIN

28downafter2ndPTLC

$399.78 \mathrm{MHz}$

$4.19 \mathrm{KHz}$

$7.29 \mathrm{~Hz}$

$7503.00 \mathrm{~Hz}$

$7503.00 \mathrm{~Hz}$

$2.1837 \mathrm{sec}$

$2.0000 \mathrm{sec}$

H 5.00 usec

$\operatorname{CDCL}^{25.1}$

$12.51 \mathrm{ppm}$
$0.12 \mathrm{~Hz}$
32
Ts ${ }_{N H}$ S $_{\text {N }}$

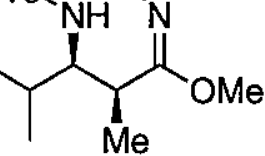

syn-7u 
C:FDocuments and Settings $¥ A \mid l$ Users¥Documents $¥$ Matsubara $¥ 2801-2900 * 2828$ down_C_CARBON.als

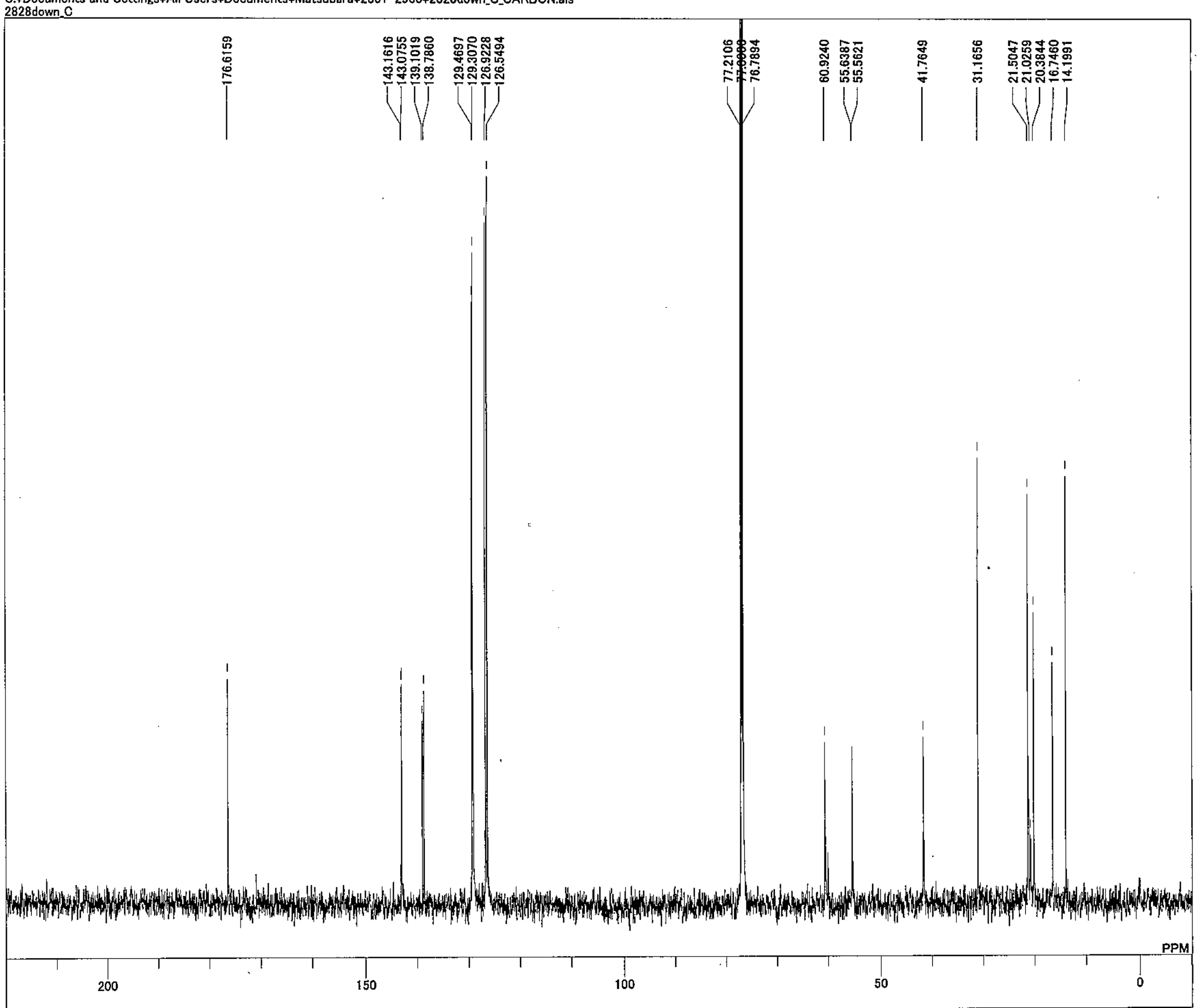

DFILE C:*Documents and Settings:A.ll Users¥Docu

$\begin{array}{ll}\text { COMNT } & \text { 2828down C } \\ \text { DATIM } & 13-12-2007 \text { 19:55:48 }\end{array}$

OBNUC 13C

EXMOD single_pulse_dec

$\begin{array}{lc}\text { OBFRQ } & 150.92 \mathrm{MHz} \\ \text { OBSET } & 8.52 \mathrm{KHz}\end{array}$

OBFIN $\quad 1.74 \mathrm{~Hz}$

POINT $\quad 32768$

FREQU $\quad 47348.48 \mathrm{~Hz}$

$\begin{array}{ll}\text { SCANS } & 400 \\ \text { ACQTM } & 0.6921 \mathrm{sec}\end{array}$

$2.0000 \mathrm{sec}$

2.83 useo

CTEMP $21.7 \mathrm{c}$

SLVNT CDCL3. $77.00 \mathrm{pom}$

$\begin{array}{ll}\text { BF } & 77.00 \mathrm{ppm} \\ \text { RGAIN } & 60 \mathrm{~Hz}\end{array}$

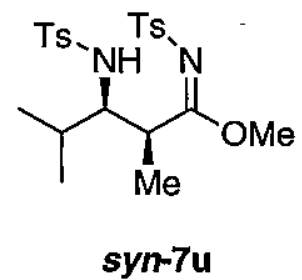

syn-7u 


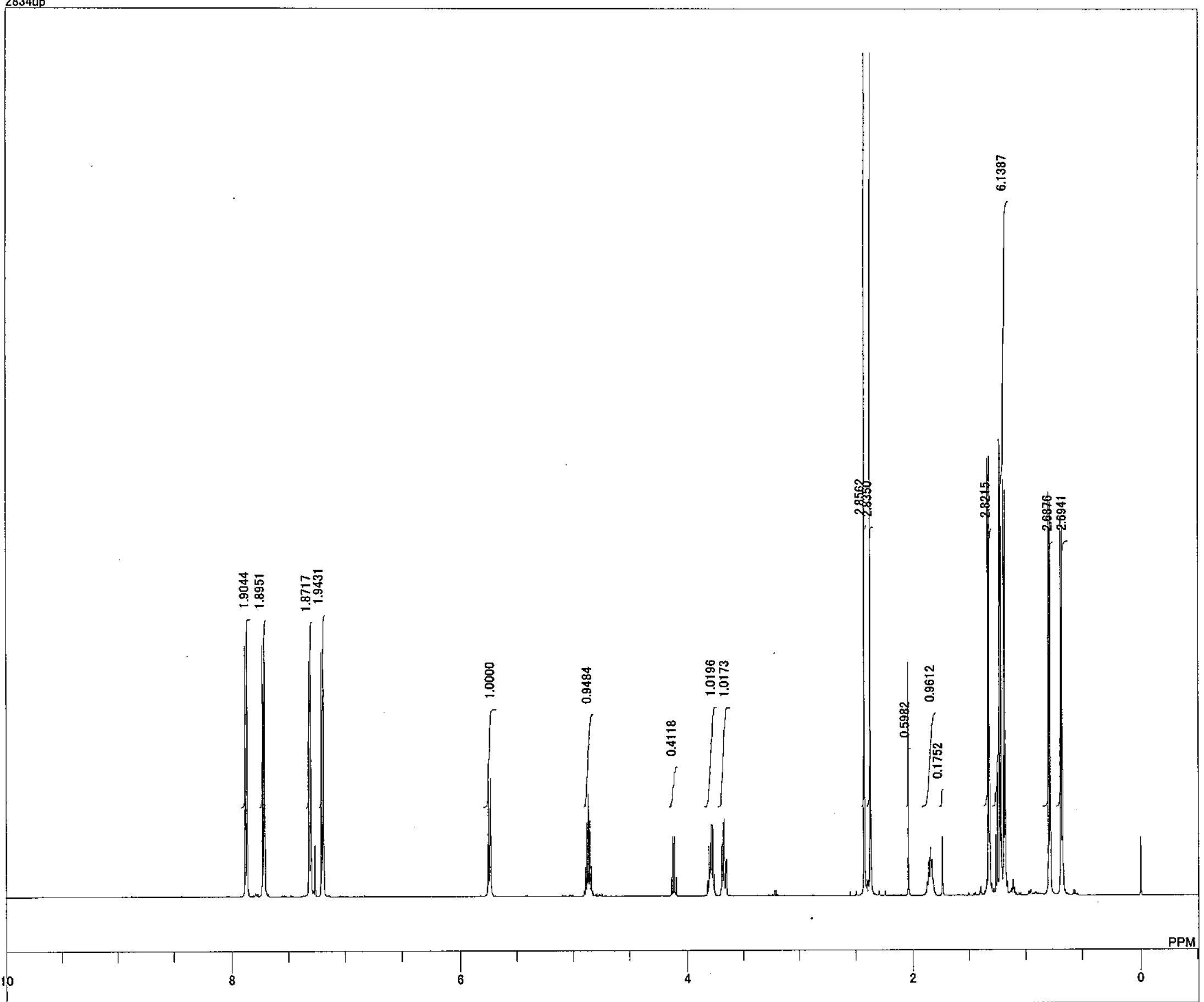

DFILE C:*Documents and Settings $¥ A$ All Users $¥$ Docu up

EXMOD

OBFRO

OBSET

N95.13 $\mathrm{MHz}$

$\quad 4.38 \mathrm{KHz}$

$7427.21 \mathrm{~Hz}$

SCANS

ACQTM

PW1

$5.0000 \mathrm{sec}$
$6.50 \mathrm{usec}$

CTEMP $21.7 \mathrm{c}$

EXREF $\quad 0.00 \mathrm{ppm}$

RGAIN

$0.00 \mathrm{ppm}$
$0.12 \mathrm{~Hz}$
34

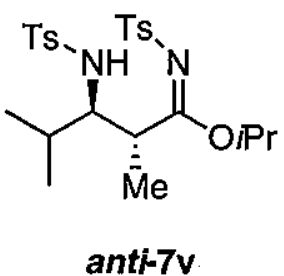




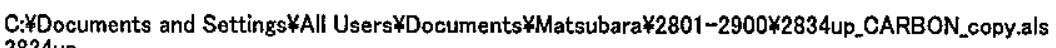

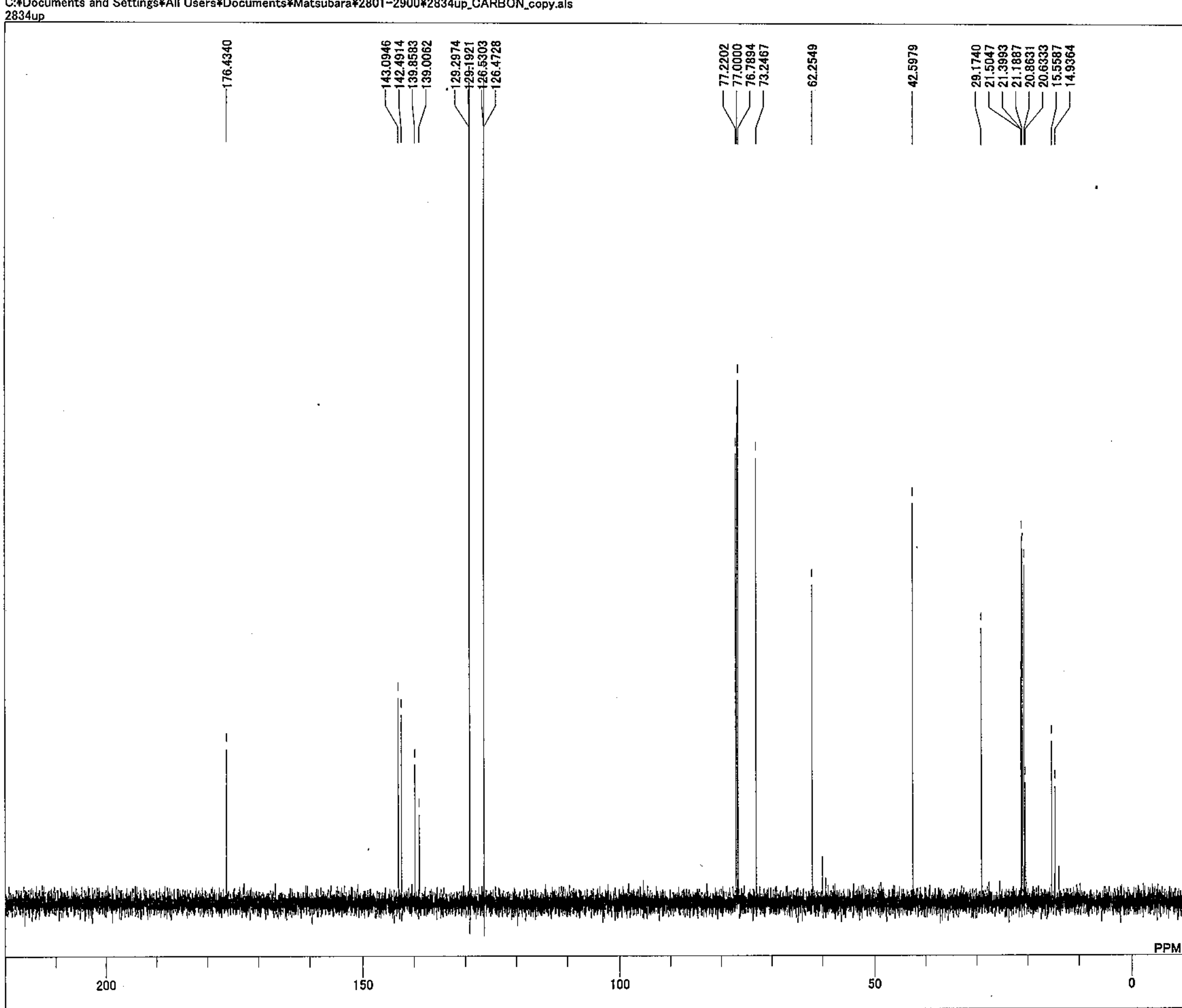

DFILE C:*Documents and Settings*All Users*Docu

DATIM 2834up

OBFRO

OBSET

OBFIN

FREQU

ACQTM

PW1

IRNUC

SLVNT

BF

ingle_pulse_dec

$150.92 \mathrm{MHz}$ $8.52 \mathrm{KHz}$ 1.714 $37878.21 \mathrm{~Hz}$ 44 $0.6921 \mathrm{sec}$ $2.0000 \mathrm{sec}$ $1 \mathrm{H} \quad 2.83$ useo CDCL $3.0 \mathrm{C}$ $77.00 \mathrm{ppm}$
$0.12 \mathrm{~Hz}$

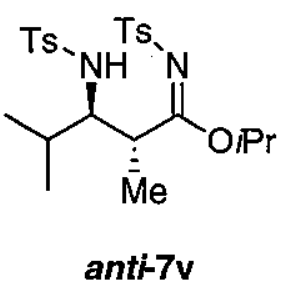

anti-7v 
C:FDocuments and Settings $\# A l l$ Users*Documents $\#$ Matsubara $¥ 2801-2900 * 2834$ downafter2ndPTLC-1.als
2834downafter2ndPTLC.

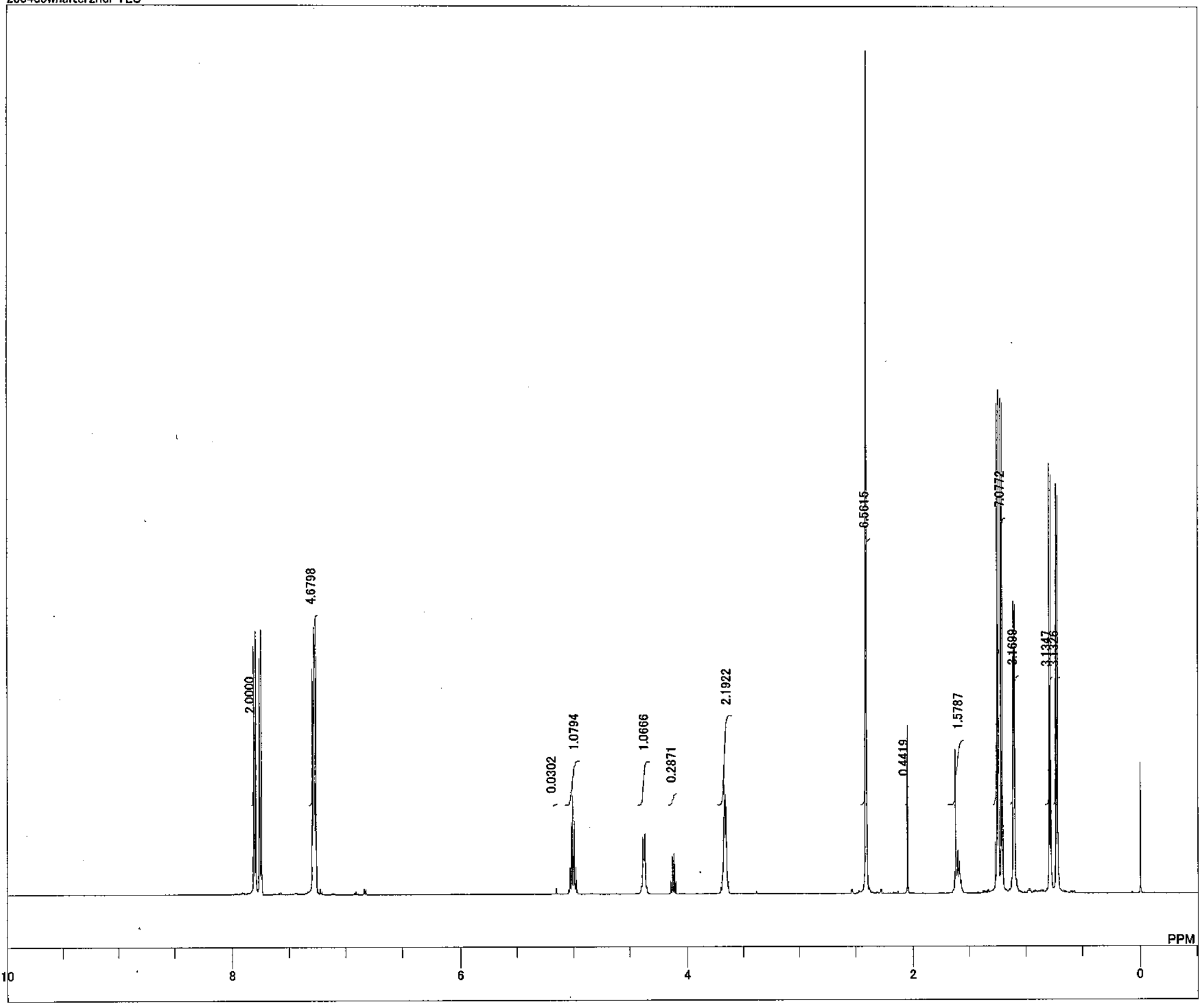

PM
OBFRO

OBSET

OBFIN

FREQU

SCANS

ACOTM

PD

IRNUC

CTEMP

SLVNT

EXREF

RGAIN

:WDocuments and Settings:All Users*Docu 12-2007 13:50:53

NMR.ex 2

$495.13 \mathrm{MHz}$ $4.38 \mathrm{KHz}$ $9.64 \mathrm{~Hz}$ $7429.31 \mathrm{~Hz}$

8

$1.7642 \mathrm{sec}$ 6.50 usec $21.6 \mathrm{c}$ $0.00 \mathrm{ppm}$ $0.12 \mathrm{~Hz}$

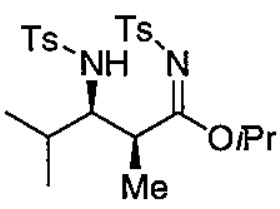

$5 y n-7 v$ 
C:FDocuments and Settings¥All Users¥Documents¥Matsubara\#2801-2900\%2834downafter2ndPTLC_C-1.als

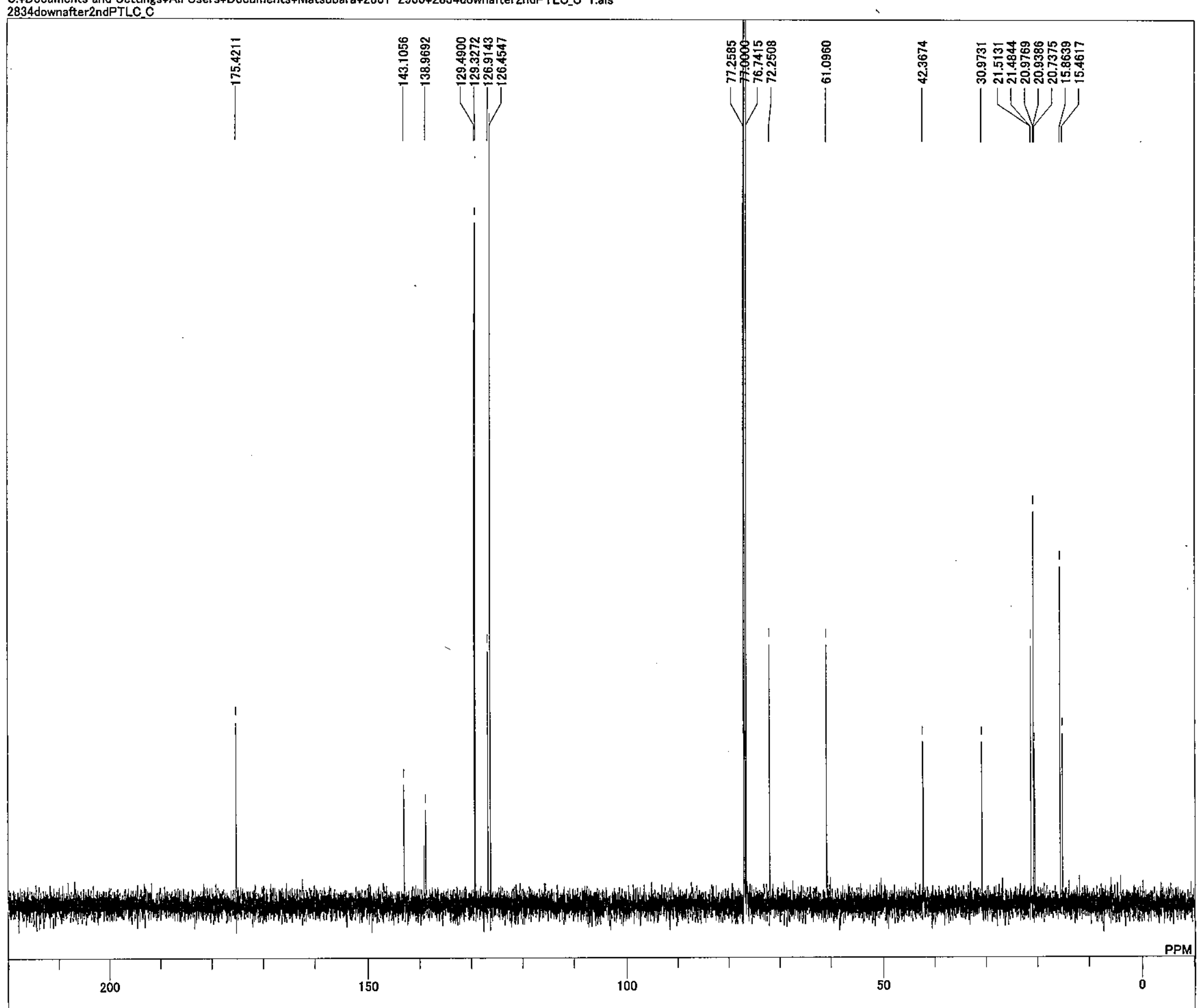

DFILE C:FDocuments and Settings*All Users*Docu

DATM

OBNUC

EXMOD

OBFRQ

OBSET

OBFIN
POINT

FREQU

SCANS

PD

PWI
IRNUC $1 \mathrm{H}$

CTEMP

SLVNT

EXREF
BF
RGAIN

C NMR.ex2

$124.51 \mathrm{MHz}$

$3.45 \mathrm{KHz}$ $6.00 \mathrm{~Hz}$
26214 $31249.52 \mathrm{~Hz}$

496 $0.8389 \mathrm{sec}$ $2.0000 \mathrm{sec}$ $22.2 \mathrm{c}$

$\mathrm{CDCL}^{2} .2 \mathrm{C}$
$77.00 \mathrm{ppm}$ $77.00 \mathrm{ppm}$
$0.12 \mathrm{~Hz}$
50

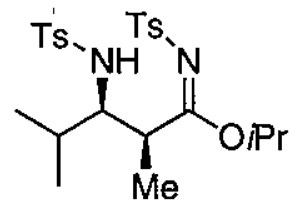

$5 y n-7 v$ 
EXMOD

OBFRQ

OBSET

OBFIN

PREQU

SCANS

ACQTM

PD

PW1

CTEMP

SLVNT

RGAIN

Documents and Settings¥All Users¥Docu 200product-up

single_pulse.ex2 $600.17 \mathrm{MHz}$ $5.30 \mathrm{KHz}$ 20480 $14076.79 \mathrm{~Hz}$ 16 $1.4549 \mathrm{sec}$ $4.0000 \mathrm{sec}$
$6.75 \mathrm{usec}$ 醇 $\operatorname{CDCL}^{21.1 \mathrm{c}}$ $0.00 \mathrm{ppm}$
$0.12 \mathrm{~Hz}$
40

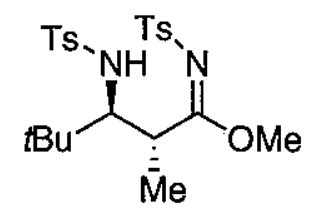

anti-7w

PPM 


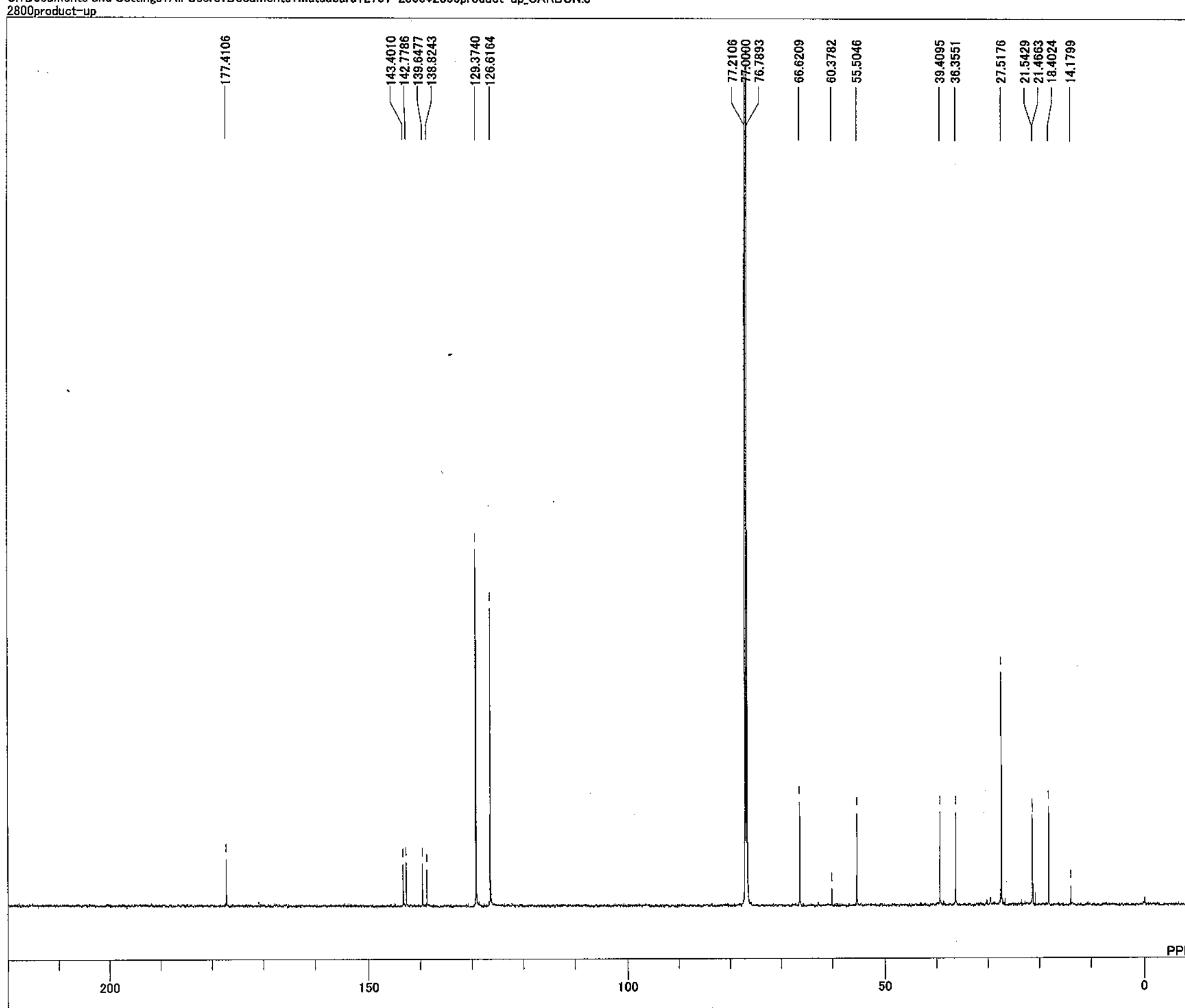

DFILE C:FDocuments and Settings $¥ A l l$ Users¥Docu

$\begin{array}{ll}\text { COMNT } & \text { 2800product-up } \\ \text { DATIM } & 30-11-200711: 12: 32\end{array}$

OBNUC 130

EXMOD single_pulse_dec

OBFRQ $\quad 50.92 \mathrm{MHz}$

OBSEN $\quad 8.52 \mathrm{KHz}$

POINT $\quad 40961$

FREQU $\quad 59186.51 \mathrm{~Hz}$

SCANS $\quad 4000$

ACQTM $\quad 0.6921 \mathrm{sec}$

PW1 $\quad 2.0000 \mathrm{sec}$

IRNUC $\quad 1 \mathrm{H}$

$22.0 \mathrm{c}$

SLVNT $\quad \mathrm{CDCL} 3$

$\begin{array}{lc}\text { EXREF } & 225.39 \mathrm{ppm} \\ \text { BF } & 0.12 \mathrm{~Hz} \\ \text { RGAIN } & 58\end{array}$

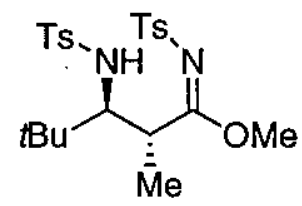

$a n+i-7 w$ 
OBFRQ

PBIN

POINT

SCANS

ACQTM

PD

PW1

CTEMP

SLVNT

$\underset{B E F F}{\text { EXREF }}$

BFAIN

WDocuments and Settings*All Users*Docu 0-11-2007 11:23:52

ingle_pulse.ex2 $600.17 \mathrm{MHz}$ $5.30 \mathrm{KHz}$ $5.47 \mathrm{~Hz}$ $14076.79 \mathrm{~Hz}$

16

$1.4549 \mathrm{sec}$ $4.0000 \mathrm{sec}$

$1 \mathrm{H} \quad 21.0 \mathrm{c}$

CDCL $21.0 \mathrm{c}$

$0.00 \mathrm{ppm}$
$0.12 \mathrm{~Hz}$
48

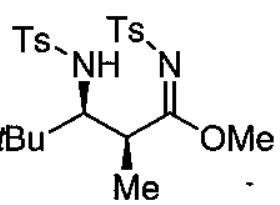

$5 y n-7 w$ 


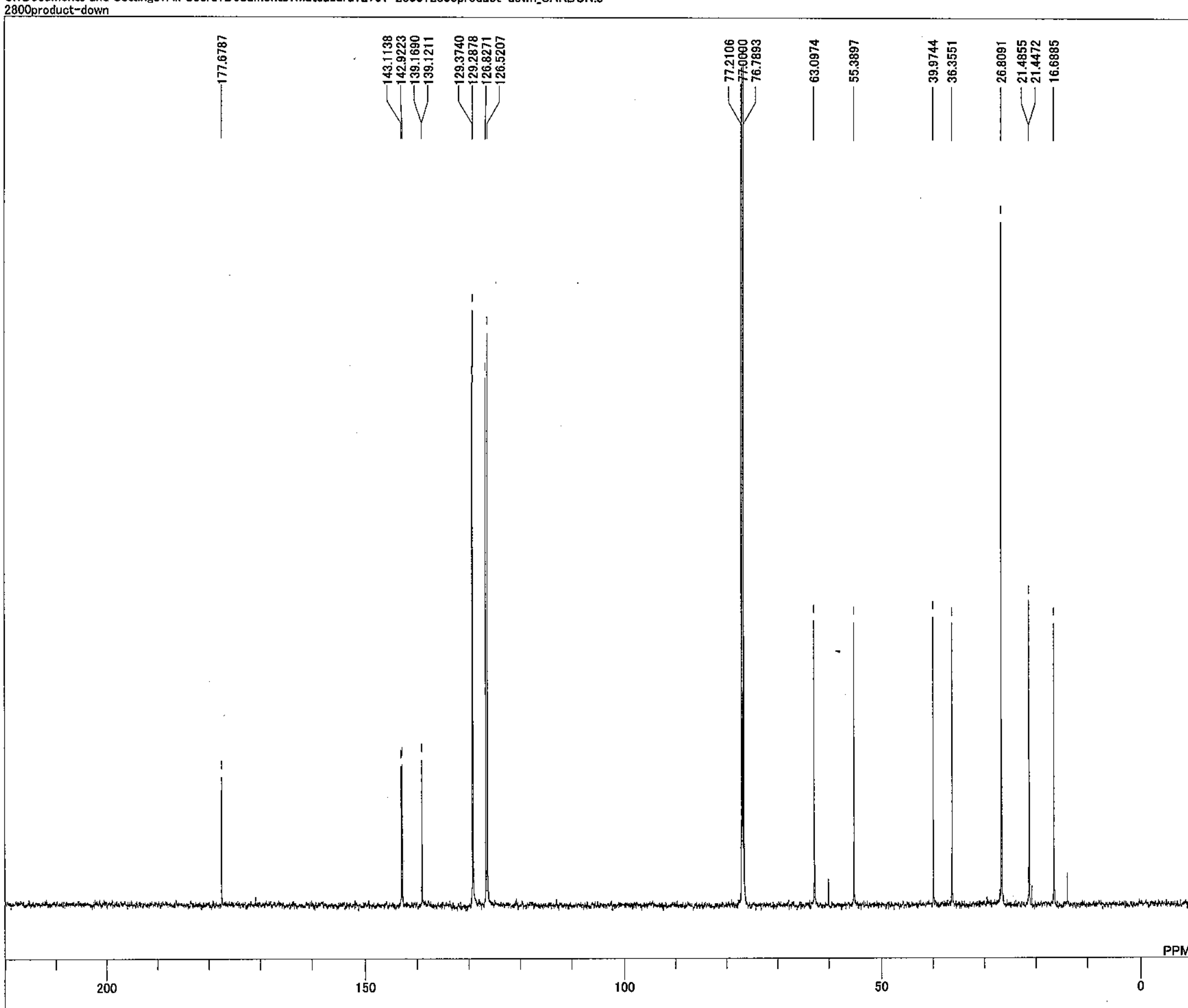

DFILE C: $:$ Documents and Settings $¥ A$ All Users $¥$ Docu DATIM 30-11-2007 12:12:40

EXMOD single,pulsedec

OBFRQ $\quad 0.92 \mathrm{MHz}$

OBFEN $\quad 8.52 \mathrm{KHz}$

POINT 40961

FREQU $\quad 59186.51 \mathrm{~Hz}$

SCANS $\quad 1024$

$\quad 0.6921 \mathrm{sec}$

PW1 $2.0000 \mathrm{sec}$

CTEMP 22.5

CDCL3

$\begin{array}{lc}\text { EXREF } & 225.36 \mathrm{ppm} \\ \text { BF } & 0.12 \mathrm{~Hz} \\ \text { RGAIN } & 58\end{array}$

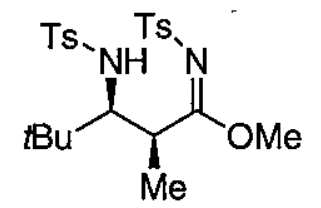

$\operatorname{syn}-7 w$ 


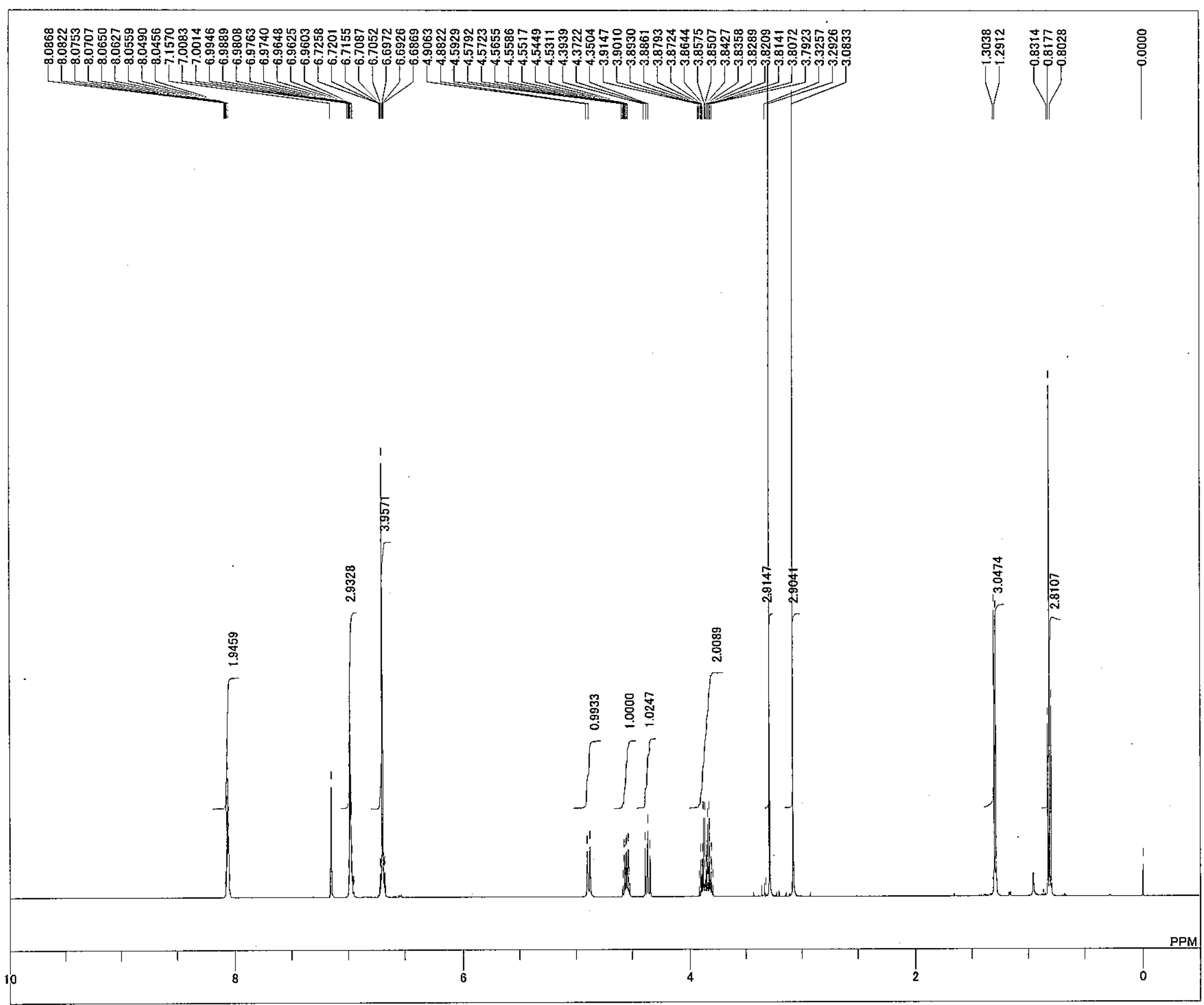

DFILE

COMN

DATIM

EXMOD

OBFRO

OBSET

OBIN

FREQU

SCANS

ACQTM

PCQT

IRNUC

CTEMP

SLVNT

EXREF

RGAIN

Dettings:Ail Users $¥$ Docu

5-07-2007 14:52:06

NMR.ex2

$495.13 \mathrm{MHz}$
$4.38 \mathrm{KHz}$

$9.64 \mathrm{~Hz}$

$7429.31 \mathrm{~Hz}$
8

$1.7642 \mathrm{sec}$
$5.0000 \mathrm{sec}$

$5.0000 \mathrm{sec}$
$6.50 \mathrm{usec}$

C6D ${ }^{25.1 \mathrm{c}}$

$0.00 \mathrm{ppm}$
$0.12 \mathrm{~Hz}$
30

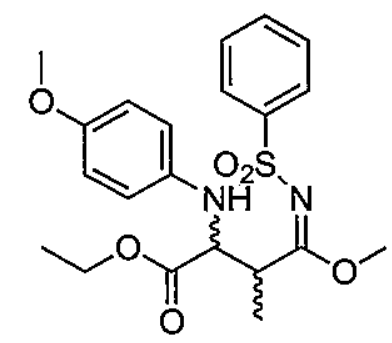

$7 \times$ (major) 


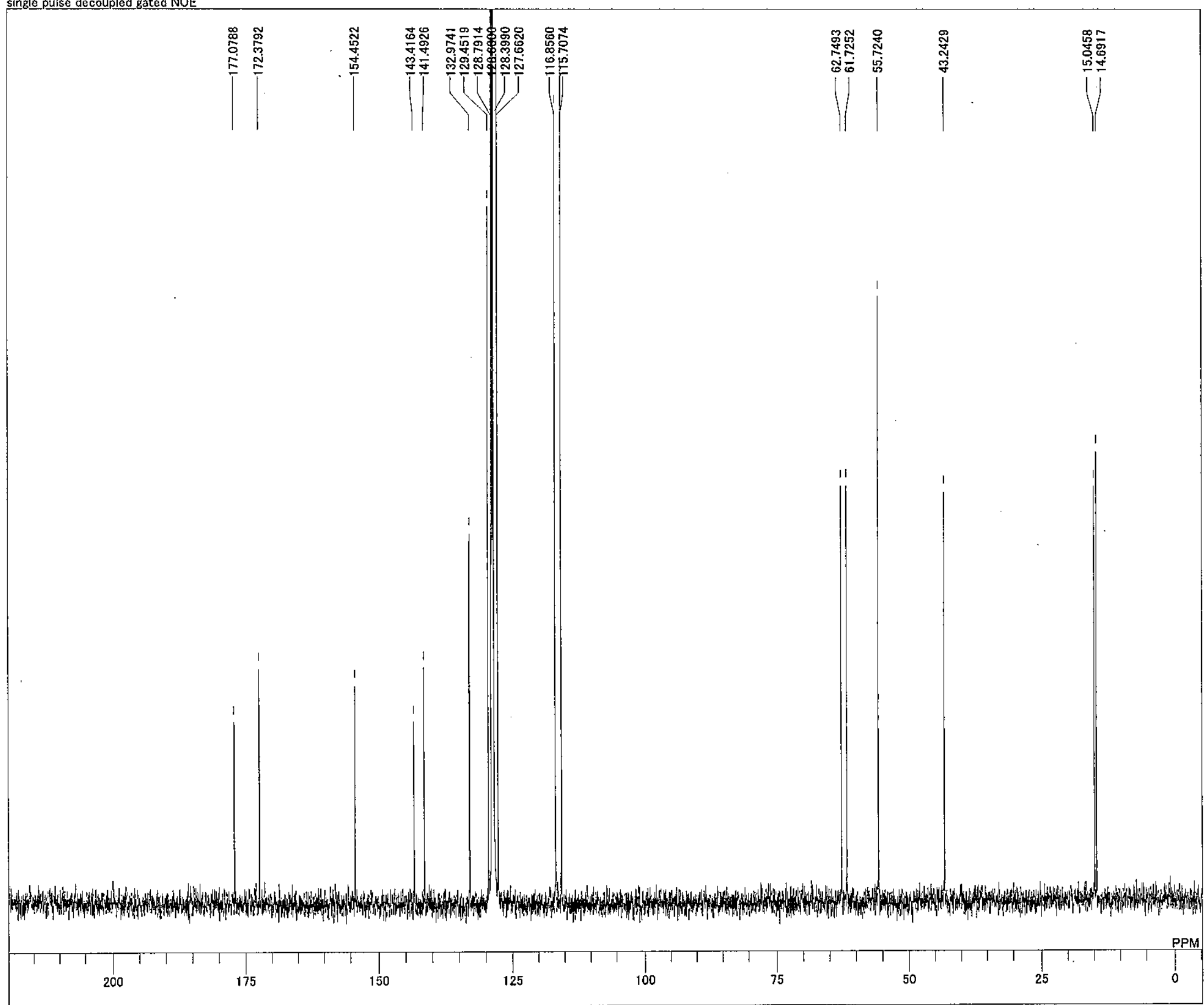

DFILE

DATIM

OBNUC

EXMOD

OBFRQ

OBFIN

POINT

FREQU

SCANS

PD

IRNUC

CTEMP

SLVNT

RGAIN
C: Documents and Settings $¥ A$ All Users $¥ D$ ocu Cingle pulse decoupled gated NO

C NMR.ex2 $124.51 \mathrm{MHz}$ $3.45 \mathrm{KHz}$ $6.00 \mathrm{~Hz}$
26224 $31249.52 \mathrm{~Hz}$ $0.8389 \mathrm{sec}$ $2.0000 \mathrm{sec}$
$3.67 \mathrm{usec}$

IH $25.7 \mathrm{c}$

C606 $128.60 \mathrm{ppm}$ $0.12 \mathrm{~Hz}$

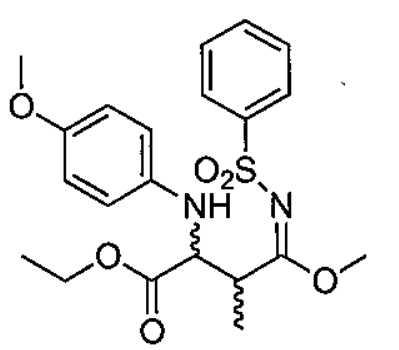

$7 \times($ major) 


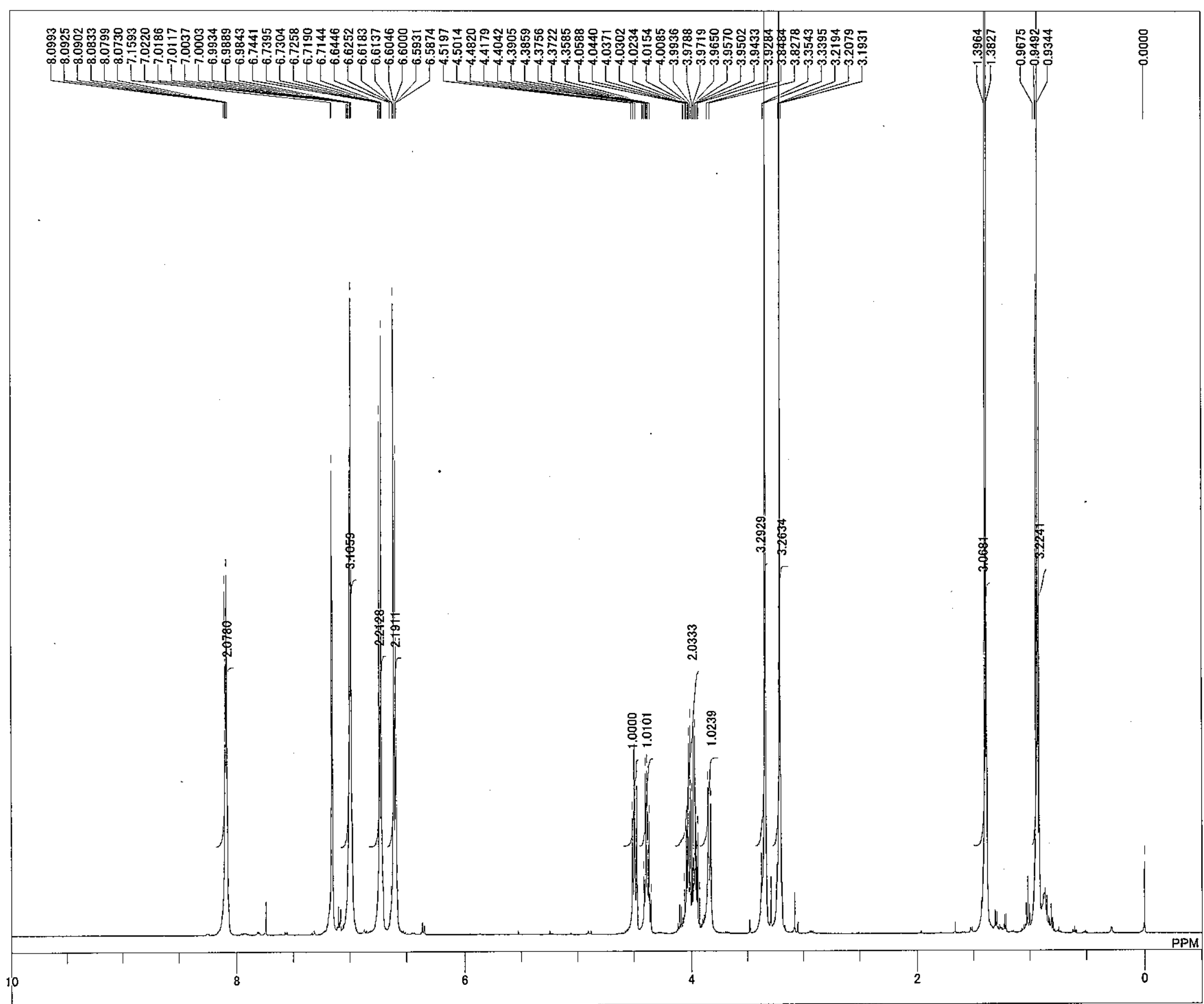

DFILE

C:FDocuments and Settings $¥ A l l$ Users $¥$ Docu

05-07-2007 14:57:53

OBNUC

OBFRQ

N95.ex2 2

$495.13 \mathrm{MHz}$
$4.38 \mathrm{KHz}$

$\quad 9.64 \mathrm{~Hz}$

13120
7429.31
$\mathrm{~Hz}$

SCANS

ACQTM

ACQTH

$1.7642 \mathrm{sec}$

$5.0000 \mathrm{sec}$

RNUC $1 \mathrm{H} 250$

V.NT 25.1

EXREF

$\mathrm{C} 606{ }_{0.00 \mathrm{ppm}}$

$0.00 \mathrm{ppm}$
$0.12 \mathrm{~Hz}$

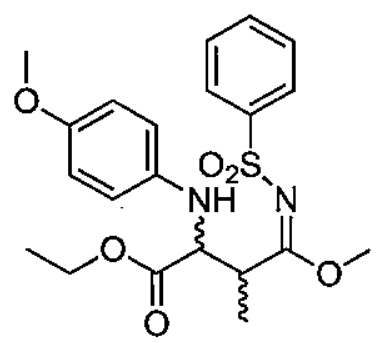

$7 \times$ (minor) 

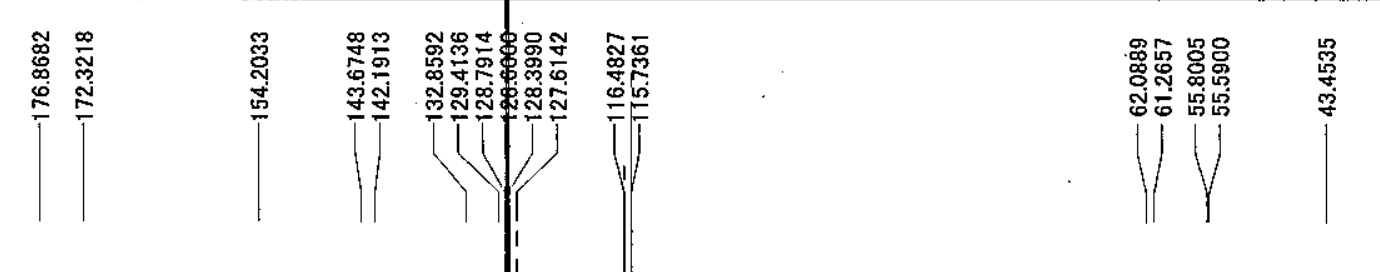

DFILE

OBNUC

OBFRQ

OBFIN
POINT
FREQU
SCANS

SCANS
ACOTM
PD

PD 1

IRNUC

CTEMP

SLVNT

RF

C:FDocuments and Settings \#All Users¥Docu single pulse decoupled gated NOE

$13 \mathrm{C}$ NMR.ex2

$124.51 \mathrm{MHz}$
$3.45 \mathrm{KHz}$

$6.00 \mathrm{~Hz}$

26224
$31249.52 \mathrm{~Hz}$

$31249.52 \mathrm{H}$
0.89

$0.8389 \mathrm{sec}$
$2.0000 \mathrm{sec}$

$1 \mathrm{H} \quad 3.67$ usec

${ }^{2} 66^{25.8 \mathrm{C}}$

$128.60 \mathrm{ppm}$
$0.12 \mathrm{~Hz}$
50

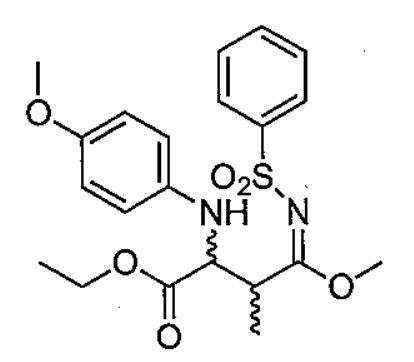

$7 \times$ (minor)

M

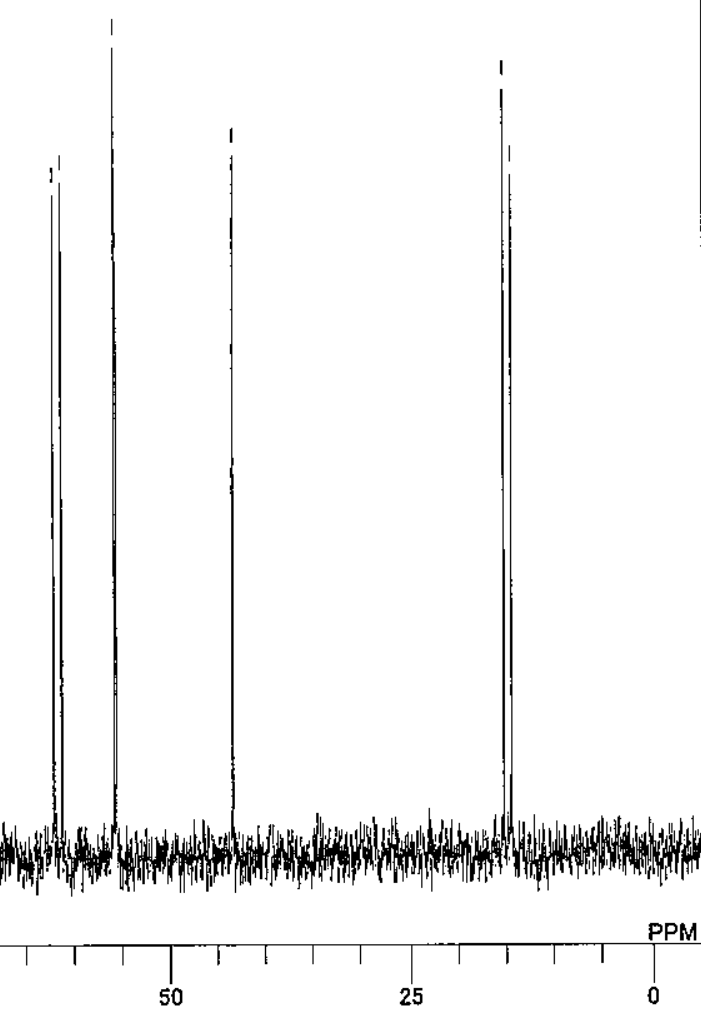

100

75 


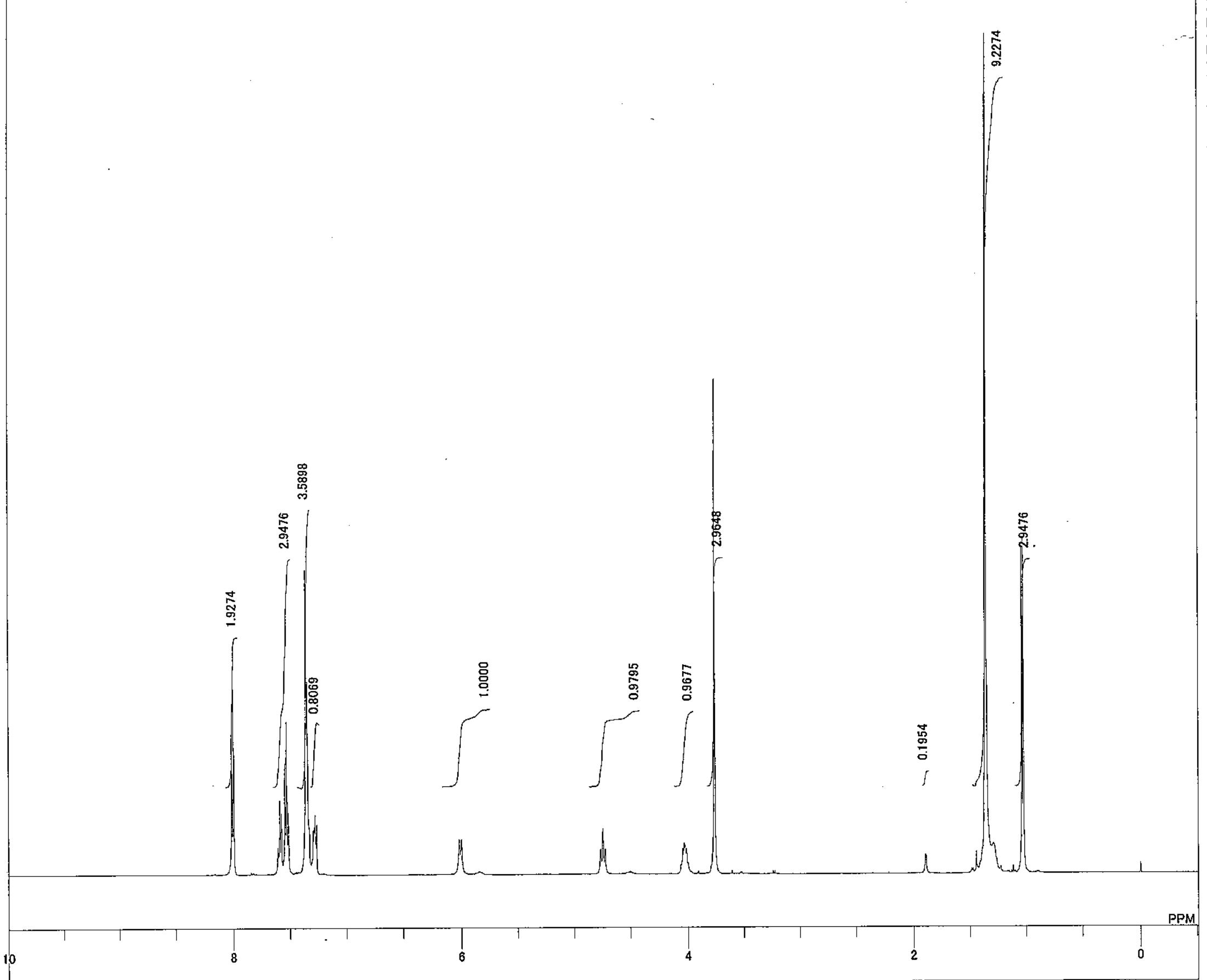


C:*Documents and Settings $¥ A$ All Users*Documents $¥$ Matsubara $¥ 2701-2800 * 2720$-data_C-1.als

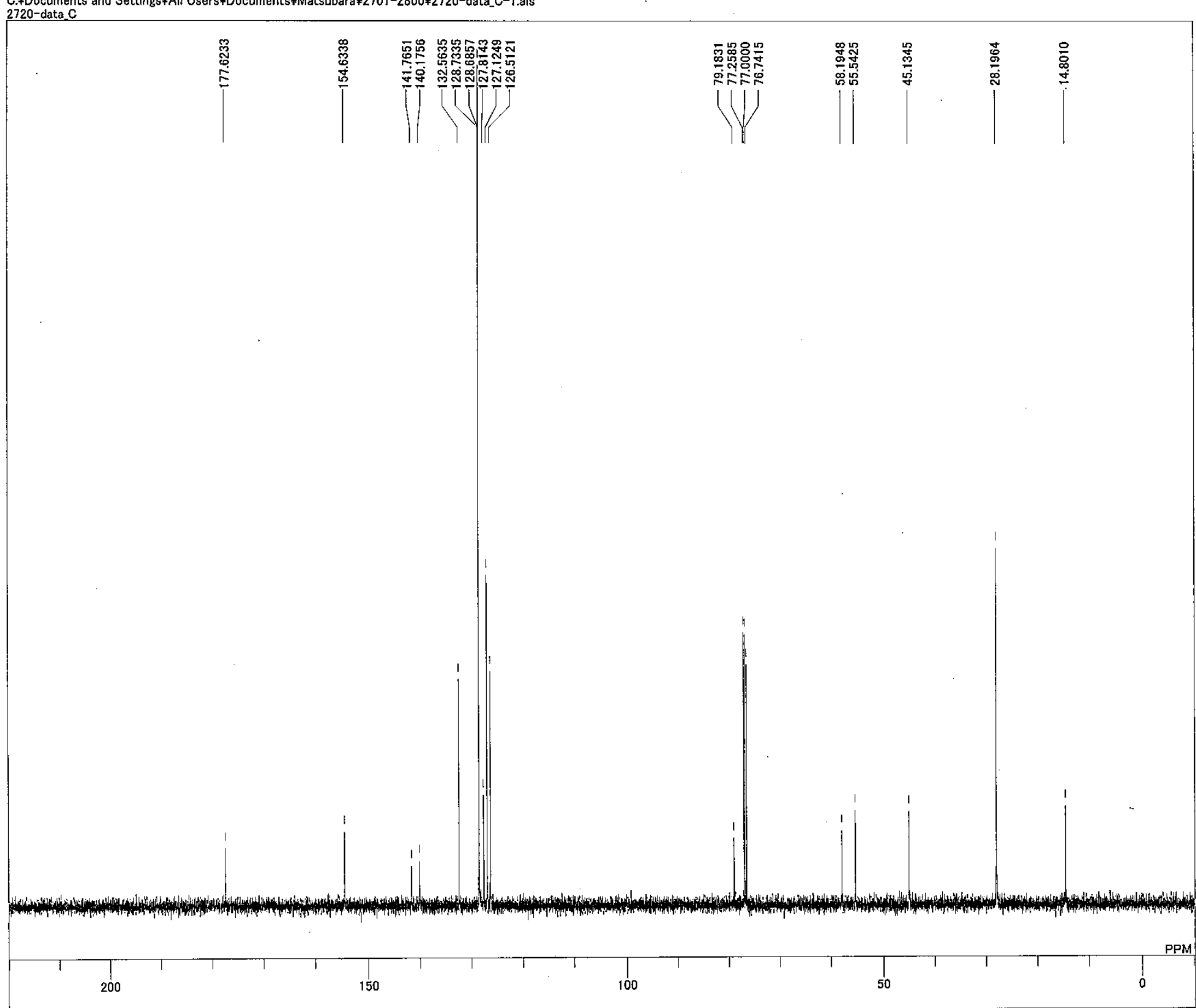

DFILE $\quad$ C: *Documents and Settings $¥ A l l$ Users $¥$ Docu

DATIM $\quad$ 2720-data C 11 -08-2007 13:55:18

EXMOD 13 C NMR.ex2

$\quad 124.51 \mathrm{MHz}$

OBSET $\quad 3.45 \mathrm{KHz}$

$\quad 6.00 \mathrm{~Hz}$

$\quad 31249.52 \mathrm{~Hz}$

$\begin{array}{lc}\text { SCANS } & 68 \\ \text { ACQTM } & 0.8389 \mathrm{sec}\end{array}$

$2.0000 \mathrm{sec}$

3.67 usec

CTEMP 29.9

$\begin{array}{lc}\text { EXREF } & 77.00 \mathrm{ppm} \\ \text { BF } & 0.12 \mathrm{~Hz} \\ \text { RGAIN } & 60\end{array}$

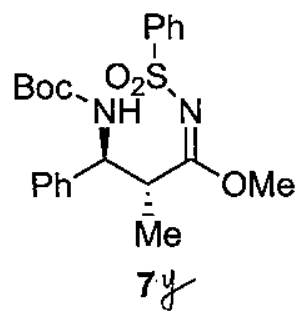




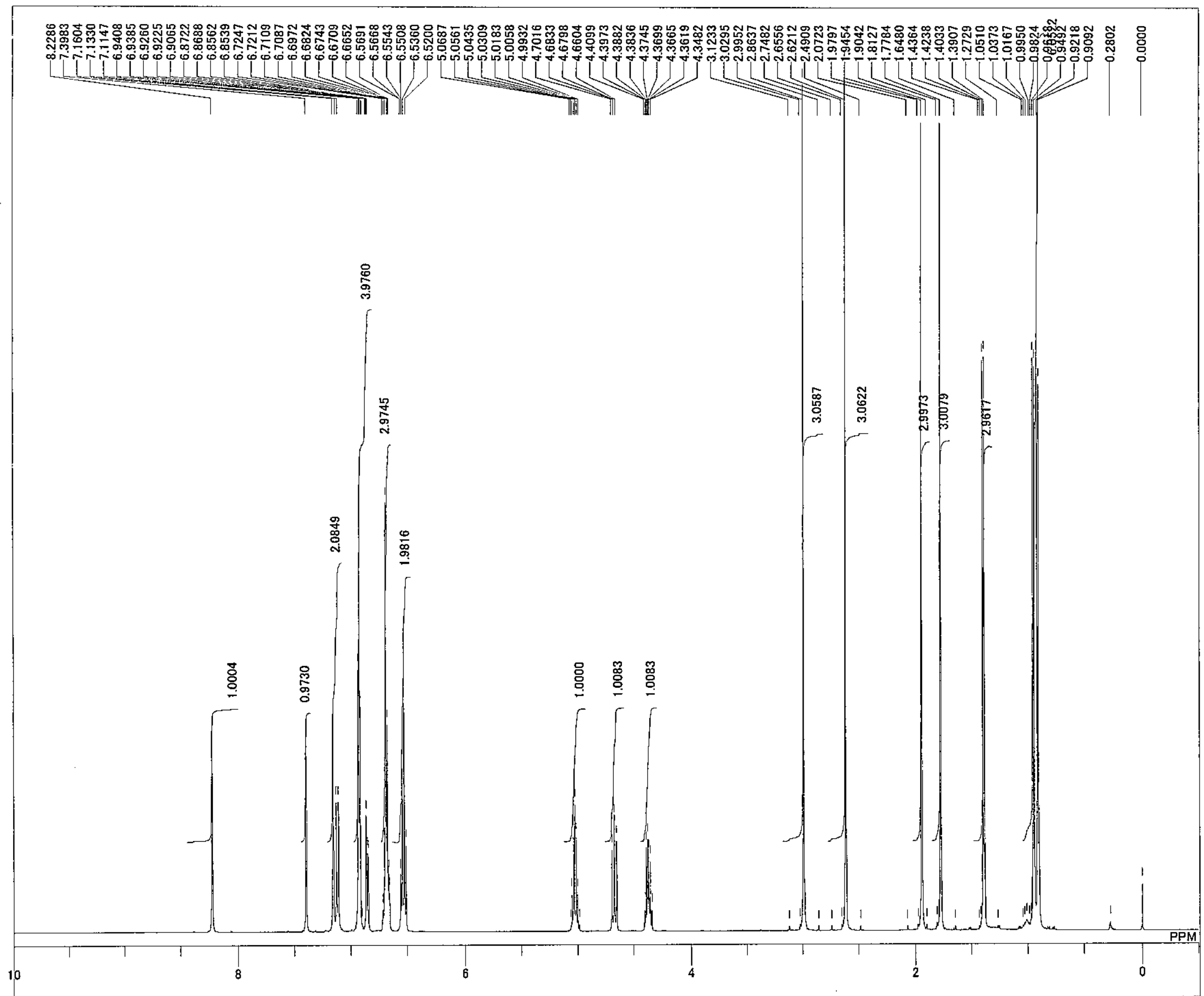

DFILE

COMNT

DATIM

OBNUC

OBFRQ

OBSET

POINT

FREQU

ACQTM

PW1

IRNUC

SLNNT

EXREF

RGAIN

data florian¥fb 436 prana -1 jdf

8-08-2007 20:20:50

H NMR.ex2 2

$495.13 \mathrm{MHz}$
$4.38 \mathrm{KHz}$

$4.38 \mathrm{KHz}$
$9.64 \mathrm{~Hz}$

$9286.78 \mathrm{~Hz}$
8

$1.7642 \mathrm{sec}$

$5.0000 \mathrm{sec}$

0.50 usec

C6D6 27.8

$0.12 \mathrm{ppm}$
30

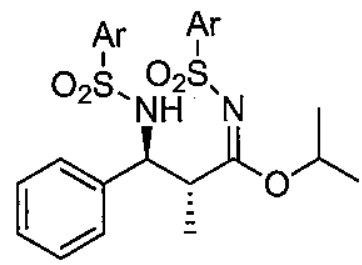

$\mathrm{Ar}=2,5-\mathrm{Xy} \mid \mathrm{yl}$

anti-7z 


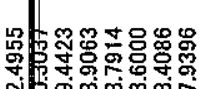

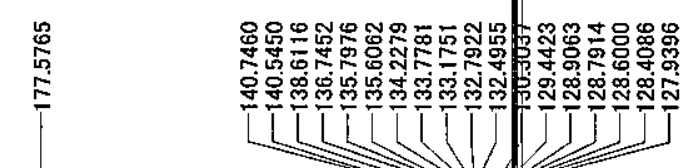

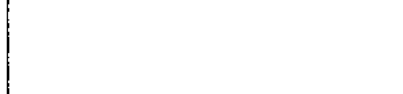$$
1
$$

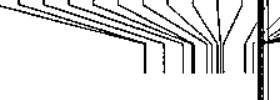

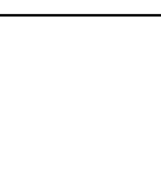
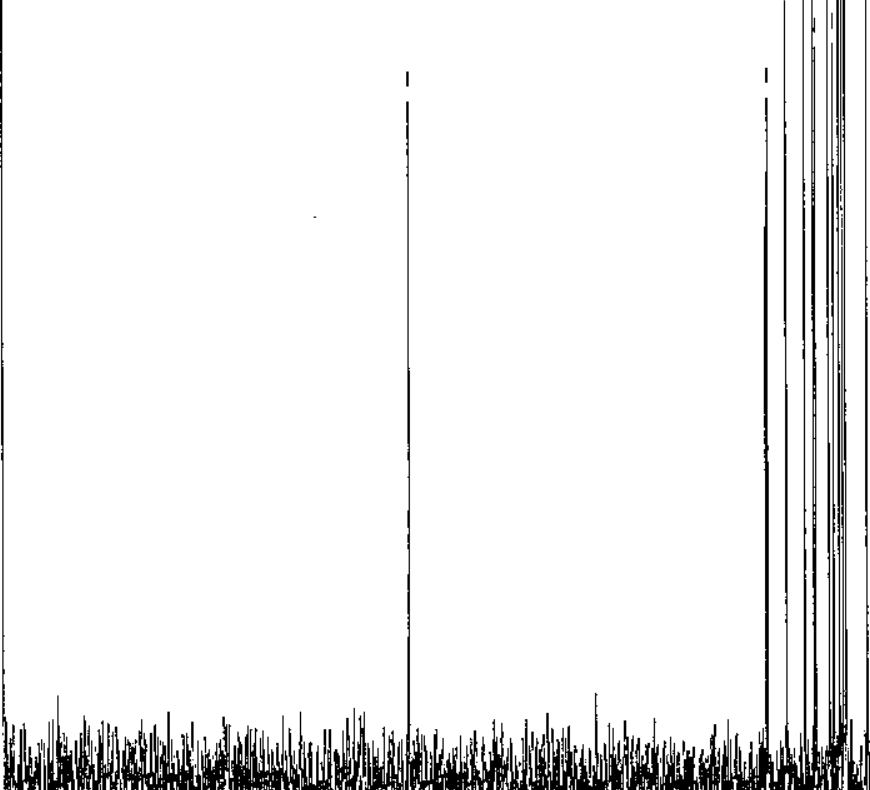
3.4.

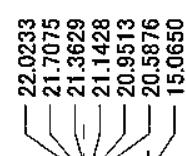

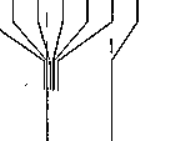


C::Zocuments and Settings¥ALCE2¥デスタトップ¥fb426remade_PROTON.3

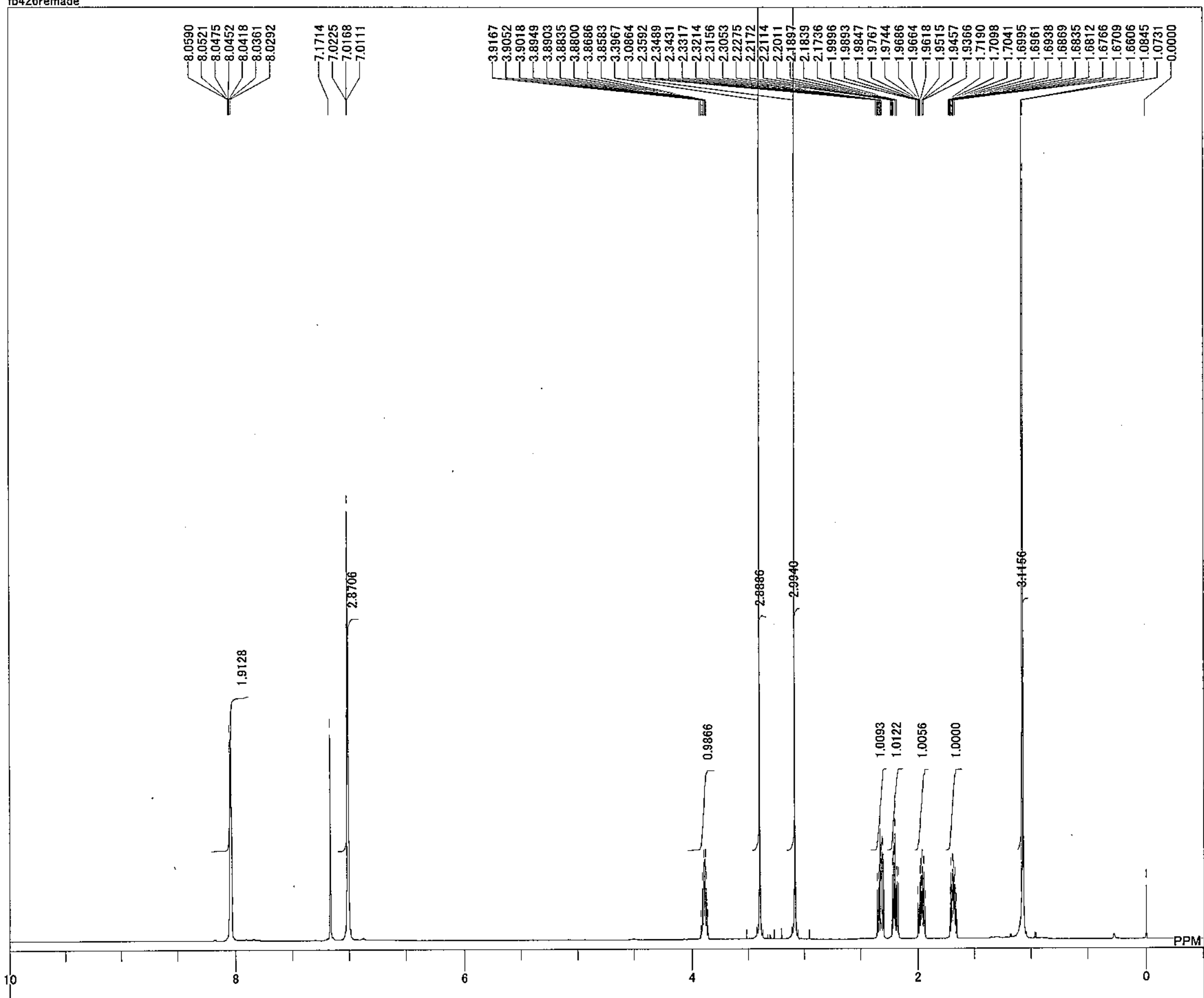

DFILE C:*Documents and Settings¥ALICE2¥デスク

DATIM

DATIM

EXMOD

OBFRQ

OBSET

POINT

FREQU

ACQTM

PD

PWNUC

CTEMP

SLVNT
EXREF
BF

RGAIN

作

single_pulse.ex2

$600.17 \mathrm{MHz}$
$5.30 \mathrm{KHz}$

$5.47 \mathrm{~Hz}$

20480
$14076.79 \mathrm{~Hz}$

$1.4549 \mathrm{sec}$

$4.0000 \mathrm{sec}$

$1 \mathrm{H}$

$\mathrm{C}^{2} \mathrm{D} 6{ }^{29.5 \mathrm{c}}$

$0.00 \mathrm{ppm}$
$0.12 \mathrm{~Hz}$
36

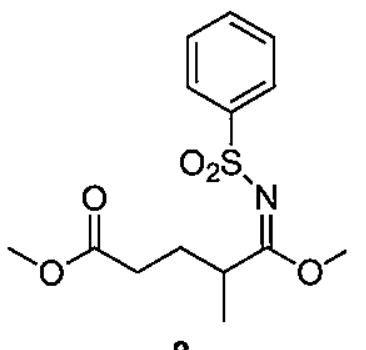

8 


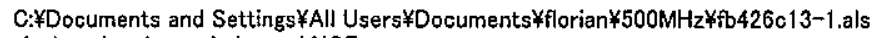
single pulse decoupled gated NOE
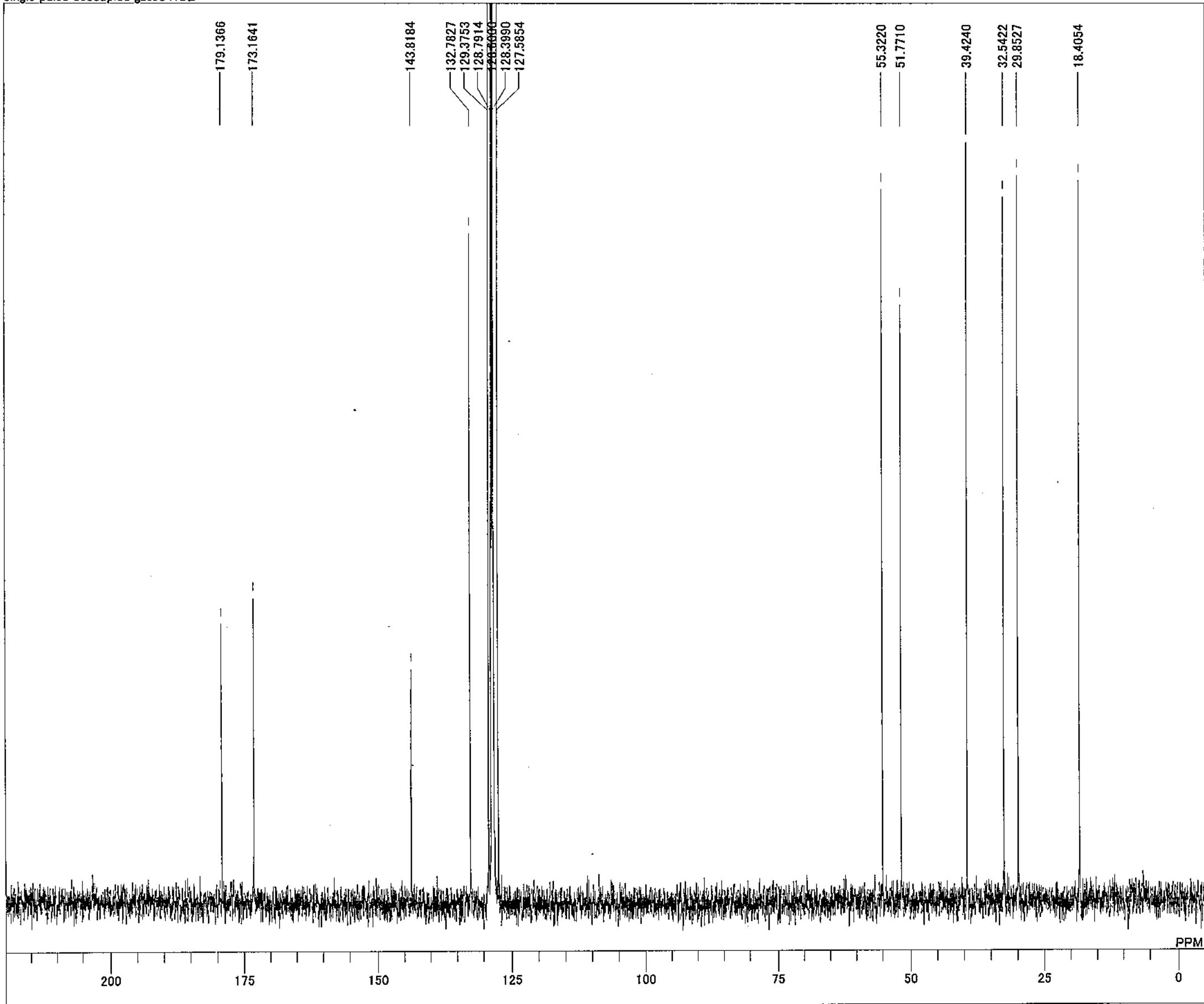

DFHE

single pulse decoupled gated NOE

-07-2007 16:06:25

13C NMR.ex2

OBFRQ $\quad 124.51 \mathrm{MHz}$

OBFIN

$\quad 6.00 \mathrm{~Hz}$

$\quad 31249.52 \mathrm{~Hz}$

SCANS

ACQTM

PW1

IRNUC

CTEMP

SLVNT

BX

RGAIN

C6D6 $25.4 \mathrm{c}$

$128.60 \mathrm{ppm}$

$1.20 \mathrm{~Hz}$

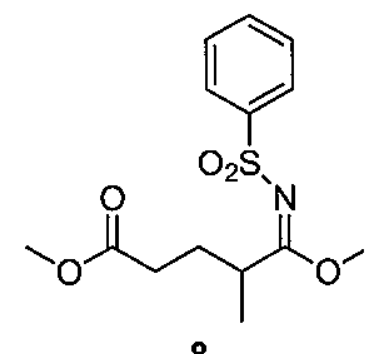

8 
DFILE C:\%Documents and Settings $¥ A l f$ Users\%Docu

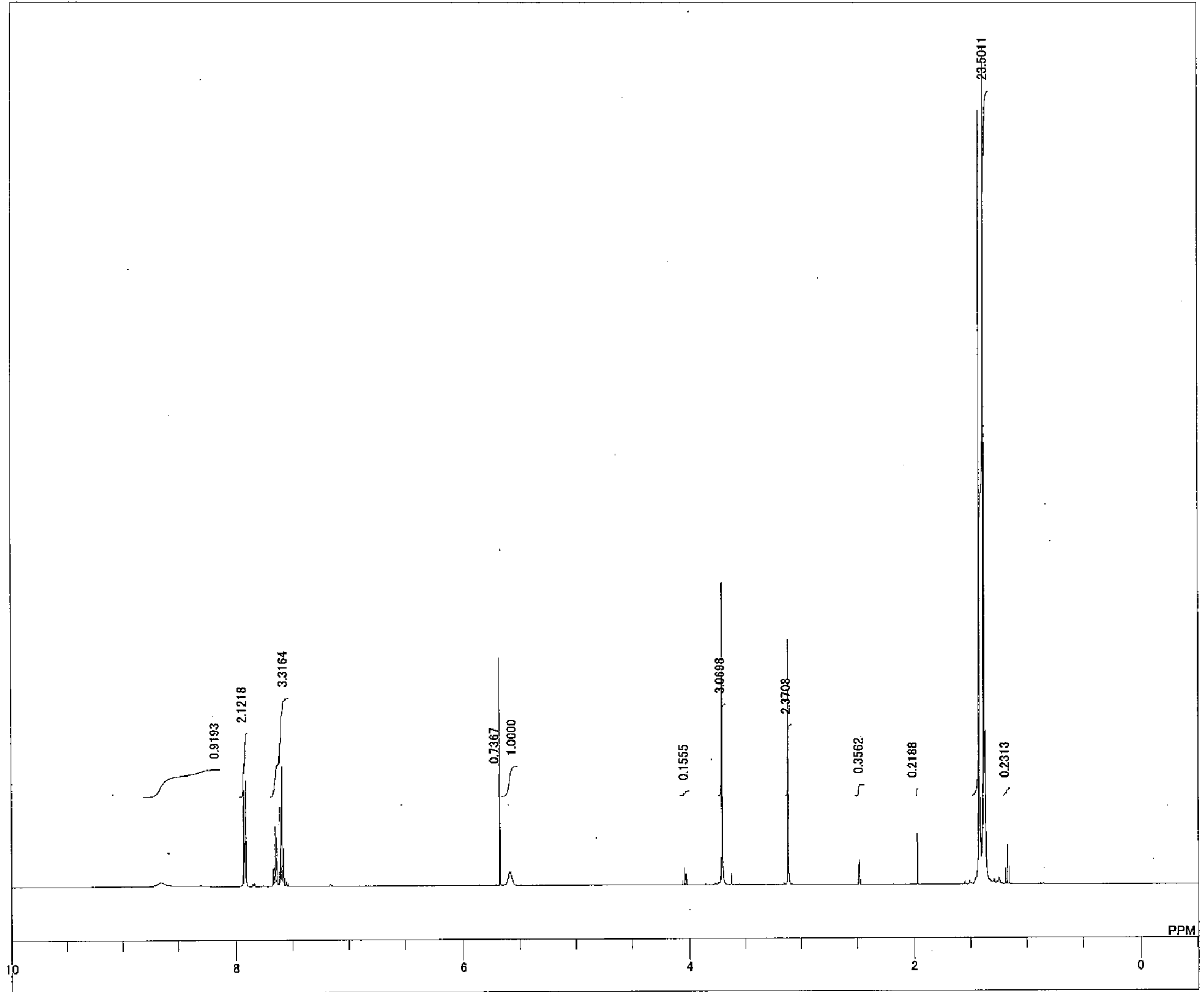

DATM 2707-data $11-08-2007$ 18:51:58

OBNUC

$1 \mathrm{H}$ NMR,ex2

OBSET $\quad 4.38 \mathrm{KHz}$

$\quad 9.64 \mathrm{~Hz}$

FREQU $\quad 7429.31 \mathrm{~Hz}$

SCANS

IRNUC $1 \mathrm{H} \quad 6.50 \mathrm{Us}$

CTEMP $70.0 \mathrm{c}$

$\begin{array}{ll}\text { SLVNT } & \text { DMSO } \\ \text { EXREF } & 2.49 \mathrm{ppm}\end{array}$

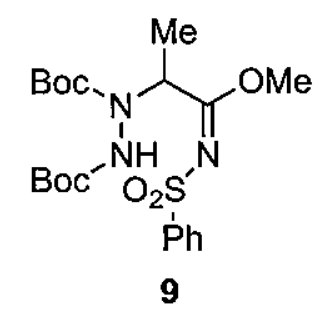

ACQTM $\quad 1.7642 \mathrm{sec}$

$\quad 5.0000 \mathrm{sec}$

BF $\quad 0.12 \mathrm{~Hz}$ 
C:FDocuments and Settings¥All Users¥Documents¥Matsubara $¥ 2701-2800 ¥ 2707-$ data_C-1.als 2707-data_c
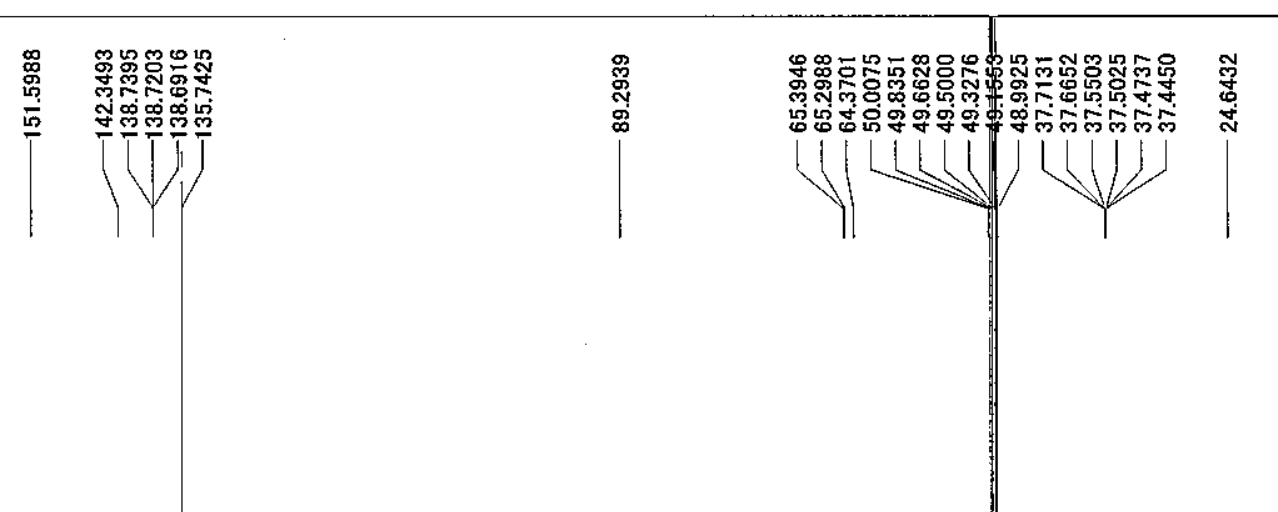

DFILE $\quad$ C:*Documents and Settings $* A l l$ Users*Docu

COMNT

OBNUC

EXMOD

OBFRQ

OBSE

$-08-2007$ 19:39:58

$13 \mathrm{C}$ NMR.ex2

$124.51 \mathrm{MHz}$

$3.45 \mathrm{KHz}$

FREQU $31249.52 \mathrm{~Hz}$

$\begin{array}{ll}\text { SCANS } & 1000 \\ \text { ACQTM } & 0.8389 \mathrm{seO}\end{array}$

$\quad 2.0000 \mathrm{sec}$

PRNUC $1 \mathrm{H}$.67 usec

CTEMP $70.0 \mathrm{c}$

SLVNT DMSO

BF

$49.50 \mathrm{ppm}$
$0.12 \mathrm{~Hz}$
60

(1)

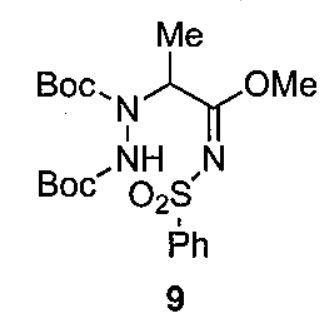

7.M. PPM

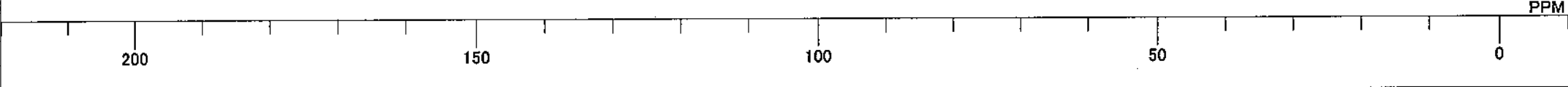


DFILE

COMN

DATIM

EXMOD

OBFRQ

OBSET

POINT

FREQU

SCANS

ACQTM

ACQTM

IRNUC

CTEMP
SLVNT
EXREF
BF

EXREF

RGAIN

Documents anơ Settings*All Users¥Docu

IH NMR.ex2

$495.13 \mathrm{MHz}$
$4.38 \mathrm{KHz}$

$7429.31 \mathrm{~Hz}$

8
$1.7642 \mathrm{sec}$

$5.0000 \mathrm{sec}$

$1 \mathrm{H} \quad 6.50 \mathrm{usec}$

DMSO $^{29.3 \mathrm{c}}$

$3.30 \mathrm{pprn}$
$0.12 \mathrm{~Hz}$

34

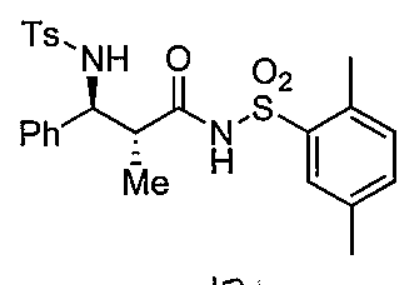

10

PPM 


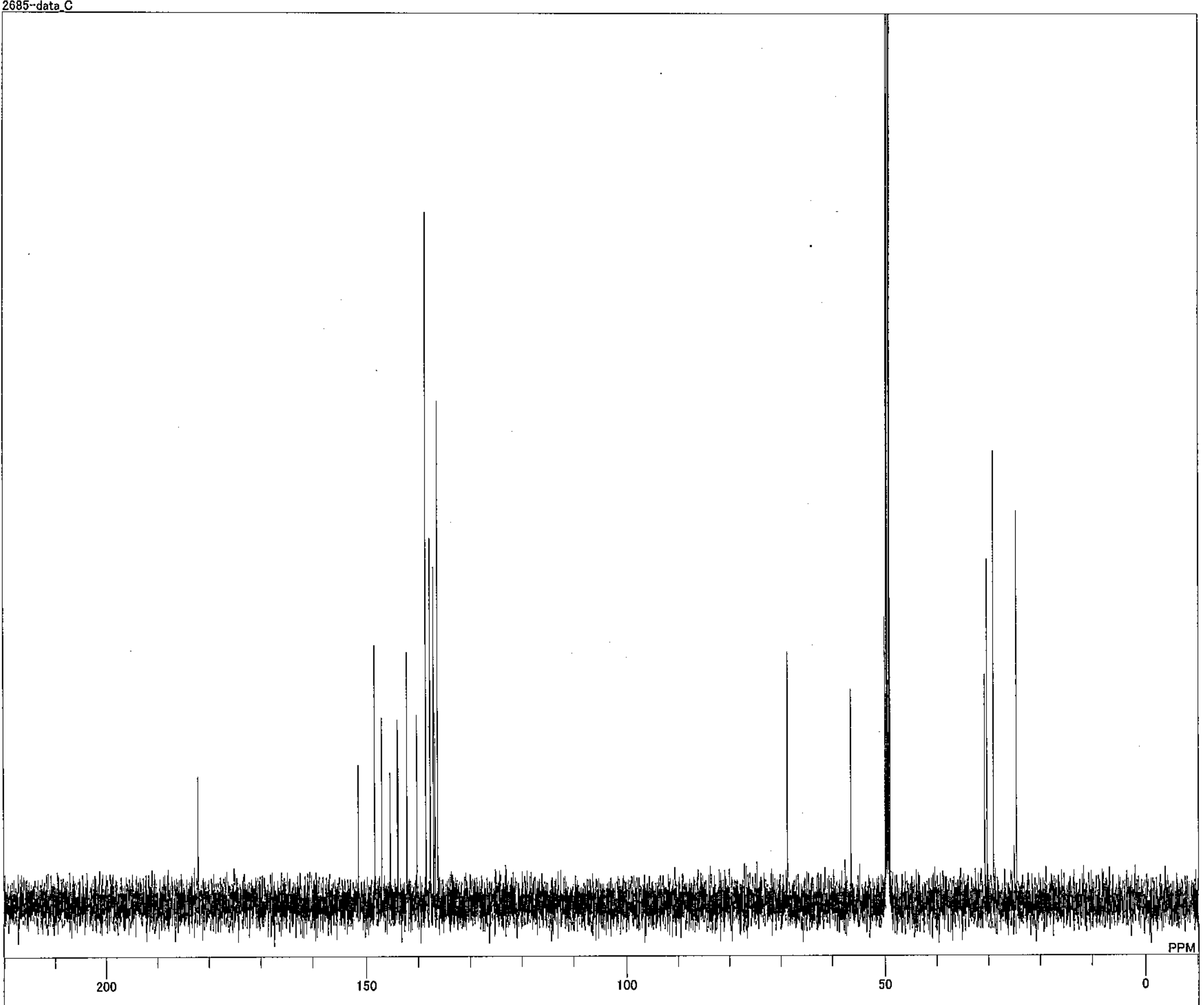

C:FDocuments and SettingsłAll Users*Docu

COMNT 2685-data C $18: 4: 6$

EXMOD

OBSET

13C

$13 \mathrm{C}$ NMR.ex2

$124.51 \mathrm{MHz}$
$3.45 \mathrm{KHz}$

26214

SCANS $\quad 124$

ACQTM $\quad 0.8389 \mathrm{sec}$

$\begin{array}{lr}\mathrm{PD} & 2.0000 \mathrm{sec} \\ \mathrm{PW} 1 & 3.67 \mathrm{usec}\end{array}$

IRNUC tH 3.67 use

CTEMP 29.7

$\begin{array}{ll}\text { EXREF } & 49.50 \mathrm{ppm}\end{array}$

BF

$49.50 \mathrm{ppm}$
$0.12 \mathrm{~Hz}$
56

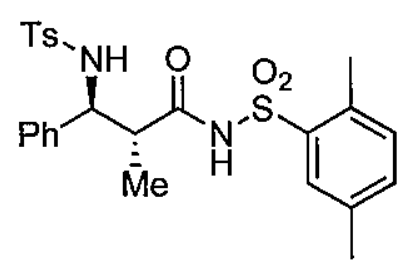

10 
C:\#Documents and Settings $¥ A$ All Users $¥ D$ ocuments $¥$ Matsubara $¥ 2701-2800 ¥ 2723$ lowconcentration-1.als

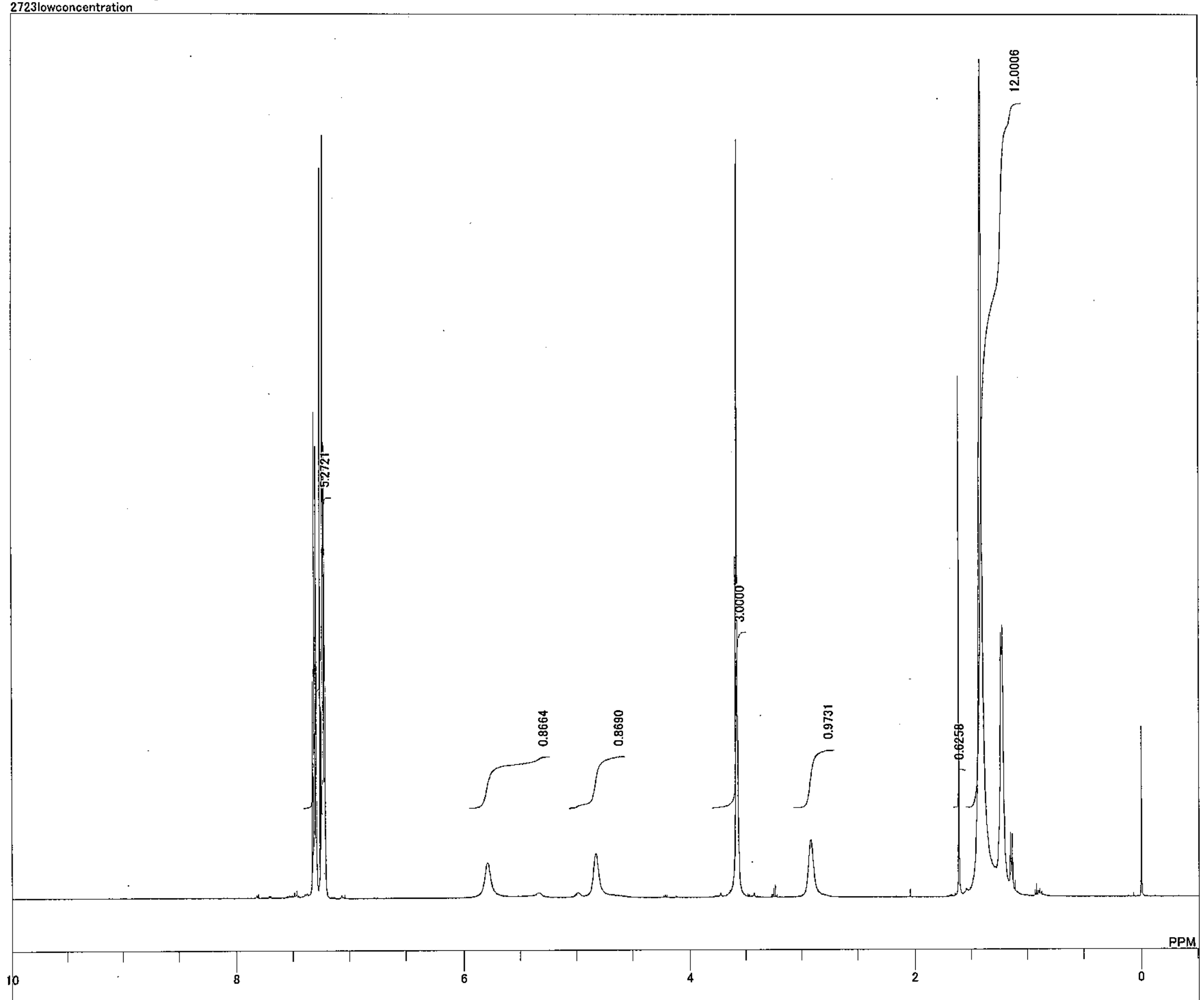

DFILE $\quad$ C:FDocuments and Settings $¥ A$ All Users $¥$ Docu

$\begin{array}{ll}\text { COMNT } & \text { 2723lowconcentration } \\ \text { DATIM } & 06-08-200721: 19: 23\end{array}$

EXMOD 1H NMR.ex2

$\begin{array}{lc}\text { OBFRO } & 495.13 \mathrm{MHz} \\ \text { OBSET } & 4.38 \mathrm{KHz}\end{array}$

$9.64 \mathrm{~Hz}$

13107

$7429.31 \mathrm{~Hz}$

ACQTM $\quad 1.7642 \mathrm{seO}$

$\quad 5.0000$ seo

6.50 usec

CTEMP
SLVNT 28.10
EXREF

$\begin{array}{ll}\text { EXREF } & 0.00 \mathrm{ppm} \\ \text { BF } & 0.12 \mathrm{~Hz}\end{array}$

RGAIN

$0.12 \mathrm{~Hz}$

Boc

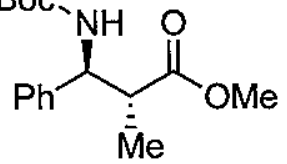

1.1 
C:¥Documents and Settings $¥ A l l$ Users $¥$ Documents $¥$ Matsubara $¥ 2701-2800 ¥ 2723-C$.als

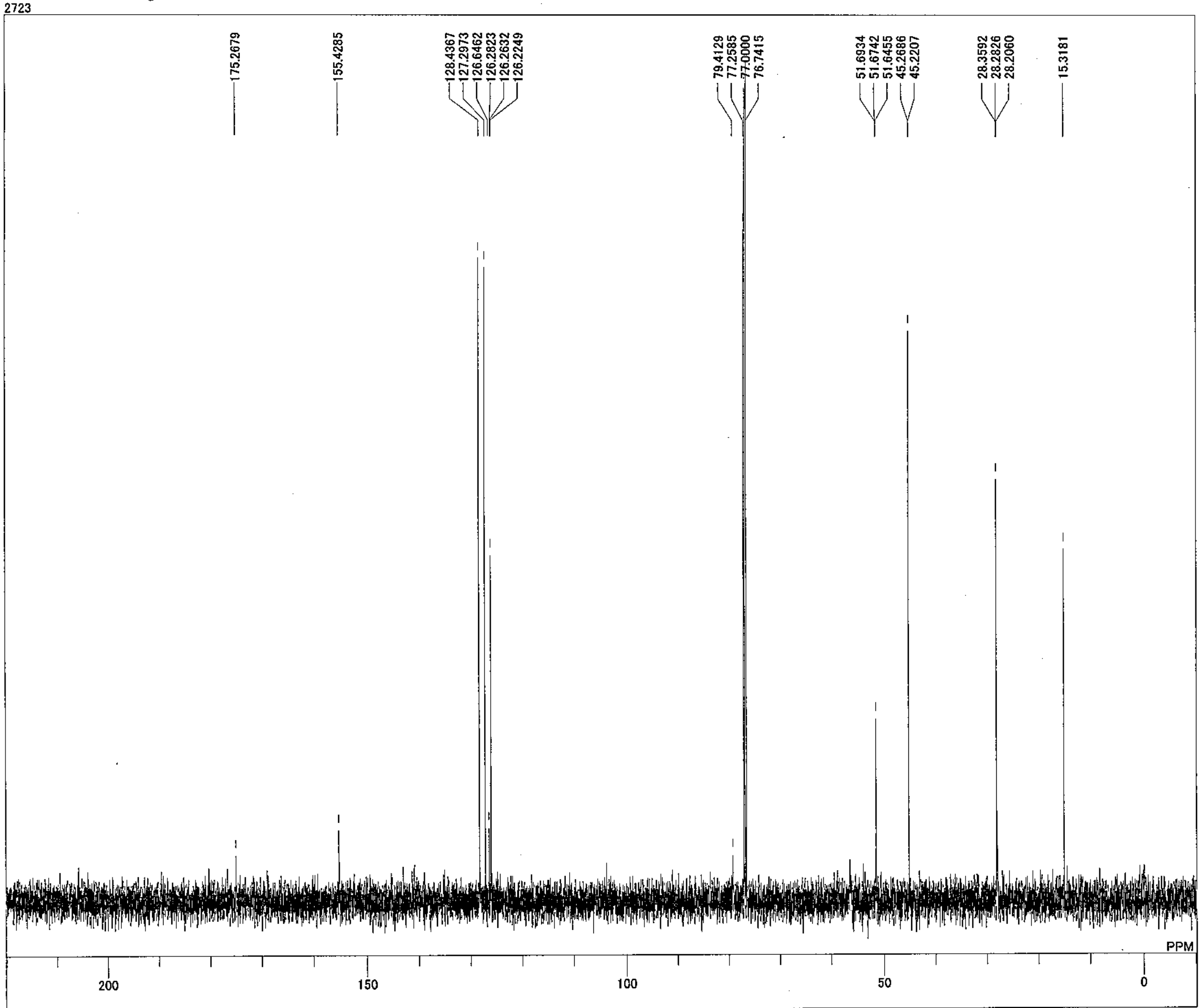

C:\&Documents and SettingsłAli Users¥Docu

DATIM $06-08-2007$ 13:24:51

$13 \mathrm{C}-2007132451$

EXMOD $13 \mathrm{C}$ NMR.eX2

OBSET $\quad 3.45 \mathrm{KHz}$ $6.00 \mathrm{~Hz}$ 26214

SCANS $\quad 88$

ACQTM $\quad 0.8389 \mathrm{sec}$

\begin{tabular}{ll} 
PW1 & $2.0000 \mathrm{sec}$ \\
\hline & $3.67 \mathrm{usec}$
\end{tabular}

CTEMP
SLVNT
EXREF

$\begin{array}{ll}\text { EXREF } & 77.00 \mathrm{ppm} \\ \text { BF } & 0.12 \mathrm{~Hz}\end{array}$

Boc

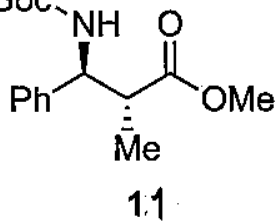




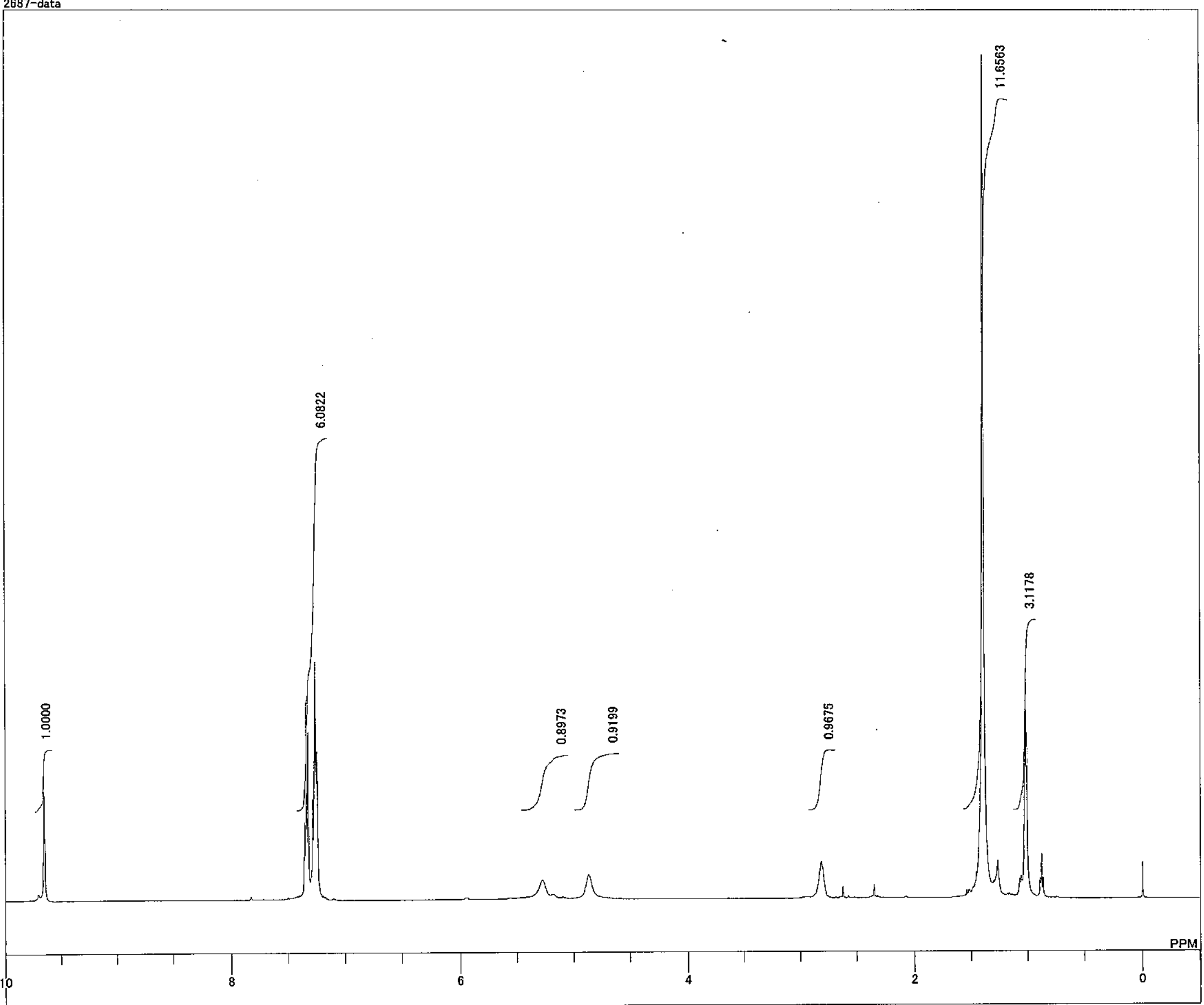

DFILE

Comin

OBNUC

EXMOD

OBSET

OBFIN

FREOU

SCAEQU

ACQTM

PD

PW1 1

IRNUC $\quad$ IH 29.0

SLVNT CDCL3 390

EXREF

BF

$0.00 \mathrm{ppm}$
$1.20 \mathrm{~Hz}$
32

Boc

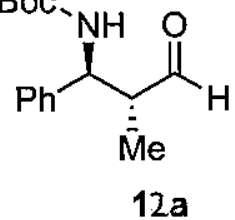


C: $: D o c u m e n t s$ and Settings $¥ A l l$ Users $¥$ Documents $¥$ Matsubara $¥ 2601-2700 \div 2687-$ data-13C-1.als

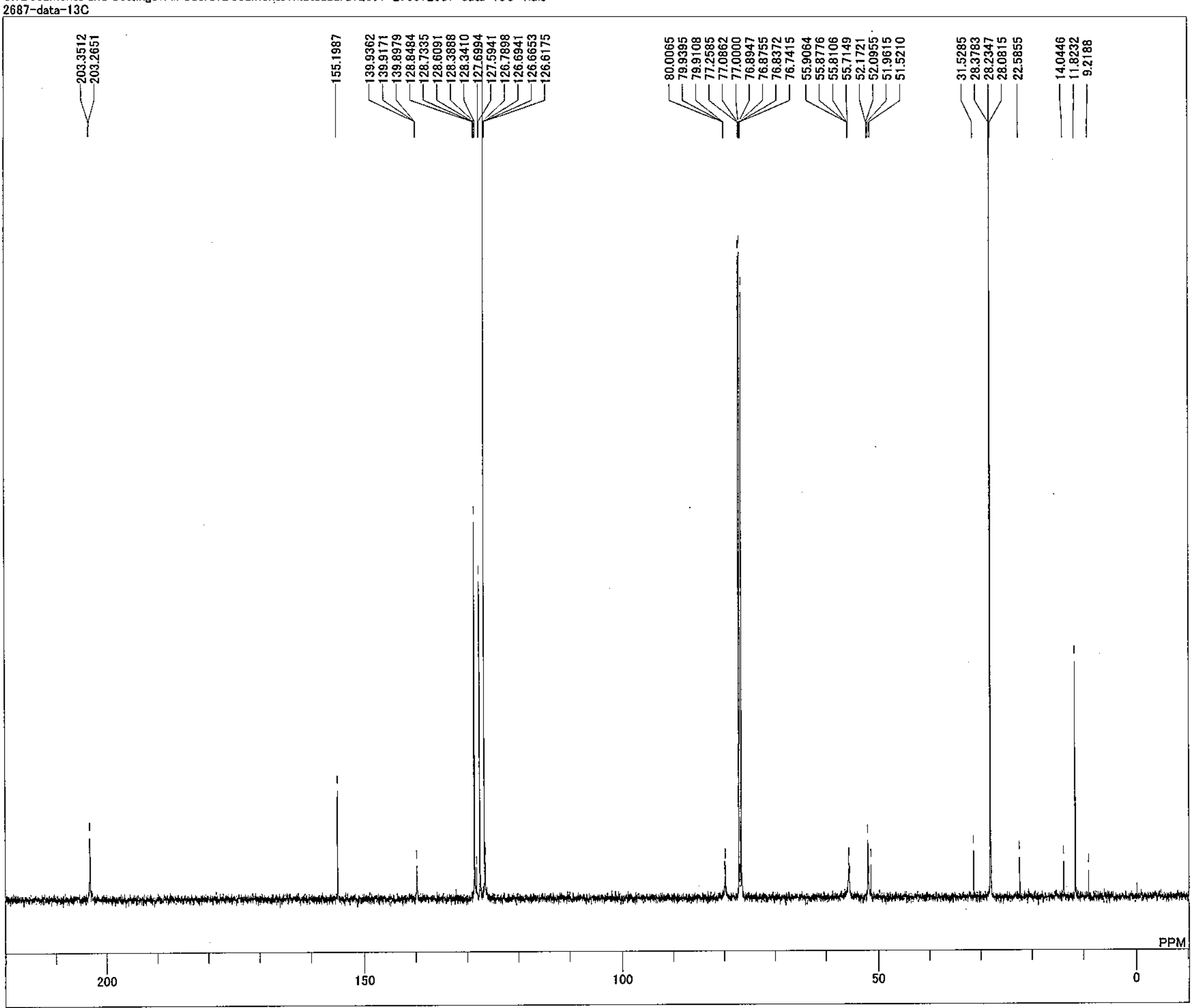

C:FDocuments and Settings:All Users¥Docu

COMN

OBNUC

EXMOD

OBFRQ

OBFIN

FREOU

SCANS

ACQTM

PD

IRNUC

CTEMP

SLVNT
EXREF
BF

RGAIN

21-08-2007 15:16:32

13C NMR.ex2

$124.51 \mathrm{MHz}$
$3.45 \mathrm{KHz}$

$6.00 \mathrm{~Hz}$

$31249.52 \mathrm{~Hz}$

817

817
$0.8389 \mathrm{sec}$

$2.0000 \mathrm{sec}$

1H 3.07 usec

$\operatorname{CDCL}^{29.4}$

$77.00 \mathrm{ppm}$
$1.20 \mathrm{~Hz}$

Boc

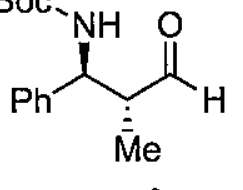

$12 \mathrm{a}$ 
C:¥Documents and Settings¥\#All Users $¥ D$ Documents $¥$ Matsubara $¥ 2601-2700 ¥ 2672-$ data-1.als

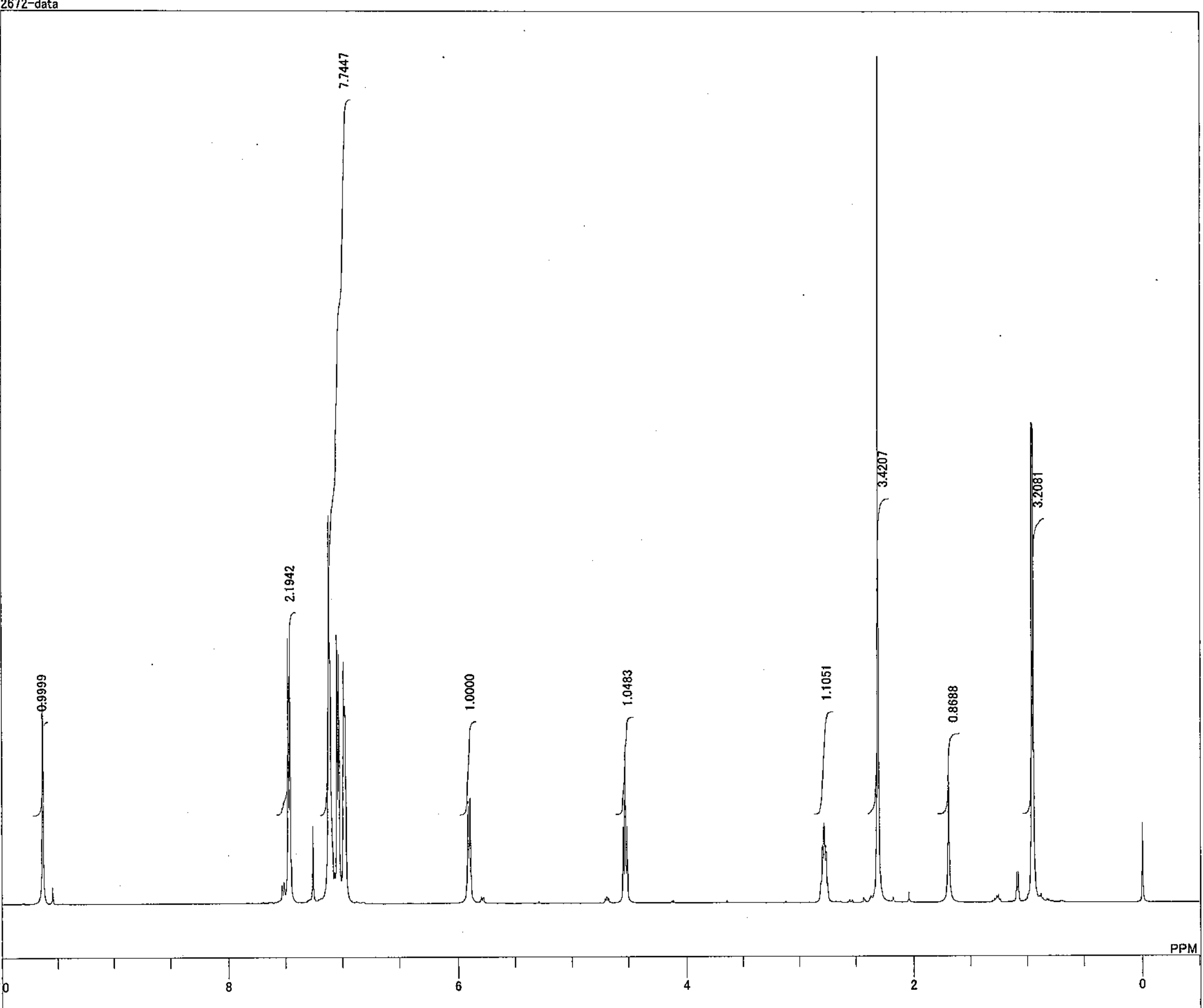

DFILE

COMNT

OBNUC

OBFRQ

OBFIN

FREQU

SCANS

ACQTM

PD

PW1
IRNUC
CTEMP

SLVNT

EXREF

RGAIN

Documents and Settings:All Users¥Docu C-data

iH NMR.ex2

$495.13 \mathrm{MHz}$
$4.38 \mathrm{KHz}$

$9.64 \mathrm{~Hz}$

$7429.31 \mathrm{H}$

8
$1.7642 \mathrm{sec}$

$5.0000 \mathrm{sec}$

$1 \mathrm{H}$

$\mathrm{CDCL}^{28.9}$

$0.00 \mathrm{ppm}$
$1.20 \mathrm{~Hz}$

$40 \mathrm{~Hz}$

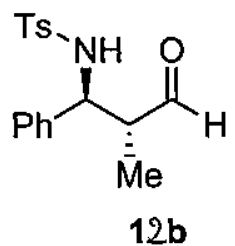


C:FDocuments and Settings $¥ A$ All Users $¥$ Documents $¥ M a t s u b a r a ¥ 2601-2700 ¥ 2672$ C -1 .als

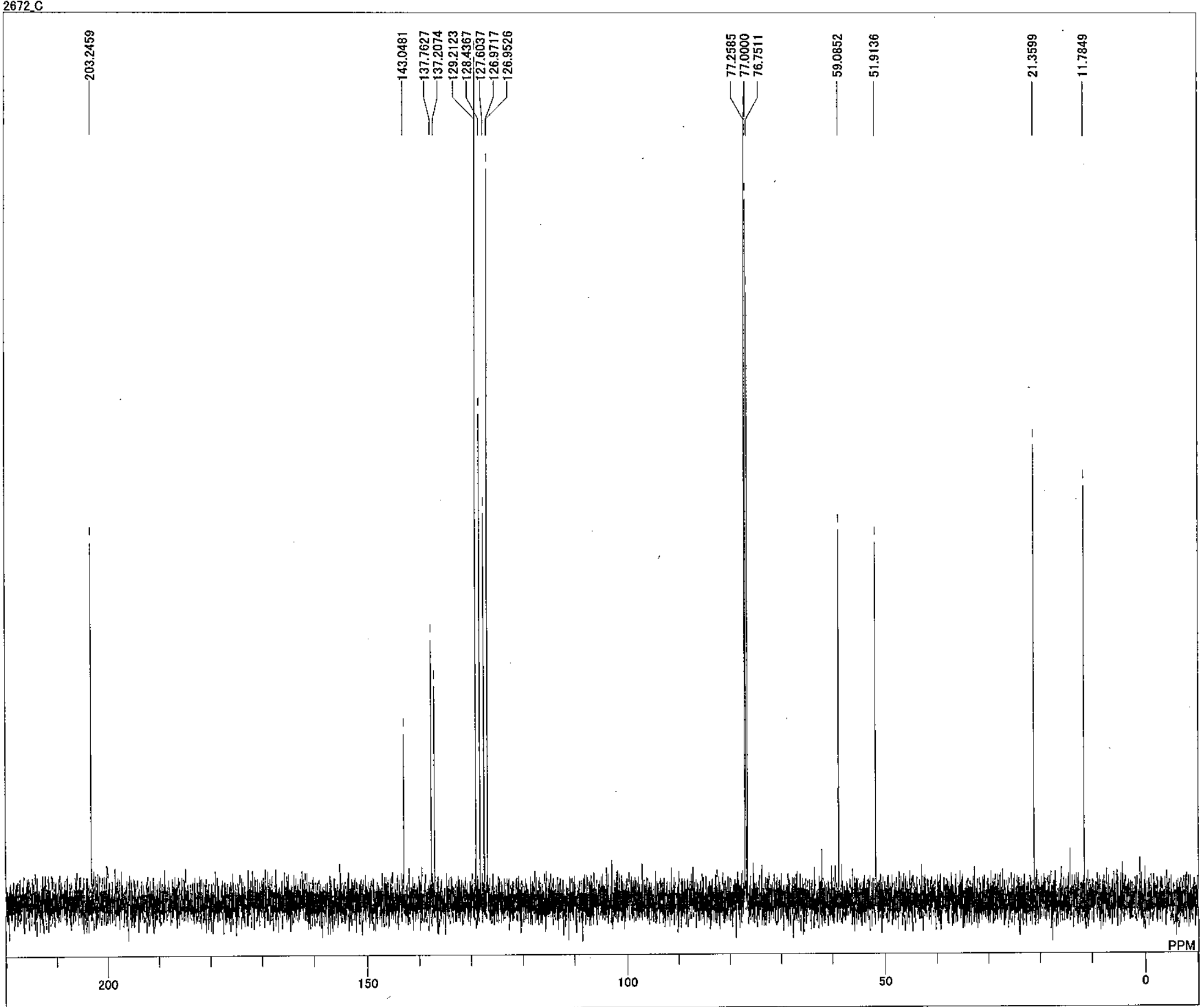

DFILE C:\#Documents and Settings $¥ A l l$ Users¥Docu

COMNT

DATIM

130 -2007 10.33:42

OBSET

POINT
FREQU

$\begin{array}{ll}2.0000 \mathrm{sec} \\ \text { PW1 } & 3.67 \text { usec }\end{array}$

IRNUC

SLVNT $\operatorname{CDCL} 3.90$

$\begin{array}{ll}\text { EXREF } & 77.00 \mathrm{ppm} \\ \text { BF } & 0.12 \mathrm{~Hz} \\ \text { RGAIN } & 50\end{array}$

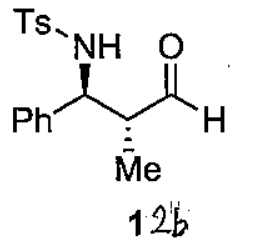

$12 b$ 


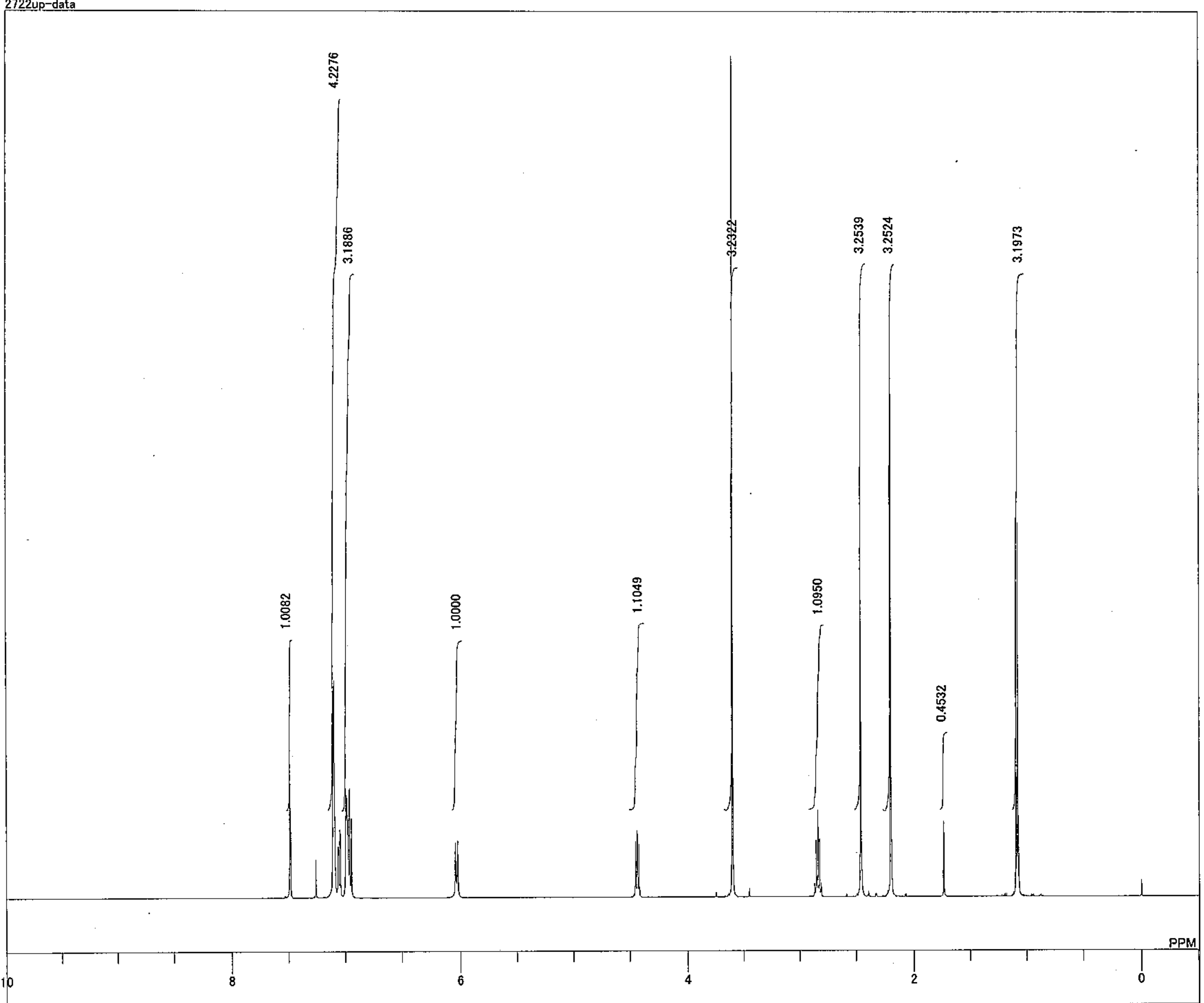

DFILE C:\#Documents and Settings $¥ A l l$ Users $¥ D o c u$

COMNT

DATIM

OBNUC

OBFRQ

OBSET

PBINT

POINT

SCANS

ACQTM

PD

PWN

$29.6 \mathrm{c}$

$\begin{array}{ll}\text { EXREF } & 0.00 \mathrm{ppm} \\ \text { BF } & 0.12 \mathrm{~Hz}\end{array}$

RGAIN

22up-data $13 \cdot 29 \cdot 50$

H

$495.13 \mathrm{MHz}$
$4.38 \mathrm{KHz}$

$9.64 \mathrm{~Hz}$

$7429.31 \mathrm{~Hz}$

$1.7642 \mathrm{sec}$

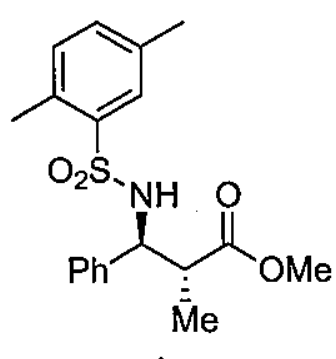

13 
C:FDocuments and Settings $¥ A l l$ Users¥Documents $¥ M a t s u b a r a ¥ 2701-2800 * 2722$ up-data_C-1.als

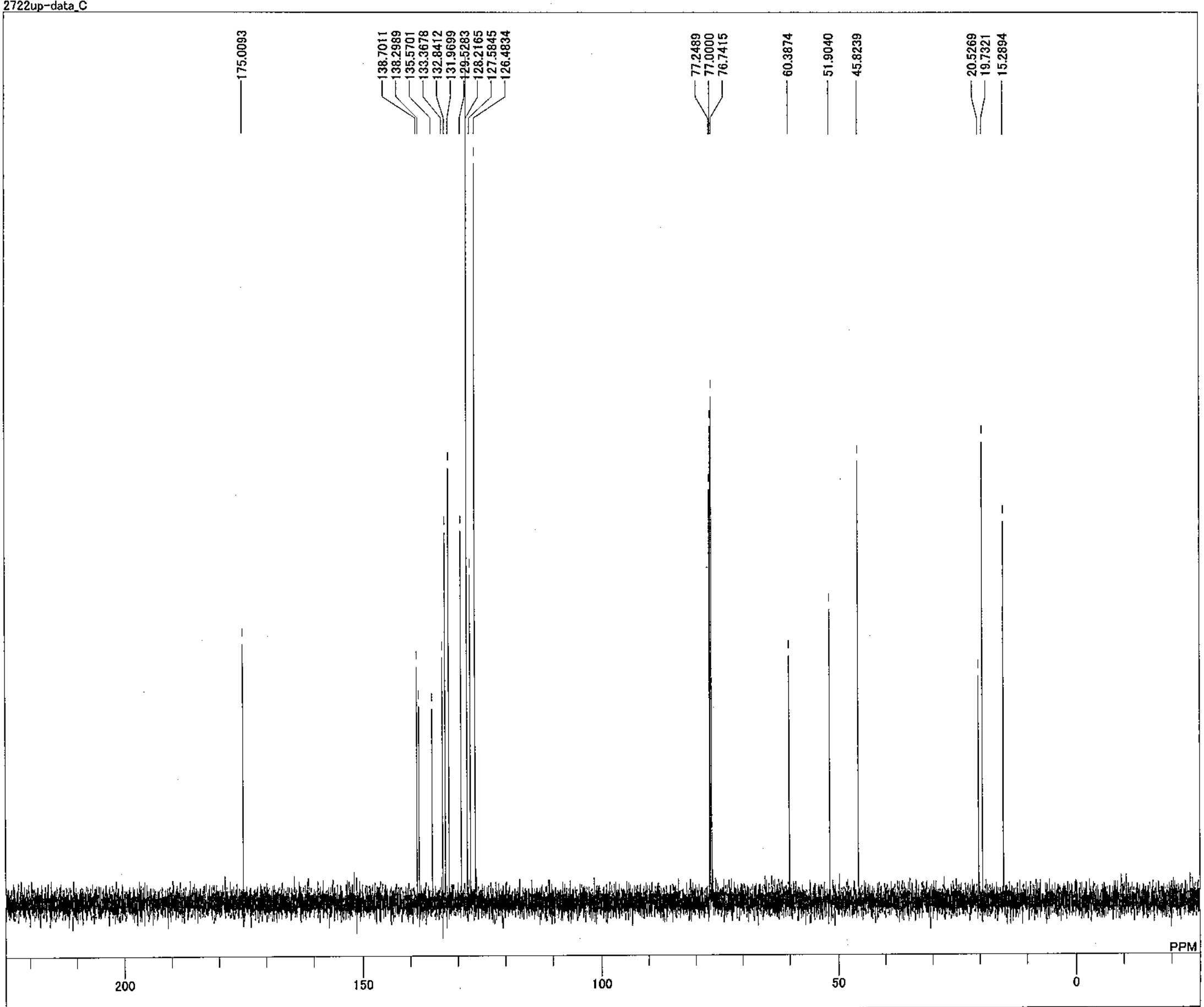

DFILE C:Focuments and Settings:Ali Users¥Docu

DATIM 11-08-2007 13:33:34

DATIS

EXMOD - 13C NMR EX2

$\begin{array}{lc}\text { OBFRQ } & 124.51 \mathrm{MHz} \\ \text { OBSET } & 3.45 \mathrm{KHz}\end{array}$

$6.00 \mathrm{~Hz}$

FREQU $3124952 \mathrm{~Hz}$

$\begin{array}{lc}\text { SCANS } & 63 \\ \text { ACQTM } & 0.8389 \mathrm{sec}\end{array}$

$2.0000 \mathrm{sec}$

PW1 $1 \mathrm{H} 3.67$ use

CTEMP $29.8 \mathrm{c}$

$\begin{array}{ll}\text { EXREF } & 77.00 \mathrm{ppm} \\ \text { BF } & 0.12 \mathrm{~Hz} \\ \text { RGAIN } & 52\end{array}$

RGAIN

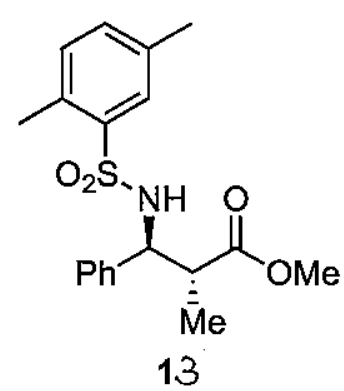

13 